\title{
Maine de BIRAN
}

\section{2 \\ II \\ Mémoire sur \\ la décomposition de la pensée}

\author{
Notes critiques \\ par \\ Pierre Tisserand
}

Un document produit en version numérique par Mme Marcelle Bergeron, bénévole Professeure à la retraite de l'École Dominique-Racine de Chicoutimi, Québec et collaboratrice bénévole

Courriel: mailto:mabergeron@videotron.ca

Site web: http://slsj.areq.qc.net/

Dans le cadre de la collection: "Les classiques des sciences sociales" dirigée et fondée par Jean-Marie Tremblay, professeur de sociologie au Cégep de Chicoutimi

Site web: http://www.uqac.ca/Classiques_des_sciences_sociales

Une collection développée en collaboration avec la Bibliothèque

Paul-Émile-Boulet de l'Université du Québec à Chicoutimi

Site web: http://bibliotheque.uqac.uquebec.ca/index.htm 
Un document produit en version numérique par Mme Marcelle Bergeron, bénévole, professeure à la retraite de l'École Dominique-Racine de Chicoutimi, Québec courriel: mailto:mabergeron@videotron.ca

site web : http://slsj.areq.qc.net - Secteur Chicoutimi-Valin

À partir de :

Marie François Pierre Gontier de Biran, dit Maine de Biran, (1766-1824), Mémoire sur la décomposition de la pensée, tome II et Notes critiques par Pierre Tisserand.

Une édition électronique réalisée à partir du texte publié en 1952: Mémoire sur la décomposition de la pensée, tome II, par les Presses Universitaires de France, 108, Boulevard St-Germain, Paris, 282 pp.

Polices de caractères utilisés :

Pour le texte: Times, 12 points.

Pour les citations : Times 10 points.

Pour les notes de bas de page : Times, 10 points.

Édition électronique réalisée avec le traitement de textes Microsoft Word 2001 pour Macintosh.

Mise en page sur papier format

LETTRE (US letter), 8.5' x 11'’)

Édition complétée le 28 juin 2004 à Chicoutimi, Québec. 


\section{Maine De Biran}

Mémoire sur la décomposition de la pensée

Tome II

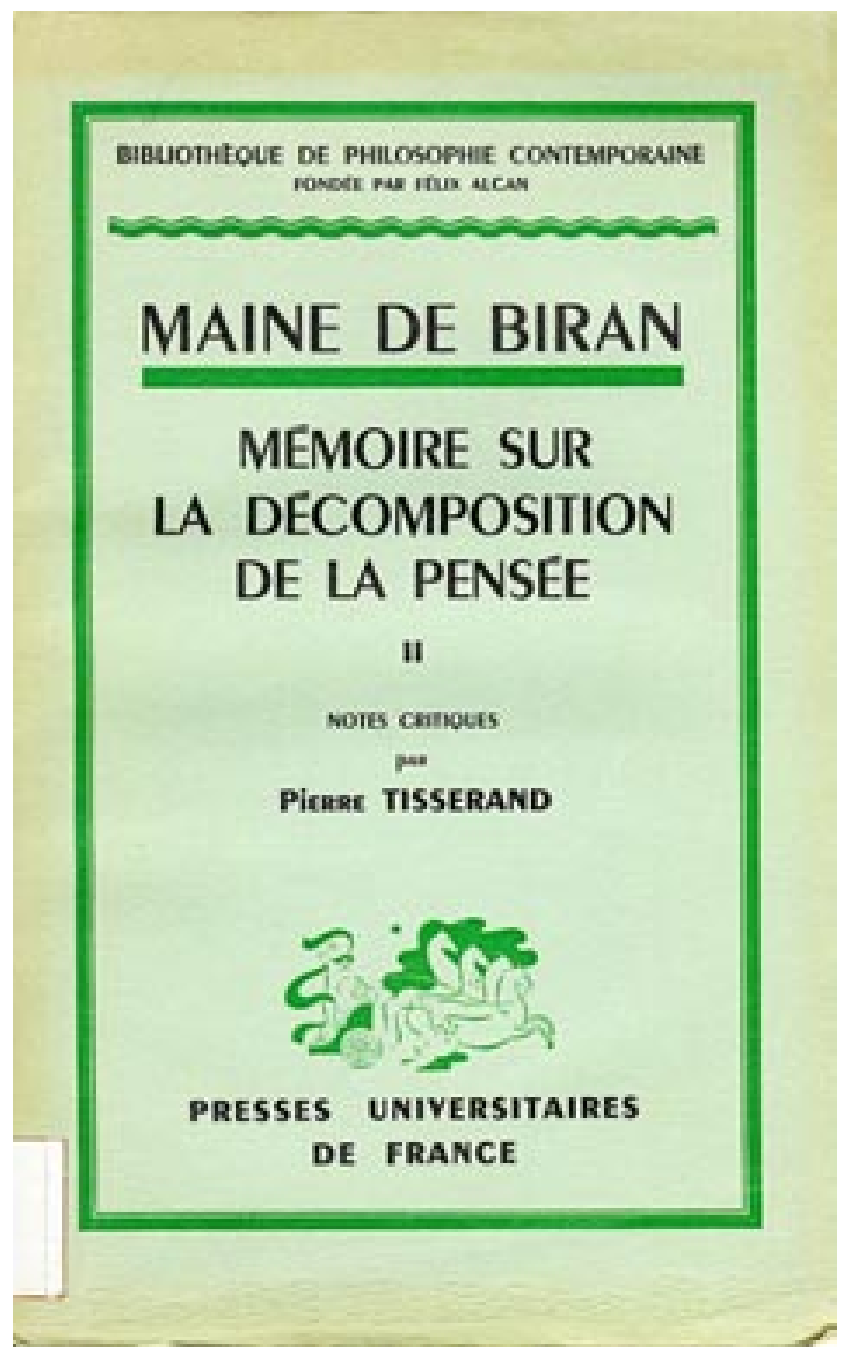

Paris : Presses Universitaires de France, 1952, 282 pp. 


\section{Table des matières}

Deuxième section. - D'une analyse des sens considérée sous le rapport d'origine et de dérivation de deux ordres de Facultés et d'idées élémentaires.

Chapitre I. - De l'association première de l'effort avec les affections simples. Comment celles-ci sont rapportées aux organes. - Fondement de différentes classes de sensations. Comment on pourrait dériver de l'exercice d'un sens, tel que l'odorat, diverses facultés et idées.

Chapitre II. - Continuation du précédent. Analyse et dérivation des facultés et des idées élémentaires qui peuvent se rapporter à l'exercice d'un sens particulier. Premier exemple pris de l'odorat.

Chapitre III. - Des fonctions de l'ouïe et de la voix. Des facultés et des idées élémentaires qui peuvent en être dérivées.

Chapitre IV. - Analyse de la vision. Des facultés originelles et des idées qui s'y rapportent.

Chapitre V. - Des fonctions du toucher. Analyse des facultés et des idées élémentaires qui s'y rapportent.

Résumé général. Tableau et projet d'une division et d'une énumération des facultés humaines.

Réflexions sur les résultats de la méthode de décomposition précédemment expliquée. Projet d'analyse des mêmes difficultés considéré dans un ordre supérieur d'exercice.

Troisième section. - D'une analyse des facultés humaines considérées dans leur exercice général. L'association commune des sens entre eux et de leurs produits composés à des signes artificiels.

Chapitre I. - Fondement de l'institution des signes. Second ordre de facultés intellectuelles. Parallélisme de cet ordre avec le premier tableau correspondant de leur division.

Chapitre II. - Ordre passif intellectuel. Première classe de composés de cet ordre 
Chapitre III. - Ordre actif intellectuel. Première classe de composés. (Voyez le tableau.)

Chapitre IV. - Ordre actif intellectuel. Deuxième classe de composés. (Voyez le tableau.)

Chapitre V.- Ordre actif intellectuel. Deuxième et troisième classes. (Voyez le tableau.)

Résumé général. 


\section{Deuxième section}

D'une analyse des sens considérée sous le rapport d'origine et de dérivation de deux ordres de facultés et d'idées élémentaires

\section{Chapitre I}

De l'association première de l'effort avec les affections simples. Comment celles-ci sont rapportées aux organes. - Fondement de différentes classes de sensations. Comment on pourrait dériver de l'exercice d'un sens, tel que l'odorat, diverses facultés et idées.

$\underline{\text { Retour à la table des matières }}$

Réduit au sentiment absolu de l'existence ou aux impressions purement affectives qui le constituent, l'être organisé vivant soutient bien, comme tel, des relations essentielles avec divers agents appropriés qui l'environnent. Il est même dirigé suivant de tels rapports, avec une assurance et une sorte d'infaillibilité, signes certains du physique des lois qui le régissent et l'entraînent dans le cercle assez uniforme d'une vie toute sensitive, presque sans perturbation, comme sans connaissance.

Les sphères roulantes suivent dans les espaces célestes, les lois invariables de l'attraction qu'elles ignorent; les molécules des corps, placées dans les mêmes circonstances, manifestent toujours les mêmes affinités électives. Les affinités organiques ou animales, plus compliquées, paraissent avoir, il est vrai, bien moins de constance et de fixité ; mais la fibre vivante, l'élément sensible, et les combinaisons qui s'en forment, n'en suivent pas leurs lois avec moins d'aveuglement et de nécessité. 
L'être intelligent seul connaît les résultats des rapports auxquels il est soumis par sa nature; lui-même se donne une direction et un but, se rend compte des moyens, les veut et en dispose.

Or, l'être intelligent n'est pas tel, uniquement, parce qu'il est susceptible, en vertu de son organisation, d'être affecté et de se mouvoir, mais parce qu'il est capable d'apercevoir les modifications qu'il éprouve comme sentant, et les actes qu'il détermine comme moteur. Or, le seul fait de la détermination de ses actes est essentiellement lié à l'aperception, tandis que la simple réceptivité sensitive des modes peut en être séparée : voilà le principe. Ces deux sortes de facultés commencent-elles ensemble, ont-elles même origine, mêmes conditions, mêmes instruments organiques ? Voilà ce qu'il fallait rechercher d'abord.

S'il était permis maintenant de rendre sensible, par quelque comparaison, la différence réelle qui peut exister entre la force vitale ou sensitive, qui détermine les actes automatiques de l'instinct, et celle qui dirige la locomotion et les actes de conscience, ne pourrait-on pas hasarder de dire que cette différence est comparable peut-être, jusqu'à un certain point, à celle qui existe entre les forces chimiques d'une part, qui, travaillant les éléments de la matière, transformant leurs composés les uns dans les autres, amènent tant de révolutions successives dans le sein ou sur la surface du globe, et celles de gravitation ou d'impulsion tangentielle d'autre part, dont les rapports déterminent la forme constante de l'orbite, et l'ordre régulier des mouvements planétaires?

La dynamique intellectuelle serait-elle plus fondée, que la dynamique des corps, à réduire des forces si diverses à une seule ? et l'observation intérieure ne motiverait-elle pas des distinctions aussi nécessaires que celles qui ressortent, en astronomie, de l'observation et du calcul ?

Poursuivons l'analyse de ces forces distinctes et non séparées, qui animent le microcosme, dans celles de leurs produits mixtes et composés, puisque c'est sous cette forme seule que les forces mêmes rentrent dans le champ propre et naturel de notre observation.

I. - Dans le déploiement constant et répété de la même force motrice, directement irradiée d'un centre unique, et le mode d'effort, seul primitivement relatif, qui lui correspond, se trouve constitué le sujet, par rapport au terme organique inerte, qui résiste à son action. Lorsque ce terme multiple a été circonscrit dans ses parties comme immédiatement soumises à la même force, alors seulement, les impressions reçues par ces dernières, en vertu de leur affectibilité propre, deviennent particulières et relatives de générales et absolues qu'elles pouvaient être dans l'origine ; alors aussi seulement, elles ont le caractère de véritables sensations composées du premier ordre, où entre un 
élément individuel constant, savoir la forme une de l'aperception ou du jugement, suivant laquelle les impressions sont rapportées à un siège organique déterminé.

Le premier acte, par lequel les impressions, sensibles sont rapportées aux organes qu'ils affectent, n'a pu échapper, malgré toute l'intimité des habitudes, aux esprits profondément réfléchis, qui ont tenté d'analyser l'intelligence humaine, jusque dans ses éléments constitutifs. Mais dès qu'ils l'attribuaient à quelque vertu innée, ou le considéraient comme une forme inhérente à la sensibilité, inséparable absolument de son exercice, ils devaient bien l'admettre sans conditions, ou même repousser l'idée qu'il fût possible d'assigner quelque condition particulière, sur laquelle, un tel acte peut originairement se fonder. Une observation assez simple et péremptoire, ce me semble, leur échappait; c'est, que si ce rapport primitif des affections aux parties, qui les souffrent, était aussi naturel, aussi nécessaire et invariable qu'ils le supposaient, et qu'il devrait l'être, en effet, s'il était inhérent à la sensibilité, ils n'eussent jamais pu l'observer ni le noter, quelle que fût leur sagacité ou leur finesse de tact dans ces matières.

«Ce raisonnement confus, ou ce jugement naturel, dit Malebranche, qui applique au corps ce que l'âme sent, n'est qu'une sensation qu'on peut dire composée. \ On trouve ici la profondeur d'une première analyse qui devait, ce semble, en amener beaucoup d'autres car puisqu'il y a un composé, il y a donc aussi des éléments simples, ou des affections qui ne sont pas encore des sensations, comme des jugements qui n'en sont pas non plus, Mais toute analyse ultérieure se trouve bien arrêtée par le point, de vue qui considère ces jugements comme naturels (ce qui signifie ici innés), et qui, d'une autre part, les taxe d'illusions ou d'erreurs : chose pourtant assez difficile à concilier; car la démonstration de l'erreur suppose la preuve d'une vérité contraire ${ }^{1}$. Il faut au moins deux chances réelles ou intelligibles; or quelle est celle où l'âme, le moi apercevrait, jugerait que c'est lui qui souffre une douleur physique, sans rapporter cette impression à quelque partie du corps ?

Locke, partant des idées de sensation, et les prenant toutes faites, telles qu'il les trouvait dans notre expérience, ne paraît pas douter que la sensation ait pu jamais exister autrement que sous la forme d'idée. Il était si loin de soupçonner un composé dans ce premier ordre de sensations, que l'école cartésienne avait pourtant signalé comme tel, qu'il ne le reconnût même pas dans les perceptions qui représentent les objets hors de nous.

Mais lorsque Condillac eut conçu le projet de remonter jusqu'à l'origine de toute idée et d'anatomiser, pour ainsi dire, les différentes espèces de sensations, pour assigner la part contributive de chacune d'elles dans l'entendement

1 Recherche de la Vérité, liv. I, chap. I. 
humain, qui est censé en être le résultat, ce philosophe, se plaçant très près du point où tout commence, dut reconnaître d'abord que les affections premières et simples de la sensibilité, n'ayant dans leur caractère intrinsèque rien qui portât, pour ainsi dire, le cachet de l'objet qui les occasionne, ni du siège qu'elles occupent, cette connaissance ou ce jugement qui se trouve en effet uni actuellement avec toutes nos sensations, devait avoir dans quelqu'une d'elles un fondement ou un mobile particulier. (C'est ainsi que le point de vue cartésien put être ramené dans les limites de l'expérience.)

Mais puisque Condillac ne tenait aucun compte des éléments perceptibles, qui pouvaient se rapporter à la motilité des organes, il dut nécessairement méconnaître la condition première dans laquelle se fonde la circonscription des parties du corps et en assimiler les moyens à ceux de toute autre connaissance objective. S'il était remonté jusqu'à cette condition, il aurait vu qu'il ne s'agit plus que de chercher dans ce domaine réel, quelle peut être la sensation, immédiatement relative par elle-même, qui communique ce caractère à tous les modes qui coïncident ou s'associent avec elle. Condillac croit trouver exclusivement un tel caractère dans la sensation de solidité dont la main est l'organe propre ; la main seule, s'appliquant d'une manière immédiate à l'objet solide, étendu comme elle, peut en reconnaître l'existence comme objet hors du $m o i$, en circonscrire les limites ; en déterminer, en créer, pour ainsi dire, les formes. C'est ainsi que les parties de notre propre corps, confondues dans le sentiment fondamental, se développent, s'étendent et se figurent en relief, sous le moule sensible qui les parcourt. Tant que le sentiment éprouve une réplique, c'est toujours le même moi qui se retrouve et se répond à lui-même ; dès que la réplique cesse ce n'est plus le moi, etc.

Je vois bien là un module universel, un instrument qui sert à connaître, à mesurer des objets, mais cet instrument lui-même comment est-il connu d'abord, car lui aussi est objet pour le moi qui commence à s'en servir, et avant qu'il l'ait encore appliqué à aucune autre partie, ni à rien d'étranger ? L'analyste ne remonte pas encore assez haut. Le caractère fondamental de relation extérieure, qu'il assigne à l'exercice particulier d'un sens, il l'a supposé déjà et il en a fait usage longtemps avant de mettre le toucher en jeu. Toutes les fois qu'il s'est agi, par exemple, de la locomotion ou de la direction d'un organe, tel que la vue, comment a-t-il pu ne pas en dériver quelque sensation relative?; comment le sujet de l'effort a-t-il pu se confondre entièrement avec le terme organique ?; comment un organe mobile quelconque a-t-il été constamment dirigé sans être connu ? il fallait donc que les actes et mouvements fussent aussi automatiques qu'ils peuvent l'être sous la loi de l'instinct. Mais alors quel est le fondement de toutes ces opérations intellectuelles dans la statue, bornée aux odeurs, aux couleurs, etc. ? Quel est avant tout, le fondement de sa personnalité, qui ne saurait être constituée pour elle-même, hors d'une relation quelconque ? Et s'il n'y a pas une personne identique, où va se rattacher la 
chaîne commune des modalités successives qui se transforment, où est le point d'appui de toute l'existence ${ }^{1}$ ?

Dès qu'il y a déploiement d'effort, il y a un sujet et un terme constitués l'un par rapport à l'autre, et toutes les impressions deviennent plus ou moins particulières ou, relatives, proportionnellement au degré de liaison, de proximité, de dépendance où elles sont à l'égard de ce mode relatif fondamental. Sans lui, tout est passif et absolu, les impressions du toucher comme les autres ; avec lui, tout se rapporte à une personne qui veut, agit, juge du résultat des actes, distingue, par le contraste, les modes forcés de la sensibilité passive de ceux qu'elle produit par un vouloir, et peut ainsi acquérir, soit directement par le toucher, soit par une sorte d'induction (dans l'exercice de tout autre sens) l'idée de quelque existence ou force étrangère, conçue sur le modèle de la sienne propre.

Il s'agissait donc de remonter jusqu'à ce mode fondamental premier, auquel la personnalité est essentiellement inhérente, ou dont le moi est indivisible, d'en rechercher les conditions générales ou particulières, de voir comment il peut concourir avec l'exercice de divers sens, ou en faire partie essentielle, composer ainsi les affections immédiates et les féconder en les élevant à la hauteur de l'idée. Avant de chercher le passage de nos sensations à la connaissance des objets extérieurs qui n'en sont pas toujours les causes, ni même les occasions, la métaphysique avait donc à résoudre une question première et plus générale, où était même renfermée cette dernière comme un cas plus particulier : c'était de s'informer avant tout du passage d'un état purement affectif (tel qu'il peut être conçu approximativement par plusieurs signes pris dans notre existence même) à celui d'aperception personnelle, qui est bien nécessairement le fondement, le premier degré de toute intelligence. Ce problème conduisait directement à chercher, et à trouver peut-être, la condition commune qui, servant à effectuer dans l'origine le déploiement d'une force vivante, sert aussi à circonscrire dans son domaine propre, et par autant d'actes relatifs de conscience, les termes multiples de son application, à distinguer ainsi, dans leurs limites réciproques, les termes inertes qui obéissent à la même puissance motrice, et ceux qui lui résistent sans obéir. Ainsi, la même cause, qui localise les impressions et constitue un moi qui les aperçoit à distance, localise aussi un objet lorsque le terme de l'effort s'éloigne davantage, ou que la résistance étrangère, l'inertie absolue, remplace l'inertie propre des organes et s'ajoute avec elle (ceci s'éclaircira mieux à l'article du toucher) ${ }^{2}$.

1 C'est du principe ou de l'origine du sentiment de causalité qu'il fallait s'occuper d'abord, parce qu'en fait ce sentiment, qui est celui de la personnalité même, une fois constitué, on le voit s'associer de diverses manières avec les différentes impressions, soit par un rapport de dérivation si ces impressions ressortent de la volonté, soit par un simple rapport de coexistence ou de simultanéité, si elles sont passives par leur nature.

2 La nécessité d'une influence motrice, ou d'un effort actuel exercé sur des parties sensibles, pour que les impressions faites sur ces parties puissent y être directement rapportées, me 
Sans doute les deux passages, dont il s'agit, ont pu être franchis dans le même temps; et les limites du moi ou de ce qui lui appartient en propre, n'ont pu nettement se circonscrire, que par la connaissance relative de ce qui est en dehors. Les deux connaissances personnelle et objective, ou les conditions et moyens qui les effectuent dans l'origine, ne sont point réellement séparées; mais il s'agit de savoir s'il n'y a pas une séparation possible ou seulement une

paraît confIrmé par un fait curieux rapporté dans un ouvrage peu connu intitulé Histoire naturelle de l'âme, par M. Rey REGIS, médecin de la Faculté de Montpellier). « Ayant vu, dit ce médecin, un malade qui paraissait paralysé de la moitié du corps, après une attaque récente d'apoplexie, je fus curieux de savoir s'il lui restait quelque sentiment et quelque mouvement dans les parties affectées. Pour cela, je pris sa main sous la couverture du lit, et pressai fortement un de ses doigts, ce qui lui fit jeter un cri ; en ayant fait autant à chaque doigt, il sentit chaque fois une douleur très vive, mais sans la rapporter nulle part. Je mis alors ma main dans la sienne et lui dis de me serrer, il ne le put, etc. Cet homme eut besoin de plusieurs jours d'expérience pour apprendre de nouveau à se servir de sa main, à remuer les doigts les uns après les autres, et dès lors aussi, il sut rapporter parfaitement la douleur au doigt pressé. » L'auteur conclut que, dans des paralysies de ce genre, l'âme perd la connaissance ou le souvenir de sa force motrice, de la proportion de son effort au mouvement requis, ce qui revient à dire que le sujet de cet effort moi perd l'idée ou le sentiment immédiat de celui des termes particuliers de son application, qui se trouve organiquement lésé. Et si tous les termes partiels, ou le corps en masse était dans même état, toute aperception ne se trouverait-elle pas complètement suspendue, comme dans le sommeil, quoique l'affectibilité passive pût subsister? Le rapport direct des impressions à un siège tiendrait donc originairement aux mêmes conditions que la motilité volontaire ; il pourrait donc être séparé de la partie affective. Or, si l'on reconnaît cette séparation dans le premier sans doute de tous les jugements, il faudra bien l'admettre dans les autres; ne sommes-nous pas d'ailleurs, quant aux impressions des organes internes, dans le cas du paralytique de M. Régis ?

Condillac n'a point cherché en aucune manière comment le moi pourrait acquérir directement la connaissance intérieure des organes ; il ne s'occupe que d'une connaissance objective et secondaire de leurs formes extérieures. Autrement, il aurait vu que la réplique de l'effort, dans des organes soumis à la même volonté, a le pas avant la réplique du sentiment, comme le confirme le fait précédent. Cette observation première en aurait amené bien d'autres contraires à plusieurs résultats du Traité des sensations, notamment celle-ci que je trouve à l'article du toucher : La main franchissant des parties intermédiaires du corps (propre) qu'elle parcourt, se retrouvera dans chacune comme dans autant de corps différents et ne saura pas encore que toutes ensemble n'en forment qu'un seul, parce que les sensations qu'elle a éprouvées ne les lui représentent pas comme contiguës ou formant un seul tout continu. » Non sans doute, la main ne le sait pas, mais l'individu le sait, s'il a quelque sentiment fondamental ou quelque aperception d'existence, s'il est capable de se locomouvoir et de diriger ses organes ou son corps en masse, s'il n'est pas enfin sous la loi de l'instinct, exclusive de toute intelligence. La connaissance de la forme, des dimensions, de l'étendue des différentes parties du corps, mesurées par la main aidée de la vue, diffère bien sûrement de cette connaissance intime, relative au déploiement du même effort sur un terme inerte, qui est inséparable de l'existence même du moi, et qu'on suppose nécessairement dès qu'on admet un sujet individuel à qui les modes et les actes se réfèrent. Cette distinction ressort même clairement du dernier passage pris du Traité des sensations. Je ne finirais pas si je voulais noter tous les résultats comparés de ces deux points de vue fondamentaux; mais je crains de paraître déjà insister beaucoup sur des objets qu'on n'aime guère ordinairement à creuser. 
distinction réelle et intelligible; il s'agit de savoir, si pour fixer les limites des deux domaines de moi et de non-moi, nous prendrons notre point de départ en dehors ou en dedans, si nous remonterons de l'existence supposée des objets et des impressions dont ils sont censés être les seules causes actives, à la connaissance ou à l'aperception personnelle, indivisible, par hypothèse, de ces impressions passives; ou si nous descendrons au contraire de cette connaissance intérieure (et des conditions qui lui sont propres) à la perception des objets et des qualités ou modifications qui s'y rapportent, en distinguant toujours ces deux ordres de connaissance, et leur mode de génération par les sens ; en un mot, si, dans le rapport primitif de causalité sur lequel toute perception se fonde, nous prendrons pour antécédent une force extérieure sous laquelle nous sommes passifs, ou une puissance toute intérieure qui prédomine les objets mêmes ${ }^{1}$. De ce premier pas dépend tout progrès ultérieur, et le choix de l'une ou l'autre alternative décide du reste. Je n'ai pas besoin de justifier ici l'ordre qui me paraît établi par la nature même du sujet pensant, dans les progrès de la connaissance, et la distinction des deux espèces de cette connaissance. Tout ce qui précède et tout ce qui suit parle assez pour ou contre le parti que j'ai cru devoir adopter.

II. - Le principe de la vie de relation et de conscience, une fois en activité et en pleine possession de son domaine, se reproduit constamment sous une même forme. Son exercice périodiquement suspendu, pendant que celui de la vie absolue ne s'interrompt point, le fait renaître toujours égal ; c'est toujours la même force qui lutte contre le même terme résistant, il ne peut y avoir là de variations que dans le degré.

La veille du moi est constituée par cet état d'effort immanent. Tant qu'elle subsiste, la personne est plus ou moins, mais toujours identiquement présente à elle-même; alors et hors des vives exacerbations de la sensibilité qui obscurcissent quelquefois l'aperception personnelle, les impressions successives de toute nature coïncident avec le même mode fondamental, et peuvent participer, quoique très inégalement, à la lumière de la conscience. Mais les unes ne font réellement qu'y participer, et dans le plus faible degré, à raison de leur éloignement de la source de toute lumière; les autres s'en rapprochent davantage, et viennent se teindre de ses rayons; d'autres enfin l'apportent avec elles ou sont irradiées de la même source.

$1^{\circ}$ Dans la première classe se rangent toutes les impressions purement passives de la sensibilité, dont le siège est entièrement hors des limites de la puissance motrice, et toute la force impulsive ou réactive dans les organes de la vie intérieure, ou le centre qui leur est sympathiquement uni. Ces affections organiques ou animales, dans le plus bas comme dans le plus haut degré, ne sont point par elles-mêmes dans la conscience ; elles forment en quelque sorte

1 Ne plagis omnia fiant externa quasi vi (Lucret, Voyez l'épigraphe). 
la matière de la sensation, ou plutôt, comme dit Locke, de l'idée de sensation; mais aucune idée proprement dite ne peut émaner exclusivement de cette source. Telles sont toutes les impressions qui naissent directement du bon ou mauvais état de la machine ; celles qui se rapportent uniquement à l'appétit, etc. Il faut observer que ces affections ne s'associent, pour ainsi dire, que collatéralement avec le mode fondamental ou avec ceux qui dérivent de notre activité, mais sans entrer en combinaison intime avec eux, sans faire partie des mêmes composés perceptifs. Cela paraît bien, en ce que de telles affections ne sont jamais directement rapportées à un siège déterminé, et sont toujours hors de la sphère du jugement comme du souvenir. Dans tous les cas, le nom de sensations ne peut leur être qu'improprement appliqué, et il me paraît nécessaire de les distinguer sous celui d'affections que je leur conserverai.

$2^{\circ}$ La seconde classe comprend les impressions faites sur les sens externes par des causes étrangères. Les organes de ces sens ayant chacun un mode d'affectibilité qui leur est propre, mais participant d'un autre côté, quoique dans des degrés différents, à l'action d'une même force motrice qui les active, leurs fonctions sont susceptibles d'être considérées sous ces deux rapports.

A. Lorsque l'affectibilité, mise en jeu par la cause extérieure, est prédominante, la matière de la sensation prévaut sur l'acte perceptif, toujours inhérent au déploiement de l'effort. Cette prédominance est bien exprimée par le terme sensation qui convient alors à cette classe. On reconnaît ici un véritable composé et deux éléments qui se rapportent à deux sources différentes, mais qui sont intimement unis dans le même siège, et non pas seulement agrégés, pour ainsi dire, comme dans le cas précédent, c'est à ce point aussi qu'on peut commencer l'analyse de décomposition. Comme dans l'état de veille, les organes particuliers de ces sensations sont plus ou moins tendus par la volonté, et participent directement à l'effort général, elles sont toujours, hors les cas extrêmes, accompagnées de quelque aperception rapportée à leurs sièges, et composées de divers jugements d'expérience. Mais cette expérience a eu un commencement, elle repose sur certaines conditions qui l'ont rendue possible. Or, ces conditions ne sont point inhérentes à ce qui fait ici la matière de la sensation, et lorsqu'on la conçoit séparée de la forme, toute lumière disparaît.

B. L'organe peut être tellement constitué, et l'agent externe auquel il est soumis, d'une telle nature, que les impressions reçues soient très peu affectives d'une part, pendant que l'effort est comme inaperçu dans son déploiement peu intense, d'autre part. Il y a là un certain rapport entre les deux forces, qui ne permet guère d'assigner quelle est celle à qui appartient l'initiative ou la prédominance. La nullité d'affection directe exclut le caractère sensitif, la faiblesse de l'action motrice exclut presque le caractère aperceptif, l'individu ne sent ni n'agit et pourtant le phénomène de la représentation s'accomplit, il y a un objet extérieur ou intérieur passivement perçu. C'est ici que l'idée de 
sensation paraît exister par elle-même et venir toute faite du dehors; ce phénomène mérite bien d'être analysé dans ses circonstances et conditions particulières; nous le distinguerons sous le nom de perception simple ou objective (intuition).

C. Il y a un mode de sensations où la volonté motrice prend essentiellement l'initiative, quoiqu'elle ne conserve pas toujours la prédominance sur la force extérieure. Le sens va chercher son objet qui ne vient point tout seul au devant de lui pour le provoquer. Ici la perception objective, activée expressément par le vouloir renferme l'aperception dans l'acte même qui se distingue de son résultat, et l'analyse n'a aucune peine à y signaler deux éléments. Ici l'on trouve encore le premier fondement de deux sortes d'observations. Cette manière de percevoir doit être distinguée de la précédente par son caractère constitutif; nous l'appellerons perception active (ou aperception externe médiate).

D. Il est enfin une espèce de sensations que la volonté produit seule, sans le concours d'aucune force étrangère, et en fournissant elle-même, pour ainsi dire, la matière et la forme. Ici est le mobile premier et la source pure de la réflexion ${ }^{1}$.

La distinction réelle à établir entre les sens est donc toute fondée sur la dépendance où se trouve plus particulièrement placé chacun d'eux, en vertu de sa constitution, à l'égard de deux forces, l'une en dehors, l'autre en dedans du moi. Ces forces peuvent agir séparément dans tels sens particuliers, plus souvent elles concourent à leur exercice. Mais tantôt elles s'équilibrent, tantôt elles se prédominent de diverses manières, de là des produits essentiellement divers, que le terme sensation peut bien exprimer génériquement, mais en laissant confondus les titres des espèces. De là aussi deux ordres de facultés et deux modes de leur génération. Il ne suffit pas de dire que tout vient des sens, il faut savoir encore comment et par quel canal les idées et les opérations intellectuelles peuvent en venir ; autrement, on ne fait guère, si je puis ainsi parler, que l'ontologie de la sensation, au lieu d'en faire l'analyse ; mais cette analyse peut encore suivre deux méthodes différentes indiquées par la division même que nous venons d'établir. On peut prendre, par exemple, un sens quelconque pour type générateur; et en ayant égard aux deux sortes de fonctions qui concourent à son exercice, trouver ainsi une division réelle des produits, modes ou opérations élémentaires qui se rapportent, en effet, à cette source mixte. Mais comme l'espèce et les caractères des éléments, ainsi assignés, dépendent de la nature de l'une des deux forces prédominantes dans le sens qui a servi de modèle, il serait dangereux et souvent illusoire de généraliser trop promptement ces caractères particuliers, ou de les affirmer des

Cette division relative aux fonctions des sens se trouvant reproduite dans un tableau qui est à la fin de cette partie du Mémoire, ne devait pas entrer ici où elle ne peut servir qu'à annoncer ce qu'on verra ailleurs avec plus de précision. 
autres sens qui ont aussi leurs produits élémentaires, lesquels ne peuvent être appréciés ni connus si l'on ne remonte jusqu'à cette origine spéciale. L'analyse des facultés intellectuelles n'est donc pas celle de la sensation en général, mais bien celle de chaque sens en particulier, des conditions et circonstances qui forment son exercice, des produits qui en dérivent, etc.

On pressent néanmoins que si cette méthode nous offre d'une part une multitude effrayante de distinctions et de variations à noter dans le jeu des organes et les modes passifs qui s'y réfèrent immédiatement, elle doit nous offrir, sous le côté qui est le plus rapproché de notre point de vue intérieur, beaucoup plus d'uniformité et de constance, de telle sorte que nous nous trouvions fondés ensuite à étendre à l'exercice commun des sens les titres et les caractères des modes actifs signalés dans l'un d'eux, non en donnant à ces titres une valeur générale ou commune, mais en leur conservant l'individualité précise qu'ils ont toujours dans notre réflexion (voyez la première partie). C'est dans un tel point de vue que nous allons en entreprendre l'analyse.

C'est dans le but d'une distinction et d'une dérivation de ces facultés intellectuelles que nous allons entreprendre l'analyse suivante et refaire dans un nouveau point de vue le Traité des sensations. 


\title{
Chapitre II
}

Continuation du précédent.

\begin{abstract}
Analyse et dérivation des facultés et des idées élémentaires qui peuvent se rapporter à l'exercice d'un sens particulier. - Premier exemple pris de l'odorat.
\end{abstract}

$\underline{\text { Retour à la table des matières }}$

L'entreprise de reconstruire, pour ainsi dire, l'entendement humain avec certains éléments artificiels hypothétiques (donnés souvent par les seules classifications de notre langage) ne serait peut-être guère moins téméraire que ne le serait celle de recomposer chimiquement un produit naturel du règne végétal ou minéral, altéré et résous dans ses éléments par le creuset.

La nature qui seule dispose de deux grands instruments, le temps et l'espace, peut seule aussi combiner et agencer dans des touts parfaits les matériaux élémentaires qu'elle a lentement préparés à s'unir, par une infinité d'élaborations successives et imperceptibles. En appliquant nos sens, nos instruments, à ces composés extérieurs, comme notre réflexion ou nos hypothèses à cet autre composé qui est nous-mêmes, nous les brisons, nous saisissons quelques-uns des fragments épars les plus grossiers, mais la puissance agrégatrice nous manque, et les éléments, qui ont nécessairement échappé à nos analyses, ne sauraient entrer dans nos compositions artificielles.

Il faut le dire : indépendamment des difficultés extrêmes attachées à un projet, tel que l'avait conçu le célèbre auteur du Traité des sensations, le point de vue où il se plaça, dès son début, devait en rendre l'exécution impossible ou tout à fait illusoire. 
Pour qu'un être soit impressionnable ou affectible par un sens quelconque, il faut bien d'abord qu'il vive ; or, il ne peut vivre hors du concours de divers instruments organiques plus ou moins nombreux, qui se correspondent solidairement et font, pour ainsi dire, un échange perpétuel de leurs impressions. Cette vie commune est inséparable d'un sentiment fondamental absolu, qui est bien modifié par les impressions accidentelles du dehors, mais non constitué par elles, puisqu'il leur donne le ton au lieu de le recevoir. Dans le point de vue physiologique, l'hypothèse de Condillac serait donc inadmissible ; mais en prenant la chose d'un autre côté, admettons que le sentiment général absolu qui fait la vie de l'être fictif (et que l'on peut dès lors considérer comme réel) dont il s'agit, soit modifié, suivant le ton approprié à une odeur de rose, nous pourrons dire en effet qu'il la devient, et cette expression nous représente très fidèlement l'une des conditions de l'existence sensitive absolue, et non encore relative à un sujet individuel et permanent qui l'aperçoive ${ }^{1}$.

Mais mieux je saisis ainsi cet état affectif, pour ainsi dire, dans sa pureté originelle, moins je conçois qu'on puisse dériver d'une telle source exclusive, et par simple transformation, quelques modes intellectuels de nature égale à ceux dont nous retrouvons le type complet en nous-mêmes. Or, c'est précisément de ceux-là qu'il est question, c'est notre intelligence, notre être pensant tel qu'il est, ou s'apparaît à lui-même dans sa propre réflexion, qu'il s'agit de reconstruire s'il est possible, et non un fantôme hypothétique, que nous ayons à habiller ou à créer.

Si nous sommes obligés de feindre des hypothèses pour atteindre la réalité et en vue de la réalité seulement tâchons du moins de les rapprocher le plus possible de la nature.

Supposons donc aussi un être vivant de notre espèce, doué de toutes les conditions de l'existence sensitive absolue et par conséquent irréductible à une seule espèce d'impressions accidentelles venues du dehors, qui puissent être censées constituer cette existence ; prenons-le au sortir de l'instinct, au moment où l'ordre des mouvements va changer, où l'influence d'un centre d'action directe de la vie de conscience va succéder à celle du centre de réaction

\footnotetext{
« Approchez, dit BONNET (Essai analyt., chap. VI, § 35) une rose du nez de la statue; au même instant elle devient un être sentant, son âme est modifiée pour la première fois, elle est modifiée en odeur de rose, elle devient une odeur de rose, elle aperçoit, se représente cette odeur ; toutes ces expressions sont synonymes. » Non pas, s'il vous plait, car l'acte d'aperception et la représentation surtout suppose un sujet et un objet qui ne sont point identifiés, mais très distincts dans la pensée. Or, c'est précisément une telle distinction qui se trouve exclue par le terme propre, et pour ainsi dire technique devient. Bonnet qui avait été précédé par Condillac dans l'exposition d'une hypothèse sur le choix de laquelle ils s'étaient rencontré, emprunte de lui cette expression sans en avoir, je crois, bien pesé la valeur.
} 
sympathique d'une vie toute intérieure ${ }^{1}$, par suite au moment où l'affection simple va revêtir la forme de la sensation; écartons pour un instant toute occasion externe d'impressions autres que des odeurs, ou supposons tous les sens, hors l'odorat, momentanément paralysés pour le sentiment, quoique les conditions d'un effort général sur le corps en masse subsistent ; faisons enfin abstraction du rapport sympathique, qu'entretiennent surtout certaines odeurs particulières avec les organes de la sensibilité intérieure, pour considérer ces sensations dans un autre ordre de produits dérivés, et cherchons à obtenir, sous une forme hypothétique, des résultats que l'observation intérieure puisse justifier.

\section{I - Origine des facultés actives.}

\section{Retour à la table des matières}

L'odorat est essentiellement lié à la respiration qui commence avec la vie, et ne finit qu'avec elle. Cette fonction est d'abord purement organique et, tant qu'elle s'accomplit uniquement sous la loi de l'instinct, elle demeure, pour l'être vivant qui s'ignore, aussi obscure dans ses effets que dans son principe. Les impressions d'odeurs, qui peuvent être liées à ces mouvements respiratoires instinctifs, ne sortent point du cercle des affections simples; il n'y a point de personne qui les aperçoive ou les sente ; la combinaison organisée les devient comme tout le reste, c'est elle qui respire. Mais lorsque cette dernière fonction a passé avec d'autres mouvements sous l'empire de la volonté, qui concourt du moins à la modifier, sinon à la produire, elle s'approprie dès lors comme acte à l'aperceptibilité et la connaissance, elle devient même, par ses résultats, un moyen de connaissance; d'où il suit que si le mouvement respiratoire instinctif est un accessoire indifférent à l'effet de l'impression d'odeur, ce mouvement converti en effort volontaire d'odoration en est, une circonstance et même une condition très notable, puisque cette impression peut avoir des caractères tout à fait différents, suivant qu'elle est séparée ou accompagnée de ce mode d'effort qui peut être même nécessaire pour la produire ${ }^{2}$

Cela posé, nous concevrons qu'au sortir de l'instinct, lorsqu'un sujet commun a été constitué dans le rapport de la force à la résistance organique, et avant même qu'il n'y ait rien de connu au dehors, une impression d'odeur qui coïncidera pendant la veille avec l'effort général (quoique non encore lié expressément avec l'acte inspiratoire, supposé inaperçu) aura quelque caractère de plus que celui de simple affection; l'individu ne la deviendra pas

Voyez le chapitre II de la section précédente.

Voyez chap. II, ${ }^{\text {re }}$ section. 
entièrement, mais il pourra être dit la sentir, à peu près comme nous sentons nous-mêmes une douleur intérieure qui ressort, pour ainsi dire, du sentiment fondamental et absolu de l'existence.

Mais supposons qu'un effort d'inspiration, élevé au-dessus du ton instinctif ordinaire, soit déterminé d'abord par une cause quelconque, l'individu sentira cet acte en résultat, non comme tout autre mode permanent passif ${ }^{1}$, mais comme un mouvement lié en partie au déploiement de la force qui le constitue $m o i$, il peut le répéter dès lors par une détermination proprement volontaire, pour exercer sa puissance, s'imiter lui-même, et sans y être forcé par de vives affections qui effaceraient tout vouloir ; en changeant l'ordre des mouvements ${ }^{2}$ dans cet exercice répété, le terme de l'effort inspiratoire peut se circonscrire, et l'individu se préparer à sentir les impressions comme lui venant par cet organe, à les transformer ainsi en véritables sensations.

Pendant que l'action d'inspirer s'accomplit de cette manière, plaçons à distance une fleur dont l'odeur agréable ne puisse être sentie ou parvenir à l'individu qu'à la suite d'un effort soutenu d'inspiration, il se trouvera ainsi modifié par le résultat d'un acte dont il dispose; mais tout entier à cette nouvelle sensation qui devient alors le mobile et la cause déterminante, de l'acte continué et renforcé, il pourra cesser d'apercevoir ce dernier, et ignorer par conséquent que la sensation en elle-même en est une dépendance partielle.

Cependant l'effort s'affaiblit, revient à son ton ordinaire et l'odeur s'évanouit, l'action recommence et la sensation avec elle, tant que les choses demeurent au dehors dans le même état. L'être moteur et sentant n'ayant actuellement, par l'hypothèse, aucun moyen de connaître ni de soupçonner qu'une cause extérieure agit sur son organe pour le modifier, mais ayant l'aperception immédiate de l'effort et par lui du résultat affectif qui le suit ou l'accompagne, devra s'accoutumer, après quelques répétitions et expériences ${ }^{3}$ de ce genre, à lier ensemble l'acte et son produit dans un seul et même mode, qu'il attribue à sa puissance ou à sa force constitutive. Le mode actif d'inspiration se trouvant comme identifié avec l'impression reçue du dehors, il voudra celle-ci comme il veut et parce qu'il veut l'autre, il sera pour lui-même comme s'il exerçait sur tous deux un pouvoir égal. Je dis pour lui-même parce qu'il y a ici deux points de vue très distincts pris, l'un en dedans, l'autre en dehors de l'être dont il s'agit; il importe de bien noter cette différence avant d'aller plus loin. Si nous considérons ce qu'est un tel être par rapport à lui, en nous mettant à sa place, autant qu'il est possible dans notre hypothèse, il sera pour nous, ou nous serons en lui, une puissance qui veut et produit telle

Voyez chap. II, $\mathrm{I}^{\mathrm{re}}$ section.

Nous verrons bientôt, que l'acte peut recommencer par une, double détermination.

En admettant une personne douée de quelque degré d'aperception, nous avons la condition fondamentale de toute expérience, le passage immédiat de la loi de l'instinct à celle de l'habitude exclut l'expérience réelle. 
modification sensitive, comme elle produit le mouvement associé dont elle dispose. Si nous le jugeons avec nos yeux du dehors, il ne sera plus pour nous qui connaissons parfaitement sa passivité, sous l'action de la cause odorifère dont nous nous servons pour l'impressionner, il ne sera plus, dis-je, qu'une simple vertu sentante, qu'une sorte d'automate organisé de manière à recevoir des impressions, et à réagir nécessairement en conséquence pour en amplifier les effets. Alors cette réaction organique, étant prise pour signe de la volonté, est bien subordonnée à une action première qui a commencé au dehors. La volonté même ne diffère donc point, dans son principe, de la sensibilité, et s'identifie avec le désir, avec les plus aveugles déterminations de l'instinct.

Mais, en prenant les faits uniquement dans la conscience de l'être sensible et moteur, il en est tout autrement. D'abord, fût-il encore plus passif relativement à nous, sous l'impulsion d'une cause étrangère modifiante, dès qu'il ne peut avoir aucune connaissance de cette cause, elle est pour lui comme n'existant pas. D'un autre côté, il suffit qu'il ait le sentiment intérieur d'une puissance moi, déployée librement dans certains cas, et non exercée dans d'autres, pour que la distinction qui s'établit au-dedans de lui, par le contraste entre un état actif de vouloir et état passif d'affectibilité, ait le fondement le plus réel, le seul qu'il soit possible d'obtenir d'une part, et impossible de nier de l'autre, à moins qu'on ne se place dans un point de vue tout à fait extérieur à l'être dont il s'agit, ou qu'on ne prétende juger de ce qu'il est absolument sans tenir aucun compte de ce qu'il est pour lui-même ${ }^{1}$.

Rien n'est plus propre à montrer la différence de ces deux points de vue et à tenir en garde contre les mésentendus auxquels cette différence peut donner lieu dans la science des facultés de l'être pensant, que la manière dont Condillac distingue les deux états actif et passif de la statue. «Elle est active, dit-il, quand la cause qui la modifie est en elle-même (ou dans son organisation) ; elle est passive, quand cette cause est extérieure. » Je dirai, moi, qu'elle est active toutes les fois qu'elle est ou se sent comme cause ou force productive de certaines modifications, et passive, toutes les fois qu'elle est modifiée sans (ou même contre) le déploiement d'une telle force. D'ailleurs, si l'on n'avait égard qu'à ce que peut être la statut pour elle-même, comment pourrait-on établir, par rapport à elle, quelque distinction solide fondée sur l'extériorité d'un objet ou d'une cause, qu'elle est censée invinciblement ignorer?

La statue serait-elle donc active quand elle éprouve actuellement une douleur intérieure et passive, quand elle éprouvera dans la suite une sensation de solidité ?

C'est aussi pour avoir voulu se placer tout à fait en dehors des faits de conscience, et juger ainsi extérieurement de ce qui peut être avant la première détermination du vouloir, avant le moi lui-même, que les métaphysiciens me paraissent avoir élevé tant de questions oiseuses et interminables au sujet de sa liberté, question qui ne peut trouver de réponse hors du sentiment intérieur de nos actes et qui devient nécessairement frivole, par cela même qu'on l'agite. Je citerai un exemple qui me paraît assez propre à justifier la méthode que je crois exclusivement appropriée à la science de nos facultés.

« Ne comprenez-vous pas clairement, dit BAYLE (Questions d'un provincial, p. 764) qu'une girouette à qui l'on imprimerait tout à la fois le mouvement vers un certain point de l'horizon et le désir de se tourner de ce côté, serait persuadée qu'elle se mouvrait d'ellemême pour accomplir ses désirs ? (je suppose qu'elle ne saurait point qu'il y a des vents et qu'une cause extérieure fait changer tout à la fois sa situation et ses désirs); nous voilà 
En nous en tenant à ce dernier point de vue, l'être, qui n'odore qu'autant qu'il agit et qu'il veut le mouvement inspiratoire, est donc également actif pour lui-même, et dans la production de ce mouvement et dans le mode affectif qui l'accompagne (par hypothèse) d'une manière constante et invariable, quoique ce dernier dépende par le fait objectif (ou relativement à notre point de vue extérieur) d'une cause étrangère qui concourt à le produire, et que le mouvement lui-même puisse se rattacher encore, sous le dernier rapport, à quelque circonstance organique inconnue, à quelque être invisible qui effectue le débandement d'un ressort et de l'activité matrice, etc., car tout cela ne change rien ni au vouloir ni au sentiment réel du pouvoir.

Je dis donc que notre être fictif voudra l'odeur, comme le mouvement même, tant qu'il n'aura pas encore eu l'occasion de sentir, par quelque contraste, qu'il est subordonné dans la sensation à une puissance différente de celle qui produit l'effort. Ainsi l'idée d'un but, auquel tend le vouloir, ne sera point distincte dans le cas présent de ce vouloir même, comme elle l'est pour nous qui, connaissant, des causes modifiantes extérieures, dirigeons vers elles toute notre action, les appelons, quand elles sont éloignées, de tous nos désirs ou nos vœux, et employons, quand elles sont présentes, tous les moyens disponibles pour nous mettre à portée de recevoir leur entière influence. Or, ces moyens ne sont autres que des mouvements volontaires ${ }^{1}$ et la puissance

naturellement dans cet état, nous ne savons point si une cause invisible ne nous fait pas passer successivement d'une action ou d'une pensée à une autre, etc. »

Bayle admet, dans cet endroit, l'opinion des Cartésiens comme la plus favorable aux doutes sceptiques, dont il prétend envelopper le sentiment de nos actes volontaires ou libres (voir la note sur Hume) : savoir que ce n'est point l'âme ou le moi pensant qui exécute ses vouloirs, qui meut les organes, etc. Aussi, c'est-il le désir qu'il suppose toujours pouvoir être effectué comme le mouvement même par une cause invisible. Mais le désir est-il donc identique avec le vouloir, le sentiment ou l'idée du pouvoir se trouve-t-il essentiellement compris dans l'un comme dans l'autre ? Or, d'où vient cette idée (de pouvoir) ? pourquoi se réfère-t-elle à certains actes et non à d'autres ? existerait-il un être voulant, s'il n'y avait pas un être moteur ? Ôter au sujet pensant l'exécution réelle des actes dont il est assuré par conscience, n'est-ce pas anéantir avec le sentiment de sa puissance celui même de sa personnalité ? et si c'était Dieu qui exécutât les propres mouvements que nous nous attribuons, ne serions-nous pas complètement identifiés avec lui ou lui avec nous ? On peut remarquer, au surplus, l'analogie de l'hypothèse de Bayle et de celle que j'emploie. Quoique pour l'être qui n'exercerait d'autres sens externes que l'odorat, la cause modifiante fût aussi inconnue que le vent l'est à la girouette, le sentiment de sa propre causalité, dans l'effort inspiratoire librement déterminé, n'en serait pas moins un fait réel de conscience, un fait primitif du sens intime dont on ne pourrait demander l'explication.

1 Je sais bien que chaque partie de l'organe sensitif s'étend aussi, s'avance, se tuméfie comme pour chercher la cause appropriée à son affectibilité spécifique, ou se mettre entièrement à sa portée, et c'est là une image de ce qui se passe sous la loi de l'instinct, dans la combinaison organisée entière ou le tout vivant; mais comme ce jeu d'affinités animales s'accomplit entièrement sans conscience et dans des cas où nous ne pouvons absolument supposer qu'il y ait de moi, plus on rapproche le désir de semblables modifications organiques ou plus on l'identifie avec les déterminations purement instinctives, plus aussi on le sépare de ce mode fondamental de notre existence individuelle et aperçue que nous nommons vouloir ; le désir, en un mot, considéré surtout dans les limites où il se confond 
que nous appelons volonté, étant tout entière dans la production immédiate infaillible, s'arrête là sans aller plus loin.

Le, désir, au contraire, activé uniquement par l'objet ou son image, s'étend au dehors, et l'être qui le désire se sent par là même constitué en rapport de dépendance d'une cause étrangère qui cède ou résiste. L'individu supposé ne connaissant encore rien hors de sa puissance constitutive et de la modification infailliblement associée jusqu'à présent à son exercice, n'aura donc que des vouloirs, tant que rien ne changera autour de lui, il sentira comme il mouvra par un seul et même acte, et le désir n'aura pas le temps de naître.

Cependant, l'intimité d'association supposée entre le mode actif de l'effort inspiratoire et l'impression d'odeur qui le suit, tant que la même cause (ignorée) demeure présente, cette intimité dis-je, ne saurait être conçue permanente et invariable, sans forcer toutes les hypothèses ; les deux éléments de la sensation, appartenant à deux sources différentes, ne pourront longtemps demeurer entre eux dans un parfait et constant équilibre. L'effort et la cause extérieure restant les mêmes, les dispositions sensitives ou la capacité impressionnable de l'organe viendront nécessairement à changer dans un certain intervalle, et feront passer la modification par divers degrés de force ou de faiblesse. Dès lors les éléments du composé peuvent commencer à ressortir l'un hors de l'autre, et l'individu absolument simple dans la vitalité, simple aussi d'une autre manière dans l'exercice de son activité, peut reconnaître qu'il est double, ou qu'il y a en lui, dans ce qui le constitue (sujet individuel permanent) une source de constance, et hors de lui ou dans le terme organique de son action, une source de changements; dès lors aussi il apprend à rapporter à ce dernier l'élément qui varie ; car quoiqu'il le connût auparavant comme terme de l'effort, il ne pouvait (ne) pas le reconnaître comme impressionnable, tant que la modification sensible était indivise de l'acte, ou qu'il n'apercevait l'une que dans l'autre, en les attribuant également à une puissance moi qui ne se localise point ${ }^{1}$, dès lors enfin naissent, pour le même sujet moteur et sentant, deux ordres de facultés, des actes qu'il aperçoit en les produisant et des modifications qu'il éprouve sans les produire.

A. Origine de la réflexion et de l'attention. - Quoique par l'hypothèse (que nous continuerons encore sous la même forme), la sensation d'odeur, dont la

avec les premiers besoins, est aussi distinct de la volonté que la sensation animale l'est de la perception et cette distinction est fondée des deux parts sur la même base. (Voyez chap. II, I ${ }^{\text {re }}$ section.)

«Nous rapportons bien aux pieds, dit un philosophe (D'ALEMBERT Mélanges de littérature), la douleur (comme le mouvement effectif) mais nous ne leur rapportons point la volonté de marcher. »C Cette observation est très juste, et, en l'approfondissant, il me semble qu'on peut en faire ressortir bien des vérités sur lesquelles nous sommes trop enclins à nous faire illusion. Il en résulte, par exemple, que la volonté n'étant pas différente du moi, ce moi ne peut absolument pas se concevoir comme objet, comme chose, ou comme un composé d'organes. 
cause agit de loin et faiblement sur l'organe, demeure toujours subordonnée à un effort inspiratoire au-dessus du ton naturel de la respiration, les dispositions variables de l'organe peuvent néanmoins devenir telles, dans certains cas, que la modification s'avive ou s'exalte par les plus faible degré de cet effort volontaire. Quelquefois, au contraire l'action la plus énergiquement soutenue ne déterminera qu'une affection très obscure. Dès que nous supposons une personne constituée, il est impossible qu'elle confonde deux états ou deux modes d'existence aussi différents, et, si elle était capable de se faire un langage, elle ne les noterait pas probablement par le même signe. Lorsque l'impression, altérée en quelque sorte par l'organe, déjà monté de lui-même sur un certain ton sensitif, suit immédiatement, si elle ne prévient pas encore, l'acte le plus léger d'une volonté naissante, la conscience de l'individu est presque tout entière à la modification affective. L'acte de son vouloir disparaît ou s'obscurcit, tant par la promptitude et la faiblesse de l'effort, que par la vivacité relative de l'impression; le résultat seul persiste comme sensation; mais par cela que cette sensation est le résultat d'un acte, le moi ne peut être complètement identifié avec elle comme avec le vouloir seul, il ne la devient pas non plus, ou n'y est pas enveloppé comme dans une affection purement passive. Au contraire, lorsque l'impressionnabilité de l'organe sensitif se trouve rabaissée au-dessous du ton ordinaire, il faudra un déploiement d'effort très énergique, pour que l'émanation odorante puisse parvenir au sens, qui n'en sera que légèrement effleuré. C'est donc alors l'acte même qui ressort de la sensation ; et l'élément affectif, s'obscurcissant à son tour, n'est aperçu que dans le rapport de sa subordination à la puissance qui concourt à l'effectuer.

B. Passage de l'aperception à la réflexion. - En nous mettant à la place de l'individu supposé, et lui prêtant nos signes, nous disons qu'il aperçoit sa propre force ou qu'il s'aperçoit lui-même dans une simple détermination volontaire, séparée de tout mode sensitif résultant. Ainsi, dans l'hypothèse présente, il y a aperception personnelle dans le seul mode d'effort inspiratoire, séparément de toute odeur sentie, il y a un fondement au mot je et au verbe $j^{\prime} a g i s$, synonyme de j'existe, je m'aperçois existant.

Aperception immédiate interne et médiate externe. - Lorsque cet effort ou cet acte est suivi ou accompagné d'un mode quelconque, que l'individu attribue uniquement à sa puissance, mais en distinguant le résultat de l'acte lui-même qui prédomine simultanément dans la conscience, nous appellerons réflexion cette aperception redoublée dans le mouvement d'une part, et son produit sensible de l'autre (tel serait le fondement de cette formule employée par un grand maître, je sens une sensation, une idée, un rapport), etc. Tel est le lien de la modification passagère au sujet permanent je.

C. De l'attention. - Quand le sentiment propre de l'acte se cache ou s'obscurcit sous celui du résultat sensible (modal ou objectif) qui prédomine dans la sensation, l'élément de celle-ci provenant d'une force étrangère (connue 
ou inconnue), dont l'action ne commence point hors de la volonté, mais concourt toujours essentiellement avec elle et peut même se continuer hors de son influence : nous donnerons le nom d'attention à la force qui détermine et transforme ainsi la sensation, en ne s'apercevant plus elle-même que dans le résultat transformé. L'attention exclut la passivité complète, car le mode affectif n'a pas en lui-même la force transformatrice ; mais le caractère passif entre toujours plus ou moins en résultat dans l'exercice de la puissance ou la faculté que nous nommons ainsi, tandis que la réflexion est éminemment et exclusivement active.

Nous verrons mieux, en avançant, que les signes, d'après lesquels nous caractérisons ici ces facultés originelles et élémentaires, ne sont pas purement hypothétiques et conventionnels. Après les avoir prises ainsi dans leur source et leurs conditions les plus simples, cherchons à signaler de la même manière celles qui en dérivent ou qu'on peut considérer comme les transformées.

D. Mémoire et imagination. Différentes espèces de réminiscence. - Dans les intervalles, où la force d'inspiration volontaire serait absolument inactive, notre être fictif, placé toujours dans les mêmes circonstances, se trouverait réduit au sentiment fondamental absolu ou à l'état purement affectif, mais l'activité personnelle d'une part, et la sensibilité organique ou animale de l'autre, une fois mises en jeu, trouvent leur propre mobile en elles-mêmes ou dans les conditions qui leur sont respectivement inhérentes.

Les modes de l'existence sensitive et intellectuelle se lient entre eux dans une même chaîne continue ou dont les anneaux tendent toujours à se renouer ; un état premier en amène presque toujours un autre qui en diffère le moins possible ; l'exercice antérieur d'une faculté fait naître une série de produits homogènes à leur source. Si l'individu, par exemple, n'est parvenu à impressionner son organe et à se donner une faible sensation d'odeur qu'à la suite d'un effort intense et prolongé, la réflexion propre de l'acte ayant prédominé ainsi sur l'attention modale du résultat sensible, et supposant qu'il y ait ensuite un intervalle d'inaction ou de sommeil pour le centre moteur, ce sera encore par une détermination active que commencera le réveil, l'individu voudra encore plutôt le mouvement que la sensation, le moyen présent que le but éloigné ; ce sera moins le besoin de sentir que celui d'agir qui remettra en jeu les facultés, et l'exercice précédent de la réflexion appellera encore la réflexion.

Si l'attention eût prédominé dans le résultat sensible de l'acte presque inaperçu, en lui-même, soit par la faiblesse et la facilité de l'effort, soit par la vivacité que les dispositions de l'organe auraient donnée à l'impression, ce serait par une détermination sensitive propre à ce dernier que commencerait l'éveil ; l'odeur serait recherchée encore, attirée comme par une sorte d'affinité animale, et l'on pourrait assigner là un instant où le besoin, le désir iraient 
avant le vouloir ; mais dans cette hypothèse, la cause appropriée est toujours là, son influence se proportionne même à l'exaltation sensitive qui fait le besoin, et comme il n'y a point d'effort inutile, ce besoin, satisfait en naissant, n'a pas le temps encore de se transformer en désir.

Nous appellerons mémoire ou rappel la détermination active, conservée et effectuée de nouveau par le centre moteur, qui répète un effort ou un acte entièrement exécuté. Nous appellerons imagination, la détermination conservée dans l'organe sensitif ou dans le centre de réaction sympathique, qui persiste ou tend à se remettre au ton d'une impression passée.

Puisque, dans l'hypothèse actuelle, toute sensation est précédée et amenée par un acte volontaire, quoique la vivacité du résultat puisse obscurcir plus ou moins la conscience du principe, les deux déterminations précédentes devront toujours concourir ensemble, suivant des rapports, il est vrai, très inégaux ; la mémoire ne pourra être complètement séparée de l'imagination, pas plus que l'exercice de la réflexion ne l'a été d'abord de celui de l'attention. Il ne s'agit dans les deux degrés que de noter la prédominance du sentiment ou de la détermination propre de l'acte sur le sentiment et la détermination qui correspondent à son résultat modal.

Pour nous, qui distinguons ou croyons si bien distinguer nos impressions de nos souvenirs, il n'y aurait point lieu de donner ce dernier nom de souvenir à une sensation dont la cause serait actuellement présente. Mais supposez que nous ignorions absolument qu'une telle cause extérieure concourt à nous modifier ; supposez aussi que nous ayons, dans certains cas, la conscience très claire d'actes volontaires, à la suite desquels naisse infailliblement la sensation, et, dans d'autres cas, seulement la conscience vive ou obscure de cette dernière, il est certain que nous distinguerons très bien alors les modes résultant de notre activité réfléchie, de ceux qui sont passivement produits en nous, comme nous distinguons actuellement le son émis par notre organe vocal, du même son qui vient du dehors frapper l'oreille. Mais quant aux modes passifs, il n'y aurait absolument aucune raison de les séparer en deux classes de sensations et de souvenirs; car que fait la présence ou l'absence d'une cause qui n'existe pas pour nous ? Eh bien, l'individu, qui agit et sent par l'odorat seul, est, par rapport aux modes qui résultent directement de l'inspiration qu'il veut et aperçoit, à peu près, comme nous sommes par rapport aux sons articulés, dont nous avons la faculté d'impressionner volontairement notre ouïe : et nous sommes, relativement à plusieurs modifications passives et spontanées de la sensibilité, (telles que le chaud, le froid et aussi certaines odeurs qui nous poursuivent, certains tintements qui frappent nos oreilles, sans que nous puissions savoir si la cause est en nous, c'est-à-dire dans l'organe même ou hors de nous), nous sommes, dis-je, dans la même position que notre être fictif, lorsque la détermination sensitive prévaut; à cette différence près que, suivant notre hypothèse, il ne peut jamais être complètement passif dans l'odoration 
réelle, quoiqu'il puisse l'être par rapport au mobile qui la met en jeu. C'est ainsi que nous sommes encore dans les articulations, les cris, et les autres mouvements sentis, que les passions quelconques arrachent à la volonté.

Maintenant, en quoi un acte d'odoration, répété ou effectué pour la seconde ou la $n^{\mathrm{e}}$ fois, suivant une détermination motrice ou sensitive, diffère-t-il de la modification première, active ou passive, puisque la cause extérieure est toujours présente, que c'est toujours le même effort qui s'exerce avec plus ou moins d'énergie, la même impression qui vient exciter avec plus ou moins de vivacité ? Quelle raison avons-nous (surtout en nous mettant à la place de l'individu supposé, et non en le mettant à la nôtre) de distinguer sous des noms différents la même puissance, lorsqu'elle répète son acte ou lorsqu'elle l'exécute pour la première fois, son effort étant toujours suivi de succès ? S'il n'y a jamais que sensation dans divers degrés pourquoi distinguer un souvenir? Quelle est enfin la circonstance qui nous déterminera, dans l'hypothèse où nous sommes, à différencier sous les titres de mémoire et d'imagination les deux facultés fondamentales, caractérisées en premier lieu sous ceux d'aperception et d'attention? Ici l'on voit bien évidemment que si l'être moteur et sentant ne pouvait avoir, à chaque répétition du même acte volontaire, et à chaque renouvellement de l'odeur qui suit, accompagne ou prévient cet acte, le sentiment intérieur de cette répétition ou de ce renouvellement (comme tels), il agirait ou sentirait à la $n^{\mathrm{e}}$ fois comme à la première ; toute la différence que nous voulons noter ici s'anéantirait ou ne pourrait être que relative à un point de vue pris tout à fait en dehors de l'être dont il s'agit.

1. Le fondement de la différence consiste donc ici uniquement en ce que l'être, qui répète l'effort et qui éprouve une modification, se reconnaît, ou comme la même puissance qui a déjà exécuté cet acte, ou comme la vertu sentante qui a été modifiée de telle manière. Or, $1^{\circ}$ par cela seul que l'acte est répété volontairement, la puissance exécutrice se reconnaît pour la même dans la seconde détermination, et il est impossible de séparer ici le sentiment de reconnaissance ou de réminiscence de l'effort librement renouvelé, puisque ce renouvellement même le suppose. Il faut en dire autant de la modification aperçue ou réfléchie, comme résultat de l'acte répété, puisqu'elle est renfermée dans le sentiment de la même puissance ou dans le même vouloir ; d'où il suit que la mémoire ou le rappel de tout mode actif comprend essentiellement la réminiscence, qui distingue seule une seconde détermination d'une première, et que cette réminiscence n'est elle-même que la personnalité (l'aperception ou le sentiment de moi) inhérente au premier déploiement de la force sur la résistance organique, continuée dans ce déploiement effectué toujours par le même principe, et suivant les mêmes conditions, etc.

2. Quant à la vertu sentante, pour qu'elle pût reconnaître sa modification, c'est-à-dire se reconnaître pour la même, dans telle modification, il faudrait 
bien évidemment d'abord qu'elle fût constituée comme personne individuelle. Or, comment serait-elle constituée ainsi, lorsqu'elle n'est autre chose que la modification sensible? Nous avons d'ailleurs la preuve d'expérience que nos affections les plus vives, quand elles sont séparées du sentiment personnel ou des actes sur lesquels il se fonde, sont aussi comme non avenues dans notre existence successive, et que nous ne pouvons jamais savoir directement si elles sont nouvelles ou anciennes : témoins toutes nos douleurs intérieures, les degrés de froid, de chaud, de bien ou mal-être, etc. Nous pouvons donc affirmer qu'il n'y a point de réminiscence inhérente aux affections pures de la sensibilité. Dans l'hypothèse où nous sommes, si l'odeur venait seule impressionner l'organe, sans être liée à aucun sentiment de l'action nécessaire pour la produire, l'être, qui la deviendrait à chaque renouvellement, n'aurait donc aucun moyen de la reconnaître pour la même, ou ce qui est égal, de reconnaître en elle l'identité de sa propre vertu sentante. Mais ici, la sensation est toujours accompagnée d'un acte volontaire, et, quoique la conscience propre de cet acte puisse être observée indéfiniment dans le résultat sensible, la détermination n'en subsiste pas moins à un degré quelconque. De là le sentiment de moi ou l'élément personnel, qui, renfermé dans la sensation première, se retrouvera encore, dans son renouvellement effectué suivant les mêmes lois. L'individu qui exercera encore l'attention dans le résultat de l'acte, presque imperceptiblement répété, pourra donc se reconnaître, non comme la même puissance qui veut et exécute un acte, dont elle a conservé la détermination ou l'idée, mais plutôt comme être sentant modifié, dans son organisation, de la même manière dont il l'a déjà été.

La réminiscence inhérente à l'acte volontairement répété ${ }^{1}$, je l'appelle réminiscence personnelle ou réfléchie, parce que c'est par là que nous sommes

\footnotetext{
«Afin de mieux analyser la réminiscence, dit CONDILLAC, dans l'Essai sur l'Origine des connaissances humaines (chap. III), il faudrait lui donner deux noms, l'un en tant qu'elle nous a fait connaître notre être, l'autre en tant qu'elle nous fait reconnaître les sensations qui s'y répètent; car ce sont là des idées bien distinctes. » De cette observation bien approfondie et analysée jusque dans ses fondements, pouvait, je crois, ressortir un autre Traité des sensations; mais combien ne se trouve-t-elle pas effacée dans ce dernier ouvrage, où il n'est plus même question de la réminiscence, et où la mémoire se trouve caractérisée par le simple effet de l'ébranlement sensitif, qui se prolonge, après que la cause a cessé d'agir? Assurément la mémoire est bien confondue là avec la sensation ou l'affection la plus simple, et on voit s'évanouir toute distinction ultérieure sur les deux sortes de réminiscences, qui peuvent se joindre, de différentes manières, à l'effet d'un tel ébranlement organique, ou même être tout à fait séparées. Cependant, notre philosophe, en abandonnant son ancien point de vue, a bien senti la nécessité de donner à sa statue un point d'appui dans l'existence, ou de lui constituer un principe d'identité, par conséquent une réminiscence. " Ce qu'on appelle moi, dit-il dans le Traité des sensations, ne me paraît convenir qu'à un être qui remarque que dans le moment présent, il n'est plus ce qu'il a été. Tant qu'il reste le même, il n'y a point de personnalité en lui, mais dès qu'il change, il juge qu'il est le même qui a été auparavant, et il dit moi. » Assurément, pour pouvoir faire une telle remarque, il faut bien être déjà une personne constituée, un moi, et ce n'est pas d'ailleurs la statue n'existant que par les odeurs, et devenant toutes les modifications successives, qui est capable de regarder ainsi en arrière, il lui faudrait un point fixe qu'elle
} 
capables de reconnaître l'identité propre de notre être ou d'apercevoir notre existence continuée; la réminiscence, qui, s'attache seulement aux résultats de nos actes, et en apparence aux modifications passives de notre sensibilité, je l'appellerai réminiscence modale (et dans la suite objective). Ces deux espèces doivent être soigneusement distinguées, comme la réflexion et l'attention, dont elles sont dérivées ou respectivement transformées. C'est la distinction de ces deux espèces, qui sert de base unique à celle qu'on doit établir entre l'exercice de la mémoire, et celui de l'imagination; nous trouvons ainsi que tout va se rallier aux deux modes fondamentaux de notre être, très distincts, sans être séparés ; sentiment ou aperception de l'acte lui-même, prédominant sur celui du résultat ou se confondant avec ce résultat sensible qui prédomine à son tour ; conditions de cette prédominance alternative ; jusqu'ici voilà tout, et hors de là ce n'est que l'instinct qui n'est lui-même que l'absence de toute faculté. Nous verrons s'il en est de même ailleurs.

La réminiscence personnelle dans l'acte rappelé ou dans l'exercice de la mémoire, est assurée infaillible. La réminiscence modale, dans la détermination sensitive effectuée, ou l'exercice de l'imagination, est souvent incertaine, illusoire. On pourrait même caractériser cette dernière faculté par la privation complète de réminiscence qui a lieu le plus souvent dans la reproduction spontanée d'un mode quelconque ; mais il n'y aurait point alors de raison pour la distinguer de l'exercice direct de la sensibilité, du moins dans l'hypothèse où nous sommes et dans le point de vue intérieur. C'est ainsi que le vaporeux, l'homme en délire ou dans le sommeil sont bien réellement affectés, quoique nous affirmions qu'ils imaginent.

Mais pour éclaircir encore cet article important de la réminiscence modale en particulier, il faudrait faire quelque changement dans l'hypothèse simple employée jusqu'à présent. Si nous écartons, par exemple, toute cause odoriférante, l'être, qui veut se donner la sensation accoutumée, éprouve une résistance bien inattendue; il déploie de nouveaux efforts; prolonge ou

n'a pas. Au surplus, en faisant dépendre ici l'identité personnelle de la mémoire, Condillac abonde encore sur ce point dans le sens de Locke, qu'il abandonne dans tout le reste, à peu près. Mais la mémoire, telle que la définit ce dernier philosophe (Essais sur l'entendement humain, livre II) suppose bien déjà aussi l'identité personnelle ou la personnalité conservée ; en donnant l'une pour terme d'explication de l'autre, c'est expliquer le même par le même. On s'aperçoit bien ici qu'il manque un commencement; on le voit encore mieux par ce qu'ajoute Condillac dans le même article «Tant qu'un être ne change pas, il existe sans aucun retour sur lui-même, il ne peut dire moi. » À la bonne heure, mais pourrait-il le dire davantage s'il changeait toujours, si tout changeait en lui sans que rien demeurât. De plus, je demande si l'être, qui est supposé exister ainsi tout entier par un seul mode invariable, et sans aucun retour sur soi, trouvera plutôt, dans l'un des modes qu'il devient ensuite, la possibilité ou la condition nécessaire pour effectuer ce retour. Si toutes les modifications sont égales sous ce rapport, je ne vois pas comment la condition d'aperception personnelle, qui n'est dans aucune en particulier, pourrait se trouver dans le passage de l'un à l'autre. Si cette condition est inhérente à quelque modification particulière, pourquoi ne pas la distinguer des autres? 
amplifie les mouvements inspiratoires; sa réflexion prend un plus grand essor et peut-être pourra-t-il remarquer alors dans ses actes mêmes, des circonstances qui lui avaient échappé, quand son attention était absorbée dans le résultat sensible ; mais bientôt il est distrait par des affections de malaise, d'inquiétude, d'ennui, qu'il devient sans pouvoir s'en rendre compte ; la sensibilité, portée par l'habitude à un certain ton, réclame ses aliments propres, des besoins inconnus se font sentir, ils déterminent des mouvements brusques, précipités et d'une énergie supérieure à ceux qui étaient accompagnés auparavant de réflexion et de volonté ; cette dernière force est opprimée, ce n'est plus la même qui dirigeait et commandait; si le moi peut s'apercevoir ou se retrouver dans ce tumulte des affections, il sent bien qu'il est mal, qu'il n'est pas comme il a été, comme il voudrait être, il sent son impuissance et par suite sa dépendance, il sent le besoin, il est bien près de sentir de véritables désirs.

Dans cet état, si nous substituons une odeur différente de la première et qui soit encore plus faible, ou qui demande un effort d'inspiration plus marqué et plus soutenu, cette odeur sera sentie dans le rapport à l'action forte, que le besoin détermine, et, quoique la sensibilité ne soit point entièrement satisfaite, l'état de malaise qui a précédé, la nouveauté de l'impression qui compense sa faiblesse relative, y attacheront de nouveau toute l'attention, la volonté sera déterminée, hors du besoin même, à soutenir le mouvement inspiratoire dans le degré nécessaire pour conserver la sensation, la rappeler quand elle s'échappe, ce qui demandera alors un effort plus pénible, et pourra contribuer à développer la réflexion.

Dans ce passage d'une modification ou d'une odeur particulière à une autre, qui en diffère par le caractère sensitif et le degré d'effort plus intense qu'elle nécessite, on pourrait croire qu'il y a une comparaison faite entre les deux modes affectifs, dont l'un se reproduit par la mémoire, dans le même instant que l'autre est présent au sens externe, ce qui fait reconnaître l'ancienneté de l'une, la nouveauté de l'autre, et fonderait ainsi la réminiscence, et par elle, l'identité personnelle. Mais nous pouvons observer, et notre expérience confirme assez, que la réminiscence modale s'exerce très peu sur les odeurs elles-mêmes ; la comparaison et la distinction de ces modes se fondant en nous sur plusieurs perceptions ou circonstances simultanément associées ${ }^{1}$. Dans l'hypothèse actuelle, pendant que l'attention s'applique à la sensation nouvelle, la réflexion peut s'exercer dans le déploiement plus énergique de l'effort nécessaire pour la produire ; c'est toujours la même puissance qui veut et qui agit, mais suivant une détermination différente de celle dont elle avait contracté l'habitude. Voilà la base réelle de la comparaison et de cette réminiscence qui se réfléchit, de deux actes identiques dans leur cause, sur les

Sous ce rapport, Bonnet et Condillac ne pouvaient pas plus mal choisir leur point de départ, pour distinguer dans la sensation l'origine de toutes les facultés. 
modes divers résultants qui leur correspondent, et part ainsi de la constance même pour apprécier et mesurer les changements.

Si chaque odeur dépendait ainsi d'un mouvement distinct approprié, il me semble que les modifications de cette espèce se distingueraient entre elles dans leur succession ou leur ensemble deviendraient la matière de comparaisons réfléchies, et seraient enfin dans notre mémoire, à peu près comme d'autres perceptions dont nous parlerons. Mais ces impressions se lient à des mouvements instinctifs continuels, et presque toujours inaperçus, et (sans parler de la manière dont les molécules odorantes affectent, comme par diffusion, les extrémités nerveuses et quelquefois tout le système sensible), la même inspiration volontaire est accompagnée ou suivie des sensations les plus opposées. Il ne saurait donc y avoir de distinction ou de rappel des odeurs comme des sons, par autant de mouvements déterminés avec intention, avec un effort réflectible.

Cette circonstance même de la passivité, réellement inhérente aux odeurs, nous fera concevoir comment l'être fictif, qui a commencé à déployer son activité sur de tels modes, et qui, dans la situation hypothétique où nous l'avons placé, se serait accoutumé à les sentir sous un rapport de subordination nécessaire aux mouvements dont il dispose ${ }^{1}$, comment, dis-je, cet être tiré de cette situation forcée, et rendu à l'ordre commun des choses, pourrait partir de la réflexion pour entrer dans une sorte de monde extérieur invisible imaginaire où il est attiré par l'exercice de facultés d'une autre espèce.

\section{II. - Nouvel ordre de facultés passives.}

\section{Retour à la table des matières}

En écartant et rapprochant tour à tour de l'odorat différentes causes d'impressions, qui agissent toujours assez faiblement, ou à une distance assez grande, pour exiger un effort inspiratoire plus ou moins marqué, nous donnerions un plus grand développement aux facultés précédentes.

L'individu, qui se sent toujours une puissance identique, invariable et constante, déployée sur un terme organique, diversement modifié, devra perdre, après un certain nombre d'expériences, l'habitude, qu'il avait contractée d'abord, de s'attribuer les sensations comme les mouvements, et de vouloir les unes comme les autres. L'action se sépare pour lui du résultat sensitif; il veut

C'est l'inverse de l'hypothèse de Condillac et Bonnet, et en cela plus conforme à la réalité des choses, mais non point à la génération des facultés qu'ils avaient en vue. 
toujours celle-là, il sent qu'il en dispose, mais il est incertain de l'autre. Si nous avons tenu pendant un certain temps la même odeur à sa portée, puis, si nous l'avons écartée, pour la lui rendre encore, il aura plus ou moins d'assurance de la reproduire, suivant que son effort aura été plus ou moins souvent suivi de succès, ou inutile. Il peut exister pour lui déjà des chances, des probabilités, qui motivent ses craintes, ses espérances, il sent qu'il est dépendant ou subordonné dans ses sensations, libre dans ses actes, et il commence à soupçonner l'existence d'une cause invisible, qui a, pour le modifier, un pouvoir égal ou supérieur à celui qu'il retrouve en lui-même dans ses actes libres. C'est là qu'il faut rapporter l'origine du phénomène que nous appelons croyance, et non point aux perceptions directes du toucher, de la résistance, etc.

Entourons-le, maintenant, de diverses odeurs fortes ou fâcheuses qui n'attendent pas l'effort inspiratoire pour pénétrer l'organe ; ici, sa dépendance n'est plus douteuse, il sent sa passivité complète parce qu'il a le souvenir d'un état d'activité, dont nous pouvons même lui laisser, par alternatives, le sentiment immédiat. Il se reconnaît alors comme une vertu sentante, comme une puissance inactive ou dégradée, parce qu'il a été auparavant et qu'il se reconnaît encore une puissance.

Quand son attention est tout entière à une odeur agréable, et que tous ses efforts tendent à la retenir une force invisible la lui enlève subitement et la remplace par une odeur repoussante, cet agent mystérieux quel qu'il soit, qui le poursuit, quand il veut le fuir, lui échappe quand il le cherche, lui résiste quand il l'appelle, est différent de son moi ; c'est une puissance comme lui, mais plus forte que lui, d'où dépend tout son bonheur ou son malheur. C'est elle qui sera désormais l'objet de ses désirs, c'est en elle qu'il espère, c'est elle surtout qu'il craint, il lui portera des vœux, lui dressera des autels.

Une imagination naturellement superstitieuse ${ }^{1}$ dirigerait donc les premiers pas que ferait, pour sortir de lui-même, l'être sensible et moteur, qui sent sa dépendance, non pas avant, mais aussitôt qu'il a conscience de sa causalité, c'est-à-dire l'aperception de son existence. La résistance au désir doit amener, non pas la connaissance, mais la croyance de quelque chose qui existe hors de l'être sentant, non pas la perception, mais la persuasion d'un non-moi. Ce premier pas est nécessaire, il est vrai, mais il ne l'est que lorsque l'on part d'une activité réfléchie qui se prend elle-même pour terme de comparaison, et non

Diderot, dans une de ces saillies philosophiques qui lui étaient si communes, et qui l'ont quelquefois si bien servi, personnifie aussi chaque sens séparé et lui assigne un certain caractère moral qui le distingue. C'est le palais qui est le superstitieux, et l'odorat le voluptueux. Mais le premier s'applique immédiatement à son objet, il pourrait donc bien être taxé de matérialisme comme le toucher. Le deuxième, au contraire, devine ou imagine son objet comme dans une sorte de vague. La volupté comme la superstition est amie du mystère. Aussi vont-elles souvent ensemble. 
point quand on part des affections simples, qui ne se comparent ni ne se pensent toutes seules.

Je ne pousserai pas plus loin une hypothèse, dont l'invraisemblance aura déjà peut-être trop révolté mes juges. Elle rappelle d'ailleurs celle de deux grands maîtres avec qui je ne prétends sûrement pas soutenir le parallèle, en entrant dans cet ordre de modifications et de facultés qui se rapportent entièrement à la sensibilité, subordonnée elle-même à l'action des objets. Je reconnais la supériorité de la plupart de leurs analyses, et je ne tenterai pas d'y rien ajouter, en suivant encore une méthode hypothétique que je crois bien insuffisante, et dont je ne tirerais pas assurément le même parti qu'eux. Mais c'est le point de départ qu'il m'a paru très important de fixer.

En admettant avec ces maîtres qu'il pouvait y avoir des facultés ou opérations intellectuelles exercées, développées hors de la connaissance du monde extérieur ${ }^{1}$, et des idées acquises hors de toute représentation objective, je n'ai pas cru que ces facultés dussent se rapporter à une seule et même condition, ni que ces idées ressortissent uniquement des modifications passives occasionnées par ces objets inconnus. En donnant hypothétiquement l'initiative à l'action que la volonté exerce plus ou moins sur tous les sens externes, et dont les analystes n'ont tenu aucun compte, j'ai voulu signaler un élément essentiel trop négligé, qui, entrant dans tous les produits de l'intelligence humaine, doit changer les caractères conventionnels, sous lesquels on nous représentait nos facultés, et les rendre plus conformes au modèle que la réflexion nous découvre.

J'observerai en finissant, et pour justifier le point de départ que j'ai choisi :

1. Que l'être sentant et moteur, une fois livré aux excitations passives, et multipliées des causes dont nous l'entourons, affecté, impressionné incessamment sans agir ou sans s'apercevoir qu'il agit, perdrait l'usage de ses

C'est ce qu'avait nié en premier lieu un philosophe qui a profondément creusé la mine ouverte par Condillac. Voyez les Mémoires et l'Idéologie de M. DE TRACY.) Ce philosophe pensait alors, que hors de l'exercice de la motilité, c'est-à-dire de la faculté de faire des mouvements et d'en avoir conscience, il ne pourrait y avoir lieu à l'action du jugement, ni par conséquent à aucune des facultés intellectuelles dont il est la base. Le mode, qu'il introduisit le premier dans l'analyse, sous le nom de sensation de mouvement, ne fut envisagé dès lors par lui que dans la locomotion du corps en masse, ou dans celle de l'organe propre du toucher, et le mouvement contraint par un obstacle lui parut renfermer la condition première de la connaissance de corps résistant, du jugement, et par suite de tout le reste. En partant du même principe de motilité, je l'ai suivi depuis, de mon côté, dans l'exercice de chaque sens en particulier, et je me suis convaincu que là où s'applique la faculté du mouvement volontaire, là seulement est la connaissance, la perception. J'ai substitué la résistance ou l'inertie organique à la résistance étrangère, et j'ai vu lu facultés originairement constituées, non pas exclusivement dans ce mouvement contraint qui nous apprend qu'il existe quelque chose hors de nous, mais plus généralement dans l'effort qui est essentiellement relatif à quelque terme, qu'il s'applique soit au corps propre, soit au corps étranger. 
facultés que nous avons vu naître en lui, plutôt qu'il ne trouverait les moyens de leur développement, au sein de ces affections tumultueuses, de ces besoins impérieux, de ces désirs d'autant plus exaltés qu'ils sont plus vagues, plus indéterminés dam leur objet. L'attention ne suit plus la direction de la volonté, ce n'est plus une puissance proportionnée à la vivacité de l'impression, elle n'est plus que la sensation même (dont il serait en effet bien inutile de la distinguer par un terme indicateur d'un acte, qui n'existe point dans cette hypothèse). La mémoire n'est que le résultat d'un ébranlement organique ; l'imagination, qui n'en diffère pas, n'est encore que la sensation continuée, affaiblie ou avivée, suivant les dispositions de l'organe externe, ou de ceux de la sensibilité intérieure avec lesquels il correspond.

Dans l'hypothèse d'un être qui deviendrait successivement toutes les odeurs qui l'affectent, soit par les sens, soit par l'imagination, ou la mémoire, et dont toutes les facultés, nominalement distinguées, se réduisent bien complètement en effet à la sensation, comment peut-il y avoir quelques idées abstraites ou possibilité d'effectuer, ainsi que le dit Bonnet, des abstractions modales? Les odeurs, comme toutes les autres modifications affectives, se confondent entre elles dans le sens et sont aussi irrévocables dans le souvenir; c'est un fait même pour nous qui avons plusieurs moyens de les distinguer. Comment donc pourront-elles s'abstraire d'un composé, qui affecte toujours comme simple?

Comment retrouver dans une succession de modes variables, les idées d'unité et par conséquent de pluralité, celles d'identité, de durée, de temps, etc. ?

Ces idées sont-elles encore des sensations, ou abstraites indifféremment de toute sensation quelconque ? Cette question me paraît importante, et j'en ferai l'objet de ma dernière observation.

2. L'être fictif, tel que nous l'avons supposé d'abord, ne peut s'apercevoir ou être constitué moi dans l'action qu'il s'exerce et la modification première qui en résulte, sans avoir l'idée ou le sentiment de son unicité. Tant que la même sensation suit ou accompagne immédiatement son effort, il est un dans le produit ou l'effet comme dans la puissance ou la force. En disant d'abord moi il dit un ; en répétant la même action suivie du même résultat, il dit encore un, ainsi de suite. Le temps est né pour lui, c'est, comme disent les Kantistes, la forme pure sous laquelle il s'aperçoit et se retrouve toujours le même. L'identité est donc encore inhérente à l'aperception personnelle ou au mode actif constant qui lui sert de base, et avant tout (ce qu'ont négligé d'observer les métaphysiciens qui ont marché sur les traces de Descartes, de Leibnitz, de Kant) aux conditions d'où dépend la reproduction constante de ce mode. Lorsque, par les nouvelles circonstances où nous plaçons l'individu, les sensations varient et se succèdent, pendant que l'effort reste le même, il y a pluralité sentie ou perçue dans l'unité, il peut y avoir aussi une durée mesurée 
par l'intervalle des modifications accidentelles successives, parce qu'il y a un module fixe, un terme commun auquel tous les instants se rapportent.

De telles idées ne sont point abstraites, pour l'être que nous avons supposé ; ce sont, au contraire, les premiers et les plus simples résultats de ses facultés originelles, inséparables du sentiment qu'il a de son existence active. Ces modes sont pour lui ce que sont, ou paraissent être pour nous, les impressions passives qui nous affectent. Ce sont les idées simples de la réflexion, et non point des abstractions réfléchies. Ces idées que nous appelons abstraites, celles de moi, d'unité, d'identité, de temps, etc., et celles des opérations ou formes propres sous lesquelles l'être actif et intelligent s'aperçoit lui-même, constitueraient donc le fond primitif et naturel des connaissances de l'individu, dont l'activité motrice aurait pris un assez grand développement, avant que les impressions, trop affectives et trop multipliées, n'eussent assailli ses sens encore fermés en partie.

Lorsque la sensibilité et l'imagination sont en plein exercice, et qu'une attention commandée ne marche plus qu'à leur suite, celle-ci ne s'attache plus qu'aux modes résultant d'un effort trop facile. Lorsqu'ils portent en eux-mêmes les conditions naturelles de leur distinction dans le sens, de leur révocabilité dans le souvenir, elle les circonscrit, les sépare, en forme de nouveaux groupes, Voilà nos idées abstraites modales objectives. Alors aussi la réflexion demande un grand effort; les idées simples, qui en sont les produits immédiats, ne sont plus que des résultats éloignés d'autres opérations confondues avec les sensations et les images dont elles paraissent faire partie ; on travaille souvent en vain à les séparer, et les produits, que l'on obtient par cette décomposition artificielle, s'appellent abstractions réfléchies, lorsqu'elles ne sont pas purement logiques. 


\section{Chapitre III}

\section{Des fonctions de l'ouïe et de la voix, des facultés et des idées élémentaires qui peuvent en être dérivées ${ }^{1}$}

\section{$\underline{\text { Retour à la table des matières }}$}

Les impressions immédiates que fait sur l'organe de l'ouïe un fluide extérieur en mouvement, l'ébranlement et l'espèce de commotion affective que ce fluide sonore a la propriété de communiquer à tout le système sensible, sont bien loin sans doute de ces caractères éminents de relation, d'activité, de conscience, de netteté ou de distinction dans le sens, de révocabilité dans le souvenir, que doivent acquérir les perceptions auditives dans la suite des progrès d'une vie intellectuelle, dont elles deviennent le premier mobile et constituent presque tout le fondement.

1 Le rapport particulier, sous lequel j'envisage les fonctions des sens, me dispense de faire l'analyse de celle du goût. J'aurais pu de même omettre celle de l'odorat, si je ne me fusse occupé que de l'ordre réel et naturel de la dérivation de nos facultés intellectuelles des sens où elles prennent véritablement leur origine; mais j'avais un autre but, qui n'a pu échapper à mes juges, et qu'ils pourront encore mieux apprécier, après avoir entièrement parcouru cette analyse. Je crains de n'avoir que trop insisté sur une pure hypothèse, et je ne la renouvellerai point en cherchant dorénavant dans l'exercice d'un sens ce qui n'y serait point actuellement compris, ou ne pourrait en être dérivé, qu'en forçant toutes les suppositions. Les fonctions propres de l'odorat et du goût se rapportent directement à la vie intérieure ou nutritive; elles sont en pleine activité, sous la loi exclusive même de l'instinct. Ce fait, sur lequel on ne peut élever aucun doute, a déterminé l'auteur d'une nouvelle division physiologique (voyez l'ouvrage intitulé : Division des phénomènes physiologiques, par $\mathrm{M}$. DUBUISSON, an XI) (qui me paraît avoir mieux précisé dans certains points, les vues du célèbre Bichat) à ranger ces deux sens dans une classe séparée de celle qui comprend les organes ou instruments de la vie active de conscience. Le même point de vue se trouve indiqué dans un Mémoire sur l'habitude, couronné en l'an X par l'Institut national.

Tant que les affections et les mouvements de ces organes s'accomplissent ainsi directement ou par réaction sympathique, ils ne peuvent en aucune manière servir d'origine aux facultés intellectuelles; c'est lorsqu'ils passent ultérieurement et en partie sous la puissance du vouloir, qu'on peut y trouver quelque lumière, ou y assigner quelque moyen de développement. Considéré sous ce dernier rapport et dans la manière dont il est expressément activé et immédiatement appliqué à son objet résistant ou solide, le sens du goût pourrait être assimilé, jusqu'à un certain point, au toucher ; il contribuerait ainsi avec lui à la connaissance directe des existences étrangères, et au développement des facultés originelles qui s'y rapportent. Ce que nous avons à dire, sur ce dernier article, suppléera donc à l'omission actuelle. Quant à ce qui concerne les fonctions spéciales du goût, et leur rapport à l'instinct de nutrition, nous n'avons pas besoin d'en parler. 


\section{I. - Fonctions passives de l'ouïe.}

Retour à la table des matières

L'ouïe, plus que l'odorat, est immédiatement accessible aux impressions qui viennent, pour ainsi dire, la chercher sans qu'elle paraisse douée d'aucune force de locomotion pour aller au-devant d'elle, d'aucune activité propre pour les appeler ou les écarter, les modérer ou les suspendre. Ce n'est point d'abord le nerf auditif ou le centre, dans lequel la perception est censée directement s'accomplir, qui s'y trouvent seuls intéressés, mais encore diverses parties de la même combinaison organique. L'enfant, dès sa naissance, trémousse au bruit qu'il n'entend point encore; dans l'état de surdité complète, comme dans une audition toute passive, l'affection sonore porte sur différents sièges, tantôt comme chatouillement interne, tantôt comme une véritable douleur, et plus le physique et le matériel du son renferme ainsi les conditions d'une excitabilité sensitive, plus il s'éloigne de celles de la connaissance et de l'aperceptibilité personnelle qui en est la base. Par cela seul donc qu'un être sentant deviendrait toutes les affections de cette espèce, il serait en dehors des lois et des moyens originaires de l'intelligence, et ne pourrait s'y élever de ce point par aucune transformation.

L'hypothèse précédente sur l'odoration active a pu nous. conduire à admettre dans le mode relatif d'effort inspiratoire (indépendant, de toute impression provocante du dehors, et, même de tout résultat sensible) un sujet constitué comme force agissante sur ou par un terme organique. Lorsque l'impression d'odeur est venue ensuite se joindre, pour ainsi dire, à ce fond d'existence aperçue, soit comme résultat d'action, soit passivement, nous avons pu retrouver là les deux éléments d'une véritable sensation composée et le principe générateur de deux ordres de facultés et d'idées élémentaires.

Ici, il n'y a pas lieu à feindre même aucune hypothèse de cette nature. L'ouïe ne se mettra point en jeu, d'elle-même, hors d'une cause extérieure d'impression, et lorsque celle-ci arrivera à l'organe, l'affection sera nécessairement pure, et pas même composée de ce jugement, qui constitue pour l'individu un siège, auquel il rapporte des modifications, qu'alors seulement il peut être dit sentir sans les devenir.

Sans doute il y a dans le développement actuel de nos facultés une puissance d'audition bien plus marquée, et qui a bien de tous autres effets que celle d'odoration. La force motrice, ou la volonté présente dans l'état de veille 
à tous les organes qu'elle tient sous sa dépendance immédiate, active, suivant les cas, leurs divers phénomènes par une détermination expresse. Les perceptions auditives semblent bien être souvent des produits partiels de cette activité, expressément déployée sur le sens, pendant que la cause sonore l'impressionne du dehors. Les muscles de l'oreille mis en jeu par une fonction que l'on peut, ce me semble, appeler proprement volontaire, sont portés de cet état commun de tension, qui fait la veille particulière de l'organe, à un ton supérieur qui détermine l'audition distincte. Et c'est cette intervention positive de la volonté que le langage exprime par le terme écouter, dont le sens n'est, pour personne, le même que celui d'entendre (comme l'acception du mot flairer diffère de celle du mot sentir, appliqué particulièrement aux odeurs ${ }^{1}$.

Mais ici l'application de la volonté est-elle première, et aussi directe qu'elle le paraît? A-t-elle pu, dans l'origine, peut-elle même actuellement prendre quelque initiative et une prédominance réelle sur les impressions qui la provoquent? Ne leur est-elle pas au contraire plus ou moins subordonnée ? Ce qu'il y a de certain, c'est que, hors de ces impressions, il n'y a point de sentiment intérieur d'un effort ou acte quelconque, qui ait pour terme l'organe de l'ouïe considéré dans ses fonctions propres. Et ce n'est point seulement parce que l'action motrice s'accomplit ici intérieurement ou hors de la portée d'un autre sens qui pourrait en objectiver le résultat, puisqu'il y a d'autres mouvements qui s'exécutent d'une manière tout aussi secrète, et dont nous n'apercevons pas moins clairement, dont nous ne nous approprions pas moins l'entière exécution. Mais c'est que la force motrice ne suit point ici sa propre détermination, c'est qu'elle agit toujours d'une manière subordonnée, c'est qu'elle manque des moyens propres à lui réfléchir ses produits distinctement de toute autre impression et lui apprendre ainsi à les diriger.

L'effort auditif n'ayant donc jamais pu être aperçu ni déployé originairement, hors de la sensation présente, ne formera point avec celle-ci un composé réel. L'acte ou le mouvement ignoré par le sujet est comme nul pour lui, ainsi que l'est la cause externe d'impression pour l'être moteur et sensible qui, méconnaissant son influence, s'en attribuerait tout l'effet. De même que l'individu odorant comme il veut, et parce qu'il veut, est pleinement actif pour lui-même, quoique impressionné du dehors à son insu, celui qui entend ou sent

\footnotetext{
Les verbes sont, il est vrai, également actifs dans les deux cas, et comme dit Bayle dans l'article déjà cité à l'occasion de la girouette, nous nous servons du verbe actif pour désigner des modes dont l'âme (ou le moi) n'est pas la cause efficiente, comme nous nous en servons quant aux actes de la volonté. Ainsi nous disons (je souffre, je sens de la douleur, je vois, etc.) comme je veux, j'agis, je regarde, etc. Toute proposition ou tout jugement énoncé suppose bien réellement une participation de l'être qui sent les modifications qu'il éprouve et aperçoit celles qu'il concourt à produire par un acte exprès de la volonté. Dans ce dernier cas, l'activité proprement dite existe dans la réalité comme dans l'expression. Dans le premier, la phrase, simplement énonciative d'abord, s'est contractée en un seul terme qui porte la livrée des verbes d'action, mais sans en avoir, pour ainsi dire, le corps ou la substance. Nous reviendrons ailleurs sur cet objet.
} 
simplement les sons extérieurs, devra se sentir (lorsqu'il est constitué moi) comme s'il était entièrement passif dans l'acte inaperçu qui concourt avec la sensation, ou la détermine peut-être. C'est ainsi que les mêmes faits jugés au dehors par l'observateur, ou pris intérieurement dans la conscience du sujet, sont le plus souvent en opposition absolue. (Voyez le chapitre précédent.)

Dans l'état actuel, on pourrait caractériser et circonscrire hypothétiquement les facultés intellectuelles d'un être supposé borné au sens passif de l'ouïe, et l'on ne retrouverait en effet que des caractères ou des circonstances de la même sensation ; rien ne pourrait être pris, pour ainsi dire, qu'en dedans d'elle. L'être fictif serait dit donner son attention, rappeler, comparer, etc., les sons qu'il deviendrait tour à tour, et ces opérations qu'il exerce, il ne les veut, ne les connaît, ni n'a aucun moyen de les connaître ou de se connaître lui-même.

Cependant, le sens de l'ouïe est éminemment réfléchi : c'est lui qui entend tout, répète, redit tout, jusqu'aux modifications les plus intimes ; c'est le sens de la conscience, c'est celui de l'entendement, de cette faculté par excellence, par laquelle l'être pensant entend (dans toute la force du mot) à tous les actes qu'il détermine.

Il est de fait que la privation de ce seul sons entraîne celle de presque toute l'intelligence. Sans lui, l'individu abandonné à lui-même, demeure dans un état voisin de l'imbécillité, et lorsque le bienfait inestimable d'une éducation soignée, dirigée par le génie, parvient à l'élever à la dignité de l'être pensant, qui conçoit les choses en s'en faisant des images claires, exactes et distinctes, jamais il ne le porte à ce degré où il puisse concevoir ses propres actes, s'en faire des idées, se réfléchir, et se penser lui-même. Tous les autres sens suppléent l'ouïe dans ce qui se représente, aucun ne peut la remplacer dans ce qui s'aperçoit et s'entend au dedans de nous.

D'où lui vient donc ce caractère de réflexion et d'activité qui semble d'abord si étranger à ses fonctions directes ? Faut-il l'attribuer uniquement à la faculté de recevoir les impressions du fluide sonore, et d'en être affecté ? N'y a-t-il pas un organe de communication, un mobile propre d'activité qui, n'étant d'abord qu'une dépendance essentielle du sens immédiat de l'ouïe, reprend bientôt sur lui l'initiative réelle d'action, lui transmet, lui réfléchit celle de la volonté qu'il reçoit plus directement, le fait rentrer dans son domaine, et le constitue ainsi le premier organe de l'intelligence? 


\section{II. - Fonctions actives vocales. Origine naturelle de la réflexion et du jugement.}

\section{$\underline{\text { Retour à la table des matières }}$}

On ne voit point d'êtres de notre espèce, doués de la faculté d'entendre, et privés de celle de former eux-mêmes des sons, ou d'imiter ceux qui les frappent, et la nature semble avoir pris soin de modeler, l'un sur l'autre, les organes de l'ouïe et de la voix, en proportionnant presque toujours la finesse ou la grossièreté de l'un, à la flexibilité ou à la rudesse de l'autre. Cependant, on a trouvé quelques individus, dans l'état sauvage, dont l'instrument vocal s'était, pour ainsi dire, paralysé par un défaut absolu d'exercice, et qui, quoique frappés par le bruit, paraissaient totalement privés de la faculté de distinguer des sons ordinaires, ou n'y donnaient absolument aucune attention; ce qui prouverait déjà que la perceptibilité auditive se rapporte bien plus à l'organe actif, qui répète intérieurement et imite d'abord, comme par une sorte d'instinct moteur sympathique, les sons qui viennent frapper l'ouïe, qu'au sens passif qui reçoit les impressions immédiates.

Nous trouvons donc ici une fonction motrice particulière, qui fait bien plus que remplacer celle dont nous avons précédemment observé ou plutôt supposé les produits. Là comme ici, cette fonction concourait à rendre perceptible l'impression faite par une cause étrangère, à l'approprier à la conscience de l'individu, comme une de ses créations. Mais ce terme de motilité étant confondu avec l'organe sensitif, impressionnable par une cause extérieure présente, quoique ignorée, la sensation qui en serait l'effet réel suivrait nécessairement l'effort inspiratoire, sans qu'il y eût aucun moyen de séparer les deux produits élémentaires, sans que la volonté pût reconnaître le sien. De là l'incertitude, l'hésitation, lorsque les choses viennent à changer autour de l'individu, la difficulté de reconnaître ce qu'il y a de réel d'une part, et d'illusoire de l'autre, dans l'exercice de la même puissance, l'impossibilité de distinguer ce qui se sent de ce qui s'imagine, et souvent ce qui se réfléchit en soi comme création de l'effort, de ce qui se représente, pour ainsi dire, dans un mode forcé venu d'ailleurs. De là enfin, ce vague imaginaire qui s'attache (dans la résistance au désir) à la conception d'un non-moi.

Ici l'organe moteur est séparé de celui qui reçoit l'impression, et n'en correspond pas moins avec lui, n'en active pas moins, n'en active même que mieux ses fonctions perceptives. À l'instant où l'ébranlement sonore est 
communiqué à l'ouïe, outre la réaction motrice simultanée et inaperçue qui complète la sensation, il y a de plus une détermination du même ordre, qui va mettre en jeu l'instrument vocal ; celui-ci répète le son extérieur et lui fait écho, l'ouïe est frappée de deux impressions, l'une directe, l'autre réfléchie, intérieure ; ce sont deux empreintes qui s'ajoutent, ou plutôt c'est la même qui se redouble, c'est comme si l'odorat, étant d'abord directement impressionné, l'effort inspiratoire qui s'exercerait par un autre organe correspondant (tel que la bouche, par exemple), avait la faculté de reproduire absolument la même sensation, celle-ci se trouverait véritablement redoublée, savoir : une première fois par l'action de la cause extérieure accompagnée ou non de quelque degré d'effort inaperçu et une seconde fois, sans le concours d'aucune cause autre que la volonté, avec un effort perceptible dans sa détermination ou dans son produit sensitif; je crois que le parallèle est exact.

Maintenant, pendant que l'impression se fait sur l'organe externe, nous concevons comment la détermination vocale, qui s'y joint, peut la renforcer en la doublant, l'active et lui communique le caractère d'aperception inhérent à l'effort volontaire. La cause extérieure cessant d'agir, la même détermination peut s'effectuer encore, et l'organe sera impressionné de nouveau de dedans au dehors, la sensation sera complète, sans qu'aucune force étrangère concoure à la produire. C'est ici une circonstance unique et qui devait bien frapper, ce semble, les philosophes qui ont fait l'analyse des sens, pour connaître la part que chacun d'eux peut prendre à nos facultés originelles.

Dans l'odoration active, telle que nous l'avons supposée, l'individu attribue bien d'abord à sa puissance les premières modifications qu'il reçoit, mais il est dans une illusion dont le spectateur juge, et que l'expérience ou le cours naturel des choses doit promptement dissiper. C'est seulement lorsque ses impressions deviennent tout à fait passives, ou qu'il se sent modifié malgré lui, qu'il soupçonne et imagine vaguement quelque force différente de la sienne.

Dans l'effort vocal et la sensation qui le suit ou l'accompagne, il n'y a point d'illusion que l'expérience réfléchie doive détruire ; l'ouïe est, en quelque sorte, elle-même le juge de la différence des deux impressions directe externe, et réfléchie intérieure qui se succèdent. Celle-ci se rattache invariablement à la cause moi qui la produit avec conscience. L'autre se rapportera donc, par une simple perception de contraste, à quelque autre cause non moi, conçue alors seulement sous cette propriété négative. Il n'y a pas là de surprise, point de ces grands effets d'imagination, que peut faire naître la résistance au désir, quand il est, excité par toute la force du besoin; les sons n'ont point cette propriété excitative qui convertit d'autres sensations en besoins impérieux.

Dès que le son direct se distingue du son produit et réfléchi par un contraste marqué, qui se fonde d'une part sur l'activité de l'effort dans une modification, et sur son absence dans l'autre, le souvenir ne peut se confondre avec 
l'impression, pas plus que la perception avec la sensation passive ; ce souvenir n'est ici qu'une perception réelle, qui frappe encore le sens, quoiqu'il n'y ait plus de cause extérieure, et la mémoire des sons n'est autre que la faculté qu'a la voix d'impressionner en partie l'ouie, comme le ferait l'objet sonore luimême, s'il était encore présent; je dis en partie parce qu'il y a effectivement deux parties dans l'impression que reçoit l'oreille : l'une, purement sensitive, reste bornée à l'organe qu'elle affecte, ou s'étend par consensus à tout le système sensible, suivant la nature du son, et en particulier la qualité du timbre : l'autre partie, spécialement appropriée à la voix, s'adresse presque tout entière à son organe, c'est celui-ci qui la reçoit, l'entend pour ainsi dire, la répète et l'imite dans sa dernière perfection.

Ces deux parties (audible ou vocale) ne se trouvent pas également réunies dans chaque son ou chaque suite de sons, et les habitudes, ou certaines dispositions propres du sujet, peuvent faire quelquefois prédominer l'une sur l'autre. La partie audible du son direct est une simple sensation ; l'individu qui y serait borné ne percevrait, ne distinguerait rien par l'ouïe, il serait frappé ou ébranlé par ces impressions comme par du bruit. C'est de cette manière que serait probablement modifié (par des sons quelconques) celui qui naîtrait avec l'instrument vocal paralysé, quoique l'organe auditif fût en bon état. C'est ainsi que nous le sommes nous-mêmes, lorsque les sons ont certains caractères trop affectifs ou qu'ils sont dénués de toute analogie avec ceux que nous pouvons rendre et imiter par les accents de la voix; de telles impressions n'entrent point ou ne persistent point dans la mémoire.

La partie vocale réunit tous les caractères de la perception ; l'organe qui la reçoit, sans en être sensiblement affecté, ne fait que la transmettre à un centre d'activité, qui la complète dans son siège direct comme sensation, l'effectue de nouveau, et la recueille encore dans son redoublement, comme produit d'un effort qu'il détermine : à chaque impression reçue correspond un mouvement particulier, et une détermination du même ordre qui prépare le rappel volontaire de la même sensation, aussi distincte quand elle part du dedans que lorsqu'elle venait du dehors. 


\section{III. - Résultats des deux fonctions. Facultés passives et actives.}

$\underline{\text { Retour à la table des matières }}$

Suivant que tels sons seront ou directement sentis comme impressions simples auditives, ou perçus par redoublement dans leur partie vocale, les effets subséquents auront donc entre eux des différences considérables; ce seront de tout autres opérations ou facultés qui ressortiront de cette origine, une en apparence, double en réalité.

Et d'abord, quand l'impression se fait du dehors sur l'ouïe, si le caractère affectif est seul ou très prédominant, la sensation auditive pourra bien être nommée attention, lorsqu'elle devient exclusive de toute autre, fût-elle même jusqu'au point d'absorber le sentiment propre de l'existence personnelle ; mais il est bien évident que cette faculté nominale qui suit la force des impressions et s'y proportionne, n'est qu'une circonstance particulière de la même propriété affective, et non point une puissance ni un mode d'exercice quelconque de celle que nous sentons en nous-mêmes; aussi ne contribue-t-elle en rien à distinguer les modifications ni à les approprier au rappel. Lors même qu'il se joint à l'impression directe une détermination vocale qui active, qui jusqu'à un certain point, l'effet sensitif et lui communique quelque caractère de perceptibilité, il suffit encore que la partie affective domine à un assez haut degré, et qu'une attention très subordonnée s'attache exclusivement au produit composé de l'effort faible qui vient de l'individu, et de l'impression forte qui vient du dehors, pour que les sons demeurent encore plus ou moins confus dans le sens, et par suite peu révocables dans le souvenir.

A. Imagination. - La détermination sensitive, résultat nécessaire de ce premier effet peut persister dans l'organe ou le centre cérébral : de là, une sensation affaiblie qui se continue, ou se reproduit dans l'absence de la cause extérieure, sensation réelle qu'un observateur mieux informé pourrait caractériser d'imagination, mais qui, n'étant accompagnée, dans celui qui l'éprouve, d'aucun acte de réminiscence, d'aucune idée ou sentiment de causalité, n'a rien qui la distingue de l'impression directe la plus simple.

B. Concours de l'attention et de la réflexion. - Mais lorsque le son est de telle nature qu'il s'approprie principalement à la fonction vocale, la perception première se fait d'une tout autre manière, et les résultats sont aussi bien différents. 
Pendant que l'ouïe est frappée et que la voix répond ou tend à répondre, en se portant activement au même unisson, l'attention, qui marche à la suite de la volonté et n'est plus commandée par la vivacité de l'impression auditive, peut se fixer encore sur cette dernière, et accroître même, comme distinguer et éclaircir ses produits ; mais, dans le même temps, la réflexion demeure plus ou moins concentrée dans le son intérieur, et dans l'acte même qui le détermine, c'est là qu'est l'aperception et l'idée complète qui renferme, pour ainsi dire, en elle-même sa propre matière et sa forme; là est la base et le mobile d'activité de toutes les opérations subséquentes. Quoique la cause d'audition, externe disparaisse, celle d'audition intérieure subsistera et n'aura besoin d'aucune force étrangère pour se mettre en jeu, affecter le sens qui lui est propre, se parler et s'entendre elle-même.

Ici, le souvenir ou le rappel ne doit point commencer par la partie purement audible du son extérieur (qui peut n'avoir fait sur l'organe qu'une impression légère et très subordonnée), mais dans la partie vocale, suivant la détermination volontaire réfléchie qui la fait prédominer dans l'impression directe. Cette initiative de la détermination vocale, effectuée dans l'absence de tout objet ou cause externe, caractérise spécialement le rappel ou la mémoire des sons.

Lorsque l'ouïe est frappée par l'impression reproduite du dedans, l'attention, s'appliquant encore, dans le sens externe, au mode résultant de l'effort, s'identifie avec la réflexion du moi, qui veut l'acte et s'aperçoit en lui. Cet acte vocal, étant constamment suivi ou accompagné d'un résultat sensible qui s'y proportionne, devient plus perceptible dans le produit auditif, que dans le mouvement même qui en est le moyen immédiat et nécessaire. C'est ce produit, indivisiblement uni dans la conscience avec son moyen, qui devient par une habitude première l'objet propre ou apparent du vouloir ${ }^{1}$, et le mobile de la réflexion.

\footnotetext{
Pour les sourds-muets qui auraient appris à articuler ou à prononcer des suites de mots par la méthode première dont usait le célèbre abbé DE L'ÉPÉE (voyez son livre intitulé : Institution des sourds-muets), le mouvement vocal serait le terme propre et exclusif de la volonté ; il n'y aurait point de modification associée résultante, qui pût en quelque sorte détourner le but ou plutôt l'éloigner (car c'est toujours sur le dernier résultat que la volonté paraît se porter, surtout lorsque ce résultat est un mode sensible, et tous les actes ou moyens intermédiaires sont plus ou moins inaperçus, quoiqu'ils aient d'abord été de véritables vouloirs), si de tels sourds parlants venaient ensuite à recouvrer le sens de l'ouïe, ils sentiraient dans le principe, bien mieux que nous ne pouvons le faire actuellement, la distinction réelle qui existe entre le mouvement vocal ou oral, et le son qui en est le produit. Ce serait le premier qu'ils continueraient à vouloir, et le second les étonnerait peut-être d'abord comme une modification imprévue qui se produirait en eux spontanément, jusqu'à ce que la répétition du même acte, suivi constamment du même résultat, eût approprié celui-ci à la volonté. Observons que les mouvements vocaux ou oraux ne peuvent être que simplement réfléchis dans le sourd parlant, comme tout autre produit isolé de la fonction motrice; ils n'ont point, comme en nous, ce caractère de réflexion redoublée; voilà
} 
Il faut appliquer ici ce que nous avons dit précédemment de l'effort inspiratoire, et de l'odeur qui, s'y joignant dans la présence continue d'une même cause externe, devient aussi le but ou le terme propre de la volonté. Mais, dans le cas actuel, les deux éléments sont unis par des liens bien plus intimes : tous deux se rattachant à la même force intérieure, jamais aucune alternative d'impuissance n'altérera leur association, ne séparera la volonté du désir, ni l'attention qui s'attache toujours à la partie la plus sensible de nos modes composés, de la réflexion qui se concentre sur les actes mêmes.

C. Concours de la mémoire et de l'imagination, et comment les deux espèces de réminiscences se réduisent à la même. - La même cause, qui fait que ces deux dernières facultés se confondent ou ne diffèrent guère plus que par le nom dans la perception des sons vocaux reproduits par la volonté, doit faire aussi que les deux espèces de réminiscences, que nous avons distinguées sous les titres de personnelle et de modale, s'identifient également dans le rappel de ces modes actifs. En effet, puisque c'est le son intérieur à reproduire qui est le terme ou le but du vouloir, puisque c'est dans la production même de ce mode que l'activité s'aperçoit et se reconnaît, que l'attention donnée aux résultats ne peut absolument se séparer de la réflexion, inhérente au mouvement ou à l'acte volontaire, la puissance, qui se retrouve nécessairement identique à elle-même dans ce dernier, reconnaîtra, par là même, l'identité de l'autre en le répétant. Le mode rappelé est ici reconnu d'avance, et dans l'instant même où le vouloir est conçu; en un mot la mémoire des sons, comme celle de tous les mouvements répétés avec intention, renferme ou suppose essentiellement la réminiscence du pouvoir, ou de la cause toujours une, comme celle de ses effets immédiats, qui en est bien inséparable : il n'y a, à cet égard, aucune difficulté. Quant aux sons inférieurs que la volonté reproduit, il est bien évident aussi que si les mêmes sons, dont la détermination vocale est déjà contractée, viennent à se renouveler par l'impression directe de la cause extérieure, la réminiscence ne pourra manquer de s'effectuer de la même manière, et en se réfléchissant, pour ainsi dire, du dedans au-dehors, puisque le son entendu est encore rappelé ou répété tout bas.

Mais ici, l'attention, donnée aux modes qui frappent l'ouïe, se sépare encore plus ou moins de la réflexion, qui demeure attachée au son intérieur, et la première pourra avoir sa part dans la réminiscence modale, redevenue distincte alors de la personnelle. Lorsque nous avons écouté, par exemple, attentivement une série de sons formant mélodie, ou une suite de mots articulés (nous ne parlons pas encore des effets de leur association à d'autres idées), suivant que le timbre de l'instrument ou de la voix, qui rend ces sons, saisit et frappe agréablement notre oreille, ou la laisse indifférente, l'attention et la réflexion se

pourquoi ils ne concourent pas mieux et moins bien que les gestes au développement de ses facultés. 
partageront inégalement entre la partie purement audible et la partie vocale, entre ce qui constitue le timbre propre et inimitable des sons, et ce qui en fait le ton, l'accent, la mesure, toutes choses que nous pouvons imiter et reproduire. Lorsque l'oreille n'est plus frappée, et après un certain temps, nous pouvons avoir encore, comme on dit, ce timbre flatteur dans notre oreille et ne point rappeler ou n'avoir qu'un souvenir confus de la partie substantielle dont il était le véhicule ; mais plus souvent, et lorsqu'on a été véritablement attentif, on se souvient bien mieux de la suite des tons, des articulations, que du matériel des sons audibles, ce dernier élément, qui persiste par lui-même dans l'imagination, n'entre dans la mémoire que d'une manière subordonnée et n'est jamais l'objet direct du rappel.

Cependant, nous le reconnaissons très bien dans l'impression directe renouvelée, comme nous ayant déjà frappé, et quoique cette réminiscence qui s'attache à la modification passive, et semble comme elle venir du dehors, ait son premier mobile d'activité dans une attention que la volonté dirige, elle n'en est pas moins distincte de celle qui se fixe plus particulièrement sur les actes répétés, et nous fait reconnaître l'identité de puissance qui est celle même de notre être propre.

Ici donc, comme dans tous les autres cas, la réminiscence personnelle inhérente aux actes peut être séparée de toute réminiscence modale ; mais celle-ci ne peut jamais être sans un degré quelconque de celle-là. C'est ainsi que la sensation ne peut être telle, sans quelque degré d'aperception, quoique celle-ci puisse être séparée, dans l'acte, de toute affection sensible. Tout découle de ce premier principe.

Les analyses précédentes me semblent concourir à montrer clairement, comment les facultés élémentaires de l'intelligence, que l'on pourrait supposer originaires des sensations de l'ouïe en particulier, prendraient leur source réelle dans la partie vocale et vraiment active de ces impressions, et non point dans leur partie audible et passive qui demeurerait toujours inféconde, si elle était isolée. Mais il ne faudrait pas en conclure, que la fonction vocale ait été, dans le principe, et soit toujours également, dans la suite, activée par la même puissance, ni que les mêmes facultés ressortent constamment de son exercice. 


\section{IV. - Fondement d'une distinction entre la voix passive et active. Résultats de ces deux fonctions.}

\section{Retour à la table des matières}

L'instrument vocal est d'abord mis en jeu par la forme aveugle de l'instinct, avant qu'aucune impression ait encore pu frapper l'ouie. Les vagissements de l'enfant, les premiers cris de la douleur, ces sons qui forment la voix naturelle de chaque espèce d'animaux, et qui se retrouvent les mêmes dans ceux qui ont été élevés loin de leurs parents, tous ceux enfin qu'arrachent des affections vives et profondes, hors de toute connaissance et intention, etc., composent un premier langage affectif, très improprement appelé langage, qui n'est tel que pour nous, qui sommes capables de l'entendre, et non point pour l'être sentant, qui ne veut rien exprimer, qui ignore les déterminations de ses organes, comme la plante ignore les rapports et le jeu d'affinité qui dirigent ses feuilles vers la lumière, lorsqu'elle nous annonce, par ses mouvements, le besoin qu'elle a de son influence. Sans doute, tous les êtres de la nature brute organisés et sensibles nous parlent, et (si nous voulons user de métaphore) une langue très animée. Mais où sera l'intention et la puissance animatrice, sinon dans l'intelligence qui reçoit, interprète, vivifie des signes muets par eux-mêmes ? Où sera le jugement et la proposition complète, que l'on va chercher dans la voix native du besoin, ou le cri interjectif de la douleur, sinon dans la tête du profond analyste, qui retrouve partout les produits de sa création?

L'enfant franchit sous nos yeux le passage de ce premier langage inanimé à des signes proprement dits, dont la réflexion naissante commence à fixer la valeur, en même temps que la puissance réelle de l'effort s'en empare et la détermine. Alors aussi les sons vocaux ont changé de nature, et sans doute il faut se reporter à quelque condition ou circonstance organique de leur formation nouvelle, pour apprécier les causes de ce changement, qui coïncide avec plusieurs autres, dans le passage général de l'état purement sensitif à celui d'aperception. (Voyez le chap. II, section I.)

Quoi qu'il en soit, et dès les premiers développements du germe intellectuel, l'enfant commence à s'imiter lui-même dans les sons que sa voix a produits spontanément, avant d'imiter ceux qui peuvent lui venir du dehors. $\mathrm{Ce}$ sont d'abord des sons intérieurs, qui ont distinctement frappé son oreille, et il a dû préluder ainsi par l'aperception ou la réflexion commencée des actes dont il 
est et se sent cause, à l'aperception des modes auditifs, dans lesquels il est et doit se sentir bientôt comme effet de quelque autre cause qui n'est pas lui. Ici l'ordre des deux termes, sur lesquels se fonde le rapport primitif de causalité, est direct et naturel. Il n'est point interverti comme il paraît l'être dans d'autres sens, dont on a, trop exclusivement peut-être, consulté le témoignage.

Si la fonction vocale suivait toujours sa première détermination, si ses produits aveugles conservaient leur caractère affectif originel, l'exercice de l'ouïe, avec la voix ou sans elle, serait de nul emploi dans la formation ou le développement des facultés intellectuelles. Il en serait de même si l'ouïe, paralysée dès la naissance, ne pouvait recueillir et juger les premiers produits des déterminations vocales, de quelque manière que celles-ci s'effectuassent dans la suite, et il ne me paraît point douteux qu'on ne doive attribuer à cette dernière circonstance l'incapacité de réflexion qui caractérise les sourds-muets, privés, dès l'origine, du sens qui est en nous le premier mobile de toute réflexion.

De là il suit que nous devons distinguer encore dans les fonctions mêmes de la voix, outre la partie active dont nous avons déjà reconnu les produits, une autre partie qui, originellement passive, peut continuer à l'être ou le redevenir plus ou moins, après avoir été activée en partie par la volonté. Ces deux fonctions se distinguent très précisément en ce que l'une imite toujours des sons reçus du dehors, ou s'imite sciemment elle-même, tandis que l'autre n'imite rien, et n'est, comme tout mouvement instinctif, que le signe involontaire et inaperçu de quelque affection qui la détermine d'une manière immédiate.

Parmi les sons imitables et volontairement imités, il en est qui se rapprochent et ne diffèrent presque pas de la voix native, et du langage purement affectif. Ceux-là composent encore la langue des sensations ; naturellement appropriés à la fonction vocale passive, à peine ont-ils besoin d'être appris, à peine leur production ou leur rappel commandent-ils quelque léger déploiement de l'effort. Il en est au contraire, qui, s'exécutant sur les touches les plus rudes ou les plus résistantes de la voix ou de la parole, exigent une assez longue éducation et le concours de toute l'activité volontaire ${ }^{1}$.

\footnotetext{
Il me paraît qu'on doit distinguer une fonction purement vocale, et une fonction orale, qui concourent ensemble dans l'exercice de la parole articulée, mais qui n'en ont pas moins des caractères très distincts, relativement surtout à la part plus ou moins active qu'y prend la volonté. La production de ce que nous appelons voyelle ou voix, tient uniquement à un certain mode d'émission de l'air hors des poumons; c'est une manière de respirer ou d'aspirer plus marquée qu'à l'ordinaire. Dans les soupirs, les sanglots, le bâillement, le rire, etc., comme dans ces interjections que les fortes passions arrachent, ce sont des voix ou des sons justement dits inarticulés, qui se forment sans la participation, souvent même contre l'action expresse de la volonté. Cette puissance influe plus directement sur le resserrement ou la dilatation active de la glotte, qui modifie l'air à sa sortie des poumons et détermine ainsi le ton ou les différents degrés du grave à l'aigu des sons vocaux. Quant au timbre de
} 
On conçoit aisément que les premiers favoriseront, par leur nature propre (et indépendamment de tout effet ultérieur d'association), le développement de l'imagination et des passions, tandis que les autres, concentrant la pensée sur elle-même, s'allieront à tous les progrès de la réflexion et des facultés qui en dépendent; mais il suffit d'indiquer les fondements d'une distinction, dont les conséquences dérivées nous entraîneraient bien loin de notre but présent ${ }^{1}$.

Si après avoir cherché les rapports d'origine des facultés intellectuelles avec l'exercice particulier de l'ouïe et de la voix, nous voulions déterminer la nature ou le caractère spécial des idées qui se réfèrent à la même source, nous trouverions qu'indépendamment de la connaissance extérieure, qui ne se rallie que très indirectement à l'exercice de ce sens, il peut fournir un champ vaste et plus approprié aux idées réfléchies.

\section{V. - Caractère des idées qui dérivent de l'exercice de l'ouïe et de la voix.}

\section{Retour à la table des matières}

Il n'en est point du sens de l'ouïe comme de celui de l'odorat : dans ce dernier, les modifications varient incessamment sous l'impulsion continue de la même cause et le déploiement d'une force identique ; elles sont fugitives et très

ces sons, il tient à des dispositions organiques, sur lesquelles la volonté ne peut rien, c'est ce qui fait qu'un homme ne saurait guère jamais déguiser entièrement le caractère de sa voix, quoiqu'il l'élève pu l'abaisse à son gré.

Mais l'articulation proprement dite dépend entièrement de la fonction orale, et, dans cette partie du phénomène de la parole, toutes les parties mobiles de la bouche, la langue, les dents, les lèvres jouent un rôle éminemment actif. La volonté a dû s'appliquer dans l'origine, et par une détermination expresse, à mouvoir en détail chacune de ses parties, de la manière propre à former tel son articulé. Aussi, ces sons, quoique devenus profondément habituels, conservent-ils toujours leur principal caractère originel, pendant que les voix, passivement émises, vont exciter la sensibilité de l'ouie, ou mettre sympathiquement en jeu d'autres organes dans celui qui les entend. Les articulations doivent être écoulées volontairement, pour être imitées de la même manière, et ce n'est qu'en vertu de cette imitation qu'elles sont distinctement perçues. Remarquons aussi que dans l'action régulière de la pensée, qui se fonde sur une sorte de discours que nous nous adressons tout bas à nous-mêmes, la fonction vocale est sans exercice ; mais les touches orales sont frappées et mises en jeu. C'est par ce moyen que l'organe intellectuel s'électrise, que l'attention se fixe, et que la pensée trouve, pour ainsi dire, un point d'appui pour réagir sur elle-même.

On peut déduire de ces considérations qui paraissent d'abord si minutieuses, des résultats très importants sur l'origine des langues, leur caractère et leur génie, les habitudes que leur usage fait naître et éternise parmi les nations qui conservent leurs langues indigènes; mais ces objets doivent être traités séparément. 
peu persistantes, quand la cause a disparu et quoique l'action continue. Mais l'organe auditif peut conserver d'abord, en vertu de certaines dispositions vibratoires qui lui sont propres, les ébranlements sonores reçus du dehors, et la voix y réunissant, pour ainsi dire, ses produits d'accompagnement, donne aux mêmes impressions une persistance qui n'a presque d'autres bornes que celle de la volonté.

Les sons perçus dans leur succession correspondent chacun à un mouvement particulier, qui, après en avoir opéré la distinction complète dans le sens, en prépare le rappel exact, suivant le même ordre. Il ne peut y avoir de telle liaison fixement établie dans la mémoire, sans qu'il y en ait une parallèle et concomitante dans le jugement qui s'opère par la comparaison ou l'association qu'ont entre eux les sorts successifs. L'attention, qui suit les impressions directes, sera le mobile de la première association ; la réflexion, qui s'attache aux modes actifs intérieurs, sera la cause efficiente de la seconde.

Pendant qu'une certaine suite de sons frappe directement l'ouïe accoutumée à les entendre, une série parallèle se déroule intérieurement dans la mémoire. La coïncidence, l'analogie ou le contraste de ces termes ressortent ici de l'exercice simultané de l'attention et de la réflexion, indivisibles dans leur source. Un même son peut persister dans l'oreille comme une sorte de base continue, pendant que la voix parcourt tous les sons dérivés et réciproquement. Il peut donc y avoir ici deux ou plusieurs modes perçus, d'abord successivement, ou les uns hors des autres, qui le seront ensuite simultanément dans le même ensemble et sans se confondre : ainsi, dans le double exercice de l'ouïe et de la voix, la mélodie prépare l'harmonie, qui lui sert de base à son tour. Observons pourtant que les déterminations ou les actes de la volonté, rie pouvant être que successifs, on pourrait douter, si la simultanéité apparente des sons qui coïncident harmoniquement, n'est pas plutôt une succession très rapide, mais la disposition vibratoire du sens auditif externe ou de l'organe intérieur de l'imagination, pouvant toujours réellement coïncider avec l'acte vocal volontaire, la difficulté est levée par cette circonstance même ${ }^{1}$.

Quoi qu'il en soit, puisqu'il y a des sons comparés, il y en a de perçus simultanément, et puisqu'il y a de telles perceptions d'ensemble, il y a donc aussi des idées composées et abstraites, de véritables compositions et abstractions modales, résultats de l'exercice d'une activité réelle; je dis résultats ou produits, parce qu'on ne saurait distinguer, comme ont fait Locke et plusieurs autres métaphysiciens, des facultés particulières de composer et d'abstraire ; c'est par la vibratilité du sens externe, ou de celui de l'imagination passive (qui entretient les ébranlements successifs), et en même temps par

C'est là, ce me semble, le fondement de la solution d'une difficulté élevée depuis longtemps parmi les métaphysiciens, sur la question de savoir, s'il peut réellement y avoir plusieurs idées présentes à la fois. Nous reviendrons ailleurs sur cet objet, mais il importe de ne pas perdre de vue le principe. 
l'attention active qui saisit les coïncidences, que s'effectuent surtout les compositions des modes; c'est la réflexion qui les sépare, les abstrait d'un même ensemble, et qui, s'attachant ici en particulier aux modes intérieurs, produits exclusifs de l'activité individuelle, parfait la ligne de démarcation entre les abstractions réfléchies et les abstractions modales. Ces deux sortes d'abstraction qui sont distinctes en effet, comme le sentiment propre de l'acte l'est de celui d'un résultat sensible mixte, s'identifient ici dans l'acte exclusif du rappel, ainsi que nous l'avons observé des deux sortes de réminiscences.

Dans le rappel volontaire des sons oraux, le mouvement ou l'acte vocal ne peut être séparé de son produit auditif : dans l'un et l'autre à la fois se trouvent compris le sentiment de puissance ou de causalité (exemplaire premier de toute idée de cause, ou force productive étrangère), celui d'unité et d'identité dans le temps, ou la durée distinctement mesurée par des modes successifs, aperçus les uns hors des autres, par conséquent d'idée de pluralité dans ces modes, et celle de totalité dans un intervalle de temps qu'ils occupent; les idées de réalité ou de privation, celles d'existence et de non-existence, dérivent immédiatement du contraste perçu entre les sons qui continuent ou cessent par l'action de la volonté, et ceux qui surviennent ou persistent quand elle n'agit plus, etc. Tous ces modes potentiels, que Kant distingue sous le nom de catégories, sont en effet inhérents au déploiement de l'action volontaire sur des termes appropriés, mais ils ne sauraient être conçus hors de ce déploiement, ni avant lui, par conséquent hors des conditions originelles qui le déterminent, des instruments qui l'appliquent.

Les catégories sont de véritables abstractions; les modes potentiels dont nous parlons peuvent être dits aussi abstraits, en tant qu'ils sont séparés par la réflexion des impressions sensibles qui coïncident avec eux, et qui sont ainsi perçues sous divers rapports de succession ou de simultanéité, d'analogie, de ressemblance, d'identité, etc., rapports que nous transportons aux choses ou aux modifications passives, mais qui ont leur source réelle dans des actes qui viennent uniquement de nous-mêmes. Il y a là matière d'une véritable décomposition, dont les produits isolés peuvent être dits aussi abstraits. Mais en tant que l'on n'a égard qu'aux actes volontaires produits ou rappelés, et aux modes qui en résultent immédiatement, sans le concours actuel d'aucune force étrangère, ces modes intimes, sous lesquels la même existence personnelle est continûment ou successivement aperçue, excluent toute composition hétérogène, et ne peuvent être dits abstraits en aucune manière. Ces idées simples de la réflexion diffèrent donc réellement des abstractions réfléchies, et encore plus des idées de modes sensibles que l'attention objective sépare d'abord et groupe ensuite de nouveau, pour en former les types de genres, d'espèces, etc. Les signes de celle-ci ont toujours, dans notre langage, une généralité plus ou moins étendue ; les signes des premières conservent toujours l'individualité précise, qui est dans l'essence même de la chose signifiée. Rien de plus dangereux en philosophie que de confondre tout cela sous le nom 
d'abstractions. Rien de plus important que de rattacher les différences à des conditions intelligibles prises dans la nature.

Nul autre sens que celui de l'ouïe n'était plus propre à fournir des moyens de distinction des trois espèces d'idées dites abstraites, que nous venons de noter. L'exercice de ce sens, uni à la voix, est surtout bien remarquable en ce qu'il est le seul, qui fournisse à l'individu les moyens directs de se modifier luimême sensiblement, par un seul acte de sa volonté, et sans aucun autre concours : seul, entre tous, il concentre sur ses actes ou modifications propres, le sujet sensible et moteur, au lieu de l'entraîner au dehors. Dans les autres sens, le mouvement ou l'effort ne part de l'individu que pour se fixer sur un terme extérieur, et s'y absorber en quelque sorte. Ici, il ne part du sujet pour s'appliquer au terme organique, que pour se réfléchir encore dans le premier, sous une forme nouvelle et plus sensible. Ailleurs, la volonté fixant un but étranger, et traversant en quelque sorte son moyen propre, pourra se perdre de vue, et finir par s'ignorer elle-même. Ici le but même rend le moyen plus présent, et l'agent se retrouve, s'aperçoit, se reconnaît également dans tous deux

Bientôt peut-être, ces modes que nous venons de considérer comme mobiles propres d'activité, et termes exclusifs du vouloir, ne paraîtront plus que comme des moyens accessoires et éloignés ; mais leur caractère intrinsèque restera. En s'associant avec nos modifications et nos idées de tout genre, la parole articulée ne leur communiquera un caractère plus réfléchi, que parce qu'elle le porte en elle-même. Elle ne donnera sur toutes une sorte de prise à la volonté, qu'en tant qu'elle est un mobile ou un instrument immédiat de cette puissance, qui s'y trouve originellement renfermée ; elle ne sensibilisera enfin les modes les plus intimes de l'intelligence en les activant, que parce qu'elle est, elle-même un acte intellectuel sensibilisé. C'est pour avoir négligé l'analyse de ces propriétés, inhérentes aux perceptions auditives et vocales, considérées en elles-mêmes et hors de toute association, que les métaphysiciens ont si souvent méconnu les fonctions plus importantes, qu'elles remplissent en qualité de signes. De là aussi, tant de nuages répandus sur la source même de nos facultés. Je justifierai encore ainsi, au besoin, les détails peut-être trop minutieux, en apparence, dans lesquels je viens d'entrer. 


\title{
Chapitre IV
}

\author{
Analyse de la vision. - Des facultés originelles \\ et des idées qui s'y rapportent.
}

$\underline{\text { Retour à la table des matières }}$

Les rayons lumineux, qui impressionnent directement la rétine, occasionnent une affection simple qui ne saurait d'abord se rapporter ni à un objet, comme cause, ni à un sujet un qui puisse être censé la sentir ou la percevoir, à moins qu'on ne supposât d'une part la personnalité (ou comme le disait Leibnitz, l'unicité), constituée soit par sa propre nature, soit par quelques conditions particulières qui ne sont pas sûrement dans l'hypothèse abstraite d'un œil vivant ou animé, et qu'on ne supposât aussi d'autre part quelque idée innée, ou quelque connaissance anticipée de cause ou d'objet externe qu'il est difficile d'admettre comme idée, la première fois que la lumière agit sur l'œil, puisqu'il faudrait la faire remonter avant la connaissance même.

Il me paraît évident, et d'après même tout ce qu'a dit un grand maître sur ce sujet, que les premières sensations de lumière ou de couleur, n'ont point en elles-mêmes, ou dans leur propre nature, un caractère de relation, qui les rende propres à informer le moi (ou la personne préconstituée par l'hypothèse) de quelque existence étrangère, ni même, dans mon sens, à lui faire apercevoir ou connaître la sienne propre.

On tranche le nœud de toute difficulté, en disant avec les Kantistes, que la première couleur perçue est dans l'espace. Cela peut être vrai de la perception, mais il faudrait avoir prouvé d'abord, qu'il y a une perception plus ou moins distincte, correspondante aux premières impressions des rayons lumineux, ce qui n'est assurément rien moins que probable. 
L'action, que la lumière exerce d'abord, pour ainsi dire en masse, sur l'organe, ne peut occasionner qu'une sensation générale plus ou moins affective, de même nature que celle que nous éprouvons, en passant de l'obscurité au grand jour. L'œil est fortement impressionné et nous ne voyons rien. L'enfant, qui vient au monde est affecté de même par la lumière et ne voit point encore : assez longtemps après, ses yeux demeurent fixes, ternes, incertains, ils voient déjà mais ne regardent point. La flamme perceptive, qui leur donnera dans la suite une expression si remarquable, n'y a pas encore brillé.

\section{I. - Double exercice de la vue. Conditions de l'activité et de la passivité originelle de ce sens.}

\section{$\underline{\text { Retour à la table des matières }}$}

Admettons l'existence des conditions de motilité volontaire, sur lesquelles nous avons cru pouvoir fonder le passage d'un état purement affectif à celui d'aperception personnelle. Supposons ce passage franchi, et faisant abstraction des secours que la vue retire des autres sens, qui concourent directement avec elle dans l'état naturel, tâchons encore de signaler le caractère des facultés originelles, qui pourraient se rapporter à l'exercice particulier de ce sens.

Dans l'effort général, qui constitue l'état de veille, se trouve comprise, et comme enveloppée, l'action particulière qui relève ou baisse à volonté les muscles des paupières, ouvre ou ferme l'œil. L'individu peut bien d'abord ne pas s'apercevoir guère plus de la liaison de cet acte non intentionné avec les impressions de lumière, qu'il n'aperçoit la liaison des odeurs avec le mouvement naturel de respiration. Cependant, dès qu'il sort des opérations de l'instinct, et qu'il commence à y avoir pour lui quelque expérience possible, la première liaison ne pourra demeurer complètement ignorée.

A. Comment l'individu peut être porté à s'attribuer les modes de couleur. Expérience qui doit promptement le désabuser. - L'impression trop vive de la lumière a contraint les yeux de se fermer. Ils restent quelque temps dans cet état, ils s'ouvrent de nouveau et l'individu est modifié. Il baisse les paupières, et les relève encore par un acte exprès de sa volonté, et il sent qu'il crée ou anéantit par lui-même ses modifications, il est bien près de les attribuer à sa propre puissance et de les vouloir, comme il veut l'action même qui les précède ou les accompagne. 
Cependant le sentiment de puissance ou de causalité personnelle ne peut être supposé ici comme étant aussi intime, aussi vrai même, que dans l'odoration active, telle que nous l'avons considérée et il n'y a aucune comparaison à faire avec ce qui a lieu dans la production des sons vocaux.

Une impression quelconque de lumière suit bien constamment l'acte de la volonté qui tient les yeux ouverts, et les ténèbres arrivent dès qu'elle les ferme. Mais il n'y a point une proportion assez marquée entre l'acte et son produit. Le premier n'est que d'un instant et l'autre se continue ou persiste par lui-même ; celui-ci s'accomplit d'une manière uniforme, et n'est point susceptible même de différents degrés (comme l'était, par exemple, l'effort inspiratoire). Celui-là peut varier, se transformer de différentes manières, s'aviver ou s'obscurcir. Ce n'est point ainsi que se fonde la relation intime et constante de la cause à l'effet.

Parmi les impressions, qui se succèdent ou se combinent diversement sous la même action visuelle, il en est qui flattent ou réjouissent l'organe, d'autres qui le blessent ou le repoussent. L'individu voudrait retenir les unes, et elles lui échappent; il tend à fuir les autres, et souvent elles préviennent son action. Le voilà donc conduit, soit par le contraste d'un état actif et d'un état passif, comme dans l'exercice de l'ouïe, soit par la résistance même au désir (qui naît de la limite sentie du pouvoir), comme dans l'exercice de l'odorat, le voilà, disje, conduit à la notion confuse d'une cause ou force non moi qu'il conçoit ou croit encore sur le modèle de sa puissance, et on ne peut lui accorder la moindre expérience, la moindre faculté, au-dessus de la simple affection, sans que cette idée de cause étrangère ne dérive ici du premier exercice des facultés visuelles (Condillac me paraît avoir trop négligé cette considération).

B. En quoi il doit continuer à se reconnaître actif. - Cependant, il est des circonstances qui pourraient seconder et justifier plus ou moins, dans un exercice actif de la vue, le penchant qu'a l'être moteur et sensible, pour s'approprier des modifications auxquelles sa puissance concourt. En effet, l'action visuelle est loin d'être bornée aux mouvements volontaires, qui élèvent ou abaissent plus ou moins les paupières, et modifient ainsi, jusqu'à un certain point, l'impression des rayons lumineux, il y a plusieurs autres mouvements qui servent à fixer, à diriger l'organe, à raccourcir, à allonger son diamètre, pour faire converger les rayons au point convenable et opérer une vision distincte. Ces actes, que la volonté commande et s'approprie, sont aperçus ; eux seuls constituent cette partie de la fonction visuelle qui est susceptible d'une véritable éducation. Avant de parvenir à ce degré de facilité, qui maintenant les rend comme insensibles, ils ont dû s'accomplir dans le principe avec un effort, dont le sentiment particulier, associé avec l'affection lumineuse simple, a pu constituer une véritable sensation composée. De là une vision active dont les produits sont aperçus, distincte de la vision passive, où les impressions sont directement et immédiatement senties. L'individu, en le supposant même borné 
à des sensations de cette espèce, ne pourrait être dit dès lors les devenir, il serait une personne, sujet un de l'effort moteur, dont le terme est senti dans son inertie propre, et les impressions particulières dont il est le siège. C'est à ce terme que serait rapporté ce qui varie ou ce qui est produit hors de la puissance, toujours identique à elle-même. L'individu ne se sentira donc pas varié $^{1}$, lorsqu'un mélange de diverses couleurs est présent à l'organe, il sentira tout au plus cet organe varié, lorsqu'il n'a pas encore appris à rapporter ses modifications à un terme étranger : mais comment pourrait-il être tout à la fois le tableau coloré et le spectateur ou le juge ? Que l'impression visuelle quelconque, confuse ou distincte, uniforme ou variée, soit en lui, c'est-à-dire dans l'organe, ou hors de lui dans l'espace, toujours est-il vrai que dès qu'il l'aperçoit, elle n'est pas lui, son moi n'est pas identifié avec elle.

C. Signes auxquels on reconnaît une vision active et passive.- Il n'y a nulle autre part de distinction plus marquée que celle qui existe entre les modes correspondants à la vision active, et ceux qui correspondent à la vision passive, soit que nous les considérions intérieurement ou par rapport à nous-mêmes, soit que nous ayons égard surtout à l'effet de représentation objective.

Cette distinction entre le voir simple et le regarder, qui est expressément notée dans le langage, se fonde entièrement sur l'absence relative ou la présence immédiate de la volonté. Or, il n'y a de volonté immédiatement présente, que là où se trouvent les conditions, les instruments ou les termes de motilité.

Lorsque la vision est activée par un vouloir exprès, les yeux prennent une expression particulière qui manifeste la vie, et comme le feu pénétrant de l'intelligence (ignis intelligens) ${ }^{2}$. Ce feu n'est point excité du dehors, ce n'est

1 Condillac voulant exprimer l'état de la statue lorsqu'elle est affectée à la fois par diverses couleurs qu'elle ne rapporte encore qu'à elle-même (il fait abstraction du rapport au siège organique), dit que la statue se sent variée, expression très ingénieuse, mais qui suppose la nullité d'aperception, hors de laquelle il ne saurait y avoir de véritables facultés.

2 Il faut bien prendre garde à ne pas confondre cet effet que produit sur la vue, comme sur tous les autres sens perceptifs, l'influence actuelle et positive de la volonté, avec d'autres effets produits par les passions et les appétits, sur les mêmes sens quelquefois mais plus souvent sur les organes sensitifs en particulier. Il y a quelques apparences communes dans ce phénomène, mais assez de différences, pour qu'on ne doive pas les identifier absolument dans leur principe. Pourrait-on comparer par exemple cette excitation particulière que produit, dans le sens du goût, l'appétit de certains aliments dans telles dispositions de l'estomac, la susceptibilité que prend l'odorat lorsque le sens de l'amour, devenu un centre puissant de réaction, lui communique son influence, avec les produits de l'irradiation volontaire dont nous parlons, et n'y a-t-il pas une différence bien remarquable entre l'expression intellectuelle que prend le regard dans l'attention active, et celle que les diverses passions donnent à la physionomie, et surtout aux yeux, qui s'enflamment ou languissent, s'animent ou s'obscurcissent tour à tour ? etc.

$\mathrm{Si}$, dans ces derniers cas, les forces sensitives prédominent, pour parler comme les physiologistes, l'initiative d'action n'appartient-elle pas à la force motrice dans l'autre ? 
pas non plus dans l'organe sensitif qu'il s'allume spontanément, quoique ce soit sur lui que se porte son influence. L'action qui va animer le regard, et le pointer vers son objet, n'est point irradiée immédiatement du centre sur les parties nerveuses (comme par un effet de réaction, qui peut avoir lieu dans d'autres cas très différents), mais d'abord sur les instruments propres de motilité qui agitent le globe oculaire, le pressent, etc., l'électrisent véritablement par communication et lui impriment ces modifications si favorables à la perceptibilité distincte.

Si l'on écarte l'effet de ces instruments musculaires (que la nature n'eût pas tant multipliés dans l'organisme visuel, si elle ne l'avait destiné qu'aux fonctions pures de la sensibilité), on n'aura plus qu'une sorte de vision passive, ou plutôt des sensations intérieures toujours confuses, mais plus ou moins vives suivant le degré précis des impressions de lumière ${ }^{1}$.

D. Distinction des deux fonctions et de leurs produits, indépendante de toute autre sensation. - Quand même le sens de la vue s'exercerait isolément, et hors du concours de tout autre sens auxiliaire, les deux fonctions précédentes n'en seraient pas moins distinctes entre elles, et dans leurs produits, et s'il n'y avait que des sensations confuses et affectives correspondantes à l'une, pourquoi n'y aurait-il pas dans l'exercice de l'autre, de véritables perceptions des modes colorants, aperçus les uns hors des autres, soit dans l'organe même (que la volonté ne peut diriger sans apprendre à le connaître comme terme d'action et siège impressionnable), soit flottante, au devant de l'œil, dans le vague de l'espace, comme ces images, ces suffusions scintillantes, que nous voyons ainsi souvent hors de l'œil, dans certaines dispositions nerveuses ? Pourquoi les ébranlements et l'électrisation réelle, que la volonté communique immédiatement aux fibres de la rétine, n'auraient-ils pas dans tous les cas des effets différents de ceux qui sont médiatement produits dans ces fibres par les impressions, quand on fait abstraction de l'influence motrice ? Pourquoi n'y aurait-il pas une vertu directement représentative, attachée à cette dernière influence comme il y en a une aperceptive ? Enfin, dès que l'on suppose un être capable d'exercer quelques opérations intellectuelles, de se faire différentes espèces d'idées, par l'exercice de la vue seule, pourquoi tant limiter en lui, d'un

J'ai éprouvé souvent la différence qu'il y a entre les impressions que font sur la vue les mêmes objets éclairés, suivant que l'organe s'offre, pour ainsi dire, passivement à ces impressions, ou qu'activé par la volonté, il les prévient et va au-devant d'elles. En laissant, par exemple, tomber la vue avec distraction sur une fenêtre exposée au grand jour, puis la portant sur d'autres objets, j'ai encore quelques instants présente l'image distincte de la fenêtre qui flotte devant mes yeux : si je regarde fixement ce même objet, il n'y a rien de pareil, et les ébranlements ne persistent plus d'une manière sensible ; ce qui prouve, ce me semble, qu'il y a une sorte d'élasticité propre à l'organe visuel, qui se lie à l'imagination passive, hors de toute influence de la volonté, mais que cette imagination d'une part n'est que la sensation même continuée, et ne suppose aucune faculté nouvelle; tandis que l'influence volontaire d'autre part change les phénomènes de la vision simple, et y fonde des facultés d'un autre ordre. 
autre côté, les résultats de l'expérience qu'il pourrait acquérir ? Et qui sait toutes les différentes manières de voir et de regarder, dont ce sens, réduit ainsi à sa propre expérience, pourrait contracter l'habitude ?

E. La propriété représentative inhérente à la vue ne diffère point des affections de la sensibilité, comme des modifications. - Quoi qu'il en soit, dès que nous admettons une perception de couleurs (très différente de la sensation produite par le choc des rayons en masse), que ces couleurs soient rapportées à l'organe, comme terme immédiat auquel la volonté s'applique, ou sur cet organe comme par effet de pression, ainsi que le disait l'aveugle de Cheselden après avoir été opéré, ou enfin dans un espace, peu nous importe pour le moment, il est toujours vrai que le mode perçu d'une manière quelconque n'a point du tout le caractère d'une affection simple avec qui l'être sentant soit identifié, ni celui d'un acte que l'individu puisse complètement s'attribuer comme sujet de l'effort.

Il n'en est point ici comme dans l'action inspiratoire et vocale et leurs produits respectifs. Les odeurs et les sons directs ne pouvant être dépouillés de tout caractère affectif, ces modes ne se circonscrivent point nettement dans un siège particulier; l'impression en est plus ou moins générale, et c'est celle-ci même, qui peut être dite sentie. Dans la vision, au contraire, l'impression de la lumière doit être comme nulle pour qu'il y ait quelque perception de couleur, soit au dedans, soit au dehors de l'organe ; rien n'est senti, mais quelque chose est représenté dans cet organe ou par lui, et ce quelque chose, objet ou image, n'est point le moi, ni ne peut s'approprier à lui comme puissance motrice, pas plus que comme vertu sentante.

Telle est cette fonction représentative, propre au sens de la vue : nous ne pouvons en assigner les conditions premières qui échappent à toute observation, puisque nous ne voyons point ce qui nous fait voir. Tout ce qu'il nous est permis de concevoir ou supposer ici, c'est que la force extérieure de la lumière, se rapprochant par sa nature de celle qui irradie d'un centre unique le mouvement et l'action aux organes, concourt avec elle, et la rencontre directement sans exciter la sensibilité nerveuse ; que la vision, dépendant toujours du concours des deux forces, est passive ou active, suivant le degré de prédominance de la cause extérieure, mais que l'initiative appartenant essentiellement à cette dernière, et l'autre ne pouvant qu'équilibrer, pour ainsi dire, son impression dans le complément de l'activité visuelle, il doit entrer toujours beaucoup de passivité de notre part dans tout effet de représentation.

Nous verrons bientôt que le toucher change, en quelque sorte, ces proportions, mais l'accord parfait de ces deux sens, les secours mutuels qu'ils se prêtent, et l'analogie des facultés qui se rattachent à leur commun exercice, 
tiennent à la similitude originaire de leurs procédés, à la manière dont ils s'appliquent à leur objet, ou dont leur objet s'y applique ${ }^{1}$.

Dans la vision même la plus active, le déploiement de l'effort sur un terme peu inerte, ou doué par lui-même d'une mobilité particulière, et très rapproché d'ailleurs du centre de mouvement, n'est presque point perceptible dans son produit immédiat, mais uniquement dans ce résultat secondaire, le mode représenté auquel la volonté ne concourt que d'une manière subordonnée ; c'est à ce mode, comme à son élément propre, que l'attention s'attache ; c'est en lui qu'elle existe, il n'y a point de réflexion proprement dite, à moins qu'on n'appelât ainsi cette sorte de réflexion spéculaire qui rejaillit, pour ainsi dire, d'un objet sur un autre, sans jamais se concentrer; qui, mettant le sujet en relief hors de lui-même, fait qu'il croit se voir où il n'est pas, et ne sait plus s'apercevoir ni se retrouver où il est, ignore ainsi sa place, sa mesure réelle, et poursuit son image dans le fantôme mobile qui lui échappe.

La manière dont l'attention s'applique au mode représenté, et l'absence de tout effet de réflexion dans ce mode, déterminent par une suite nécessaire le caractère des facultés et des idées qui se rattacheront particulièrement à l'exercice de la vue.

\section{II. - Caractère des facultés représentatives.}

$\underline{\text { Retour à la table des matières }}$

Dans l'aperception visuelle, la volonté est bien en partie une force électrisante, mais la matière électrique lumineuse ou colorée vient d'ailleurs, ou se produit quelquefois spontanément dans l'organisation, par une vertu bien indépendante de la volonté. Celle-ci ne crée point la lumière ou n'imite pas les couleurs, comme la voix crée et imite les sons qui ont extérieurement frappé l'oreille, et le regard ne fait pas la vision dans les ténèbres, comme l'activité vocale fait l'audition dans le silence.

A. Imagination passive proprement dite applicable au sens de la vue. L'objet coloré venant de s'éloigner, son image peut encore rester présente par la vibralité éminemment propre à cet organe ; la même image peut se reproduire encore, après un intervalle quelconque; en vertu d'une telle disposition vibratoire, mise en jeu spontanément, l'effet de représentation est toujours égal à lui-même, la copie se confond avec l'original, l'illusion avec la réalité ; mais

C'est ce que LUCRÈCE exprime très précisément dans ce vers : necesse est consimiti causâ visum tactum que moveri. 
où est ici le type original et réel ? Sans doute, ils se sont donné un grand avantage, les idéalistes qui ont pris ce sens pour terme de certaines observations particulières, adroitement généralisées; ils ne pouvaient pas mieux choisir.

Il n'y a point de réminiscence attachée à cette vision passive externe ou interne : peu importe que la cause soit présente ou éloignée, nouvelle ou ancienne, la vivacité de l'image peut s'accroître ou éprouver quelque déchet, mais la fonction qui représente directement dans ce cas, n'en est pas moins dénuée de tout acte intellectuel, et ne peut être dite souvenir, à moins qu'on ne change le sens des termes. Si le sentiment propre de l'être revit ou se conserve dans de tels produits imaginaires, c'est qu'il y a un autre fondement à l'aperception, comme à la réminiscence de cet être.

B. Imagination jointe à la réminiscence qui prend le caractère exclusif de modale. - Lorsque l'impression extérieure visuelle a été activée en premier lieu par l'attention, le même mode étant reproduit par la cause extérieure (supposée connue ou inconnue), la personne identique pourra revivre particulièrement dans la répétition du même acte, sur laquelle elle se fonde en premier lieu. Mais comme l'aperception se trouvait alors tout entière dans le mode, résultat partiel de l'effort, et non point dans l'acte même, ce sera aussi dans le mode reproduit, plutôt que dans l'acte qui peut concourir de nouveau à la représentation, que l'identité sera reconnue. La réminiscence, qui, dans le cas présent, se joint à l'image, en proportion de l'activité déployée dans sa formation première, est donc revêtue du caractère particulier qui la constitue modale (nous pouvons dire dès à présent objective). Or, comme cette réminiscence modale, attachée au rappel ou à la répétition active des mêmes sons, se confondait ci-devant avec celle que nous avons appelée personnelle, en prenant son caractère réfléchi, ici ces deux réminiscences se confondent encore dans la reproduction des mêmes images, mais c'est la personnelle qui est absorbée, pour ainsi dire, dans la modale, et s'objective avec elle. Ce n'est point notre être que nous reconnaissons en effet dans la perception d'un objet qui se montre à nos regards, après y avoir été soustrait plus ou moins longtemps ; c'est cet objet que nous reconnaissons, c'est lui qui paraît avoir duré, c'est sur lui et souvent, sans le moindre retour sur nous-mêmes, que se cumulent tous les instants successifs, et les changements nombreux qui séparent deux points éloignés, pris dans notre propre durée.

Dans l'absence des objets, et hors des dispositions organiques qui reproduisent spontanément les images, hors aussi des moyens auxiliaires qui approprient, jusqu'à un certain point, cette reproduction à la volonté, il n'y a point de rappel direct, point de mémoire proprement dite des modes de couleurs. La vue est le sens particulier de l'imagination, et c'est précisément parce qu'il a usurpé une sorte de domination sur toutes les facultés, par la continuité et la facilité de son exercice, que nous avons d'autant moins de dispositions à réfléchir, apercevoir et reconnaître ce qui est en nous, ou qui est 
nous, que nous en avons davantage pour représenter, imaginer et reconnaître ce qui est au dehors.

La réminiscence objective rattache toujours à quelque $l i e u$, à quelque point de l'espace, nos modifications les plus intimes, et jusqu'aux produits de notre création : c'est là qu'une pente invincible nous entraîne toujours à aller les étudier, les signaler de nouveau. Ce sont les objets étrangers que nous interrogeons sur le secret de notre pensée, c'est portés sur les ailes de l'imagination qui tantôt nous élève jusqu'aux cieux, tantôt nous fait descendre dans les abîmes de la terre, que nous croyons la saisir et la contempler. Faut-il s'étonner que la science de nos facultés ait paru se confondre avec celle de nos idées, dont le sens de la vue fournit en effet la partie la plus considérable et la plus influente ? Faut-il s'étonner qu'en prenant ce sens pour modèle, et généralisant les résultats déduits de ses fonctions particulières, toute l'intelligence humaine ait été ramenée par les uns à la sensation ou représentation passive, qui nous constitue en dépendance essentielle des objets, et réduite par les autres à un système d'idéalisme qui anéantit ces objets euxmêmes?

La vue est éminemment le sens qui compose ; c'est par là même qu'il est celui de l'imagination ${ }^{1}$. Sa vibralité ou son élasticité propre tend toujours à faire rentrer les uns dans les autres les modes mêmes qui se succèdent, et à les représenter simultanément dans le même ensemble ; c'est par un tel effet que le passé redevient présent, ou se confond avec lui dans la même perspective, l'imagination nous cachant la distance par les vives couleurs dont elle peint des objets éloignés ; c'est ainsi qu'elle impose à ses créations dans l'espace et le temps un ordre particulier, où tout se règle sur la vivacité des impressions, et non plus sur la réalité des termes qui sont véritablement séparés ou conjoints dans leur série.

L'attention volontaire peut rétablir l'ordre, et tantôt composer sur un plan plus régulier, plus conforme au modèle, tantôt abstraire ce qui se trouvait déjà composé par les habitudes ou la pente naturelle de l'imagination; mais dans ce nouveau travail, l'imagination sert encore comme auxiliaire, lorsqu'elle ne prend pas l'initiative. La volonté procède toujours dans un certain ordre successif ; chaque acte est distinct et séparé de l'autre, quoique leur succession puisse devenir indéfiniment rapide. Au contraire, plusieurs ébranlements passifs et spontanés de l'organe externe ou du sens intérieur, peuvent persister confusément ensemble. L'individu peut donc sentir ou imaginer plusieurs modifications à la fois, mais il n'en peut vouloir ou penser qu'une. C'est là ce qui constitue le temps mesuré dans l'existence aperçue, et non point dans l'existence sensitive.

C'est celui aussi que Diderot eût pu appeler matérialiste par excellence, et nous verrons bientôt qu'il peut l'être plus que le toucher même. 
Dans une perception de couleurs variées, ou dans l'image qui frappe, comme simple, l'attention ou la volonté même, qui conduit et manie le rayon visuel, survient et détache tour à tour plusieurs éléments, plusieurs nuances qui se fondaient dans une seule, lorsque le sens parcourt ou saisit à la fois tous les objets situés dans son champ, l'attention les lui fait parcourir lentement, le fixe tour à tour sur chaque objet ou sur chaque face du même.

C'est ainsi qu'elle le conduit à des compositions plus exactes, par des abstractions mêmes qui contrarient sa pente naturelle. Ainsi, cette puissance volontaire que nous appelons attention, et qui n'est point commandée par la vivacité des modes, quoiqu'elle s'attache uniquement à eux, sans se réfléchir au dedans, peut seule donner à ses travaux une base solide dans le temps; elle seule crée au sein des images, mobiles et fugitives, ces archétypes ou modèles fixes qui servent comme de signaux de reconnaissance, lient la modification d'un instant à celle d'un autre instant et les ramènent toutes à l'unité d'existence.

Mais encore un coup, ces travaux, ces produits, sont uniquement en représentation, l'attention qui abstrait tout, ne peut abstraire ses propres actes, et l'agent qui représente disparaît ou se cache sous la chose représentée. Il n'y a donc dans les modes qui se rapportent spécialement à l'exercice de la vue, ni abstractions réfléchies, ni idées simples de la réflexion. C'est ici que toutes les facultés et les opérations de l'être qui perçoit, peuvent être caractérisées et jugées en dehors, car elles ne sont, pour le sujet même, que ce qu'elles apparaissent au spectateur, et les deux points de vue dont nous avons parlé coïncident. C'est ici encore que le rapport de causalité s'intervertit nécessairement, et par suite des premières habitudes du sens. Dans la production des couleurs ou des figures visibles, l'individu sent qu'une force quelconque qui n'est pas lui, influence et prédomine la sienne. La partie même de la perception qui lui appartient réellement en propre, tend toujours davantage à lui échapper, et il n'aperçoit les modes visuels, ou ne s'aperçoit presque plus lui-même que comme effet d'une cause étrangère. Sa puissance n'est donc plus l'antécédent du rapport de causalité, elle finit même par ne plus $\mathrm{y}$ entrer en aucune manière ; et transportée tout à fait en dehors, la connexion des effets et des causes n'est que dans l'ordre de postériorité des impressions qui se suivent habituellement, et semblent se produire les unes les autres.

La véritable unité s'obscurcit aussi et disparaît presque dans les compositions imaginaires; le rapport constant à cette unité fondamentale, sans laquelle il n'y a point de pluralité ou de nombre, demeure inaperçu : tout est mode ou accident, rien n'est substance. La modification ou la couleur qui persiste devient comme un fond auquel les passagères s'attachent ; mais celleci passe et change à son tour, et il ne reste aucun point d'appui fixe dans l'existence. Qu'est-ce que cette existence même, là où il n'y a ni objet, ni sujet permanent? ces modes transitoires, ces fantômes qu'on appelle idées peuvent- 
ils être dits exister? Les idéalistes sceptiques, et ceux qui ont admis la doctrine de la sensation transformée, ont raisonné comme pourrait le faire une intelligence réduite au sens de la vue, et ce que nous venons de dire est propre à faire voir combien il est dangereux de généraliser trop promptement les observations prises d'un sens en particulier. C'est ainsi encore que je justifierai la méthode que j'ai adoptée. 


\section{Chapitre V}

Des fonctions du toucher. -

Analyse des facultés et des idées élémentaires qui s'y rapportent.

\section{I. - Fonctions passives et actives du toucher. -Analyse de ces dernières considérées hors d'un contact immédiat. Leur parallèle avec celles de la vue.}

\section{$\underline{\text { Retour à la table des matières }}$}

Supposons d'abord un aveugle, dont la main inflexible, ouverte, tendue en avant dans une position fixe, serait disposée de manière à recevoir les impressions des corps durs ou mous, chauds ou froids, polis ou rudes qui y seraient appliqués. Il me paraît évident que, dans cette hypothèse, la main ne constituerait point un sens particulier distinct des autres parties du corps que la peau recouvre, et ne pourrait être susceptible que de ces affections générales qui ne se rapportent point d'elles-mêmes au siège, ni à la cause, mais demeurent toujours plus ou moins confondues dans le sentiment fondamental et absolu d'une vie tout intérieure dont elles font partie.

A. Comment les fonctions du toucher actif pourraient s'exercer hors $d u$ contact. - Dans cet état, appliquons sur l'organe des corps de figure quelconque, ou, nous servant d'un compas de deux bâtons croisés, ou encore de cet instrument connu dans le dessin sous le nom de pantographe linéaire ${ }^{1}$, dessinons sur cette face sensible, telle figure que nous voudrons. Assurément,

Cet instrument à double branche est tel qu'on peut faire à la fois deux copies d'un même dessin, l'une des branches suivant exactement tous les mouvements que la main imprime à l'autre. 
ces impressions qui ne peuvent agir que comme excitatives dans tel degré que ce soit, ne se circonscriront point d'elles-mêmes, chacune en son lieu. Ces mouvements dont l'être sentant n'a point le module en lui, seront bien ignorés comme tels, et la copie que trace, sur la main passive, la branche du pantographe qui s'y applique, ne pourra être plus connue que le modèle exécuté par l'autre branche sous une main active et directrice. Nous n'avons point ici le premier élément de la connaissance, la première condition de la perceptibilité, pas plus pour ce qui se passe au-dedans de l'être, que pour ce qui est hors de lui.

Donnons maintenant à la main la locomobilité de masse seulement. Faisons encore abstraction de sa flexibilité naturelle et même de sa sensibilité qui ne fait rien à notre objet présent. Mais, admettons une volonté, une force agissante, qui commence à s'exercer hors de l'instinct, suivant ses conditions appropriées. Supposons que l'individu (aveugle), mouvant sa main en avant, rencontre l'extrémité d'un bâton, d'un levier appuyé par l'autre bout, sur un plan fixe, comme il est déjà supposé avoir conscience de sa propre force (moi) et du terme organique de son déploiement, il aura, par une suite nécessaire, la perception d'un contraste très marqué entre le sentiment qui correspond à l'exercice de cette force libre d'abord, puis arrêtée ou contrainte par l'obstacle. Dans cet effort contraint, il sera bien impossible que l'action née de la volonté de l'individu se confonde dans un sentiment unique et indivisible avec la résistance ou la force opposée qui réagit contre la sienne.

Le même fait de conscience comprendra donc ici l'aperception de la puissance moi, qui veut et commence un mouvement, et l'aperception ou l'idée relative d'une force aussi agissante (non moi), qui s'oppose directement à elle, et par conséquent n'est pas elle, ou est en dehors d'elle.

Je dis que la force réactive peut être conçue comme agissante, et sous ce rapport il y a lieu à une sorte d'égalité ou d'équation entre les deux forces opposées qui demeurent d'ailleurs numériquement et individuellement distinctes, comme le fait même de conscience le suppose.

Donnons à ceci une sorte de forme algébrique, soit, pour abréger, la force consciente moi $F$, la force extérieure opposée $\mathrm{F}^{\prime}$; la conception ou le sentiment relatif direct qu'a l'être moteur et voulant d'une force égale opposée à la sienne peut être exprimée par $F=F^{\prime}$. Le mode fondamental où la personne se trouve ainsi constituée, en rapport double d'action et de réaction à l'égard d'une puissance non moi, pourrait être figuré ainsi F : F (inversé). (On sent bien que je ne prétends pas mettre en formule le fait de conscience. Ce sont de simples abréviations que j'emploie au lieu de mots, mais je n'oublie point que les notions réfléchies ne se figurent point.) 
Maintenant, puisque les deux forces sont agissantes et égales sous ce rapport, nous pourrons changer, dans la dernière expression, l'ordre des termes, ou mettre indifféremment le conséquent à la place de l'antécédent, sans qu'il y ait rien de changé dans le rapport ou dans le même fait de conscience qu'il exprime. En effet, que l'être moteur agisse sur le plan solide par l'extrémité du levier contigu à sa main (supposée inflexible), ou que ce levier poussé par une force vive ${ }^{1}$, placée à l'autre bout, agisse sur la main qui oppose la résistance active, les principales circonstances du phénomène fondamental demeurent égales. Le moi s'aperçoit dans sa résistance volontaire comme dans l'action initiante qu'il détermine, et il perçoit une force étrangère dans l'action qui vient provoquer la sienne, comme dans la résistance qui l'attend ; c'est toujours même détermination, même vouloir, même rencontre directe de deux forces, dont l'une (celle qui est moi) se mouvant vers l'autre, ou étant rencontrée par elle, n'est constituée en rapport d'action à son égard, que dans un sentiment de passivité qui doit toujours précéder, suivre ou accompagner le déploiement de l'effort.

On voit bien ici que la connaissance d'une force étrangère au moi pourrait en effet se rapporter au sentiment d'une sorte de pression médiate de l'objet contre l'organe.

Mais ce sentiment même de pression suppose une action volontaire opposée sans laquelle il ne pourrait avoir lieu ; on voit surtout que la sensation passive du contact peut n'avoir aucune part au phénomène ; puisque dans l'hypothèse où nous sommes, la résistance du plan, ou la force vive qui agit par l'autre extrémité du levier, se manifeste directement comme opposée au moi et hors de lui, sans aucune sorte de contact.

Nous pourrions concevoir également dans la même hypothèse comment la force de résistance, qui constitue notre idée de corps substantiel, pourrait être séparée de toute perception de forme, de dimension et d'étendue même, car le levier pourrait ne toucher la main que par un point dont la sensibilité serait calleuse ou nulle, et l'action motrice n'en serait pas moins exercée et le résultat perçu, comme nous le verrons bientôt.

Observons enfin qu'il ne serait pas impossible que la vision active s'exerçât de la même manière, ou que la force des rayons lumineux réfléchis d'un plan solide et agissant sur l'œil comme le levier sur la main, rencontrât celle de l'être

\footnotetext{
C'est sans doute bien plus en nous heurtant, pour ainsi dire, contre les forces vives des êtres semblables à nous, qu'en opposant notre action à l'inertie des corps bruts que nous avons appris d'abord à connaître ou à sentir nos propres limites. C'est à peu près ainsi, ou par la rencontre mutuelle des parties de notre corps pressées les unes contre les autres par une même force vive (moi), que ces parties ont pu nettement se limiter et se circonscrire. Dans les deux cas il y a rapport double ou proportion, mais il fallait remonter d'abord jusqu'au rapport simple primitif.
} 
moteur appliquée à cet organe, ou fût rencontrée par elle de manière qu'il en résultât quelque sentiment relatif de pression ou de résistance lumineuse.

Supposons à présent que la main, exerçant sa propriété de flexion, embrasse et saisisse l'extrémité du levier auquel nous pouvons donner certaine dimension, forme, etc. Lorsque l'organe tend à se replier ou à se former sur le solide, c'est encore une détermination volontaire, arrêtée par la force antagoniste hors du moi, mais la pression étant immédiate, le rapport primitif et simple de l'action à la résistance se complique ici de plusieurs effets sensibles collatéraux dont nous aurons bientôt occasion de faire l'analyse. Ce qu'il importe d'observer dès à présent, c'est que dans le mode relatif de conscience $\mathrm{F}: \mathrm{F}$ (inversé), l'ordre des termes n'est plus susceptible d'interversion, comme si F' était une force vive ou médiatement perçue comme agissante. Ici tout n'est plus égal et réciproque : la main, qui presse le levier solide, en est bien pressée à son tour, mais c'est en tant que la volonté commence et continue l'effort; il n'y a plus de cas d'équilibre et $F$ ne pouvant concevoir $F^{\prime}$ comme une simple puissance égale et opposée, demeure seule antécédent potentiel du rapport; la notion fondamentale du corps extérieur n'est plus alors que celle de résistance ou force d'inertie, tandis que l'idée de force agissante ou de cause productive (exemplaire de toutes les causes ou puissances, qui pourront être de suite conçues au dehors) se trouve toute fondée sur le sentiment intérieur de la production de l'effort.

Si nous supposons maintenant que la main s'emparant tout à fait de l'extrémité du levier, s'en serve comme d'un instrument mobile pour parcourir les contours du plan solide et figuré situé à l'autre extrémité, l'individu ne pourrait-il pas acquérir par ce moyen et en tenant compte des mouvements, qu'il donne à son instrument, des différentes directions que doit prendre son bâton, pour suivre les contours du plan, ne pourrait-il pas acquérir ainsi, dis-je, après plusieurs expériences la perception des formes variables, comme modes d'une même résistance extérieure constante, forme qu'il semblerait imprimer lui-même, plutôt que recevoir, puisque l'idée sous laquelle il les conçoit n'est pas différente dans cette hypothèse, de l'attention qu'il donne à des mouvements dont il dispose, ou des déterminations actives qu'il en conserve par le souvenir. On voit bien alors que la notion de corps résistant et figuré serait tout à fait indépendante de toutes les autres qualités tactiles, dont nous faisons nous-mêmes si facilement abstraction ; le contact immédiat n'y aurait aucune part.

N'est-ce pas à peu près ainsi que l'âme, s'emparant des rayons qui viennent au-devant d'elle, les saisit, les dirige, comme la main de l'aveugle qui ne connaît d'abord que l'extrémité du bâton à qui elle s'applique ${ }^{1}$ ? Le premier

Dans l'hypothèse du Traité des sensations, la statue n'a d'abord connaissance aussi que de l'extrémité du bâton qu'elle tient; c'est là qu'elle rapporte toutes les sensations qu'il fait sur elle; elle ne sait donc pas qu'il est étendu, et par conséquent ne peut juger de la distance 
sens activé ne pourrait-il pas aussi apprendre de lui-même à mesurer l'écartement et la direction des rayons colorants et se faire ainsi quelque image colorée et figurée, qui flotterait au devant de lui sans se rattacher au plan solide ? (Necesse est consimili causa tactum visumque moveri) (Lucret).

Mais en revenant à notre aveugle, nous concevons bien qu'une fois qu'il se serait fait des idées de formes, par les mouvements qu'il a imprimés à son bâton, il serait capable de distinguer les figures qu'une main étrangère pourrait dessiner sur la sienne ${ }^{1}$, les impressions senties devenant, dans ce cas, comme les signes représentatifs des produits antérieurs de son activité. N'est-ce pas ainsi que les représentations visuelles s'accomplissent par suite des habitudes premières, sans aucun concours de l'activité qui les forma dans l'origine, et que les impressions passives de la lumière, ou les dessins en miniature qu'elle trace sur la rétine, sont uniquement les signes des créations de la vue et du toucher que la nature associe pour la circonscription active des formes et des figures ${ }^{2}$ ?

Les impressions sensibles de chacun de nos sens sont aussi, ou deviennent par l'habitude, des signes plus ou moins directs de la représentation objective ou de l'idée d'existence étrangère ; mais ces signes ont besoin d'être traduits pour conduire à la chose ou à la réalité signifiée. Le toucher actif entend immédiatement le langage de la nature extérieure; c'est lui qui atteint directement cette chose signifiée, cette force de résistance (et plus que non $m o i)$ que les sensations expriment ensuite, chacune à sa manière.

B. Conditions réelles du toucher actif. - Nos analyses nous ont découvert jusqu'ici, dans les sens, deux fonctions différentes, dont l'une, appropriée à une sensibilité ou une vie toute intérieure, est intimement unie à l'autre qui fait la vie de relation et la pensée. Il était peut-être assez délicat de bien distinguer leurs produits. Ici la fonction, qui se rapporte à la connaissance extérieure, est presque isolée ; ses produits s'offrent d'eux-mêmes comme distincts et séparés même de la sensation, et l'idéalisme fait de vains efforts pour s'aveugler sur le caractère essentiellement relatif des premiers, ou les ramener à la simplicité des modifications affectives qui se bornent à nous-mêmes.

Le toucher actif établit seul une communication directe entre l'être moteur et les autres existences, entre le sujet et le terme extérieur de l'effort, parce que c'est le premier organe avec lequel la force motrice, étant constituée d'abord en

des corps sur lesquels elle se porte. Il est bien clair pourtant que la statue devrait sentir une force qui résiste au bout de son bâton, et qu'en le dirigeant, elle pourrait acquérir plusieurs idées relatives au monde extérieur ; mais pour cela, il fallait lui supposer une force motrice, une volonté qui n'étant plus la sensation, aurait compliqué l'hypothèse et introduit deux éléments générateurs au lieu de l'unité systématique.

Je ne doute point que Saunderson n'eût aussi distingué un cercle d'une ellipse, tour à tour tracés légèrement sur sa main.

2 Ainsi le toucher, comme on l'a dit ailleurs, remplit, à l'égard de la vue, à laquelle il est associé, des fonctions semblables à celle de la voix dans son association à l'ouïe. 
rapport direct et simple d'action, puisse se constituer encore sous ce même rapport, avec les existences étrangères. Comme la connaissance et le double rapport de moi au corps propre et étranger se rapportent surtout à l'exercice d'une telle fonction motrice, la nature n'avait pas besoin d'établir, dans l'organe qui en est le siège, aucun appareil sensitif particulier, dont les dispositions ou les modes variés eussent altéré la constance du rapport qu'elle avait en vue. C'est donc uniquement vers les moyens d'une motilité plus parfaite, plus détaillée, et non vers ceux d'une affectibilité plus délicate et plus susceptible, qu'elle paraît bien ici avoir dirigé ses intentions.

Tous les sens externes ont chacun leur mode d'impressionnabilité particulière, approprié à la nature des agents ou fluides plus ou moins subtils de l'univers, qui, attirés vers eux comme par une sorte d'affinité élective, viennent les toucher, les exciter et peut-être se combiner avec leur propre substance ${ }^{1}$.

Ainsi l'œil diffère de l'ouïe non seulement par la conformation physique apparente, mais surtout par la nature intime du réseau nerveux qui y est le siège immédiat de l'impression lumineuse, tellement que si l'on supposait le nerf auditif ou la portion molle qui recouvre la lame spirale, substituée à la rétine, tout le reste demeurant égal d'ailleurs, il n'y aurait point de vision et vice versa.

Supposez au contraire telle partie que ce soit de la peau extérieure, grossière ou délicate, appliquée sur la main: les qualités tactiles ou les sensations de chaud, de froid, de poli, etc., seront modifiées, il est vrai, mais la fonction motrice essentielle ne changera point et ses produits immédiats resteront les mêmes, en tant qu'ils se rapportent à l'exercice actuel de la volonté et à la perception de ces qualités premières, toutes fondées sur celle d'une force de résistance extérieure ; c'est que de telles qualités ne s'adressent point réellement à aucune propriété affective et variable de notre organisation, mais qu'elles sont en rapport constant avec le mode unique de l'effort, déployé sur une seule espèce d'instruments homogènes ${ }^{2}$, dans toutes leurs parties, et par eux sur le terme étranger. Aussi, n'est-ce point seulement pour la main considérée comme le sens spécial du toucher, que ce terme étranger se manifeste dans sa propriété fondamentale ou ses qualités constitutives, mais encore pour tous les instruments de motilité qui peuvent s'y appliquer, le saisir

L'odorat et le goût en particulier sont doués de cette force chimique qui paraît faire la base de leurs fonctions.

2 Rappelons ici l'observation déjà faite par Bichat, c'est que les instruments propres de la motilité, ou les nerfs cérébraux qui activent cette fonction, sous l'influence de la volonté, n'offrent point les mêmes anomalies dans les propriétés physiques de couleur, de consistance, de grosseur, etc., qu'on remarque dans les nerfs, dont le concours ou l'expansion forment les organes exclusivement appropriés aux divers modes de sensibilité. (Ne peut-on pas conclure par analogie de la similitude des instruments à celle des fonctions et de leurs produits, et vice versa?) 
ou exercer sur lui une prise d'action ${ }^{1}$, comme par la locomotion générale du corps qui s'avance sous une impulsion volontaire, continuée et arrêtée par l'obstacle, ou détruite dans son effet, en tout ou en partie.

Le toucher est donc pleinement constitué par une fonction active et tous les modes associés ou collatéraux, qui ne dépendent pas immédiatement d'une telle fonction, ne se rapportent point à ce sens spécial. L'unité de force qui l'anime, la simplicité et l'uniformité des conditions de son exercice, enfin la nature de son objet propre, nous permettent de le considérer sous les formes les plus simples, qui constituent son essence, de prendre dans leur origine et suivre dans leurs progrès son essence, de prendre dans leur origine et suivre dans leurs progrès les opérations synthétiques sur lesquelles a pu se fonder son exercice actuel.

\section{II - Divers modes du toucher sous lesquels la même force de résistance se manifeste de plusieurs manières.}

$\underline{\text { Retour à la table des matières }}$

L'organe tactile au lieu d'être conformé comme la main de l'homme, pourrait se terminer comme un seul doigt entièrement recouvert d'un ongle aussi pointu qu'on puisse l'imaginer : ce doigt, locomobile à volonté, s'avançant contre un corps ou un plan solide quelconque donnerait à l'être moteur le perception d'une résistance ou force opposée, aussi distincte dans ce cas qu'elle l'est dans celui du levier poussé contre l'obstacle, dont nous parlions en premier lieu.

Cette résistance est ici naturellement abstraite ou séparée de toute conception d'étendue, de forme, et de tout ce qui est le plus intimement associé avec elle dans les habitudes actuelles de notre sens, étendu lui-même en surface, divisé, flexible et sensible dans tous les points, etc. Il n'y aurait d'abord dans le mode du toucher supposé que l'idée simple et absolument indivisible d'un point résistant. C'est sous cette idée que pourrait être uniquement conçue l'existence étrangère, par opposition à la forme moi, et l'on voit ici que la notion d'étendue n'entrerait pas aussi essentiellement dans l'idée fondamentale de corps, que les habitudes actuelles des sens ou de l'imagination nous portent invinciblement à l'admettre.

Le toucher s'exerce partout où le mouvement volontaire est possible. On touche avec le pli du coude, avec les jambes, les lèvres, la langue, etc. 
Si l'ongle aigu ne pouvait se mouvoir continûment, mais seulement par une suite d'actions répétées, et comme en sautant d'un point à un autre du plan solide, il y aurait idée de pluralité de points résistants, ou d'unités répétées qui ne sont encore que les résultats d'actes répétés de la même force contrainte, mais point encore d'idée d'étendue ou de composition par simultanéité ; l'unité se retrouverait et se reproduirait constamment égale à elle-même dans le sujet réfléchi et le terme objectif de l'effort; la conception réflective de moi (ou du sujet métaphysique) et l'idée représentative de l'objet mathématique se rapporteraient l'une à l'autre, et pourraient se servir mutuellement de type. Si l'organe avait la faculté de glisser continûment, sur le terme auquel il reste appliqué, l'opération se composerait un peu plus : ce sont bien, toujours des points résistants, mais qui se touchent, se continuent ${ }^{1}$, ou plutôt c'est le même qui flue en se reproduisant sous le mouvement non interrompu qu'une seule impulsion volontaire prolonge : ici le terme résistant paraîtrait de plus étendu en longueur, mais c'est encore la continuité de moi dans l'acte ou le mouvement prolongé qui détermine la continuité perçue de résistance, et la conception ou représentation extérieure de celle-ci se fonde nécessairement sur la réalité intérieure de l'autre ou la suppose ${ }^{2}$.

Si nous supposons maintenant que l'ongle aigu s'élargit de manière à pouvoir s'appliquer aux surfaces solides, mais sans pouvoir encore se replier sur les corps, ni embrasser leur capacité, ni se mouvoir successivement sur toutes leurs faces, en changeant de direction, il y aurait perception simultanée d'étendue en largeur, à laquelle la continuité du mouvement pourrait donner une extension indéfinie; mais il n'y aurait plus dans cette hypothèse de possibilité de se représenter directement l'unité résistante première, pas plus que d'imaginer la capacité ou l'étendue en profondeur.

Ainsi, l'objet réel du toucher et la force qui l'effectue restant les mêmes, chaque conformation possible de l'instrument de motilité aurait sa géométrie particulière, également assurée, également évidente. Dans tous les cas, ce qui

Leibnitz a supérieurement défini l'étendue : Resistentis continuatio.

Un philosophe (M. DEGERANDO, dans son Mémoire, couronné à l'Académie de Berlin), dont l'autorité est d'un grand poids, observe qu'il n'y aurait point de mouvements sentis ou perçus soit en nous-mêmes, soit en dehors, sans la mémoire, qui en conserve les impressions successives et les lie entre elles dans la même conscience. Il est certain que toute perception sensible, comme tout acte de l'intelligence, pouvant être, et étant réellement presque toujours composée de plusieurs impressions ou plusieurs actes successifs, qui coïncident en apparence dans le sens intérieur par leur extrême rapidité, il ne saurait y avoir lieu à la perception même la plus simple dans notre état actuel, s'il n'y avait pas continuité de moi ou réminiscence personnelle conservée dans la succession des termes ou modes élémentaires : mais cette réminiscence conservée dans la sensation d'un même mouvement, continu, doit être bien distinguée de la mémoire proprement dite, autant que la continuité de l'ébranlement nerveux, qui prolonge la même sensation doit être distinguée de la faculté qui rappelle, hors de toute occasion sensible. 
n'existe qu'en abstraction pour nous, qui ne concevons que l'étendue composée, serait dans chacune des hypothèses qui précèdent, la seule réalité existante ; mais il y aurait une notion fondamentale invariable et commune, tantôt directe, tantôt enveloppée plus ou moins, dans ces divers modes du toucher, c'est celle de résistance à l'effort, qui est la même et dans l'unité numérique rencontrée par l'ongle aigu, et dans la solidité linéaire, et dans tous les composés de l'étendue solide; d'où l'on peut conclure :

1. Que ce qui constitue relativement à nous l'essence propre de corps ou de matière, n'est pas autre chose que cette force de résistance, directement opposée à l'action que notre volonté détermine.

2. Que tous les doutes du scepticisme ne portent en aucune manière sur ce fond de notre idée de corps extérieurs mais sur certaines formes ou qualités qui, étant, en effet, toutes relatives à la conformation des sens ou à leurs dispositions, sont susceptibles d'une multitude de variétés.

3. Que nous ne sommes, enfin, ni plus ni moins certains de la réalité du terme étranger, que de celle du terme organique, et par suite, de l'existence même du sujet de l'effort ou du moi.

4. Que ce qui est pour nous (en effet) le dernier degré d'abstraction géométrique : les notions du point résistant du centre de gravité qui réunit en lui (et dans un véritable point) toutes les forces des masses, sont peut-être les plus rapprochées de l'absolue réalité des choses, ou du point de vue sous lequel une intelligence supérieure pourrait les contempler.

J'ai dit que dans cette origine de toute synthèse, le sujet métaphysique et l'objet mathématique, quoique conçus de deux manières différentes, peuvent néanmoins se servir réciproquement de signes symboliques. C'est à un tel point de vue que le génie le plus étonnant peut-être de tous les génies, Leibnitz, était arrivé en décomposant. C'est de là qu'il partit pour tout reconstruire ; c'est là qu'est le premier anneau de cette belle chaîne de rapports, la plus étendue peutêtre, que puisse embrasser ou concevoir une tête humaine.

Comment l'application de la même volonté motrice à divers modes de la résistance nous fait distinguer le corps qui nous appartient, des corps étrangers. - Si nous pouvions exister sans trouver de résistance invincible, ou si les termes étrangers de notre effort se proportionnaient à son déploiement, toutes les fois que la volonté s'applique à les mouvoir ou lutte contre leur inertie, la seule différence que nous mettrions entre ces corps et celui qui nous appartient en propre, serait en effet uniquement relative, dans ce cas, à la réplique du sentiment qui aurait lieu dans les uns, et non dans les autres. Mais comme c'est l'appropriation des modes à la volonté motrice qui fait aussi leur appropriation réelle au moi, nous ne serions peut-être pas éloignés de considérer ces corps 
comme faisant partie de nous-mêmes; notre volonté semblerait être l'âme de la nature extérieure environnante, comme elle l'est de cette portion de matière qui lui est soumise. Et, n'est-ce pas ainsi que les stoïciens purent s'élever, en effet, à la grande idée de l'âme du monde ? N'est-ce pas dans le sentiment de l'empire le plus étendu exercé sur eux-mêmes, qu'ils trouvaient le modèle d'une force intelligente suprême, à laquelle obéissent toutes les parties de ce grand tout que nous appelons l'univers?

L'inertie sentie dans les organes, que la volonté met expressément en jeu, suffirait pour constituer l'aperception dans un premier effort relatif, où le sujet et le terme (le moi et le non moi) sont (suivant l'expression des Kantistes) posés l'un à l'égard de l'autre. Mais ici, l'action ou la détermination motrice a son plein et entier effet, et le sujet ne fait, pour ainsi dire, que s'apercevoir luimême comme moteur. Là est la conscience du mouvement ou de l'acte volontaire, qui est différente de la sensation du mouvement.

Dans l'effort déployé contre un obstacle étranger, la détermination volontaire n'a pas tout son effet : il y a une partie de sa force qui se trouve arrêtée ou détruite. Or, encore un coup, ce qui arrête ou détruit instantanément cette force active n'est pas elle mais s'en distingue par un contraste bien prononcé. L'inertie matérielle ou physique se joignant à l'organique ou la remplaçant, détermine un effort plus intense ; le rapport primitif n'a point changé de nature, c'est toujours une volonté qui agit et un terme qui résiste plus ou moins ; mais les limites qui distinguent et séparent, jusqu'à un certain point l'antécédent et le conséquent, sont plus marquées, plus fixes, et ne sont plus sujettes à se confondre.

\section{III. - Association des deux fonctions dans la connaissance de diverses qualités qui constituent l'objet tangible.}

Retour à la table des matières

La connaissance ou le sentiment relatif des parties de notre corps, et la perception de résistance qui nous informe des existences étrangères, se rallient donc et coïncident dès l'origine avec l'exercice de la même fonction volontaire et le sentiment qui lui correspond. Cet exercice est-il libre ? C'est le moi et son corps. Est-il contraint ou arrêté ? C'est encore le moi et son corps, plus une résistance étrangère absolue. Mais dans la circonscription des formes, des figures, des situations, et dans la perception de l'objet total du toucher, tel qu'il 
parvient maintenant à notre connaissance, la même fonction, qui a servi de fondement exclusif au rapport simple du sujet à l'objet, n'entre plus que comme moyen nécessaire, il est vrai, dans une fonction mixte, et un ordre de rapports variés et compliqués, qui étendent et complètent la connaissance objective.

A. Rapport composé ou proportion qui sert de base à la connaissance des formes. - Les métaphysiciens qui sont partis de ce second ordre de rapports, sans remonter jusqu'à ses éléments, ne pouvaient, ce me semble, en bien assigner la nature ni embrasser tout ce qui s'y trouve renfermé.

Lorsque la volonté, par exemple, s'applique expressément à mouvoir un organe, celui-ci est connu directement dans le rapport de son inertie ou comme terme immédiat du déploiement de l'effort : il devient ainsi, par suite, un siège déterminé, auquel les impressions se réfèrent, où elles sont senties et alors seulement perçues; mais la connaissance objective de la forme particulière de cet organe, de sa situation ou sa distance à telle autre partie, ne résulte point de même immédiatement de l'exercice simple de la motilité : il faut de plus un double contraste et l'application successive d'une commune mesure, qui circonscrive les limites, il faut un instrument qui puisse se mouler sur toutes les formes, prendre toutes les situations, etc. Ici est la fonction propre du tact et de son sens spécial, qui étend la connaissance, complique et multiplie les rapports primitifs, mais ne les crée pas. La motilité entre aussi dans cette seconde fonction, comme moyen essentiel, mais sans constituer exclusivement la sensation tactile ; la main est connue d'abord comme tout autre organe mobile, par un rapport direct et simple, et comme terme de déploiement de la volonté. Mais quand ce terme mobile et sensible est appliqué à d'autres parties, ce n'est plus dans le rapport seul de l'organe à la force qui le met en jeu, mais dans celui de la partie touchée à l'instrument qui en circonscrit les limites, qu'est la perception alors composée.

Il en est absolument de même, quand la main s'applique à un corps étranger. Ce corps est connu d'abord dans la propriété fondamentale, qui le constitue terme résistant hors du moi : le rapport simple et direct résulte de l'exercice même de la motilité contrainte ; il demeurerait constamment égal, de quelque manière que l'organe tactile fût conformé, ou quel que fût le mode de son application à l'obstacle. Mais lorsque la main embrasse le solide, se moule sur lui, ou parcourt successivement chacune de ses faces, de ses arêtes, c'est encore le rapport de l'instrument mobile et sensible aux parties mesurées et circonscrites, et par suite les rapports de ces parties entre elles, qui servent de base à la perception ou au jugement des formes, des situations ou des distances : c'est ici qu'entre aussi la part essentielle de la sensibilité du tact. 
B. Part de la sensibilité dans celle connaissance. - Cette fonction contribue ${ }^{1}$ bien réellement d'abord à tracer les limites, qui séparent notre propre corps des corps étrangers par le contraste nécessaire qu'offre un sentiment redoublé, quand la main s'applique à une partie qui nous appartient, et simple ou sans réplique quand elle rencontre une partie étrangère.

En second lieu, dans la perception des formes, l'objet immédiatement connu comme résistant l'est de plus et dans plusieurs autres qualités accessoires simultanées qui, intéressant plus ou moins la fonction sensitive, se rapportent en même temps, soit à la main comme siège propre, soit à l'objet comme cause ou sujet étranger. Mais comme le jugement ou le rapport d'un mode quelconque à un terme, siège ou objet déterminé, suit toujours le déploiement de l'effort, en se composant ou s'abstrayant avec lui, et que la résistance organique peut être ici considérée comme nulle, eu égard à celle du terme extérieur, ce sera dans ce dernier surtout que devront être perçues en résultat, les modifications ou qualités tactiles, alors même qu'elles ne peuvent être senties que dans la main.

L'organe mobile et sensible presse l'obstacle, mais il en est pressé à son tour : il agit, mais il pâtit en même temps; il prend la forme du solide, mais il en reçoit l'empreinte : ce n'est pas la matière qui se moule sous son action, mais c'est surtout lui qui paraît se mouler sur la matière. Enfin, si ce sujet qui perçoit a l'initiative d'action qui est, pour lui, un moyen de connaissance, l'objet perçu, indépendant quant à l'être ou aux qualités constitutives, semble bien avoir toute la prépondérance de réaction.

Mais toutes les fois que la motilité n'entre ainsi dans la fonction perceptive que comme moyen, elle tend toujours à s'obscurcir sur les produits plus ou moins éloignés qu'elle active, et qui finissent par devenir buts, ou termes propres apparents du vouloir.

Cette observation est générale et suffit seule pour nous expliquer la rupture complète de l'équilibre de nos facultés d'attention (objective) et de réflexion, par suite l'aveuglement où nous sommes sur nos actes les plus intimes.

L'analyse des autres sens nous en a fourni assez d'exemples; l'exercice du toucher est soumis à la même loi, et ce que nous venons de dire de sa

Je dis contribue, parce que cette condition de sensibilité n'est point du tout exclusive, comme on l'a cru, d'après Condillac. Quand mes deux mains seraient entièrement paralysées pour le sentiment, il suffirait qu'elles pussent être mues l'une contre l'autre et se résister, pour que cette réplique d'effort ou de résistance les déterminât comme distinctes l'une de l'autre, et appartenant au même moi. Si l'une de ces mains seulement était insensible, celle qui conserverait la sensibilité pourrait la sentir comme étrangère sous ce rapport ; mais les deux efforts se rencontrant de nouveau, l'étrangeté disparaîtrait, et ce cas rentrerait dans le précédent. On voit bien ici le partage net des deux fonctions. 
subordination apparente aux causes d'impressions extérieures, comme ce que nous avons dit auparavant sur l'activité réelle qui lui est inhérente, nous conduit à assigner ce double caractère des facultés qui se rapportent originairement à cet exercice.

\section{IV. - Caractère de l'exercice des facultés ou opérations qui se rapportent à l'exercice du toucher actif.}

$\underline{\text { Retour à la table des matières }}$

$\mathrm{Si}$, comme le pensent aujourd'hui la plupart des philosophes, le toucher était le premier ou même le seul sens qui pût franchir le passage qui sépare le sentiment intérieur de nos propres modifications, de la perception ou connaissance relative des objets qui les occasionnent, lui seul aussi devrait examiner la pensée hors d'elle-même. En donnant à la représentation imaginaire cette base fixe et solide qui manque à l'exercice isolé de la vue, il compléterait cette sorte de réflexion spéculaire, où l'être pensant, qui se cherche et se mire dans un miroir presque toujours infidèle, s'éloigne de plus en plus de la réflexion concentrée, où il peut saisir ses propres formes.

Cependant, si ce sens fournit seul à la pensée le moyen de sortir d'ellemême, ou s'il l'entraîne irrésistiblement dans le monde extérieur, il lui donne aussi un point d'appui fixe et solide, d'où elle peut se réfléchir avec une nouvelle activité, et rentrer plus profondément dans son propre domaine.

Tout ce qui a été observé précédemment sur le caractère des fonctions du toucher nous conduit à reconnaître, dans l'exercice spécial de ce sens, un certain équilibre entre les deux éléments de la perception et les deux ordres de facultés qui s'y rapportent, équilibre que l'exercice isolé de la vue ne comporte point et que son concours devra souvent encore contribuer à rompre. Là, une représentation presque toute passive enveloppe le sentiment personnel ou la perception des actes rapides et légers qui persistent en résultat par la seule énergie de la cause extérieure. Ici la représentation ou plutôt l'intuition objective, activée nécessairement par la volonté, tourne au profit même de la réflexion et de la connaissance propre du sujet, qui se distingue dans ses limites, aperçoit mieux ce qui est lui par le contraste de ce qui ne l'est pas.

Lorsque la main s'applique au solide résistant pour en circonscrire les parties et en déterminer les formes, il y a, comme nous l'avons observé, un 
double rapport senti ou perçu; le premier, direct et simple, inhérent à l'exercice de la motilité arrêtée, constitue dans une même intuition le sujet de l'effort et le terme étranger qui existe comme force unique de résistance ; par là, dis-je, le moi devient capable de connaître ses propres limites et de se circonscrire. Placé entre deux sortes d'observations, l'une extérieure, l'autre intérieure, ce premier jugement simple appartient à la fois à toutes deux; elles s'y activent réciproquement l'une par l'autre. Un second rapport se fonde sur le concours nécessaire de deux fonctions, dont l'une, dépendant spécialement de la volonté, sert de moyen nécessaire à l'autre, qui se subordonne aussi en partie aux impressions de l'objet. Ce second rapport composé finit par être exclusivement perçu dans l'objet même ; les formes, la situation, les distances, etc., des parties s'y trouvent déterminées en résultat, indépendamment de tout acte ou mouvement aperçu comme venant du sujet. Là est aussi le domaine propre de l'attention.

Il est remarquable que le sens de la vue qui semble prendre une part si importante à la limitation des formes ou des figures, et remplace si promptement le toucher dans ce travail, demeure étranger au rapport simple de résistance, quoiqu'il fournisse des signes médiats et plus ou moins vrais ou illusoires aux autres qualités tactiles. Si toute force de résistance pouvait donc être séparée de ces dernières qualités ou de ce qu'on nomme en général les sensations de tact, ce dernier sens continuât-il à procéder par une suite de mouvements, qui ne feraient qu'effleurer légèrement les surfaces, il serait bientôt tout entier en intuition ou représentation spontanée, et l'on pourrait y appliquer tout ce que nous avons dit du caractère des opérations et des idées, qui se rapportent à l'exercice de la vue, où se trouve réalisée en effet la séparation que nous venons de supposer.

Mais autant que la volonté se déploie expressément contre la résistance étrangère, ou que le toucher, avant de glisser sur les surfaces, tend, pour ainsi dire, à les pénétrer; autant le sujet s'aperçoit dans le rapport de l'acte à son résultat objectif, et si l'attention, qui touche avec l'œil, entraîne l'individu loin de lui-même, la réflexion, s'appuyant sur un toucher plus approfondi, surtout plus lent et plus successif, retient l'être moteur rapproché de ses propres actes, et ne lui permet pas de se perdre de vue.

En comparant dans ces diverses circonstances l'exercice du toucher à celui des autres sens, on trouve que tout concourt à y maintenir cet équilibre des facultés dont nous avons parlé, et à faire prédominer dans l'origine celles qui se fondent sur l'aperception des actes, plutôt que celles qui l'excluent.

Ce sens est le seul qui aille chercher son objet dont l'action ne peut jamais le prévenir. La lumière, les sons externes et les odeurs viennent surprendre, heurter leurs organes; la matière tangible attend passivement l'application du 
sien; c'est un acte et non une impression qui la manifeste ${ }^{1}$. Si une détermination expresse active d'autres sens, le vouloir peut n'y être qu'instantané, et presque, tous les faits résultants se rapporter à l'impression, qui s'entretient par l'énergie de sa cause extérieure ou par une certaine vibralité propre, en différents degrés, à tous les organes affectibles. Dans le toucher, la perception ne peut pas plus se continuer que commencer hors d'une détermination volontaire, qui ne doit pas seulement donner la première impulsion, mais de plus l'entretenir par un effort prolongé, ou par une succession d'actes non interrompus.

La main ne peut embrasser chaque fois qu'une portion d'étendue égale à la sienne, et pourtant, il doit y avoir un ensemble de formes comprises sous une seule et même perspective tangible, il y a multiplicité de parties, variété et encore pluralité d'impressions et de qualités tactiles, senties par l'organe externe, et pourtant il doit y avoir unité et identité objectivement conçues dans le sens intérieur. Attribuerons-nous ici la coïncidence finale de plusieurs impressions successives, dans une seule résultante simultanée, à une disposition vibratoire qui conserve et réunit en un effet total les ébranlements organiques ? Mais ces ébranlements - quelle en est la force impulsive ? est-elle toute extérieure ? la matière tangible est-elle bien propre à les produire, et les fibres de la main à les recevoir, les conserver et les transmettre ? C'est-il, comme dans la vue, un fluide éthéré, irradié du dehors, qui monte l'organe à son ton et lui imprime ces déterminations persistantes qui, sous le nom d'imagination ou de mémoire, ne sont que la sensation même prolongée ? Enfin, lorsque plusieurs actes ou leurs résultats successifs sont réunis en quelque sorte sous un seul terme sommatoire, qui est la perception totale de l'objet même, l'identité et l'unicité attribuée à ce terme objectif ne peuvent-elles pas prendre leur type réel dans la puissance une et identique qui, ayant conservé le sentiment intérieur de ces actes, en transporte au dehors les résultats?

Tels sont les mobiles et les caractères propres aux opérations du toucher actif, trop souvent confondu avec les autres sens, dans certains points de vue d'idéalisme, comme dans des systèmes opposés. Tels doivent être aussi les caractères des facultés dérivées de l'exercice d'un sens, dont les procédés sont également appropriés à la réflexion même du principe qui détermine des actes, et à l'attention qui en saisit extérieurement les effets, sans être commandée ou attirée par la vivacité des impressions.

Telle est la cause aussi qui nous fait attribuer à l'objet uniquement tangible une inertie ou force morte, que nous n'attribuons pas également aux fluides, signes lumineux, sonores, etc., auxquels nous attribuons plutôt une activité propre, parce que ce sont eux qui viennent chercher ou provoquer nos sens. 


\section{V. - Opérations dérivées. Mémoire ou réminiscence personnelle et modale ou objective. Imagination.}

$\underline{\text { Retour à la table des matières }}$

Lorsque le toucher est appliqué de nouveau à un objet solide dont il ne peut avoir qu'activement embrassé et détaillé les formes, l'individu le reconnaît comme un ouvrage partiel de sa création; le sentiment du même être revit dans la représentation de ces modes actifs, sur lesquels il se fonda en premier lieu. La perception ne peut être renouvelée sans que la même volonté s'exerce, et la détermination de cette volonté même suppose le sentiment de son exercice antérieur. Il y a donc une réminiscence personnelle, inhérente aux perceptions du toucher, en tant qu'elles renferment essentiellement le rapport simple de la force à la résistance, puisque celle-ci ne peut pas se renouveler, sans que le même effort se déploie et par suite sans que le sujet reconnaisse sa propre identité.

La réminiscence objective ou modale n'est pas aussi infaillible; elle dépend davantage des habitudes ou de la répétition des actes qui, ayant acquis un certain degré de promptitude et de facilité, ne sont plus aperçus dans leur force déterminante, mais uniquement dans les résultats sensibles et composés. Ici, cette réminiscence suppose encore l'appropriation première des modes ou qualités tactiles à l'exercice actif du toucher.

Ainsi les formes, les situations de parties, les divers degrés de solidité ou de mollesse, de grandeur ou de volume, etc., sont reconnus dans l'identité même de l'objet résistant à qui elles se rapportent. Ce sont là autant de signes, sur lesquels peut se fonder le jugement de réminiscence, dont le caractère peut tenir ici également de la réflexion personnelle, et de l'attention modale. Mais il est d'autres modes qui, se trouvant exclusivement subordonnés à la fonction sensitive et hors des résultats de la puissance volontaire, quoique simultanés avec son exercice, ne sont susceptibles non plus par eux-mêmes d'aucune réminiscence dans le renouvellement de la même sensation tactile. Tels sont les divers degrés de chaud et de froid; de poli ou de rude, etc., qui ne restent les mêmes ni dans l'être sentant, ni dans la chose sentie et n'ont dans l'un ou dans l'autre aucun module fixe qui puisse les faire reconnaître. 
Nous voyons maintenant quelle sera, dans l'absence de l'objet tangible la partie de la perception qui s'appropriera au rappel volontaire ou à la mémoire proprement dite, car la faculté de reconnaître une modification présente, et celle d'en aviver intérieurement le souvenir, en l'absence de sa cause ou occasion première, ne peuvent différer que par le degré, et là, où celle-ci manque totalement, l'autre ne saurait se retrouver.

L'être actif a la faculté de rappeler les modes de la résistance, les formes des solides; il se crée de nouveau, pour ainsi dire, une seconde perception semblable à la première, tout ce qu'il avait mis du sien propre dans l'une revit avec une clarté parfaite dans l'autre; le terme étranger n'y est pas; les sensations tactiles n'y sont pas non plus; mais l'effort et la résistance organique subsistent : le mode actif, qui avait tout à la fois donné sa forme et reçu celle de l'objet, n'a pas perdu l'une et peut encore raviver l'empreinte de l'autre.

Sans doute il n'y a pas d'images des formes tangibles dans le cerveau de l'aveugle le plus exercé, comme il y en a des figures visibles dans le nôtre ; mais par cela même qu'il n'embrasse pas simultanément, comme nous, des perspectives étendues, il s'en fait des idées bien plus exactes et des notions surtout plus réfléchies; parce qu'il n'imagine pas les figures, il rappelle bien mieux les formes et les éléments de l'étendue.

La vue, comme l'imagination qui n'est encore qu'une sorte de vue plus intérieure, s'attache toujours aux effets d'ensemble : c'est la perspective qu'elle cherche et saisit. Nous nous représentons à la fois assez clairement des figures même compliquées, en nous aidant de la symétrie des signes, de la variété des couleurs, etc. ; mais quand il faut analyser sur le tableau imaginaire, compter ou dénombrer les parties élémentaires, puis les réunir de nouveau en une seule représentation, souvent la vue intérieure s'égare, se trouble et nous sommes réduits à substituer des signes aux images.

L'aveugle forcé de s'assujettir, dans la perception, aux procédés analytiques et à la marche essentiellement successive du toucher actif, a aussi une véritable mémoire des formes : il doit les rappeler en comptant en détail leurs éléments avec plus ou moins de rapidité. Pour lui l'analyse est toujours précédée de la synthèse, dans le sens comme dans le souvenir, et s'il ne se représente pas d'abord tant de choses à la fois, il est capable d'en retenir et d'en concevoir distinctement un bien plus grand nombre.

Ces observations sont confirmées par l'histoire de quelques fameux aveugles, tels que celui du Puysieux et du célèbre Saunderson ${ }^{1}$ qui possèdent

Le traité de mathématiques que nous a laissé Saunderson annonce une force de tête, et une métaphysique profonde qui le met au-dessus de bien des géomètres voyants. Il me paraît propre à confirmer ce que nous avons remarqué du caractère particulièrement réfléchi des perceptions de toucher séparées de celles de la vue. Peut-être les grands progrès dans la 
si éminemment le talent de rappeler et de combiner les formes comme les unités numériques.

\section{VI. - Caractère des idées qui se rapportent à l'exercice du toucher.}

\section{$\underline{\text { Retour à la table des matières }}$}

Le toucher, isolé de la vue, se rapproche bien davantage (même dans son exercice naturel) du véritable objet mathématique, qui n'existe pour nous qu'en abstraction. La géométrie de l'aveugle est une sorte d'arithmétique sensible, une combinaison de véritables unités ou points solides. Elle est plus près aussi de fondement de la source commune de toute science : de ce point commun où toute analyse aboutit, et d'où toute synthèse repart, où le physicien est conduit, en quelque sorte, à intellectualiser la matière, où le géomètre aussi rencontre le métaphysicien, où leurs conceptions d'unité, de force tendent à se modeler sur le même type.

Lorsqu'on dit que le toucher est le sens géométrique (il est entendu qu'on ne parle dans ce cas que du toucher actif), on exprime en un seul mot le caractère propre, comme l'inépuisable fécondité des idées dont il est la source. Le fond et la matière première de ces idées ne ressortent pas sans doute du sein même du sujet pensant ${ }^{1}$; mais on ne peut douter que l'être moteur, qui contribue à se créer en quelque sorte ce premier fond, en exerçant hors de lui son activité, ne l'étende ensuite indéfiniment par un exercice tout intérieur de la même activité plus développée; la base étendue et solide n'existe pour nous que dans le déploiement de l'effort; elle n'est mesurée et circonscrite que par des mouvements dont nous disposons. Ce modèle premier est donné par le sens qui

métaphysique et les mathématiques qui en sont une branche, s'allieraient-ils mieux avec la privation complète de ce dernier sens. Il est certain qu'il contrarie bien plus qu'il ne favorise tout ce qui tient à l'exercice particulier de la réflexion et sans se crever les yeux pour se connaître, on pourrait bien, je crois, être privé de leur usage et n'en concevoir ou n'en accomplir que mieux ce dessein, témoin Bonnet.

Dire que l'espace est une forme inhérente au sens intérieur, c'est poser cette notion primitive comme absolue, c'est dénaturer son caractère essentiel de relation; c'est bien aussi trancher le nœud sur la condition première du fait de conscience, et donner, en quelque sorte, un article de foi pour principe ou point de départ de toute philosophie. Si l'espace est une forme du sens intérieur, qu'avons-nous besoin de supposer un hors de moi existant, un noumène. (J'emprunte ce terme de l'ouvrage de M. Villers, où j'ai puisé presque tout ce que je connais du système de Kant. En adoptant un parti contraire, je n'en rends pas moins justice au véritable talent philosophique de cet auteur.) Ce principe est excellent pour justifier l'idéalisme. 
reçoit ou prend son empreinte ; mais bientôt l'entendement cessant d'imiter, crée lui-même ses modèles et se fait des archétypes, qu'il effectue ou réalise hors de lui, par des figures conçues ou tracées sur cette même base modifiable à son gré, et qui ne fait plus que fournir un appui et des signes aux créations de sa force active.

Les combinaisons infinies que le géomètre peut faire avec de l'étendue, des points, des unités numériques, ne sont point véritablement abstraites des impressions du toucher ni des perceptions directes, où l'on dit quelquefois (un peu vaguement je crois) qu'elles sont renfermées. Le mode de leur création actuelle prouve assez que ces idées ne se tirent point par abstraction des composés sensibles comme les idées des qualités que les métaphysiciens, ont appelées secondes; mais il faut se reporter à l'activité originaire du sens et à la manière dont il circonscrit son objet, pour concevoir le modèle naturel de ces sortes de créations ultérieures, qui, dans le développement des facultés, paraissent si spontanées et si indépendantes de toute impression du dehors.

Les idées dont il s'agit ne peuvent non plus être rangées dans la classe de celles que nous avons distinguées sous le titre d'abstractions réfléchies, ou encore d'idées simples de la réflexion (Voyez chapitre 3).

Dans l'exercice complet du toucher les modes de l'étendue solide s'abstraient les uns des autres par la succession des mouvements, pendant que le sujet conscient des actes, sur lesquels se fonde en résultat la connaissance objective, se fait les idées réfléchies de ceux-ci, ainsi que nous l'avons vu des autres sens actifs, mais les produits des deux facultés d'attention et de réflexion ne se transforment point réellement les uns dans les autres, et ce qui se conçoit ou se représente comme extérieur, dans les abstractions modales, diffère toujours de ce que l'être pensant aperçoit en lui-même, comme formant en quelque sorte son propre apanage ${ }^{1}$.

Il règne ici une analogie bien remarquable entre les notions premières du sens du toucher actif, telles que celles de force extérieure, de cause, d'unité, d'identité, de substance, conçues objectivement, et les mêmes idées simples prises de l'intime réflexion de nos actes : correspondance telle, que le jugement, soit qu'il s'applique aux résistances étrangères, soit qu'il se replie sur la nôtre propre, repose sur deux bases également fixes, transporte à l'une ou à

\footnotetext{
Ces deux sortes de conceptions si différentes se trouvent confondues dans le système de Kant sous le rapport commun d'origine, et cette confusion tient à celle des deux idées fondamentales d'espace et de temps, qui sont assimilées dans leur nature et considérées également comme deux formes pures inhérentes au sens intérieur, quoique l'une soit essentiellement objective tandis que l'autre est uniquement réflectible. On peut donc dire dans ce système, comme dans celui de Condillac: Soit que nous nous élevions jusqu'aux cieux, soit que nous descendions dans les abîmes, nous ne contemplons jamais que notre pensée.
} 
l'autre certains attributs fondamentaux et les affirme de sujets semblables ou analogues dans leur nature propre, lorsqu'ils se trouvent dépouillés par l'attention d'une part, et la réflexion de l'autre, de toutes les formes ou modifications accidentelles (Voyez le commencement de ce chapitre).

Comme ce dernier objet est d'une importance majeure en philosophie et que la manière dont nous considérons l'origine du jugement peut donner lieu à beaucoup de contestations, nous croyons devoir ajouter encore ici, quelques développements.

\section{VII. - Appendice sur les deux rapports simples d'existence personnelle et étrangère. différentes formes du jugement primitif et fondamental. Conséquences des principes contenus dans ce chapitre.}

$\underline{\text { Retour à la table des matières }}$

Pour remonter jusqu'à l'origine du jugement et percer jusqu'aux racines de cette faculté aperceptive, base de toute intelligence, il ne suffit pas de chercher comment un objet extérieur, à qui certaines modifications se rapportent comme qualités, peut être constitué pour le moi (supposé déjà préexistant dans ses propres modes intérieurement sentis ou aperçus); mais il faudrait savoir, avant tout, s'il n'y a pas une condition primitive de l'existence de cette personne individuelle identique, douée maintenant de la faculté d'apercevoir, de sentir ses actes et ses modes en les rapportant soit à elle-même, soit hors d'elle (car le fait de conscience emporte également ces deux cas). C'est ce qu'ont négligé d'observer les métaphysiciens, et notamment MM. Ancillon et Dégerando. Or, dans tous les deux également, la chose sentie, perçue, ou le conséquent du rapport n'est pas l'antécédent ou le moi. Tout ce qui a trait à ce second jugement d'existence étrangère a été savamment discuté et analysé ${ }^{1}$ avec beaucoup de profondeur; mais le jugement ou le rapport simple et primitif d'existence personnelle ne paraît pas avoir jamais guère occupé les philosophes. Ceux qui sont partis du système des idées ou formes innées, ont bien tranché le nœud de toute difficulté, mais les plus sages partisans d'une philosophie d'expérience ont tous présupposé, dès leur point de départ, un moi qui existe intérieurement ou pour lui-même, par cela seul qu'il y a dès l'origine

Dans le Traité des sensations d'abord, et ensuite dans les premiers Mémoires de M. DE TRACY, insérés parmi ceux de l'Institut National. 
quelque affection éprouvée dans l'organisation vivante. Les uns à la vérité n'ont admis cette connaissance personnelle que dans la circonscription des limites du moi et de l'objet, en considérant ainsi le fait primitif de conscience comme renfermant essentiellement ce rapport, ou cette double connaissance. Mais Condillac, ayant établi nettement, dans la sensation intérieure, la connaissance ou le sentiment d'individualité, comme divers jugements ou rapports entre de simples modifications simultanées ou successives, et ayant pour lui maintenant encore des autorités qui ajoutent à la sienne, il m'a paru important de rechercher :

$1^{\circ} \mathrm{Si}$, en poussant l'analyse aussi loin qu'elle doit aller, on pouvait supposer ainsi une personnalité et quelque rapport intérieur des modifications simples à un moi qui est dit les sentir, sans admettre de plus quelque condition autre qu'une affectibilité toute passive.

$2^{\circ}$ Si ce jugement simple d'existence personnelle ne pouvait pas, ne devait pas même être considéré comme ayant des conditions propres et constitutives qui pourraient s'accomplir indépendamment de celles qui fondent la connaissance étrangère, laquelle n'étant certainement pas antérieure à celle de moi, pourrait bien aussi ne pas avoir été simultanée avec elle, comme nous nous sommes attachés à le faire voir.

Maintenant, si je ne me suis pas beaucoup trompé, nous nous trouvons avoir fait un pas de plus, et après nous être placés d'abord dans l'hypothèse simple de Condillac ${ }^{1}$ nous trouvons ensuite un composé jusque dans ce fait de conscience (admis comme primitif) où le moi est censé n'exister pour lui-même que dans la relation à une force étrangère. Nous pensons qu'il y a un rapport plus simple et antérieur à celui-là : à plus forte raison sommes-nous forcés d'entrer en opposition sur ce point avec les philosophes qui, ayant égard à l'ordre des connaissances d'observation, partent du composé pour descendre au simple, et considèrent le premier de tous les jugements comme une analyse dont la sensation complexe serait l'antécédent, et la circonstance qui y est renfermée, le conséquent, tandis que suivant un ordre synthétique, relatif aux faits d'aperception intérieure, nous admettons un rapport simple primitif qui, fondant l'égoïté même, entre comme élément dans tous les autres rapports subséquents. Je n'ai plus rien à ajouter ici pour défendre ce point de vue et la méthode sur laquelle je la fonde: j'offre l'un et l'autre à des amis et investigateurs de la vérité, dont l'autorité sera toujours du plus grand poids à mes yeux.

Mais il est encore une question qui est la conséquence immédiate de cette première, et qui n'en diffère même pas au fond. Cette faculté fondamentale que nous nommons aperceptibilité, et que nous ne séparons pas du jugement, n'a-t-

1 Mais niant expressément l'identité qu'il établit entre l'aperception et la sensation. 
elle donc, pour ainsi dire, qu'une forme unique ? Les modes ou qualités qui se rapportent soit à un moi permanent, soit à quelque chose d'extérieur, dont nous sommes également forcés d'admettre l'existence durable, n'y sont-ils perçus que d'une seule manière ou sous la relation unique de la sensation totale à une de ces circonstances, du contenant au contenu ? Sur quel fondement reposent ces différentes sortes de connexions si profondément établies dans notre esprit entre le mode et la substance ${ }^{1}$, l'effet et la cause, la qualité et son objet, l'attribut et son sujet, etc. ? Tout cela est-il également réductible ou le rapport de compréhension se fonde-t-il sur une seule et même condition inhérente soit à l'exercice de nos sens, soit au terme extérieur de leur application?

Je me permettrai encore quelques observations à ce sujet. Le toucher actif, mettant seul l'individu en rapport direct avec une force de résistance étrangère, donne une cause extérieure à nos modes passifs qui, sentis ou perçus ainsi comme effets des corps, sont dits en être les qualités secondes; il donne aussi un objet fixe à ces modes fugitifs et variables, dont le caractère non affectif paraît être de se représenter ou de se projeter naturellement au-devant de leur organe, comme les couleurs ; ce sens, enfin, donne seul un sujet immédiat aux modes qu'il perçoit, d'après sa construction particulière indivisiblement de la force de résistance (quoique nous puissions concevoir une division de cette

1 Je dois craindre ici que l'on ne m'accuse de vouloir ressusciter des idées ou des dénominations vagues que nos philosophes excluent soigneusement et dont ils croient pouvoir se passer; mais j'observe que la notion de substance, qui malgré nous, entre dans toutes les formes de nos jugements et de laquelle celle de mode est corrélative, ne paraît vague et obscure qu'autant qu'on cherche à la mettre en représentation comme tout le reste, ou à pénétrer dans la nature absolue de la chose que l'on désigne sous ce nom. Or, en premier lieu, il est de l'essence de toutes les idées réfléchies de ne pouvoir se représenter ou se concevoir sous des images; et c'est l'oubli de ce principe qui a produit tant d'erreurs et de mécomptes parmi les métaphysiciens mêmes, qui ont été jusqu'à vouloir se représenter l'âme, par exemple, ou le moi pensant sous la figure d'un feu subtil, d'un petit corps éthéré, etc. En second lieu, nous sommes tellement constitués que nous ne saurions jamais sentir, ou percevoir, ou imaginer les véritables agents dans les sensations, Ainsi ce n'est point le fluide lumineux que nous voyons, mais les corps ou objets qui réfléchissent ou décomposent ses rayons : nous ne touchons pas la résistance, mais les formes sensibles et composées; la force motrice ne s'aperçoit non plus en elle-même, mais dans son application à l'organe résistant ou au terme mu... Les forces qui agissent sur nous et contre lesquelles notre force propre se déploie ne sont donc conçues que sous la relation nécessaire à certains effets produits ; nous ne pouvons sortir de ce cercle de rapports, ni en isoler les termes pour pénétrer dans leur nature individuelle. «L'Océan a ses profondeurs à l'épreuve de la sonde ; les sources des rivières, qui fertilisent de vastes contrées, se cachent dans le creux des roches escarpées. De même ces principes d'action qui produisent, animent et changent l'univers et nous-mêmes, ne nous présentant que certaines faces, nous dérobent éternellement leur fond et leur manière d'opérer. » Mais quoique ce soit surtout et même exclusivement à des effets ou conséquents, que notre pensée s'attache, elle n'en est pas moins invinciblement portée à supposer ou à affirmer l'existence des antécédents, si bien même que ce sont ces antécédents seuls, c'est-à-dire les forces ou causes fixes permanentes, et non point les effets ni les modes variables et transitoires, qui sont dits et jugés exister; car exister, c'est agir, et il n'y a que les forces ou substances qui agissent. 
dernière force perçue hors de tout autre mode attributif). (Voyez ce qui précède.)

Les modifications affectives, qu'éprouve l'individu dans un organe externe, en même temps qu'il perçoit ou juge la présence de quelque corps extérieur, ne sont point véritablement rapportées à ce dernier comme objet ni sujet, mais seulement comme cause ou force modifiante. C'est ici une association d'habitude de deux impressions ou plutôt d'une impression et d'un jugement qui diffèrent essentiellement par leur caractère. Hors de toute association l'une garderait, dans le sentiment absolu de l'existence, la propriété affective qui lui est inhérente : l'autre se fondant sur l'action réciproque de deux forces opposées, n'en conserverait pas moins en elle-même le caractère de relation qui lui est propre.

L'impression affective peut donc être, dans ce cas, aussi indépendante de la perception d'une résistance ou d'un jugement, que ce jugement l'est de la sensation. Il est des modes non affectifs, qui n'étant point non plus directement associés dans l'origine avec l'impression d'une force de résistance, s'y trouvent joints dans le temps et l'accompagnent toujours, quoiqu'ils varient sans cesse, pendant que cette force reste la même : de tels modes se rapportent au corps extérieur, non plus comme cause modifiante, mais comme objet, modifié luimême. Il est enfin des qualités que les métaphysiciens ont distinguées sous le nom de premières, qui n'ont pu être perçues que dans le déploiement de notre action propre et la réaction d'une force directement opposée. Ces qualités constitutives du corps ne s'y rapportent point comme à une cause modifiante, ni même comme à un objet modifié, mais comme attributs inséparables du sujet ou de la substance, telles sont les formes tangibles, la solidité, la dureté, la mobilité, qui constituent véritablement notre idée complexe de corps extérieur.

Le jugement qui affirme l'existence d'une cause extérieure active, capable de produire en nous certaines modifications par une influence quelconque, comme de s'opposer directement à notre effort, s'associe à la sensation, mais n'en fait point partie intégrante, n'est point basé sur elle.

Lorsque nous disons d'un corps qu'il est chaud ou froid, odorant ou savoureux, nous ne faisons que joindre à une affection actuelle l'idée de corps ou de la cause extérieure connue, d'après l'expérience, par des attributs qui lui sont propres ; mais les modes affectifs, qui ne peuvent jamais se rapporter qu'à nous-mêmes ou à une partie de notre organisation, n'entrent point réellement dans l'idée du corps extérieur, ne servent pas à la composer, et le verbe ne les 
affirme pas non plus comme circonstances ou attributs propres d'un sujet ou terme étranger ${ }^{1}$;

l'existence n'appartient point non plus à ces modes variables et s'ils étaient isolés comme ils le sont, hors des conditions propres et originelles du jugement, l'être sentant, qui les deviendrait, ne saurait les rapporter ni à aucune partie de lui-même comme siège, ni à aucun terme comme cause. Au contraire, le jugement qui affirme des corps les qualités ou attributs qui leur sont propres, comme étant inséparables de la force de résistance, les rapporte à ce corps comme siège, et à la force substantielle comme au propre sujet d'inhérence en qui ils sont réalisés hors de nous, indépendamment de la connaissance que nous en prenons; ce sont les effets immédiats ou directs par lesquels cette force étrangère peut uniquement se manifester à nous : elle existe dans les effets ou attributs (dans les phénomènes de l'étendue et des formes qui se réalisent en elle). Ici, les perceptions correspondantes à chacun des attributs ou modes de la résistance peuvent être dites, en quelque sorte, renfermées ou enveloppées dans le jugement fondamental qui établit pour nous une existence étrangère ; l'attention les fait ressortir ou les sépare successivement de l'idée totale de corps : ce sont autant de circonstances d'un même fait, autant de jugements partiels subordonnés au premier de tous, autant de rapports sentis, si l'on veut, entre un contenant et un contenu; mais il reste toujours vrai que le jugement fondamental n'en serait pas moins constitué, quand même la force simple de résistance serait isolée de tous ces modes circonstanciels que notre

Quoique notre langage usuel confonde presque toujours ces diverses attributions, il n'en fournit pas moins pourtant, dans certains cas, des moyens de distinction que la philosophie devrait, je crois, s'attacher à multiplier et à préciser davantage. Tout le monde dit, par exemple, qu'une fleur est odoriférante et non pas odorante: voilà une expression parfaitement appropriée au point de vue métaphysique qui ne diffère peut-être pas autant que l'on pense de celui du sens commun. Lorsque nous disons que l'objet est savoureux, il est bien clair que cet adjectif ne vient pas du verbe savourer; il en est de même des adjectifs sonore, lumineux, qui ne viennent d'aucun verbe d'action et ne peuvent s'y ramener. Il serait à souhaiter que tous les noms des modes passifs que nous rapportons, d'une part, aux objets comme cause, et à nous-mêmes, d'autre part, comme contribuant à les produire par notre action, que ces signes fussent aussi exacts que ceux d'odoriférant et d'odorant, ou formés sur le même modèle : ainsi l'on dirait de l'objet qu'il est savorifère, et de l'individu qu'il est savourant. Mais dans les modes complètement passifs, il n'y aurait pas lieu à la formation d'un participe correspondant à l'adjectif, les objets mêmes seraient dits calorifères, frigorifères, etc., mais point de participes analogues ${ }^{(1)}$. Si les langues s'étaient trouvées faites à peu près sur un tel plan, il n'y aurait pas eu lieu à cette prétendue découverte dont la philosophie s'est vantée, savoir que les qualités secondes, considérées comme nos propres sensations, ne sont pas dans les objets.

${ }^{(1)} \mathrm{Sic}$.

Assurément tous les hommes, sans être philosophes, entendent bien cela à peu près de la même manière ; mais c'est le langage qui a tort. Remarquons en passant, à cette occasion, que les adjectifs proprement dits ont une fonction bien différente de celle des participes, et qu'on ne saurait ramener les uns aux autres dans la théorie, sans y occasionner une sorte de confusion à peu près semblable à celle que nous avons remarquée dans la pratique. 
expérience ajoute, et que la forme actuelle de notre organisation ne permet pas d'en séparer.

Si les trois manières dont les modifications ou les qualités se rapportent à leurs causes, objets ou sujets, eussent été bien distinguées, une multitude de questions qui ont embarrassé les philosophes et les grammairiens (relativement aux fonctions du verbe en particulier) ne seraient peut-être jamais nées ; mais je ne puis ici qu'indiquer un point de vue qui m'entraînerait bien loin, et je dis en résumant : il est des affections simples en nous-mêmes qui sont séparées de tout jugement d'existence, de toute perception de rapport quelconque : l'effort que nous créons, et les modes affectifs qui en résultent immédiatement, d'une part ; l'opposition d'une force que nous sentons comme extérieure et les modes qui en sont inséparables, d'autre part, sont les seules bases fixes et les mobiles uniques du jugement qui est constitué dans ces modes, indépendamment de tout effet sensitif quelconque.

L'être sentant est affecté, et ne juge point naturellement que l'impression a son siège dans un organe, ou vient d'une cause étrangère. L'être actif juge, même sans sentir ou être affecté du dehors, que tel organe est le terme résistant de l'effort, ou le siège d'un mouvement qui se rapporte de lui-même à la cause moi qui le produit et le veut. Nous jugeons également, et nous ne sentons point l'existence d'une force extérieure qui réagit contre la nôtre, et produit, hors de nous ou sur nous, certains effets dont l'ensemble est appelé corps, et dont cette force est la substance et pour ainsi dire l'âme ou le principe d'unicité.

Il y a correspondance parfaite entre les modes actifs intérieurs rapportés directement au moi qui s'aperçoit en eux comme sujet et cause, et les qualités premières rapportées à la force extérieure comme à la cause qui les effectue ou au sujet qui les renferme : même parité entre les qualités secondes d'une part, et les affections internes de l'autre. Le jugement qui rapporte celles-là à une substance extérieure, et celles-ci à un terme organique, est également en dehors de ces impressions, et ne s'associe à celles-ci que par l'intermède d'une action antérieure ou concomitante déployée, ici, par la volonté seule, sur les organes résistants et impressionnables, là, par la force étrangère qui rencontre celle de la volonté et s'oppose à elle dans les mêmes organes. Dans ce dernier cas, l'analyse trouve un véritable composé, dans l'autre, elle ne trouve que le jugement pur et simple, dont elle s'attache uniquement à reconnaître les conditions originelles.

Les qualités secondes ne ressemblent à rien qui soit dans le corps ; ce sont des sensations ou des effets qui servent de signes à leurs causes. Mais est-il nécessaire qu'il y ait quelque similitude entre le signe et la chose signifiée, ou entre l'effet et sa cause, pour que l'un atteste la présence actuelle ou antérieure de l'autre ? Et quel parti raisonnable l'idéalisme pourrait-il tirer de ce prétendu défaut de ressemblance? Quant aux qualités premières, nous ne les sentons 
pas, mais nous jugeons qu'elles existent : reste à savoir si la force, par qui elles sont, ne nous est pas manifestée à l'égal de la nôtre ou notre existence même, et d'après tout ce qu'on nous dit, il ne saurait rester de doute. Ce qui peut être dit se ressembler en nous et hors de nous, ce sont les deux forces qui s'opposent l'une à l'autre, les deux substances sur qui portent toutes nos affirmations de modes ou de qualités, les deux causes actives enfin qui réalisent, séparément ou sans leur concours, les phénomènes objectifs et réfléchis des deux existences.

L'unité, la multiplicité, l'identité conservées dans la succession et la variété des modes, conviennent également aux deux forces, et celle que nous appelons substance corporelle n'est pas plus l'assemblage des qualités sensibles qui la manifestent, que le moi n'est l'assemblage de toutes les modifications affectives qui se succèdent dans le temps.

L'origine que le jugement (ou l'idée) d'existence étrangère prend dans les fonctions du toucher actif, et la manière dont il en dérive, me semble prouver que la connaissance ou le sentiment d'existence personnelle, et par suite toutes les facultés dont nous avons auparavant présenté l'analyse, ne sont pas absolument dépendantes de notre commerce avec le monde extérieur, ce qui revient à dire que la réflexion a son mobile propre d'activité intérieure, indépendant de tout effet de représentation objective. Les deux ordres de connaissances et de facultés demeurent donc toujours distincts quoique unis par les liens les plus étroits, et sans nous élever jusqu'aux cieux ni descendre dans les abîmes, nous pouvons contempler notre pensée.

Ce que nous venons de dire sur les opérations et les idées relatives au toucher confirme donc l'analyse des autres sens. Dans l'exercice particulier de ceux-ci, il pourrait y avoir une cause des modifications passives supposée, imaginée ou induite du contraste des modes perçus avec ou par l'action volontaire, et ensuite hors de cette action. Une telle cause serait conçue par privation, ou négation, elle serait $(-\mathrm{X})$; le toucher atteint directement, sinon à cette cause en elle-même, du moins aux produits positifs qui la représentent, conçue par opposition à la force volontaire, elle est $(-\mathrm{A})$ et quoique toujours $(-X)$ en elle-même, elle se détermine par des formes représentables, analogues seulement à notre manière de percevoir ou de connaître.

\section{Résumé général, tableau et projet d'une division et d'une énumération des facultés humaines}


L'analyse des sens et de leurs fonctions diverses ne doit pas être seulement, comme l'ont pensé quelques philosophes, une introduction nécessaire à l'histoire de la formation des idées, et de la génération des facultés intellectuelles ; elle devrait de plus embrasser, si elle était aussi exacte et aussi étendue que possible, cette formation et cette génération tout entière.

Les analyses qui précèdent peuvent, sinon confirmer entièrement ce principe, du moins servir de preuves justificatives à l'extension que nous prétendons lui donner ici.

Deux systèmes principaux paraissent partager depuis bien longtemps le vaste champ de la philosophie, le premier (et celui qui satisfait davantage au besoin des esprits contemplatifs) se fonde sur la distinction des deux ordres de facultés et de produits correspondants, l'un actif et regardé comme supérieur ou pur ; l'autre passif, dit inférieur ou empirique. Celui-là où le sujet pensant (inné à lui-même) fournit certaines formes et éléments de son propre fonds. Celui-ci, où les organes des sens et leurs objets fournissent toute la matière. Le premier système qui établit d'autres distinctions importantes, déduites de cette première, et souvent d'accord avec l'observation intérieure ou le témoignage du sens intime, prend néanmoins son point de départ hors de cette source; souvent il s'enveloppe de mystères, et récuse absolument dans certains cas ce même témoignage sur lequel il se fonde dans d'autres.

Le second système, approprié à ces esprits de lumière, qui ont acquis, surtout dans l'observation constante de la nature extérieure, l'habitude de ramener tout aux idées claires des sens, de ne jamais remonter au delà des effets, comme le droit de se confier à l'instrument d'une sorte d'analyse qui leur est familière, prétend faire tout ressortir d'une seule origine sensible et y ramener l'ensemble des idées et des opérations dont se forme l'intelligence humaine. On ne peut lui refuser l'avantage de la clarté du principe ; il a surtout celui d'une simplicité de méthode bien séduisante qui, rapportant à la même source les matériaux sensibles et les facultés ou opérations qui s'appliquent à les élaborer, assigne dans la nature même de ces premiers éléments les caractères constitutifs de ces facultés.

Il faut en convenir pourtant, l'unité systématique, sur laquelle se fonde cette dernière doctrine, paraît être plus dans l'expression que dans les faits mêmes. Les différences qui séparent ceux-ci dans notre sens intime, quoique déguisées en quelque sorte sous un petit nombre de titres, ou même sous un seul signe très général (la sensation) n'en sont pas moins reconnues au fond en réalité.

Quoique réduite à une seule classe de modes passifs, l'intelligence humaine n'en conserve pas moins des attributs d'activité ou de puissance individuelle, dont le langage même, établi sur une hypothèse contraire, est forcé de prendre l'empreinte ; et si l'hypothèse constitue d'une part toutes nos facultés en 
dépendance essentielle des impressions du dehors, elle n'en admet pas moins implicitement plusieurs actes qui, naissant uniquement au dedans de nousmêmes, ne peuvent que s'y réfléchir sans se représenter comme objets ou images, ni se sentir comme affections de la sensibilité.

Il ne me paraît pas qu'on ait entrevu ou cherché jusqu'ici aucun moyen de rapprochement ou de conciliation entre deux systèmes, moins opposés peutêtre encore dans leur point de départ, que dans l'ensemble des conséquences sur lesquelles se trouvent fondés leurs progrès ultérieurs.

Sans entrer dans ces oppositions de détails et de résultats, j'ai cru qu'en remontant au principe ou au point commun d'où ils commencent à diverger, il n'était pas impossible de trouver quelque voie d'une conciliation désirable ; j'ai cru qu'en faisant usage d'une sorte d'éclectisme, admettant : $1^{\circ}$ avec l'école de Leibnitz, comme faits (mais comme faits uniquement) d'observation intérieure deux ordres de facultés, l'un actif, l'autre passif; $2^{\circ}$ avec les disciples de Locke, deux classes d'idées, simples dans la réflexion, composées dans la sensation; $3^{\circ}$ avec Condillac et ses disciples enfin, une méthode naturelle de dérivation (tant des facultés que des sensations et des idées) d'une source mixte commune, les sens, et plus positivement l'exercice particulier de chaque sens, j'ai pensé, dis-je qu'il n'était pas impossible de réunir, jusqu'à un certain point, les avantages propres à chacun des systèmes, en évitant les écueils qui me paraissent se présenter lorsqu'on veut suivre exclusivement jusqu'au bout l'un d'eux en particulier.

Assurément l'exécution complète d'un plan aussi difficile, et si je ne me trompe assez neuf, demandait beaucoup plus de temps et de forces que je n'en ai à ma disposition. Aussi sera-ce l'essai d'une méthode particulière mixte, expressément applicable à la décomposition des facultés humaines (en donnant à ce dernier titre ${ }^{1}$ toute l'étendue qu'il peut avoir en métaphysique), plutôt qu'une analyse ou énumération parfaitement exacte de ces facultés, que je présente à mes juges dans le tableau suivant, comme résumé ou conséquence sommatoire de tout ce qui précède.

Voyez la première partie et la division psychologique de BACON. 


\section{Réflexions sur les résultats de la méthode de décomposition précédemment appliquée, projet d'analyse des mêmes facultés considérées dans un ordre supérieur d'exercice}

\section{$\underline{\text { Retour à la table des matières }}$}

Lorsque du terme où je me trouve arrivé, je mesure d'un coup d'œil la courte distance qui me sépare du point de départ, après une route si longue et si fatigante, j'éprouve, je l'avoue, un sentiment pénible et une sorte de timidité même, en songeant que le but où je vais m'arrêter n'est presque lui-même que le point de départ de plusieurs philosophes qui ont appliqué, avec le plus de succès, une analyse historique ou de déduction, à la connaissance des facultés de l'entendement humain.

Les sens forment ordinairement pour eux un chapitre assez court; ils prennent la sensation en général, ou toute manière (égale et uniforme par hypothèse) de sentir les impressions ou de percevoir passivement les résultats de l'action des objets, pour ce qu'elle est ou paraît être en effet, lorsqu'on la considère du sein même des habitudes de tous les sens, ou dans certains modes prédominants qui se rattachent à l'exercice de l'un d'eux en particulier, et tel mode spécifique d'un sens individuel (comme celui de la vue par exemple), comme servant de type aux autres ou formant le seul caractère réel de l'idée abstraite complexe exprimée par le terme sensation; on suppose l'analogie ou même l'identité de nature de tous les éléments qui se trouvent compris dans l'extension de ce terme, en se dispensant de la vérifier.

Le premier chapitre des sens ne peut donc être que bien court et les produits dérivés, par supposition, de cette source ou d'une autre plus mystérieuse, acquièrent plus d'importance et se trouvent caractérisés aussi par des signes plus clairs, plus faciles à saisir, dans un ordre d'idées et d'opérations d'autant plus familières et plus aisées à reconnaître qu'elles se trouvent placées plus loin de l'origine.

Je n'ai pas cru que les facultés intellectuelles, que les métaphysiciens ont établies ainsi sous des titres divers, pussent jamais être bien circonscrites dans leur domaine, leurs limites et la nature de leur fonction. Il m'a semblé que pour connaître le fleuve il fallait en sonder les profondeurs, et remonter le plus près 
possible de sa source, au lieu de le parcourir en surface, ou de le suivre dans son cours éloigné.

C'est sur ce chapitre premier et unique que je me suis arrêté ; c'est là que j'ai pensé qu'il fallait creuser. Est-ce avec quelque succès, ou mes peines sontelles perdues ? D'autres que moi en décideront.

Si la méthode que j'ai suivie ne m'a pas beaucoup trompé, il faut dire maintenant que tout ce qui est dans l'entendement, non seulement a été (fuit) dans le sens, mais de plus y est (est in sensu) comme instrument ou moyen indispensable d'opération réellement exercée et aperçue ; je dis tout, sans exception ${ }^{1}$; il n'y a que les virtualités ou formes abstraites qui ne soient pas dans le sens, elles ne sont aussi nulle part, hors du signe conventionnel qui fait tout leur être logique.

Les facultés élémentaires, qui auraient pu échapper sous un titre quelconque à mes analyses antérieures, devraient donc se retrouver, je crois, dans la même source creusée encore avec plus de profondeur et d'exactitude que je n'ai pu le faire. Ce serait ma faute de n'avoir su les y trouver ou les en déduire, mais si elles n'y étaient pas réellement, j'avoue que les omissions seraient irréparables pour moi maintenant, puisque je me suis interdit toutes voies pour chercher ailleurs de quoi les réparer (omnes aditus interclusi).

Sentir et agir, avoir conscience des modifications passives, apercevoir ses actes dans leur propre détermination, ou en percevoir simplement les résultats, et cela toujours dans un exercice actuel et positif de certains instruments organiques. soumis directement ou indirectement à une puissance de vouloir $(M o i)$, laquelle n'est point constituée elle-même dans sa propre réflexion hors de cet exercice, voilà, je crois, des facultés bien distinctes sui generis (élément générateur de tous les modes intellectuels ou opérations que l'on peut caractériser ensuite dans de nouveaux résultats composés sous une multitude de titres divers) ; mais voilà tout, telles sont du moins les bornes de ma vue...

Puis-je -croire néanmoins avoir fait un traité complet des facultés humaines? Je suis si loin d'une telle prétention que je m'estimerais heureux même d'avoir trouvé quelques-uns des matériaux solides qui doivent entrer dans les fondements de la science ; j'aurais satisfait ainsi en partie à la question proposée qui est bien fondamentale, toute en théorie et point en application, qui se rapporte à une science de nos facultés, dont la philosophie a senti les besoins, et point à celle de leur emploi ou de leur objet, si heureusement avancée de notre temps par le concours de tant de lumières.

La science des facultés n'est autre, selon moi, que celle des sens, en prenant ce mot dans l'étendue et la propriété de sa valeur. 
C'est dans ce point de vue et sous ce rapport fondamental que j'espère avoir répondu à la question. Mais si je m'étais encore trompé sur son véritable objet, si j'en avais mal interprété le sens, encore un coup, la faute serait irréparable, il ne serait plus temps de revenir sur mes pas, et en m'avançant je ne ferais que m'éloigner du véritable but.

Je devrais donc m'arrêter et terminer ici un travail qui, s'il n'est pas fini dès à présent, ne peut plus avoir de fin ni de commencement... et pourtant, après avoir creusé, il serait si agréable de pouvoir s'étendre en surface, de revenir jouir de la lumière dans un monde plus connu, d'y respirer plus librement (post descensum Averni revocare gradum et superas evadere ad auras) ! Sans doute, on regrette de laisser un travail souterrain sans que rien ne paraisse au dehors. Après avoir vu séparément et jusque dans les moindres détails les pièces éparses d'une machine, on aimerait à la voir en grand, dans son jeu et dans ses produits et (pour quitter la métaphore) après l'analyse, il est naturel de demander la synthèse qui lui sert de complément presque toujours nécessaire, et de preuve sans réplique.

Mais un travail d'application, d'une utilité peut-être plus directe, plus palpable, demanderait encore du temps et ce temps m'échappe, je me le suis ôté, il ne m'en reste plus que pour résumer dans une nouvelle sorte de tableau raccourci quelques matériaux, que j'ai à ma disposition comme les ayant rapportés du lieu bas d'où je sors, ou se continuant avec ceux que j'ai tirés de cette mine.

Voici donc à peu près le point de vue sous lequel je voudrais considérer l'application et faire une sorte de synthèse des opérations élémentaires dont j'ai tâché de reconnaître l'origine. 


\title{
Troisième section
}

\author{
D'une analyse des facultés humaines considérées dans leur exercice \\ général. L'association commune des sens entre eux et de leurs produits \\ composés à des signes artificiels ${ }^{1}$.
}

\section{Chapitre I}

Fondement de l'institution des signes. Second ordre de facultés intellectuelles. Parallélisme de cet ordre avec le premier tableau correspondant de leur division.

Retour à la table des matières

I. - Le premier et maintenant l'unique appui de cette faculté active d'aperception ou de réflexion, qui constitue éminemment selon moi l'être intelligent et pensant, me paraît être tout entier dans l'association des idées (ou des premiers produits d'une activité dont l'habitude a effacé le caractère), à des signes conventionnels ou artificiellement institués. C'est à l'emploi de ces derniers, et particulièrement à l'usage des sons articulés, pris dans cette nouvelle fonction de signes, que paraît commencer l'exercice de l'activité intellectuelle proprement dite. Hors de là, tout semble livré aux saillies d'une imagination sans frein et sans guide, point d'exercice disponible ou proprement volontaire des opérations de la pensée, et par suite, dans mes principes mêmes, point d'occasions, de moyens, ni presque de possibilité de réfléchir ou d'apercevoir ses actes, car ces deux conditions en effet sont réciproques et inséparablement liées. L'individu n'aperçoit réellement et d'une manière invariable (hors de l'habitude) que les actes dont il dispose ou qu'il veut, et il ne peut disposer que des actes ou modes aperceptibles, dans certaines conditions instrumentales dont nous avons parlé ${ }^{2}$.

\footnotetext{
Si la partie que j'ajoute ici n'était pas en quelque sorte de surérogation, j'aurais besoin de réclamer l'indulgence pour les abréviations actuelles comme pour les longueurs passées, et peut-être l'obtiendrais-je plus aisément sous le premier rapport.

2 Voyez le chapitre II, partie II.
} 
Il paraîtrait donc, qu'en se plaçant hors de toute institution de signe pour analyser les facultés humaines, on tombe dans l'inconvénient que j'ai voulu surtout écarter ou prévenir, celui qui consiste à voir, pour ainsi dire, les opérations de l'intelligence de dehors en dedans, et sans s'occuper de ce qu'est l'être pensant pour lui-même, de chercher seulement ce qu'il peut apparaître à un spectateur.

Ainsi l'on pourrait se croire autorisé à me rétorquer les arguments que j'ai pris la liberté d'élever contre une doctrine particulière qui se fonde presque entièrement sur ce point de vue extérieur.

Et, en effet, si l'on considérait uniquement la pensée comme art, je ne pourrais me défendre d'avoir encouru les mêmes reproches que j'ai osé adresser à des maîtres; et sous ce rapport il serait encore bien vrai qu'en laissant mon travail au point où je l'ai conduit, je terminerais justement l'analyse de la faculté de penser, là où cette faculté commence et où la philosophie s'en empare pour étudier les opérations et en régulariser la marche.

Mais avant l'art, est la nature, avant l'habitude et au sein des plus intimes habitudes, sont les facultés ou puissances fondées sur certaines conditions premières manifestées dans certains modes d'exercices qui les spécifient et caractérisent leur nature propre. Or, je dis que c'est jusque-là qu'on doit remonter pour connaître l'efficace des signes institués dans leur principe, et apercevoir le véritable mobile de cette institution. Je pense donc que nous nous trouvons maintenant assez avancés sur ce point fondamental, et que les principales difficultés relatives à la théorie de l'art sont levées, si nous avons bien suivi la théorie et les vraies formules de la nature.

Ainsi que le monde des êtres microscopiques disparaît à notre vue, ainsi que ces degrés mesurés, ces proportions prises avec tant d'exactitude par le géomètre sur un globe que nous trouvons si grand, disparaîtraient comme ce point globuleux au regard d'un être qui nous surpasserait comme nous surpassons l'insecte ; de même toutes ces divisions, tous ces degrés tracés dans une autre sorte de monde sensible, qui est bien microscopique pour notre œil intérieur, doivent se confondre et disparaître au regard étendu d'une intelligence développée qui, en possession de tous ses moyens d'exercice, planant également sur toutes les classes de modifications et d'idées, associant les produits de tous ses sens dans une communauté qu'il est devenu si difficile de rompre, transportant aux uns l'activité inhérente aux autres, ne saurait plus se reconnaître dans l'état primitif de germe, dont le tableau, même fidèle, lui serait offert, ni concevoir chacune de ses facultés séparées dans une source particulière.

Cependant, c'est dans ce monde des invisibles intellectuels que tout doit se trouver renfermé dans une sorte de germe préexistant à cet état, où la vue 
directe peut s'y appliquer ; c'est, ici qu'il ne se forme point de parties nouvelles, mais que les premières, voilées à nos sens par leur petitesse, ne font que se développer, s'étendre dans toutes leurs proportions sans trans-création ni métamorphose, d'où il suit que toutes les analyses infinitésimales, qui donnent les éléments, doivent se trouver applicables aux êtres complets et qu'on peut reconnaître encore ceux-ci dans leur premier état, en tenant note des circonstances qui ont concouru à les développer ou à étendre leurs parties sans en augmenter le nombre ${ }^{1}$.

D'où il suit encore (et pour ce qui nous concerne) que nous replaçant actuellement dans l'ordre naturel de l'observation, considérant les facultés humaines dans ce jeu et cette plénitude d'exercice où elles se signalent comme titres de divisions encyclopédiques, et s'érigent pour ainsi dire à elles-mêmes des monuments qui les font reconnaître, nous devrions retrouver les mêmes conditions, les mêmes caractères, les mêmes mobiles qui ont servi à tracer les divisions premières et élémentaires, en voyant ainsi amplifiés au regard simple ces mêmes éléments si subtils, découverts à une sorte de microscope, nous devrions, dis-je, pouvoir assigner, dans un ordre appelé supérieur, tout ce qui a été caractérisé dans l'inférieur, d'où il suivrait que ce supérieur n'est pas tel par sa nature, mais n'est que l'inférieur même qui a monté. C'est ce que nous allons voir peut-être en tenant compte, d'une circonstance bien importante, et d'un second élément dérivé dont nous n'avons pu parler jusqu'à présent et dont les partisans de l'ordre supérieur sont aussi trop souvent sujets à faire abstraction ; cette circonstance est celle de l'emploi des signes artificiels et du fondement de leur institution, dont nous allons maintenant traiter en peu de mots.

II. - Tout acte qui précède ou accompagne constamment une impression ou un mode quelconque, de telle manière que celui-ci soit senti ou perçu, comme en étant un résultat partiel ou absolu ${ }^{2}$, est, par là même, constitué signe volontaire de ce mode: signe intérieur qui seul transforme l'impression sensible en idée complète, en tant qu'il est aperçu sous la relation du moyen au but, de la cause à l'effet; c'est ainsi seulement qu'il devient disponible..., signe purement extérieur lorsqu'il est uniquement déterminé dans l'état instinctif par des affections vives qui excluent l'aperception et avec elle la disponibilité.

1 Mais qui est-ce qui peut ou qui veut bien s'armer du microscope de la réflexion ? Où sont les Lyonnets en métaphysique, et quand il y en aurait, comment seraient-ils traités par ceux qui ne voulant embrasser la nature intellectuelle que dans des points de vue généraux, n'aiment pas plus à s'informer en détail de ce qui peut être renfermé dans la première sensation, que les grands naturalistes ne s'amusent à compter les nerfs d'une chenille, ou à découvrir comment est pliée l'aile d'une mouche?

Je dis absolu ou total, lorsque le mouvement volontaire est moyen unique de l'impression; partiel, lorsque ce moyen concourt avec l'action d'un objet externe, comme dans l'exercice de la vue ou de l'ouie extérieure, d'un objet externe, comme dans l'exercice de la vue ou de l'ouïe extérieure, etc. 
Les signes ainsi considérés entrent donc déjà essentiellement dans cet exercice précoce des sens actifs, sur lequel se fonde la connaissance relative de moi (substantiel) et de mode interne organique ou d'objet étranger, exclu entièrement des fonctions sensitives et perceptives mêmes, où l'acte se trouve naturellement confondu dans le produit représentatif dont il n'est même pas toujours moyen nécessaire ; le signe se trouve institué par la nature même dans l'action complète du toucher et de la voix ; c'est de là qu'il ressort par une comparaison rapide mais assurée, qui s'établit entre la détermination même d'une force motrice et son résultat disponible, aperçu ou senti comme tel. Là est le rapport simple qui peut précéder le composé et aussi le modèle de toute association ultérieure active, qui, par une seconde institution du signe, va donner un double appui à la réflexion, une double prise à la volonté et deux moyens à la fin.

C'est assurément franchir un grand intervalle et s'épargner aussi un travail de réflexion assez difficile, que de fonder d'abord tout le langage sur la communication extérieure et sympathique des sentiments et des idées, sur l'imitation réciproque de deux êtres qui se trouvant tout d'un coup à l'unisson de pensée, comme d'organisation et de besoin, sont déjà supposés des personnes douées d'une certaine mesure d'intelligence. Ne faudrait-il pas savoir avant tout, comment elles sont arrivées jusque-là ; si elles ont pu y parvenir sans le secours de signes intérieurs déjà peut-être institués par elles, les mêmes dont elles vont faire usage pour transmettre au dehors les idées complètes, qui s'en trouvent revêtues?

Le langage institué ne crée point à l'individu des facultés nouvelles ${ }^{1}$; son emploi suppose bien plutôt le préétablissement des conditions particulières et l'existence d'une faculté vraiment supérieure à celle d'être affecté et de sentir. Mais cet emploi habituel d'un langage perfectionné réagit si puissamment, il est vrai, sur la faculté même institutrice, modifie, étend et compose d'une manière si remarquable le jeu de toutes les opérations intellectuelles dérivées, qu'en se plaçant hors de la nature et cherchant l'origine de la pensée comme art, c'est vraiment remonter à la source, que de tout rattacher à cette première institution artificielle du signe. Resterait à savoir si l'on peut bien en posséder

\footnotetext{
La seule différence, disent quelques philosophes, qu'il y ait entre les animaux et nous, c'est qu'ils n'ont pas nos signes; mais pourquoi n'ont-ils pas nos signes ? Ce ne peut être uniquement et d'abord, parce qu'ils sont privés de la faculté de remarquer une sensation particulière renfermée dans un autre, ou en étant une circonstance (c'est-à-dire de sentir en jugeant, ou plutôt de juger en sentant); car je crois bien que cette faculté originelle d'analyse suppose déjà elle-même quelque emploi des signes institués ; mais c'est la faculté de former le premier apport simple d'existence ou l'aperception de l'acte voulu, distingué de son résultat, qui leur manque, si je ne me trompe, parce que la manière dont tous leurs mouvements en général sont déterminés et effectués par les actions sympathiques en exclut le sentiment propre, et par suite exclut la possibilité d'un vouloir plus positif, qui attache une signification à ses mouvements et les institue signes.
} 
ainsi la théorie tout entière ; et si (pour me servir d'un exemple trivial), il ne faut pas savoir comment on marche, avant de s'informer comment on danse.

Les fonctions du toucher composent déjà avec celles de la voix, sous l'influence de la même volonté motrice, et indépendamment de toute communication artificielle, un système de signes et une sorte de langue naturelle, où se trouvent exprimés, savoir : dans les premiers signes du toucher, tout le fond réel de la nature extérieure ; et dans ceux de la voix, un véritable langage intérieur où les modifications les plus intimes de l'être sentant et pensant trouvent un signe d'annotation approprié qui les distingue, les double ou les réfléchit vers leur source ; il ne s'agit plus que d'étendre ces fonctions suivant le plan indiqué par la nature.

Or, sous la loi des habitudes et des affections (en supposant ces deux mobiles identifiés dans leur source ou le passage immédiat de l'un à l'autre, comme il arrive probablement dans l'état simple d'animalité), je dis encore que l'individu ne pourrait point ainsi ajouter à la nature ou imprimer à sa pensée quelque direction analogue aux résultats directs du premier exercice de son activité ; il faut qu'il lui reste une puissance d'agir et d'apercevoir ; mais cette puissance ne reste que dans le rapport de l'aperception à certains modes, produits d'une activité motrice qui sont en défaut dans l'instinct pur, et dont cette cause aveugle, non plus que l'habitude ne saurait diriger, obscurcir ou aveugler entièrement les produits dans la suite; tels sont les actes qui imposent les deux langues mères de la nature. L'être actif et intelligent les crée en tout ou en partie, les parle, les entend, les applique, et par là-même s'institue être pensant. Encore un pas, un second acte de la même puissance, un nouvel exercice de la même réflexion, et le langage artificiel se trouve complètement institué, et avec lui la pensée supérieure. Ce n'est point une nouvelle carrière qui commence mais un nouveau progrès dans celle qui se trouve ouverte par la nature à la perfectibilité de l'être capable de s'élever d'abord à la hauteur du signe intellectuel.

Nous n'avons plus besoin maintenant que d'indiquer les circonstances et les résultats directs de cette institution secondaire pour en voir ressortir complètement un second ordre d'opérations ou de facultés dérivées.

III. - La condition, qui donne au signe volontaire sa première valeur disponible, consiste toujours dans un certain équilibre de deux facultés ou modes d'exercice de la même puissance, distingués dans leur caractère originel sous les noms propres de réflexion et d'attention. Les conditions de cet équilibre peuvent se trouver naturellement établies dans l'exercice particulier d'un sens prééminent en cela sur tous les autres, mais elles ne sont pas toujours également remplies dans l'exercice même individuel de ce sens, encore moins dans son association avec d'autres qui les excluent. 
La détermination active, par exemple, qui prend l'initiative et doit conserver la prédominance dans les fonctions propres du toucher, peut s'effectuer de telle manière que l'effort presque entier s'absorbe, pour ainsi dire, dans l'inertie organique du sens encore obtus et peu exercé. Les formes du solide demeurent alors confondues dans la résistance totale ; rien ne se lie dans l'aperception, ni par conséquent dans la mémoire, et la détermination ou le rappel des mêmes mouvements, demeure à vide et sans objet. Au contraire, lorsque ces mouvements trop faciles sont devenus extrêmement rapides et légers, la perception de formes ou de figures est tout entière dans le résultat sensible ou objectif qui appelle au dehors toute l'attention du sens externe ; le rappel alors n'a plus de mobile, ou la volonté plus de prise, sur l'image dont la reproduction ou représentation paraît être devenue purement spontanée.

Les mêmes lois doivent présider encore à la formation d'un second ordre de surcomposés, car ce sont toujours mêmes moyens, mêmes conditions, même puissance.

L'association régulière des sons articulés à des modes ou idées quelconques ne peut se fonder également que sur un juste équilibre de l'aperception inhérente à l'acte vocal et de l'attention objective qui doit proportionnellement s'attacher au second élément associable de l'idée ou du produit intellectuel surcomposé ; c'est comme dans la part que prend le toucher à la perception du composé direct tangible et visible (necesse est consimili causa moveri). Si la réflexion se concentre avec le vouloir sur le moyen immédiatement disponible, le but ou le terme immédiat échappe ; le son trop laborieusement articulé, par exemple, demeure seul révocable, et l'image ou le mode associé reste loin du souvenir: que si c'est l'image dont la vivacité absorbe toute l'attention objective, la détermination du signe oral, ayant à peine effleuré la conscience, sera hors de la sphère du rappel ; la volonté n'aura nulle prise sur le seul des éléments de l'idée, qui put la mettre tout entière à sa disposition.

Mais ces conditions d'équilibre qui fondent une association régulière $(\ldots)^{1}$, l'exercice parfait de nouvelles facultés, trouvent souvent des limites ou des obstacles insurmontables dans la nature ou le caractère même des éléments hétérogènes à associer.

Le signe, par son titre et sa fonction même, ne peut être que constant, égal et disponible. Les éléments associables au contraire sont divers et de toute nature. Les modes actifs sont seuls homogènes entre eux. Dans les composés qui s'en forment, chacun peut jouer tour à tour le rôle de signe, quoique d'une manière plus ou moins parfaite ou assurée. Les termes, par exemple, destinés à représenter des figures ou des idées géométriques, sont infaillibles et constants dans leur propriété significative ; le composé réunit le caractère de clarté et de

Mot omis. 
disponibilité propre à chacun des éléments; la représentation objective y acquiert plus de netteté ; l'aperception est doublée dans le signe ou l'acte qui en détermine le rappel.

Les idées simples, ou modes directement représentatifs, prennent bien aussi dans l'institution du signe volontaire, qui leur est ajouté, un caractère de réflexion qu'ils n'avaient pas, mais ici la fin est telle, qu'elle marchera encore souvent avant le moyen, ou que l'image entraînera dans sa spontanéité naturelle le signe destiné à le modérer ou l'approprier à une puissance régulatrice. L'imagination prévenant ou entraînant quelquefois cette puissance pourra même la conduire dans certains cas, au hasard, plus heureusement qu'elle n'aurait pu le faire elle-même avec intention ou choix de moyens.

Enfin, le second élément d'association peut être d'une nature si hétérogène à celle du signe que l'activité intellectuelle ne puisse s'y appliquer par ce nouveau moyen artificiel, et que, dans les composés mixtes et toujours irréguliers qui s'en forment, le mode affectif signifié demeure toujours hors du vouloir ou l'absorbe ${ }^{1}$.

À ces diverses formes d'association correspondent autant de caractères propres à spécifier les facultés que nous avons à examiner encore dans un autre ordre, non de nature, mais d'exercice et d'application.

Considérant, en effet, le point de ralliement commun que prennent dans le centre, d'où la volonté irradie son action, les modes et les idées qui se trouvent associés à des signes disponibles, suivant les différentes manières qui viennent d'être indiquées, nous sommes conduits à rapporter les différentes opérations de la pensée, prise dans son exercice général, à autant de fonctions de ce centre qui correspondent parallèlement, et chacune à chacune, à tel ordre de fonctions (sensitives, perceptives, aperceptives ou réfléchies), attribuées auparavant à tel sens externe particulier, comme à sa source naturelle, en sorte que les mêmes opérations qui forment directement ce qu'on appelle en général (et d'une manière trop vague) les idées sensibles, se trouveront appliquées par le moyen des signes à la formation ultérieure, ou à l'élaboration des produits intellectuels de tout genre, connus sous divers titres, auxquels la métaphysique s'attache spécialement, en considérant ces produits bien loin de leur origine, ou ne les ramenant, pour ainsi dire, qu'en masse à la sensation. Essayons de montrer le parallélisme.

1. Et d'abord les images simples, produits spontanés de la réaction sympathique cérébrale dans l'instinct ou les passions naturelles, peuvent être considérées dans leur siège, centre particulier, comme les affections directes

Je ne dois pas insister ici sur les détails qui m'entraîneraient trop loin et qui se trouvent exposés ailleurs. Voyez en particulier le Mémoire sur l'habitude, déjà cité. 
dans les organes internes ou externes. Elles sont en dehors du siège de l'intelligence, et ne reçoivent pas plus le frein du signe que les impressions directes correspondantes ne reçoivent l'influence de la motilité volontaire. Observons aussi que toutes les affections immédiates, dont nous parlons, se caractérisant dans le physique par certains signes extérieurs appropriés, n'ont point de termes qui leur correspondent dans la langue des idées, à la hauteur desquelles leur confusion absolue dans le sens, leur nullité totale dans le souvenir, ne leur permet en aucune manière de s'élever; nous ne pouvons nommer sensations et encore d'une manière bien vague, que les modes composés du premier ordre, qui se trouvent déjà unis à une première forme intellectuelle de personnalité ${ }^{1}$.

Les signes, associés ainsi aux modes directement sentis, forment avec eux un premier ordre de surcomposés intellectuels qu'on pourrait appeler avec Locke (mais dans un point de vue plus analytique) idées de sensation. Ces idées renferment ou motivent dans le signe associé une certaine espèce d'opérations, que nous résumons, dans le tableau ci-après, sous le titre d'ordre passif intellectuel, première classe de composés de cet ordre (voyez ci-après).

2. Les perceptions ou images associées aux signes volontaires dans la prédominance de l'attention fixée par le résultat sensible de l'acte sur l'aperception de cet acte même, conservent dans le centre commun un caractère de spontanéité analogue à celui des représentations directes du sens. Toutes les opérations intellectuelles, qui se rattachent aux signes associés de cette manière, ou à de tels éléments, ont pour type les fonctions de la vue, et l'ordre de facultés immédiatement dérivées de cette source. Nous sommes autorisés à leur donner dans le tableau un rang parallèle à celui qui a été compris déjà sous le titre d'ordre actif, première classe des composés perceptifs. Nous ajouterons seulement à ce titre, comme au précédent, l'épithète d'intellectuel qui exprime, non des produits d'une nature absolument différente, mais un caractère d'activité supérieure que le signe rattache à l'image; c'est ainsi que la réminiscence devient plus assurée dans ce dernier ordre de composés, comme la forme personnelle dans celui dont nous venons de parler.

3. Dans les associations régulièrement formées par un juste équilibre de réflexion et d'attention, qui embrassent deux éléments ou modes actifs homogènes, les produits composés de toutes les opérations qui les élaborent conservent dans l'organe intellectuel le caractère de l'aperception objective, directe dans le sens externe; c'est l'exercice du toucher, et encore toutes les facultés immédiatement comprises dans cet exercice, qui leur servent de fondement et de type; nous trouvons donc ici matière à une seconde classe de surcomposés de l'ordre actif intellectuel, parallèle et correspondant à la même classe de l'ordre sensible, dans le premier tableau.

Voyez le tableau 1. Ordre de composés sensibles. 
4. Enfin, lorsque l'élément associé est uniquement de nature réflectible, analogue à celle du signe oral, ce sont deux actes réfléchis qui s'ajoutent l'un à l'autre, et le produit surcomposé de cette association est dans la pensée, comme la détermination vocale et auditive est à la fois dans le sens direct et répétiteur de l'ouïe et de la voix, qui sert aussi de mobile et de type à toutes les opérations ou idées de cet ordre ; il correspondra donc parfaitement à celui qui renferme la troisième classe de facultés considérées dans l'ordre du tableau suivant. Je résumerai et figurerai ainsi ces parallèles en donnant à chacun quelques développements utiles dans les chapitres suivants.

Base et projet d'une division des facultés, considérées dans les modes généraux d'exercice de la pensée ou leur complément d'activité intellectuelle, c'est-à-dire dans l'association commune des sens (ou facultés élémentaires), les uns aux autres sous l'influence des signes volontaires conventionnels.

\section{$\underline{\text { Retour à la table des matières }}$}

La distinction et trop souvent la séparation complète établies par les métaphysiciens entre deux ordres de facultés appelés, l'un intellectuel, l'autre sensible, se fondent sur l'hypothèse d'une dépendance absolue, où les sens et leurs premiers produits immédiats sont constitués à l'égard des impressions des objets extérieurs, dépendance qui ne peut plus avoir lieu quand, ces objets n'agissant plus, les idées restent ou certaines opérations s'accomplissent, etc. De là le motif d'une division ; mais en ayant égard aux fonctions proprement actives des sens, et à la part essentielle que prend la volonté dans la formation des premières idées dites sensibles, comme à deux sortes d'éléments de ces mêmes idées réputées simples; observant de plus, que l'institution des signes articulés a pour objet essentiel de fonder la production ou le rappel de toutes sortes d'idées et de modes sur l'exercice constamment disponible d'un sens tel que la voix et l'ouïe, on ne peut plus faire de séparation entre deux ordres (intellectuel et sensible) de facultés. Toute la distinction à établir, comme tout le changement à faire dans les classes qui forment le tableau précédent, pour qu'ils représentent l'ensemble des opérations élémentaires et constitutives de l'intelligence humaine dans la nature et dans l'art, consistent à tenir note des résultats d'un nouvel élément actif qui, étant introduit dans ces classes, compose les simples et surcompose les composés. De là un nouveau tableau qui ne peut comprendre que les éléments du premier, élevé seulement d'un degré dans l'échelle de l'activité intellectuelle, tout en conservant un parallélisme absolu. 


\title{
Chapitre II
}

\section{Ordre passif intellectuel}

\author{
Première classe de composés de cet ordre. \\ De l'imagination sensitive. \\ Quelques vues sur les passions et les sentiments moraux.
}

\section{$\underline{\text { Retour à la table des matières }}$}

Les organes de la vie intérieure, liés par une sympathie étroite et nécessaire avec le centre de réaction cérébrale, doivent correspondre aussi par cet intermédiaire avec un siège supérieur, celui d'où partent les déterminations d'une force constante qui n'a pour caractéristique de ses actes propres que le sentiment de puissance ou de liberté, qui en accompagne toujours plus ou moins l'exécution.

De là le mélange constant des phénomènes de l'intelligence à ceux de l'affectibilité simple dans cet ordre mixte que le langage exprime sous le terme propre de passion.

Certaines images, spontanément conçues dans leur siège, vont exciter des affections, imprimer un nouveau ton aux organes internes et changer souvent l'ordre des fonctions vitales.

Réciproquement, ces affections, résultats immédiats du jeu de la vie, déterminent, forcent souvent, dans le siège le plus rapproché de celui de l'intelligence, la production correspondante d'images appropriées à tel état organique. Lorsque ces dernières sont liées à des signes institués, la volonté y aura quelque prise, pourra prendre part dans la passion même, y amener des 
produits d'un autre ordre, en changer quelquefois le cours. Cette sorte d'influence pourtant, n'étant ici que médiate et éloignée, est toujours trop incertaine, variable et subordonnée toujours jusqu'à un certain point aux dispositions propres des organes, placés hors de la sphère de la volonté. En attachant, par exemple, l'attention à une image dont nous avons le signe disponible, nous pouvons produire quelque émotion passagère, qui se convertit souvent en passion réelle, ou en un sentiment durable, par la répétition du même acte. La même affection peut naître aussi immédiatement, et forcer la production, la réoccupation de la même image opiniâtrement persistante, malgré tous les efforts de la volonté pour la distraire.

Tels sont les liens et les rapports de subordination de deux sortes de vie. C'est ainsi que la puissance de vouloir est souvent opprimée, ou n'existe plus comme puissance, mais c'est ainsi qu'elle peut quelquefois opposer aussi avec succès les souvenirs aux impressions, aux images et, par certains signes de réclame, apaiser les orages comme concourir à les exciter.

C'est dans la prédominance ou l'initiative de l'un ou l'autre ordre d'éléments ou de phénomènes, dont se compose plus ou moins toute passion, dans un certain développement du moral que me paraît être la seule ligne qui sépare ce même moral proprement dit du physique, dont on restreint ou l'on étend trop, tour à tour, les limites, soit qu'on place le moral où il n'est pas, soit quand on ne le voit pas où il est.

Un sentiment quelconque doit être caractérisé comme moral, lorsqu'il naît à la suite d'un acte ou d'un travail intellectuel, et que la volonté, en possession de ses moyens d'influence, peut y prendre médiatement quelque part, ainsi que nous venons de le dire. Une passion, qui porte certains signes apparents d'intelligence, me paraîtrait au contraire pouvoir être caractérisée comme appartenant au physique, lorsque les actes qui y entrent sont des résultats des dispositions ou de jeu de la machine, et que la puissance du vouloir n'a aucun moyen, actuel disponible, pour la modérer ou la suspendre.

La partie affective et purement organique des passions a été quelquefois rapportée tout entière à l'intelligence, comme l'effet à la cause ; on en trouve entre autres un exemple dans ces paroles remarquables de Descartes dans une lettre à Élisabeth sur ce sujet: tota autem nostra voluptas posita est in perfectionis alicujus nostrâ conscientiâ. L'école de Leibnitz se trouve placée à peu près dans le même point de vue au sujet des modes du plaisir, et de la douleur en général, considérés comme des résultats immédiats de l'activité de l'âme pensante ; quelquefois au contraire, tout dans la passion se trouve réduit à la partie organique, l'intellectuelle y étant méconnue ou identifiée avec le résultat, à l'affection immédiate. On en trouve un exemple dans cette assertion trop générale d'un physiologiste célèbre (Bichat) : les passions ont tout leur siège dans la vie organique. C'est entre de tels extrêmes qu'il faudrait se placer, 
je crois, si l'on voulait chercher le fondement réel d'une division ou d'une sorte de classification de ces phénomènes mixtes, division qui ne saurait ressortir exclusivement de considérations absolues ou relatives à un seul élément du composé.

Si c'était ici le lieu d'approfondir cette matière et d'appliquer la méthode de décomposition dont nous avons fait usage auparavant, nous serions probablement conduits à diviser toutes les passions humaines en trois classes respectivement fondées sur un certain ordre de prédominance ou d'équilibre, que peuvent observer entre elles les deux forces qui y concourent, ou les instruments qu'elles mettent en jeu; d'où résultent des produits, dont on ne peut méconnaître la différence dans certains signes pris au moral comme au physique.

La première classe, par exemple, comprendrait les passions simples et naturelles que nous avons déjà distinguées sous le titre d'affections, ou de sensations générales, lorsqu'elles sont unies à une forme personnelle. Ces passions primitives diffèrent à peine de l'instinct nutritif et conservateur, qui est avant la connaissance et continue à donner l'impulsion, lors même qu'il s'ajoute avec des facultés d'un autre ordre ${ }^{1}$. Ici l'imagination reçoit bien toute la loi des organes de la vie intérieure, et le désir résultant (premier composé de cet ordre sensible), identifier avec le besoin plus immédiat comme affection, ne s'en distingue que par le jugement d'expérience qui lui donne un objet au dehors ou l'attache à une image dans le souvenir.

La deuxième classe comprendrait toutes les passions artificielles et bien composées qui ne peuvent naître que dans un certain degré de développement moral ou un ordre éventuel du progrès des sociétés : telles sont l'avarice, l'ambition, l'amour de la gloire ou des distinctions en divers genres, etc., qui, hors des besoins primitifs, arrachent l'homme à une certaine torpeur naturelle, mettent en jeu son activité, le poussent dans une certaine carrière et puis l'y fixent, l'y rattachent par les chaînes de l'habitude ou l'empire d'un autre ordre de besoins. Ici l'imagination et le travail de l'intelligence prennent l'initiative et conservent toujours l'influence la plus apparente. Cependant, l'habitude ne fait la passion proprement dite, qu'en plantant pour ainsi dire ses racines dans le fond des organes de la vie intérieure, ou modifiant persévéramment, ces centres d'affectibilité, de telle manière que l'ordre renaissant de leur action sympathique ramène constamment les mêmes images, commande l'attention et force la répétition de ces mêmes actes dans l'intelligence, qui deviennent effets

\footnotetext{
Sic. Il nous semble qu'il faut lire : "Lors même qu'il s'y ajoute des facultés d'un autre ordre. » Le style de cette dernière partie est particulièrement pénible, et paraît justifier la crainte exprimée par Maine de Biran, dans son avant-propos, que son manuscrit, en divers endroits, fût peu lisible. (P. T.)
} 
nécessaires après avoir été cause déterminante ${ }^{1}$, à ce terme d'habitude qui met une sorte de fatum dans la passion même ${ }^{2}$. Tout semble bien rentrer, en effet, dans l'ordre physique, mais le fondement de la division particulière que nous aurions établie subsisterait toujours, eu égard au premier mobile de la passion, à la nature de son objet, à la part qu'y prend la conscience et celle que l'activité d'un vouloir bien déterminé pourrait y recouvrer encore.

La troisième classe renfermerait les passions ou affections que j'appellerai plus particulièrement mixtes ou sociales, eu égard à une sorte d'instinct social affectif, inhérent peut-être au naturel de l'espèce humaine, instinct qui est la base de toute moralité, qui s'étend et se concilie toujours avec les progrès de la réflexion, se complique et se dénature par les effets d'une imagination trop exaltée dans son principe, se perfectionne enfin par une bonne éducation première, comme il se dégrade et paraît s'effacer par les exemples et l'imitation d'une société corrompue. C'est cet instinct ou sens moral non perverti qui attache l'homme à l'homme et lui rend son bonheur sacré. Là est la source pure de toutes les passions bienveillantes, de tous les besoins d'un être intelligent et sensible. Là est cette sanction complète de la loi de nature, qui n'a pas séparé le devoir du plaisir ou de la récompense, ni l'infraction de la peine ou du châtiment ${ }^{3}$.

Les sentiments moraux dont nous parlons se distinguent bien de toutes les autres passions; quoiqu'ils puissent être immédiats et spontanés dans leur principe, ils se fortifient et se doublent par la réflexion en concourant même heureusement à son exercice, tandis que les affections, dont la source est uniquement et primitivement organique, absorbent plus ou moins complètement cette faculté ou disparaissent de la conscience dans son exercice.

Tel est le caractère attaché à tous les sentiments de cet ordre qui naissent d'un emploi utile et régulier de toutes nos facultés actives. C'est sur de tels caractères ou sur les conditions organiques mêmes auxquelles on peut les rattacher comme signes, que me semblerait devoir se fonder la classification et

C'est sur cela qu'est fondée la sage maxime qui renferme toute la morale préservative : principiis obsta.

Sic.

Je crois qu'on pourrait trouver là une preuve confIrmative de la réalité de cet instinct moral admis par quelques philosophes et rejeté par d'autres. En effet, le méchant est toujours essentiellement malheureux, indépendamment même de toute réflexion ou prévoyance sur le résultat de ses actions perverses; le malheur est pour lui une affection immédiate, c'est comme un animal dont l'instinct primitif serait contrarié ou perverti. Par la même cause, l'être bon et bienveillant est immédiatement heureux, hors de la réflexion même, qui double ses jouissances intérieures; il sent directement et sans aucun retour qu'il est bien ordonné suivant ses véritables rapports de nature.

En outre, l'habitude flétrit bientôt infailliblement toutes les jouissances factices ; mais les plaisirs que donne la bienveillance sont inaltérables, et ne changent pas plus au moral, que le goût pour les aliments appropriés à l'instinct ne change au physique; c'est pour nous comme le goût du pain et de l'eau. 
l'analyse théorique de ces passions ou sentiments, dont la morale calcule les effets et donne les règles de direction pratique ${ }^{1}$.

Il ne faut pas confondre avec les passions ces émotions passagères qui peuvent naître de nos actes mêmes intellectuels, ou qui, provenant des saillies accidentelles de l'organisation, où elles n'ont pas pris racine, déterminent souvent la production de certaines images, qu'elles teignent de leur couleur. C'est encore sur une telle distinction que l'on pourrait fonder, avec plus ou moins d'exactitude, une sorte de classification de ces modes distingués par LOCKE, sous le titre propre de modes du plaisir et de la douleur, et compris si vaguement sous le titre de volonté. Il importerait, ce me semble, de distinguer les cas où ces émotions accompagnées de certains jugements ou rapports vraiment sentis d'une manière sympathique, comme dans la joie, la tristesse, l'espérance, la crainte, etc., naissent réellement des actes intellectuels ou jugements, des cas où les affections inhérentes à un certain état organique déterminent au contraire et forcent le sentiment des rapports des êtres et des choses avec notre susceptibilité sensitive. On pourrait reconnaître peut-être par la mobilité de ces rapports sentis, comparés à l'invariabilité de ceux qui sont réellement perçus la diversité des sources auxquelles ces deux sortes d'opérations se rattachent.

Mais je n'ai déjà que trop insisté sur un sujet qui, dans son rapport avec la science des facultés intellectuelles, se trouve déjà assez approfondi ailleurs, et qui ne pourrait entrer dans ce mémoire, en lui donnant l'extension qu'exigerait le point de vue moral auquel il est spécialement afférent.

La faculté particulière que nous avons nommée imagination sensitive, considérée sous le rapport des affections que son exercice fait naître, ou dont il reçoit les lois, se trouvant placée pour ainsi dire entre les deux sortes de vie dont elle forme le lien, emprunte également de l'une et l'autre source. Ainsi, une image, présente à la pensée, porte toujours avec elle le caractère et comme le cachet de sa force productive ; intellectualisée dans l'acte qui la rattache à un signe volontaire, elle se sensibilise entièrement dans la cause organique qui la suscite et la provoque. Dans ce dernier cas on reconnaît tous les caractères dominants d'une faculté sensitive ; on la reconnaît à la légèreté et à la bizarrerie de ses produits, comme à leur opiniâtre persistance, et quelquefois à leur élévation surnaturelle ; on la reconnaît à ses transports tumultueux, à la rapide succession des espérances et des craintes, à l'intérêt toujours vif et dominant qui s'attache à ses tableaux et à toutes ses créations.

L'influence réciproque de nos facultés affectives sur nos idées ou opérations intellectuelles, et vice versa serait le sujet intéressant d'un ouvrage que me semble attendre encore la morale théorique ; un tel sujet ne doit pas être traité en passant, et mérite bien d'être approfondi. 


\section{Chapitre III}

\section{Ordre actif intellectuel}

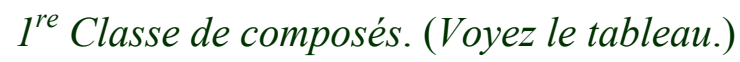

\section{De l'imagination intellectuelle spontanée et réglée par l'emploi des signes institués.}

\section{$\underline{\text { Retour à la table des matières }}$}

Les philosophes ont assez généralement convenu de donner le nom d'imagination à une faculté mixte, qui s'applique à faire de nouvelles compositions d'idées, ou à rassembler dans une sorte d'objet fantastique diverses qualités éparses dans les êtres réels. Ils caractérisent cette imagination sous le nom d'active, en la distinguant ainsi de cette faculté toute passive, dont l'exercice direct et spontané fait plus qu'étendre pour nous les limites de la sensation même, puisqu'elle la fait revivre. Ainsi considérée, l'imagination serait moins une faculté particulière et sui generis, qu'un concours de presque toutes celles que l'on a rangées sous les titres séparés d'entendement et de volonté.

Le seul caractère distinctif serait ici l'emploi et la direction de ces facultés diverses vers un but particulier, qui consiste à émouvoir la sensibilité par des peintures séduisantes ou terribles, l'exciter par des contrastes, l'étonner par de grands ensembles. C'est toujours sur quelque effet excitatif de cette nature, que se fonde le titre de cette faculté supérieure d'imaginer, et le nom qu'on lui conserve indique de lui-même la source d'où elle tire les matériaux de ses créations comme les rapports intimes, qui la lient aux dispositions naturelles ou acquises de l'affectibilité directe. 
L'organe central, monté au ton des impressions que les sens externes lui ont transmises d'une part, et surtout de celles qu'il reçoit sympathiquement des centres partiels de la sensibilité intérieure, n'a besoin d'aucun travail commandé, d'aucune impulsion étrangère, ni d'aucune force directrice pour créer de véritables archétypes sans modèle et sans règle, et faire des compositions d'images, des peintures de fantaisie de toute espèce. Les songes, les divers degrés d'aliénation mentale, et sans aller si loin, ce que nous éprouvons à chaque instant, en laissant marcher notre cerveau sans aucune contrainte, nous fournissent assez d'exemples de ses créations spontanées

Mais ce sont ordinairement des produits éphémères que le même instant voit naître et mourir ; une disposition organique particulière les enfante; elle passe, et les fantômes avec elle.

SYSTÈME INTUITIF INTELLECTUEL. - Lorsque l'individu est en possession des signes institués, et que toutes les facultés, développées par leur usage, ont acquis cette extension, cette régularité d'exercice, qui constituent ce que nous appelons raison humaine, l'organe propre de l'imagination, ayant été travaillé de toutes les manières se trouve, pour ainsi dire, muni d'une quantité prodigieuse de matériaux ; il les combine encore le plus souvent sans s'asservir à aucune loi, il crée des modèles et ne se règle sur aucun; mais ses produits spontanés, en se rattachant, à des signes volontaires, prennent un caractère plus intellectuel, plus persistant, et peuvent se prêter ainsi aux nouvelles élaborations des facultés actives.

Quelque extension que puisse acquérir cette faculté mixte de production et de combinaison des images sensibles, à quelque éloignement qu'elle nous paraisse de sa source, lorsqu'elle s'élève et plane sur toutes les merveilles d'un monde idéal, elle porte, toujours l'empreinte de sa première origine. C'est la partie la plus sensible de nous-mêmes, celle qui constitue notre caractère et nos passions, qui inspire souvent et contribue à la création spontanée des prodiges de l'art ; elle s'empreint dans les tableaux du génie quels que soient les signes matériels qui nous les retracent. (couleurs, sons ou paroles); nous la sentons, nous sympathisons avec elle; elle anime encore le témoin de la création comme elle anima le créateur. Cette forme ineffable d'inspiration, source des pouvoirs les plus étonnants de l'homme, est elle-même hors des limites de sa puissance ; bien plus, elle cesse d'exister et perd tout l'ascendant qu'elle a pour nous émouvoir, dès que la volonté tend à lui donner des lois, ou cherche à reproduire ou à imiter son charme suprême.

On pourrait dire de cette faculté de créer en représentant, qu'elle se définit elle-même uniquement par son exercice. Opposée à la tranquille et froide réflexion, jamais elles ne coexistent ensemble ; celui qui obéit actuellement aux inspirations du génie est sous le charme; il ne se rend pas compte de ce 
qu'il fait ou de ce qu'il éprouve, il sent ou voit directement et n'aperçoit pas, et lorsqu'il aperçoit, il ne sent plus, ou n'imagine plus ; mais si le génie n'est pas lui-même dans le secret de sa puissance, qui pourra chercher à le surprendre ?

Le génie des arts est en quelque sorte comme le despotisme : il est doué de la force exécutive; cela lui suffit. Sa force législative est dans l'exécution même. Il est une sorte d'exercice de cette faculté d'intuition qui, toujours plus ou moins indépendante dans son excursion et ses saillies, se trouve plus près du champ de la réflexion par le caractère de ses produits, l'espèce de signes qu'elle y rattache, les méthodes mêmes qu'elle se fait et à qui elle peut encore demeurer fidèle jusque dans son essor le plus hardi. Placé au sein de ces masses qui sont pour lui comme des éléments du monde idéal, le génie des sciences appuyé sur un petit nombre de signes très complexes et très généraux qui doublent son énergie en la concentrant, saisit à la fois les lois et les rapports les plus étendus de ces masses, en même temps qu'il pénètre dans la constitution intime de chacune : il revit les abstraits dans les concrets et les concrets dans les abstraits. Toujours guidé dans sa marche ou son vol par certaines analogies, souvent le premier aperçu est une inspiration secrète qui lui semble descendre du ciel et dont lui-même ne se rend pas compte; quelquefois elles lui sont suggérées par le rapport des signes mêmes; c'est là que sa sagacité les aperçoit ou les devine, et c'est de là qu'il part pour trouver une analogie de la nature, quelqu'une de ces grandes lois qui régissent le système des êtres.

Kepler, méditant sur certaines propriétés des solides géométriques, est averti, comme par une sorte d'inspiration vraiment intuitive, qu'il doit en exister un parallèle entre les temps périodiques des planètes et les distances au centre de leurs révolutions ; il suit cette idée, tâtonne, essaie différents rapports de nombres et de leur puissance, trouve le véritable que l'observation transforme en lois de la nature.

Newton voit tomber le fruit d'un arbre ; son génie s'élève par une intuition spontanée jusqu'à la sphère de la lune, pressent l'identité de force qui fait tomber une pomme, et retient les corps célestes dans leur orbite. D'immenses calculs vérifient encore cette opération.

Quelles analogies apparentes pouvaient conduire Franklin à soupçonner l'identité de la foudre et de l'étincelle électrique ? Plusieurs, nous dit-il luimême : la lumière, la couleur de cette lumière, sa direction en zigzags, la rapidité du mouvement, la facilité à se laisser conduire par les métaux, etc. Sans doute, tous ces moyens confirment le premier aperçu du génie, ils comblent tout l'intervalle qui sépare la nuée étincelante et la majesté des orages, d'un mince appareil électrique ; mais il fallait que l'imagination franchît la première cette distance, et qui est-ce qui la dirigea dans cette hardie excursion? 
Cette indéfinissable faculté créatrice n'a point pour fonction de désemboîter, pour ainsi dire, des idées ou des termes renfermés les uns dans les autres, ni de suivre régulièrement le fil d'une prétendue identité logique qui les unit.

L'imagination seule, emportée souvent par une saillie heureuse, fixe des points éloignés et les mesure déjà de son rayon ; quelquefois elle se crée ellemême les moyens intermédiaires qui doivent combler l'intervalle qui les sépare. D'autres fois, elle indique la possibilité seule du rapprochement et en abandonne les moyens à nos méthodes techniques. Peut-on ramener exclusivement ces cas divers à l'influence des méthodes? Dira-t-on que l'invention, dans les beaux-arts comme dans les sciences, est toujours également l'esclave des formes de l'analyse?

Corneille et Newton sont deux génies inventeurs dans des genres opposés. On peut les comparer sans doute par le principe même de l'invention, par cette spontanéité du génie qui devine et sent, comme par un premier instinct, le beau et le vrai, si identiques dans leurs sources, s'élance avec enthousiasme vers cette image et puise, dans sa jouissance anticipée, les forces nécessaires pour en atteindre la réalité. Sans doute, le feu sacré qui inspirait au poète ces traits du sublime, brûlait aussi dans l'âme du géomètre qui entrevit pour la première fois l'admirable chaîne de rapports qui lient entre elles les sphères roulantes. Ils l'éprouvaient aussi dans toute sa force, cet enthousiasme, source de toutes les découvertes, ce Kepler si transporté de joie après avoir trouvé une loi cherchée avec tant d'ardeur, et avant lui ce Pythagore, cet Archimède dont l'histoire nous peint les transports, dans la jouissance et la contemplation de l'immortelle vérité.

Le principe qui invente est le même sans doute, quels que soient les objets ou les produits de l'activité créatrice. Ce principe indéfinissable réside dans une certaine chaleur de l'âme, peut-être dans une correspondance libre et facile, entre le centre organique où s'allume le sentiment et le siège où brille et règne l'intelligence. Mais dans l'exercice de facultés diverses, les deux foyers n'agissent pas également, ni dans le même ordre d'influence.

Pour le poète, c'est du cœur que tout part ; c'est à lui que tout revient, c'est par son imagination que l'esprit continue à se diriger. Pour le philosophe, le sentiment qui précède ou qui suit l'intuition, n'entre que comme encouragement ou récompense ; il peut embellir l'entrée ou couronner la fin d'une carrière dans laquelle l'intelligence demeure livrée à ses propres efforts.

Rien ne se ressemble moins, au surplus, que les moyens d'exécution et la marche de l'esprit inventif dans les deux cas comparés : le philosophe et le poète emploient également des signes, des formules ; mais l'image sensible est 
avant la forme dont elle se revêt en lui donnant son empreinte, tandis que le concept intellectuel est indivisible de son signe et n'existe souvent que par lui. Le philosophe sent que toute sa force est dans sa méthode et s'y appuie avec confiance. L'imagination du poète se soumet à regret aux formes du langage, lutte contre elles, les dompte quelquefois et même en leur cédant fait preuve d'indépendance; enfin, l'une dépouille les signes pour simplifier, l'autre les compose pour émouvoir ; pour l'une les signes sont des leviers, pour l'autre ce sont des talismans.

S'il est prouvé, comme je le pense, que les méthodes mécaniques n'entrent point dans nos raisonnements sur les faits, comme dans ceux qui ont uniquement pour objet la comparaison de nos idées ou de nos termes abstraits, à combien plus forte raison ces méthodes doivent-elles être limitées dans leur influence, lorsqu'il s'agit d'une faculté qui est toute en tableaux et en sentiments, d'une puissance de création qui, dépendante dans son exercice du concours d'une multitude de circonstances, parmi lesquelles les dispositions sensitives doivent entrer en première ligne, résiste au frein des méthodes et à l'emploi de tous nos moyens artificiels.

Parmi les caractères divers que prend cette faculté multiforme que l'on nomme imagination ou intuition, le point de vue le plus fixe, sous lequel nous puissions la saisir, est celui de la correspondance constante qui s'établit par elle entre nos facultés affectives et intellectuelles ${ }^{1}$. C'est, pour ainsi dire dans le

C'est pour ainsi dire dans le point de contact des deux vies, sensitive et intellectuelle, que se trouvent placées toutes nos plus douces jouissances; c'est là ce qui rattache au sentiment mixte de l'amour moral (quoique en ait dit Buffon), nos voluptés les plus ineffables. On peut remarquer aussi qu'il n'y a pas de moment plus heureux que celui où l'un satisfait un besoin physique avec l'esprit ou le cœur prévenus d'une idée ou d'un sentiment agréable... comme si l'on jouit pendant les repas de la conversation intéressante d'un ami, etc. Ce rapport intime qui lie nos facultés affectives à certains exercices de nos facultés intellectuelles, se montre bien dans la manière dont nous sentons réellement les rapports intimes de nos idées avec certaines dispositions de notre affectibilité intérieure.

Les créations les plus constantes même de notre pensée, contemplées tour à tour en dedans avec le sentiment de force ou de la faiblesse, de sérénité ou de trouble, nous affectent d'une manière bien différente ; elles semblent se teindre, comme les objets euxmêmes, des couleurs propres de notre affectibilité.

Remarquons enfin que les idées les plus persistantes et qui marquent le plus dans notre durée, sont celles qui se lient à un sentiment et où les deux vies prennent une part égale, car pour peu que l'affectibilité domine, il n'y a plus de souvenir. Voilà pourquoi les enfants, dont la vie est presque sensitive, sont absolument sans mémoire, et pourquoi les vieillards aussi qui sentent si peu ont l'imagination absolument éteinte et la mémoire presque nulle. Ils conservent l'identité personnelle jusqu'à l'entière caducité, mais la réminiscence modale ne s'exerce pas, ce qui s'explique parfaitement dans nos principes. On comprend bien aussi pourquoi les mêmes vieillards doivent bien mieux se souvenir de ce qui leur est arrivé dans une époque reculée que de ce qu'ils ont fait dans les temps les plus rapprochés ; c'est que les anciennes déterminations avaient pris un double point d'appui dans les deux vies, et qu'à mesure qu'on avance, l'un de ces points d'appui s'affaiblit jusqu'à ce qu'il finisse par manquer entièrement. Mais ces souvenirs lointains des vieillards ne sont que des 
point de contact de ces deux ordres de facultés, que l'imagination créatrice se place et exécute toutes les compositions ; tantôt elle forme avec des matériaux empruntés de l'entendement ces idées archétypes qui la dirigent dans de nouveaux procédés, lui découvrent un plus vaste horizon; on reconnaît son influence dans ces aperçus hardis et rapides du génie, qui prend spontanément son vol vers un ordre de vérités placées bien au-delà de la sphère de son siècle, jette les fondements d'une science nouvelle, et prépare tous les progrès futurs des générations ${ }^{1}$.

Souvent l'imagination emprunte d'une nature sensible, mais plus parfaite que celle qui frappe nos yeux, les éléments de ces compositions prototypes du beau idéal dans tous les genres. Ses tableaux vivants excitent toute notre sensibilité ; un charme particulier, une sympathie profonde s'y rattachent, et l'on peut connaître, à l'émotion qu'ils excitent, à la manière dont on les sent, plutôt qu'on ne les juge, la source d'où ils partent et la faculté dominante qui les inspira.

Dans la faible esquisse de ces traits qui caractérisent une sorte d'intuition ou imagination intellectuelle, nous pouvons reconnaître une faculté spontanée dont l'exercice actif est tout en résultat, et non point en principe d'aperception ou de conscience. Tel est le caractère de cette faculté qui s'exerce, pour ainsi dire, en dehors de l'être pensant. C'est aussi sous ce rapport extérieur, ou en la jugeant par ses résultats, qu'on peut lui donner la qualification d'active par

réminiscences d'idées sensibles auxquelles certaines liaisons ou rapports de circonstances les conduisent ; car il est certain que la dégradation de l'âge exclut la véritable mémoire des idées acquises et des opérations intellectuelles exécutées dans toute la vigueur des facultés. C'est ainsi que d'Alembert dans les dernières années de sa vie avait perdu la mémoire des problèmes qu'il avait résolus dans sa jeunesse.

Ces divers exemples et une multitude d'autres que je pourrais citer, me paraissent bien propres à confirmer la distinction établie entre les deux ordres des facultés. C'est en observant l'influence qu'elles exercent les unes sur les autres dans une multitude de cas, puis en les comparant dans leur isolation, qu'on apprend mieux à signaler leurs caractères, et à ne pas les confondre sous le même titre. Assurément, quelque envie que l'on ait de simplifier, il est impossible que le terme unique faculté de souvenir puisse s'appliquer nettement à la reproduction d'une image, ou accompagnée de réminiscence, ou sans réminiscence, et encore au rapport des actes intellectuels avec l'identité personnelle qui s'y joint. Si l'on renfermait toutes ces idées réellement très distinctes sous un titre d'une faculté complexe, comment pourrait-on trouver le caractère d'un élément de l'intelligence ?

Lorsqu'une découverte est mûre, pour ainsi dire, plusieurs hommes y parviennent ordinairement, chacun de leur côté, par les seules forces de l'analyse. Divers mathématiciens trouvèrent en même temps les lois du mouvement et du choc des corps. Newton et Leibnitz, inventèrent chacun de leur côté le calcul différentiel; mais comme le genre de ce calcul se trouvait renfermé dans des méthodes connues, telles que la méthode des tangentes de Bacon, celle des indivisibles de Cavalieri, etc., Il est possible que, tôt ou tard, des mathématiciens, doués de moins de génie, eussent fait la même découverte. Mais il n'y avait peut-être qu'un seul homme tel que Kepler, dont l'imagination était la faculté dominante, pour trouver cette grande loi qui devait donner, dans la suite, les clefs du système du monde. 
laquelle on la distingue souvent. N'est-ce pas de la même manière qu'on reconnaît une activité des passions ? Ainsi, c'est lorsqu'un être est le plus complètement passif pour lui-même ou dans sa propre aperception, qu'il est le plus actif aux yeux du spectateur, et ici l'opposition dans les termes décèle bien l'opposition qui existe entre les deux points de vue, dont l'une se fonde sur une intuition toute externe, tandis que l'autre se concentre dans la réflexion la plus intime : c'est là qu'est le secret de toutes les divergences des systèmes métaphysiques et la source de tant d'obscurités apparentes. Si nous avions une langue expresse appropriée à la réflexion, nul doute qu'il y aurait une évidence métaphysique comme une évidence mathématique.

En effet, les deux ordres de conceptions sont tirés, sans mélange, de la source même de l'évidence, s'appuyant chacun sur l'un des éléments du même fait primitif, celui de conscience de l'existence aperçue (voyez le chapitre du toucher). Mais toutes les habitudes premières de notre sensibilité, et par suite celles du langage qui s'y conforment, nous rapportant hors de nous, objectivent d'abord toutes nos conceptions; on voit bien pourquoi la culture de la science de nous-mêmes ou de nos propres facultés doit être toujours rare, difficile, rebutante et sujette à mille sources d'illusions pour ceux-mêmes qui sont le mieux et le plus sincèrement disposés à la cultiver.

La faculté créative spontanée dont nous venons de parler est toujours représentée dans le langage sous l'emblème de la vue, intueri, voir ; on dit l'œil perçant du génie, et c'est bien, en effet, dans les opérations infiniment promptes, faciles et comme spontanées de ce sens, que les découvertes du génie trouvent leur représentation la plus fidèle; et peut-être cette dernière observation concourt-elle à justifier la marche que nous avons suivie en ralliant, non toutes les mêmes facultés nominales à la sensation en général, mais un ordre de facultés particulières à l'exercice de chaque sens.

L'un des caractères les plus propres à distinguer l'intuition de l'aperception, c'est que, la réminiscence, qui entre exclusivement dans les produits relatifs de l'une, ne s'attache point aux produits spontanés de l'autre, qu'une idée, un rapport nous frappe vivement comme d'un trait de lumière, ainsi qu'il arrive souvent dans certaines dispositions heureuses où les idées viennent nous chercher, sans que nous nous en mêlions (par exemple en voyageant, en mangeant, etc.) ; quelle que soit leur vivacité et leur netteté, si nous ne les rattachons pas de suite à quelque signe disponible, si elles ne sont pas soumises à une sorte d'incubation de la pensée, elles nous échappent entièrement et nous sommes tout étonnés bientôt après, de les chercher en vain : si elles se représentent, il doit arriver souvent aussi qu'elles nous paraissent tout à fait nouvelles; et n'est-ce pas la raison par laquelle l'homme de génie est souvent incapable de bien tracer la carte de sa route et de donner la description ou l'analyse de ses procédés ? Eh ! ne sommes-nous pas tous dans le même cas par rapport à ces opérations intellectuelles que nous exerçons dans une sphère plus 
étroite ? N'est-ce pas pour cela que la science de nos facultés est si peu avancée et que le problème sur lequel j'ai bégayé si longtemps n'aura peut-être encore qu'une solution imparfaite? 


\title{
Chapitre IV
}

\section{Ordre actif intellectuel}

\author{
$2^{e}$ classe de composés (voyez le tableau).
}

\section{I - De la mémoire ou du rappel des idées par des signes disponibles.}

Memoria non est imaginum custos, sed facultas quœ ex rebus mente conceptis propositum denuo promere potest. (porphyre.)

\section{Retour à la table des matières}

Cette proposition énoncée par un philosophe qui écrivait dans un temps où la science des facultés humaines, placée et rattachée au ciel, se trouvait hors de l'humanité même, cette proposition dis-je, très remarquable, me paraît peindre en un seul trait le caractère complet de la faculté intellectuelle que nous appelons mémoire, lorsque sentant qu'il entre dans le rappel de nos actes et de nos idées quelque chose de plus que dans une simple vertu représentative des choses extérieures, nous voulons nettement établir la différence en nousmêmes et la consacrer sous un signe déterminé dans notre intime réflexion.

Il ne me reste guère qu'à commenter en peu de mots le passage de cet ancien, et à ramener à des conditions et des exemples sensibles le principe qu'il établit en résultat avec tant de précision et de justesse.

Ce sont ici encore, les opérations du toucher qui nous servent de point de départ. Dans l'exercice complet de ce sens se trouvent renfermées plusieurs circonstances qu'il importe bien de ne pas confondre : détermination 
volontaire, suite de mouvements ou d'actes, au moyen desquels la passivité de l'organe sensitif est mise à portée de son objet, aperception des actes mêmes ; perception de leurs résultats ; comparaison des uns aux autres dans le concours de la réflexion et de l'attention ; premier jugement ou rapport simple de moi au non moi ; deuxième jugement ou rapport composé des parties de l'objet et de ses qualités tactiles; tous ces modes, dis-je, sont renfermés dans l'exercice complet du sens considéré en dehors de l'habitude, car dans ces habitudes mêmes devenues si intimes tout paraît bien se réduire à une sensation simple, qui est du fait de l'objet bien plus que du nôtre.

Écartons cet objet. La même détermination volontaire peut s'effectuer de nouveau hors du concours de toute force extérieure, les actes se reproduire et être aperçus dans la propre détermination de leur puissance qui n'a pas changé ; la force de résistance (non moi), n'y est plus, mais le souvenir, qui s'y rapporte et la fait reconnaître comme absente, ressort encore d'un contraste ou d'une comparaison analogue à celle qui a établi son existence, ce qui suppose le souvenir de la comparaison première. Les qualités perceptibles de l'objet peuvent se retracer dans l'image qui en tient lieu, et pendant que l'attention s'applique encore en résultat à saisir leurs rapports composés, les actes qui déterminent ces rapports peuvent se réfléchir dans un sens plus intime; la seule chose qui manque absolument, c'est la sensation.

Maintenant que l'image tangible, ou ce qui en tient lieu (par exemple dans le cerveau d'un aveugle) se retrace avec une promptitude extrême et par une seule détermination rapide et légère, aussitôt effacée que conçue, comme il arrive dans l'habitude ; l'individu se représentera en masse l'image du solide, mais sans conserver la conscience ou le souvenir des actes qui ont nécessairement contribué à lui donner la forme actuelle ; alors il imagine, il conçoit, mais il ne rappelle point.

Il peut se faire aussi que, dans cet exercice comme spontané de l'imagination, il se joigne quelque retour sur les actes qui en ont déterminé l'origine : l'individu connaît alors un résultat partiel de son ouvrage dans l'image qui se représente ; il s'assure que cet ouvrage a existé, qu'il y a eu une suite de mouvements exécutés, de rapports perçus et dont l'idée totale de l'objet, maintenant claire et évidente par elle-même, a résulté. Mais il n'exécute point les mêmes opérations, il n'en a pas besoin puisque le but se trouve atteint d'avance. Ce souvenir d'actes précédemment exécutés, n'en est point le rappel, il n'existe pas non plus d'image, ni même de réminiscence objective qui s'y rattache ; toutes ces circonstances ne peuvent être confondues sous le même terme. La double détermination correspondante aux actes mêmes primitivement exécutés, et à leurs résultats perceptibles, peut être telle au contraire que ceux-ci ne s'accomplissent de nouveau dans le sens représentatif intérieur, qu'en tant qu'ils sont encore précédés des mêmes opérations, à la suite desquelles la représentation peut se compléter parfaitement, ou demeurer 
rebelle plus ou moins à l'acte du rappel; ce qui dépend des dispositions propres à l'organe de l'imagination, sur qui la volonté n'a point de pouvoir direct; ici le rappel est complet; dans le premier cas la mémoire s'exerce dans toute son étendue ; il peut être aussi considéré comme complet dans le second cas pour ce qui est objet propre du rappel; mais l'idée se trouve réduite à la forme dans la mémoire, la matière y manque (comme dans l'hypothèse précédente c'était la forme propre et constitutive de la mémoire qui manquait).

La réminiscence active s'exerce nécessairement dans ces deux dernières hypothèses, car la réitération volontaire des actes emporte le souvenir de la première exécution (effet qui n'est pas réciproque).

En reprenant, pour ainsi dire, en sous-œuvre et avec quelques détails de plus, tout ce que nous avons reconnu antérieurement dans l'exercice du sens spécial du toucher actif, nous nous trouvons encore avoir assigné avec assez d'exactitude les caractères, les moyens et circonstances principales de cette fonction intellectuelle qui consiste à rappeler des opérations et des idées déjà complètes, associées aux signes qui les surcomposent dans le sens intérieur de notre activité. Ces signes disponibles ne s'attachent point aux modifications affectives, nous l'avons assez vu; ils ne les représentent point en aucune manière ; ils ne sont là, pour ainsi dire, que pour attester à l'individu qu'il a été modifié, ou qu'il peut l'être encore, par une force en dehors de sa volonté ; ils peuvent assurer une sorte de réminiscence modale, mais ne la transforment jamais en mémoire.

Ces mêmes signes ne se combinent point non plus intimement avec les images qui sont les résultats de nos actes inaperçus dans leur propre détermination, car ces résultats conservent toujours en grande partie leur caractère de spontanéité apparente, et comme elles peuvent se reproduire sans le signe artificiel volontaire, il peut bien être rappelé sans elles, dans certaines dispositions du sens.

Les signes institués ne servent véritablement la mémoire ou ne s'approprient à cette faculté intellectuelle et ne la créent en quelque sorte une seconde fois, qu'en s'associant, comme produits homogènes d'une même puissance, à ces actes primitifs qui tendent incessamment à se confondre avec les résultats sensibles ou imaginaires, en leur assurant autant que possible, dans ces derniers mêmes, une sorte d'existence ou de signification à part, en donnant les moyens toujours disponibles de les concevoir, de les exécuter encore, ou de les suppléer par une réminiscence infaillible, en motivant enfin d'autres combinaisons plus étendues entre les actes mêmes ainsi isolés et des opérations nouvelles sur des signes d'opérations, qui, résumées ou conservées encore dans d'autres termes, multiplient à l'infini les actes de l'intelligence qui s'y réfléchit elle-même, cumulent ces actes les uns sur les autres, sans qu'il soit possible d'assigner un terme à la progression de cette échelle ascendante. 
D'ici nous contemplons l'arbre de la science, portant sa tête jusqu'aux cieux et couvrant de ses rameaux une surface immense, dont les bornes nous échappent. Et pourtant toutes les parties de cet arbre si prodigieux sortent d'un germe où elles étaient tout à l'heure renfermées dans l'exercice simple du sens, emboîtées les unes dans les autres, et se dérobaient à la vue microscopique, comme elles sont maintenant hors du champ de la vue directe.

\section{II. - Divers caractères de la mémoire.}

\section{$\underline{\text { Retour à la table des matières }}$}

La mémoire intellectuelle a été transportée par presque tous nos modernes dans les résultats ou produits de représentation.

L'image étant tantôt considérée comme essentiellement accompagnée de réminiscence, ce qui la constitue souvenir ${ }^{1}$, tantôt entièrement séparée de cette réminiscence quoiqu'on lui conserve encore le même nom de souvenir ${ }^{2}$. Dans le premier cas on n'a point d'égard aux conditions qui fondent la réminiscence et peuvent lui servir d'appui hors de l'image même, on n'établit point, ce me semble, une distinction suffisante entre les divers modes d'exercice de cette faculté ; on ne remonte pas jusqu'à son véritable caractère constitutif. Dans le second cas, on établit le titre nominal de souvenir sur une condition hypothétique tout extérieure à l'être sentant; on l'affirme, pour ainsi dire, de dehors en dedans; c'est une simple qualification donnée à l'image ou à la sensation même reproduite ou prolongée ; on conserve seulement le signe en écartant la chose.

Dans ces deux points de vue il me paraît difficile de tracer d'une manière bien nette les limites qui séparent la faculté appelée mémoire, de celle que l'on continue d'appeler imagination, ou de voir là autre chose que des degrés ou quelques circonstances particulières de l'exercice d'une même propriété représentative ou reproductive des images, qui est comme la base à laquelle tout se rapporte. De là aussi les limites très étroites où l'on restreint cette faculté de rappel, qui embrasse tous les actes de l'intelligence et sans laquelle il n'y a point de véritable opération intellectuelle ${ }^{3}$.

\footnotetext{
Doctrine de Locke et de ses disciples.

Doctrine du Traité des sensations.

Lorsqu'on a parlé d'un siège particulier de la mémoire, c'est qu'on l'a confondue avec l'imagination sensitive, qui peut avoir réellement autant de sièges, qu'il y a de sensations spécifiquement différentes. Mais le rappel, étant un exercice de la puissance identique moi, n'a d'autres conditions, ni d'autres sièges qu'elle ; et là où cet exercice manque, il n'y a pas
} 
Les noms des objets directement perceptibles et capables d'en reproduire les idées directes, n'entrent que pour la moindre partie dans notre langage, et l'efficace réelle de ces noms eux-mêmes, lorsqu'ils sont régulièrement institués et associés, consiste moins encore dans la reproduction directe des images qui conservent toujours en partie leur caractère de spontanéité et d'indépendance, que dans le rappel des actes qui, ayant essentiellement concouru à la perception distincte d'un ensemble de circonstances et de propriétés, revivent encore par le signe dans la copie ou l'idée, la complètent ou la transforment en l'élaborant de nouveau.

Le signe unique associé à l'idée complexe d'une substance exprimée, je suppose, par le mot or ou fer, n'a point assurément pour fonction directe de représenter sous une image composée les idées des qualités ou propriétés si nombreuses que l'expérience y a successivement découvertes, dont la plupart consistent dans des rapports vraiment irreprésentables ; mais ces signes servent comme de points d'appuis fixes, auxquels s'attachent l'attention et la réflexion pour reconnaître, vérifier leurs travaux antérieurs, ou de points de départ, pour en recommencer de nouveaux.

Telle est donc la véritable fonction des signes du rappel volontaire dans leur association régulière à des produits homogènes de la même activité qui leur est inhérente. Ils assurent à ces derniers un second préservatif nécessaire contre la pente de l'habitude, qui les enveloppe dans les simples souvenirs des résultats les plus sensibles; ils conservent dans ces résultats mêmes les opérations de notre intelligence, comme le terme, mis en relief au dehors, résume en quelque sorte les opérations du toucher et les diverses opérations collatérales.

C'est ainsi que le signe oral écrit devient un monument fixe qui atteste à la pensée et son propre travail et l'objet, ou l'image à qui elle l'a consacré, et encore souvent les modes fugitifs, et irrévocables d'une autre existence.

C'est ainsi que la mémoire, suivant l'heureuse expression d'un philosophe, devient comme une digue que la pensée ose élever, au milieu du fleuve du temps ; j'ajouterai : et de ce cours rapide mais uniforme de l'habitude qui l'entraîne.

Nous pourrions dire d'une manière encore moins générale et plus rapprochée de notre sujet, que la mémoire remplit souvent à l'égard de la réflexion, dont elle est une dépendance essentielle, un office analogue à celui que la vue remplit à l'égard du toucher; en lui retraçant et lui faisant

réellement d'intelligence, quoique l'affectibilité organique ou cérébrale subsiste sous différents titres. 
reconnaître les résultats de ses actes, elle peut ou la dispenser d'un retour sur les opérations déjà exécutées, ou fournir l'occasion ou le moyen d'effectuer ce retour.

\section{III. - Comment on pourrait distinguer différentes espèces de mémoire.}

\section{$\underline{\text { Retour à la table des matières }}$}

Les signes volontaires associés aux divers modes, soit représentatifs, soit simplement affectifs, communiquent à tous, autant qu'il est possible, le caractère disponible et réfléchi qui leur est propre; il est très essentiel d'observer, relativement au dernier cas, que le terme d'associés ne s'attache point véritablement à l'impression affective, mais bien à l'idée de sa cause et de diverses, circonstances perceptibles qui accompagnent cette impression, dont elle peut être considérée elle-même comme une sorte de signe naturel ${ }^{1}$. C'est donc le jugement ou l'acte intellectuel, relatif à l'existence de cette cause ou à ses circonstances perceptibles, qui fondent le titre réel du signe dans la mémoire proprement dite.

Mais en tant que ce signe a déterminé par une association, directe ou indirecte, et en vertu d'habitudes actives ou passives, soit la reproduction des images, soit le réveil de quelque nuance d'une affection passée, l'acte de rappel, considéré ainsi dans ses résultats pourrait motiver la distinction de différents caractères mémoratifs.

Lorsque la représentation d'une image accompagne ou suit infailliblement le rappel du signe, en vertu d'associations régulières suffisamment répétées, la mémoire pourrait être dite représentative. Telle est sa fonction lorsqu'elle retrace les signes associés aux figures géométriques, etc.

Lorsqu'à ce même acte de rappel se joindra quelque degré d'une affection passée on pourra bien dire que la mémoire est sensitive ${ }^{2}$. Telle est celle qui

1 Thomas Reid a très bien observé que les noms par lesquels nous sommes censés exprimer nos propres sensations rapportées aux objets comme qualités, tels que les termes. chaud, froid, savoureux, etc., ne s'appliquent qu'à la cause de la modification et non point à cette dernière. Mais il me semble avoir beaucoup trop généralisé cette observation, en mettant sur la même ligne les sensations de goût, de l'odorat et du tact passif avec celles du toucher actif, de la vue, etc., et en supposant que toutes les sensations nous informent également de la cause extérieure. (Voyez l'appendice, du chapitre II.)

2 Voyez le Mémoire sur l'habitude. 
s'attache aux termes expressifs de quelques besoins ou états vagues de malaise, d'inquiétude, en général de toutes les sensations. (Voyez le tableau.) Mais dans ces deux espèces, ce n'est ni l'effet représentatif, ni le mode affectif qui caractérisent la mémoire ou constituent sa fonction, c'est la réminiscence qui accompagne l'acte volontaire du rappel, et se joint infailliblement à l'image (en formant ainsi avec elle une idée complète) ou aux traces obscures de l'affection primitive, et c'est ainsi que le signe atteste à l'individu qu'il a vu ou perçu, ou qu'il a été modifié à peu près, de telle manière actuellement irreprésentable. Voilà dans ces deux cas ce qui fait le caractère mémoratif inhérent à l'acte ou au terme volontaire et non point aux effets sensibles et au jeu des facultés passives, qui s'y joignent et s'en séparent tour à tour, suivant les dispositions capricieuses de l'organisation.

La mémoire considérée dans son caractère fondamental, ou dans le rappel des jugements et des actes intellectuels par les signes qui les expriment et les redisent, peut se diviser encore en intellectuelle ou réfléchie, en intellectuelle simple et en mécanique, ces trois titres lui appartiennent en propre : le premier en tant qu'elle conserve et peut retracer fidèlement l'ensemble des opérations ou jugements qui se trouvent résumés dans tel terme ou telle formule; le second, lorsque ces opérations ayant été exécutées antérieurement et les rapports ayant été évidemment perçus, le rappel des mêmes signes motive une réminiscence claire des résultats et de l'évidence première de ces rapports, ce qui dispense de vérifier de nouveau et de mettre encore la réflexion à l'épreuve (le jugement alors prend aussi le titre de simple réminiscence).

Le troisième, lorsque le rappel se borne à l'ordre des termes ou aux formules, les opérations qu'ils expriment n'ayant jamais été exécutées, ni les rapports perçus ou vérifiés, la réminiscence ne peut être dans ce cas que le souvenir même des anciennes répétitions, ou n'est que la continuation de ces jugements, que l'on pourrait très bien appeler mécaniques, ou d'habitudes ${ }^{1}$.

Mais la classe de surcomposés intellectuels de l'ordre, auquel nous avions principalement destiné cet article, se fonde surtout sur les conditions qui préparent ou effectuent le concours égal et l'équilibre des deux facultés de réflexion et d'attention, dont l'une a pour caractère de se concentrer sur les actes disponibles, tandis que l'autre s'attache à leurs résultats objectifs ou sensibles (et pour le dire en passant, on voit bien que tous les signes institués ne sont pas également propres à favoriser cet équilibre, et une langue écrite ou

La fonction importante que remplit la mémoire dans le jeu de toutes nos facultés me paraît nécessiter ces divisions ou distinctions spécifiques, qui n'intéressent pas seulement la théorie, mais encore et surtout la pratique de l'éducation intellectuelle. S'entend-on bien en effet, quand on parle d'exercer, de cultiver la mémoire ? Comment reconnaîtra-t-on le genre de culture approprié à celle qui mérite surtout notre attention, lorsqu'on confond sous le même titre un véritable mécanisme, destructif de toute capacité de penser ou de réfléchir? 
visuelle $^{1}$, appropriée à l'exercice de l'attention et de l'imagination, doit être bien moins favorable à la culture de la réflexion et de la mémoire, qui ne peuvent se passer d'une langue orale).

Dans l'association des signes institués à des modes actifs de nature homogène, ou à des idées déjà complètes, telles que les perceptions directes du toucher, de la vue et de l'ouïe, réunies dans un commun exercice, la volonté peut avoir en quelque sorte deux prises dans le composé intellectuel, mais l'une se trouve déjà comme annulée par l'habitude qui confond l'acte dans le résultat, et cette même influence s'étendant sur le composé comme sur le simple, sur l'ordre intellectuel comme sur le sensible, tend incessamment à masquer encore la fonction réfléchie du signe institué, et à faire prédominer dans le produit total la seule partie de l'idée qui peut s'objectiver au regard de l'attention.

Ainsi, dans une série plus ou moins longue d'actes volontaires qui se servent réciproquement de signes ou de moyens de rappel, depuis le premier jusqu'au dernier qui aboutit, je suppose, à un résultat sensible, l'effet constant de l'habitude est d'obscurcir indéfiniment la conscience de ces actes et des vouloirs successifs qui les effectuent, de les faire tous rentrer ainsi les uns dans les autres, de telle manière qu'après un certain nombre de répétitions, le premier moyen touche la fin qui parait être le terme, le but d'un vouloir unique, ou n'est plus en apparence que ce vouloir lui-même.

Cette loi générale de l'exercice de toutes nos facultés observées depuis l'acte qui accompagne et peut déterminer l'affection la plus simple ${ }^{2}$, jusqu'à l'opération intellectuelle la plus composée, nous explique bien comment la science proprement dite de nos facultés s'identifie complètement avec celle de leur emploi ou de leur objet, dès qu'on se place pour ainsi dire au centre même des habitudes, pour commencer l'analyse des sensations et des idées. Elle nous explique aussi parfaitement dans notre objet actuel, comment le troisième ordre de composés intellectuels, dont nous parlons, paraît ne plus faire qu'un avec le précédent, comment enfin la mémoire peut se confondre avec l'imagination, l'aperception réfléchie avec l'objective, etc.

Néanmoins autant il y a d'actes surajoutés, ou autant est étendue la série des intermédiaires qui séparent le premier mouvement disponible d'un mode qui ne l'est point par lui-même, autant aussi la prise de la volonté est infaillible, autant l'attention concentrée sur le résultat a d'énergie et de profondeur; le nombre de ces actes si rapidement successifs compensant en quelque sorte ce que

1 Le caractère de réflexion et d'activité, que la vue acquiert par l'association avec la voix et l'ouie dans cet art de la lecture, qui, tout simple qu'il nous paraît, est pourtant si admirable et si profond dans ses principes. Assurément la vue seule n'analyserait pas aussi parfaitement les caractères écrits, s'ils ne correspondaient pas à des articulations successives ou distinctes par elles-mêmes.

2 Voyez chapitre II de la $1^{\mathrm{re}}$ section : De l'Odoration active. 
l'habitude ôte de force et de persistance à chaque détermination en particulier, un tel exercice de l'attention redoublée équivaut presque à la réflexion et il suffit que la volonté n'y soit pas absolument effacée, pour qu'elle puisse raviver ses produits qui se soutiennent par leur nombre; se servir de l'un pour arracher les autres à la pente de l'habitude, et compléter de nouveau le caractère d'activité que cette dernière influence avait obscurci, tel est le grand avantage attaché à la pluralité des signes, qui conduisent à une pensée finale, en se traduisant les uns les autres. C'est là aussi que l'on reconnaît la véritable fonction de la mémoire, toujours prête à se confondre dans l'unité du signe avec l'imagination spontanée.

Tout ce que nous avons observé sur les moyens et conditions du rappel, comme sur les caractères de réflexion ou d'attention tantôt équilibrée, tantôt prédominante qui forme son exercice, me semble prouver que le centre d'action ou l'organe propre intellectuel auquel nous sommes conduits à rallier tous les ordres de composés dont il s'agit, ne déploie point réellement son activité sur lui-même ni sur la division cérébrale qui paraît être le siège propre de l'imagination. C'est bien ici le cas d'appliquer ce que Gassendi objectait à Descartes : Rien n'agit sur soi-même ${ }^{1}$.

«Considérant, disait GASSENDI (voyez les objections contre les Méditations de Descartes), pourquoi et comment il se peut faire que l'œil ne se voit point lui-même ni que l'entendement ne se conçoive point, il m'est venu à la pensée que rien n'agit sur soi-même ; car, en effet, la main, ou du moins l'extrémité de la main ne se frappe point elle-même, ni le pied ne se donne point un coup. Or, étant d'ailleurs nécessaire pour avoir la connaissance d'une chose, que cette chose agisse sur la faculté qui connaît, c'est-à-dire qu'elle envoie en elle-même son espèce, ou bien qu'elle l'informe ou la remplisse de son image ; c'est une chose tout à fait évidente, que la faculté même, n'étant point hors de soi, ne peut envoyer ni transmettre en soi soit, espèce, ni par conséquent former la notion de soi-même. Si l'œil qui ne voit pas en soi, se voit néanmoins dans un miroir, c'est qu'ils agissent l'un sur l'autre ; donnez-moi donc un miroir contre lequel l'esprit agisse de même façon, et alors il pourra se voir et se connaître, autrement je ne compte pas qu'il puisse avoir aucune idée de luimême. »

On peut voir (réponses aux secondes objections, t. II, des Méditations) comment Descartes se tire d'embarras en répondant que ce n'est point l'œil qui se voit, mais bien l'esprit, lequel seul conçoit et l'œil et le miroir et lui-même ; mais il me semble donner assez beau jeu à son adversaire, en entrant dans l'esprit de la comparaison, car il est très vrai, je crois, que si, nous étions réduits à des sens, tels que la vue, le sujet sentant ou représentant n'aurait point de moyens de se connaître ou de s'apercevoir directement dans son attribut propre d'action. Mais est-il donc vrai que rien n'agisse sur soi-même ? Le contraire ne peut-il pas être prouvé clairement par le témoignage du sens intime, sans recourir à des exemples étrangers, tel que celui de la toupie allégué par Descartes et qui ne prouve rien dans cette circonstance ? Est-ce que les différentes parties de notre corps, mues par la même volonté, ne font pas effort l'une contre l'autre ? Est-ce que cette puissance ne se sert pas d'un sens pour agir sur un autre ? Et pourquoi comparerait-on l'entendement à l'œil exclusivement, plutôt qu'à la main et surtout à la voix et à l'ouïe, etc. ? N'est-ce pas là un exemple de la possibilité d'agir sur soi-même, de s'informer, etc. ? Gassendi était physicien, et ne voulait introduire dans la métaphysique que des notions sensibles, des images; Descartes, au contraire, avait élevé dès son point de départ, un mur de séparation 
Pour qu'une force agisse, il lui faut un terme de déploiement. Or, ce terme auquel la volonté s'applique directement ne paraît pas plus pouvoir être ici l'organe cérébral même, que tout autre organe intérieur dénué des conditions de motilité volontaire. Les images se conçoivent, se produisent, se combinent, s'avivent d'elles-mêmes par l'exaltation spontanée ou anormale du cerveau (comme dans le délire, la manie, les rêves), sans qu'il s'y mêle alors aucun exercice de nos facultés actives. Dans l'exercice régulier de la pensée ou de modes potentiels que nous nommons, attention, réflexion, mémoire, jugement, etc., il y a bien certainement une action constante déployée hors du centre, et c'est sur ce déploiement disponible et excentrique que paraît se fonder la perception même des actes. Il n'a point lieu dans les visions extatiques, où tout semble se passer réellement dans l'intimité même de l'organe central ; mais quand le moi pense en demeurant compos sui, c'est toujours un sens externe activé par le vouloir qui lui fournit les signes de ses idées complètes, les moyens de ses opérations, en les lui réfléchissant en principe ou en résultat : c'est la vue, l'ouïe et la voix dans un homme qui pense en lisant, ou ce qu'il a lu ; l'organe oral surtout, et encore l'ouïe dans celui qui pense tout seul, et qui doit nécessairement entendre les idées dans toute la force du mot. Le sourdmuet doit penser en gesticulant en lui-même, ou en se représentant des figures ; il voit ses idées, et comme l'œil ne se voit point lui-même, nous concevons bien par là pourquoi et comment il est toujours si peu propre à la réflexion. Dans un aveugle enfin qui médite sur les formes solides ou les combinaisons numériques de points palpables, c'est le toucher qui est encore en exercice et qui participe peut-être aux fatigues ${ }^{1}$ de la méditation et du rappel.

Tels sont les instruments grossiers mais indispensables, je crois, à l'exercice de nos actes et de nos vouloirs les plus intellectuels; nous ne nous en apercevons guère plus, actuellement, que de la nécessité des signes tirés de ces instruments mêmes (et nous en savons bien la raison).

entre le domaine de la pensée et celui de la nature extérieure. Ces deux grands hommes se lancent des traits perdus, il faut se placer entre eux pour les ramasser.

1 Cette dernière observation appartient à Diderot. (Voyez sa lettre sur les aveugles.) Je n'en conclurai point avec lui que la pensée est alors au bout des doigts, mais qu'elle a réellement besoin d'y prendre un point d'appui pour s'exercer régulièrement à se réfléchir elle-même. 


\title{
Chapitre V
}

\section{Ordre actif intellectuel}

\author{
$2^{e}$ et $3^{e}$ classes. (voyez le tableau.)
}

Mémoire des opérations intellectuelles en particulier. Acte de comparaison et liaison des jugements. Du raisonnement considéré comme opération et des divers actes intellectuels qui le composent.

Retour à la table des matières

L'être pensant peut bien sentir, percevoir, imaginer ou se représenter les autres existences sans signes institués ou convenus avec lui-même ; mais en parlant et s'entendant, il double réellement l'image ou l'impression quelconque liée au son articulé ; il aperçoit, il pense. En communiquant donc le caractère d'aperception directe et simple à tous les modes en général, les signes volontaires (et ceux de la voix bien éminemment) impriment celui d'aperception réfléchie à ces modes originellement actifs en particulier, qui constituent le premier ordre de facultés ou d'opérations intellectuelles. C'est sous cette forme composée seule qu'il nous est donné d'en prendre connaissance, de nous en faire des idées individuelles. C'est ainsi que la pensée peut se mettre en regard d'elle-même, s'adresser des questions telles que la proposée, et tâcher d'y répondre, tantôt en se concentrant en elle et cherchant comment, et sous quelle modification elle se trouve pleinement constituée, tantôt en regardant au dehors pour se réfléchir spéculairement dans les objets de ses représentations ou dans ses organes, pour connaître les instruments et les moyens de son exercice ; mais c'est surtout la connaissance par concentration qui appelle les secours de signes oraux. 
Les termes particuliers que nous rattachons en effet à chacun de nos actes intellectuels, séparément de leurs résultats sensibles ou des perceptions passives avec qui ils s'agrègent, les empêchent d'aller encore s'y confondre, s'y absorber entièrement; c'est, comme nous l'avons dit, une barrière opposée à la pente de l'imagination et des habitudes; plus de tels signes sont multipliés lorsqu'ils correspondent d'ailleurs à des actes réellement distincts, plus nous avons de facilité pour étudier et connaître notre pensée (et sans doute il est bien moins dangereux ici de trop diviser dans les termes individuels que de trop composer dans les classes générales); c'est en évaluant ces formules sentir, percevoir, rappeler, penser, etc., par un retour plus intime dont les signes mêmes nous fournissent l'occasion et les moyens, c'est en prenant ces derniers sons sous leur véritable titre, ou comme signes d'opérations déterminées en elles-mêmes, et non pas seulement comme termes de valeurs composées représentatives qui s'adressent à l'imagination, c'est par là, dis-je, que nous prenons une connaissance claire de ce qui est dans le domaine propre de notre intelligence. Tels sont nos moyens d'analyse, et la personnalité elle-même, ce moi si sujet à l'envelopper ${ }^{1}$, combien sa propre conception réflective ne tientelle pas à la valeur individuelle que nous donnons à son signe expressif ${ }^{2}$ ?

Les fonctions, que les signes remplissent dans les idées purement réfléchies de nos opérations, doivent donc être bien distinguées de toutes les autres idées d'un ordre quelconque, et c'est en effaçant complètement cette distinction que l'on a été conduit à assimiler le raisonnement ou l'analyse métaphysique avec les plus simples déductions abstraites qui peuvent se fonder sur les méthodes

\footnotetext{
On ne saurait donc le considérer comme inné à lui-même ainsi que l'entendait Leibnitz.

Tout langage ne peut se fonder que sur la réflexion originelle, mais l'institution des signes réagissant sur la faculté institutrice lui rend ensuite progressivement bien plus qu'elle n'en a reçu. « À l'origine des langues, dit SMITH (voyez ses Considérations sur les langues), il est très probable que les pronoms personnels n'étaient pas connus, quoique l'usage nous les ait rendus familiers ; ils expriment une idée très abstraite et très métaphysique. Le mot $j e$, par exemple, est un mot d'une espèce tout à fait particulière ; il n'indique pas, comme le mot homme, une classe particulière d'objets, séparée de toutes les autres par ses qualités personnelles et propres ; loin d'être le nom d'une espèce, il désigne au contraire, lorsqu'on s'en sert, un individu précis, la personne qui parle ; il peut être regardé à la fois comme mot propre et mot commun. »

Ceci peut s'appliquer à tous les termes expressifs de nos actes intellectuels. Les prendra-ton sous l'acception propre et individuelle? on aura une analyse réfléchie et une simple énumération; sous l'acception générale ou commune, comme on le fait ordinairement pour la sensation, par exemple, on aura une analyse logique, il faut opter.

" Ce mot, continue Smith, exprimant donc une idée très métaphysique, ne devait pas s'offrir promptement aux premiers inventeurs des langues. Il est facile d'observer que les pronoms personnels sont au nombre des premiers mots dont les enfants apprennent à. faire usage. »

Cette séparation et distinction totale des pronoms demandent en effet une réflexion assez complète, exclue par la prédominance de la sensibilité et de l'imagination dans les premiers âges de la vie, comme dans l'enfance des sociétés, aussi ces pronoms sont-ils restés confondus avec le verbe dans les langues en particulier les plus favorables à l'exercice de l'imagination.
} 
mécaniques. Mais par cela seul que les procédés du raisonnement ou de l'analyse, seraient dirigés par des méthodes mécaniques, ils cesseraient d'appartenir (dan mon sens) à la métaphysique ; ils lui seraient diamétralement opposés et les résultats hypothétiques ou conditionnels obtenue par cette voie, n'ayant pas de moyen de vérification intérieure, ne sauraient donner la solution d'aucun problème de cet ordre ; quels qu'ils pussent être, ils n'entameraient même pas, pour ainsi dire, les questions posées dans un point de vue réfléchi.

Ce qui distingue le raisonnement qu'on pourrait appeler métaphysique, ce sont les fonctions que remplit la faculté que nous avons précédemment caractérisée sous le nom de mémoire intellectuelle. C'est ici qu'elle jouit sans restriction du titre que lui donna l'ancien philosophe cité auparavant (ex rebus mente conceptis "propositum») titre qui constitue en effet la mémoire intellectuelle, dans son rapport exclusif à la réflexion à qui elle redit (ou qui se redit plutôt à elle-même) les actes ou jugements antérieurs. Elle peut être dans son plein exercice, là précisément où toute représentation cesse par la nature même des choses, et ne peut continuer sans entraîner certaines illusions bien communes, qui mettent ce qu'on ne cherche pas à la place de ce qu'on poursuit, et font penser à une chose en parlant d'une autre ; là enfin où il ne devrait pas y avoir de mémoire possible, suivant les définitions reçues.

C'est dans cet ordre de surcomposés ou cet ensemble d'opérations comprises par les métaphysiciens sous le titre général de raisonnement, que nous achèverons de déterminer le caractère et les autres modes d'exercice de cette faculté de rappel, qui est elle-même le premier mobile d'activité de tout raisonnement développé dans les formules de nos langues usuelles ou scientifiques. Mais cherchons d'abord dans notre point de vue les raisons de ce nouvel ordre d'opérations, dont le titre propre et complexe se trouve produit ici pour la première fois, quoique les éléments se trouvent bien renfermés dans les analyses antérieures, sous des dénominations plus particulières.

\section{I. - Acte de comparaison. Origine du raisonnement.}

\section{$\underline{\text { Retour à la table des matières }}$}

Si par un premier jugement, dit Condillac dans sa Logique, je connais un rapport, pour en connaître un autre j'ai besoin d'un second jugement. La réflexion n'est qu'une suite de jugements qui se font par une suite de comparaisons. Le terme réflexion est pris ici dans un sens figuré ou purement métaphorique, pour exprimer l'attention de l'esprit qui réfléchit ou rejaillit en quelque sorte de dessus une idée ou perception partielle sur une autre ; mais en 
écartant toute métaphore et conservant aux mots, autant qu'il est possible, leur valeur propre, on trouverait alors que Condillac a très nettement caractérisé, sous le titre de réflexion, la faculté, qu'il exprime ultérieurement et en résultat, seulement logique, sous le titre de raisonnement.

Un raisonnement effectué, exprimé par des mots dans une formule du langage, consiste bien dans une suite de propositions ou de jugements liés entre eux et à un premier dont tout dérive. Les signes passagers ou permanents nous retracent bien les résultats des opérations successives de l'esprit, qui les a produits ou conçus ; ils nous font répéter ou imiter nous-mêmes ces opérations, mais les résultats ne sont pas les actes intellectuels mêmes, et ici nous reconnaissons la pauvreté du langage métaphysique, qui n'a jamais qu'un seul mot pour exprimer la faculté agissante et le produit qui lui est devenu en quelque sorte étranger et comme extérieur. La faculté se définit elle-même dans la réflexion, seule de son exercice; le résultat s'analyse au dehors dans diverses espèces de monuments, que l'activité de l'intelligence s'élève et qui aussi lui survivent ou la remplacent. De là diverses méthodes artificielles, sorte de formes sensibles dans lesquelles les signes se combinent, se lient ou se déduisent les uns des autres, quelquefois indépendamment des idées, etc., toutes choses qui ne constituent point l'opération de raisonner, laquelle réside non dans des jugements liés entre eux, comme par hasard, mais avant tout dans des jugements qui se font par une suite de comparaisons.

Or, la comparaison est un acte de la volonté qui se rend présente, sous cette même forme que nous avons nommée attention, à deux résultats simultanés ou rapidement successifs ${ }^{1}$. C'est la perception d'un rapport composé ou l'acte même qui prépare cette perception complète; mais avant le composé, est le simple, avant la proportion, est le rapport, avant l'attention double est l'acte unique de réflexion et d'attention simultanées, qui fait la conscience de l'idée simple ou individuelle. De même qu'on ne peut comparer deux nombres composés quelconques, sans former une véritable proportion où l'unité entre comme antécédent nécessaire, ainsi chaque perception, ou idée que nous appelons simple, est un véritable rapport à l'unité subjective. Chaque jugement,

La simultanéité peut être dans les résultats du vouloir quoique la succession soit dans les actes mêmes; et c'est ainsi, je crois, qu'on peut résoudre cette difficulté élevée depuis longtemps par les métaphysiciens et qui, n'en étant plus une pour nous, paraît pourtant subsister encore dans une école célèbre (dans l'École d'Édimbourg. Voyez ce qu'en dit M. Prévost, de Genève, dans une note de l'ouvrage déjà cité à l'occasion de M. Dugald Stewart). On demande si le principe pensant peut avoir plusieurs idées présentes, ou exécuter plusieurs opérations à la fois. Dans l'ordre des facultés passives, je ne crois pas que l'on puisse former de doute sur la simultanéité réelle de plusieurs modifications ou affections sensitives. Il en est à peu près de même dans les résultats des actes qui persistent encore après que la volonté a cessé d'agir, en vertu d'une vibratilité propre aux termes organiques, sur qui cette force se déploie directement ou indirectement. Sans une telle persistance, la comparaison même ne semblerait pas pouvoir s'effectuer, ce qui n'empêche point que les déterminations des actes demeurent successives. 
fondé sur un acte de comparaison ou attention double, est une véritable proportion dans laquelle cette unité constante peut être tout à la fois antécédent et conséquent.

\section{II. - Caractères divers de l'opération de raisonner ou de déduire suivant les différentes espèces d'idées associées et les modes d'association des idées entre elles ou à leurs signes, ou de ces signes entre eux.}

$\underline{\text { Retour à la table des matières }}$

En prenant la faculté de comparer ou de raisonner jusque dans ses éléments, il serait difficile de dire, à quel point rapproché de l'origine même de toute perceptibilité cette opération se rattache ; à peine pourrait-on la séparer de nos idées actuelles les plus directes en apparence, où l'existence des choses, des propriétés et qualités qui se dérobent aux sens, est affirmée ou conclue intérieurement de telle impression simple présente, par une chaîne plus ou moins longue d'images ou de souvenirs confus, parcourue avec toute la rapidité de nos plus intimes habitudes. Il serait très difficile, dis-je, de séparer ce qui est dans notre esprit, sous forme de déduction, des impressions qui semblent venir passivement affecter le sens ${ }^{1}$, tant l'habitude entée sur la nature même a fortement cimenté les éléments de ces premières idées sensibles, maintenant si composées.

Mais dans un second travail de nos facultés actives et dans une seconde création de ces produits alors proprement intellectuels et réflectibles par l'emploi des signes institués, les premiers agrégats de la sensibilité ou de l'imagination se résolvent dans leurs éléments recomposés sur un nouveau plan; ils s'approprient à une nouvelle connaissance; il s'agit de donner un ordre successif à ces termes élémentaires simultanés ou confondus en un seul, pour acquérir une perception distincte de chacun individuellement, de ses rapports avec tous les autres, et s'assurer enfin de l'idée complète de l'ensemble, ce qui ne peut se faire dans tous les cas, que par une suite d'actes de

Dans un ancien Traité sur les animaux, LACHAMBRE, médecin de Louis XIII distingue avec beaucoup de sagacité, une sorte de raisonnement qui s'effectue dans l'imagination même, dans les diverses opérations de l'instinct. C'est ainsi que l'habitude elle-même raisonne; ôtez la perception des actes effective ou possible, tout paraîtrait bien égal en effet dans les deux ordres de déduction. 
comparaison, au moyen desquels les jugements partiels se lient entre eux, depuis celui qui affirme la qualité la plus simple, jusqu'à celui qui les enveloppe tous dans une même existence ou sous un seul signe. Toute décomposition ou recomposition régulière d'un ordre quelconque, peut donc être dite raisonnement, de quelque manière et par quelque mobile que la suite des opérations s'effectue.

Mais suivant la nature diverse des idées ou des termes associés, suivant que les caractères sensitifs, perceptifs, aperceptifs, réfléchis ou purement abstraits dominent dans les éléments du composé, il est bien facile de voir que ce ne seront ni les mêmes facultés en exercice, ni les mêmes mobiles qui les activent, les mêmes moyens qui les appliquent ou les dirigent. Et quoique le raisonnement effectué ou développé dans un certain ordre d'analyse ou de synthèse, suivant certaines formes logiques, ne soit dans tous les cas qu'une suite de jugements liés entre eux, on conçoit que la diversité des éléments liés, jointe à celle de l'exercice particulier de la puissance qui opère la liaison, ne permettront pas d'affirmer l'identité réelle du fond, d'après la similitude apparente des formes, ni d'appliquer aux différentes espèces du raisonnement ce qui se trouve convenir au genre exclusivement établi sur l'analogie de ces formes artificielles.

S'agit-il en effet de composer un archétype imaginaire ou de rassembler dans un objet fantastique les qualités éparses dans plusieurs ${ }^{1}$, nous avons vu avec quelle spontanéité agit la faculté, qui exécute ces rapprochements, ce n'est pas la composition qui est laborieuse en ce cas, c'est l'analyse à froid qui ne saurait retrouver dans le tableau tous les éléments qui y sont entrés, et laisse échapper précisément ceux qui en font la vie ${ }^{2}$; on analyse ce qui se conçoit ou se représente, mais non pas ce qui se sent.

Or, dans ce premier travail seul exécutable, ce n'est plus la mémoire qui, sous son véritable titre, fournit les éléments associés dans un nouvel ensemble propre à frapper ou émouvoir, ou du moins son exercice y est bien subordonné ; l'attention est commandée par la vivacité du tableau, et dans ce qui tient à l'appréciation calme et réfléchie plutôt qu'à un sentiment immédiat

Car c'est là aussi une sorte de raisonnement, et voilà pourquoi Condillac, faisant abstraction du mobile d'activité, ne considère l'imagination même, que comme un exercice particulier de la réflexion, c'est-à-dire, dans notre sens, du raisonnement.

2 Parce que toutes les idées que Locke appelle modes mixtes, et qu'il a considérées trop généralement comme archétypes ou sans modèle, se trouvent toujours associées à quelques sentiments plus ou moins profonds, que leurs termes ont acquis, par une ancienne habitude, le pouvoir de réveiller. C'est, ce me semble, mal connaître leur nature et ne tenir aucun compte des éléments principaux dont elles se composent, que de prétendre leur appliquer la même espèce d'analyse, les soumettre aux mêmes formes du raisonnement et de démonstration rigoureuse, que les idées abstraites des quantités et des opérations dont nous sommes les auteurs réfléchis. 
ou sympathique des rapports, l'analogie naturelle des images prépare encore une liaison facile des jugements.

Lorsque le raisonnement a pour but de décomposer ou recomposer de nouveau une idée dont le premier modèle est donné par les sens perceptifs ou aperceptifs, l'opération s'accomplit et se vérifie encore par un travail plus ou moins facile. La mémoire exerce là la fonction représentative; elle rappelle les signes des qualités abstraites sensibles, suivant l'ordre de la représentation même. La réflexion inséparable de tout exercice de la mémoire (proprement dite) entre sans doute dans ce travail, mais elle ne peut y prendre qu'une faible part; l'attention, soutenue par l'attrait naturel attaché aux représentations claires des sens, y trouve un exercice facile, et c'est par cette faculté, sous le nom de comparaison, que s'opère la liaison des jugements qui n'est point séparée alors de celle des images mêmes.

Mais lorsque l'opération du raisonnement est tout entière dam la comparaison d'idées abstraites complexes ou réflectibles, qui ont toute leur existence et leur appui nécessaire dans les signes institués (et c'est le cas que nous avons ici plus particulièrement en vue), l'imagination ne fournit plus d'appui ou de mobile d'activité à l'attention, ni de matière aux actes de la mémoire, tout passe sous un autre domaine. L'organe intellectuel livré, pour ainsi dire, à ses propres efforts, doit tout tirer de son sein; les signes n'étant plus qu'indicateurs des opérations dont ils résument et conservent les résultats, il s'agit d'abord, pour leur donner un sens, d'effectuer ces opérations mêmes ; confiés ensuite à la mémoire, le rappel des termes, s'il n'est pas tout à fait vide d'idées devra toujours être accompagné d'un retour plus ou moins profond sur les actes exprimés, et dans ce souvenir tout est à la réflexion, rien à la représentation. Ce dépouillement d'idées sensibles, cette rupture du commerce extérieur étonne d'abord l'esprit, révolte les premières habitudes, mais bientôt familiarisé avec la nudité des signes, il y trouve des moyens faciles et commodes, pour atteindre, même sans le travail de la réflexion, tous les résultats et les rapports qu'il peut se proposer dans ce nouveau monde de ses idées.

La mémoire des signes qui indiquent seulement des opérations exécutées ou à exécuter sur certaines idées, en laissant le matériel de ces idées indéterminé pour l'imagination, cette mémoire, dis-je, en tant qu'elle nous retrace les mêmes actes intellectuels, se trouve bien constituée ici sous le véritable titre et dans son rapport à la réflexion; mais nous avons vu qu'elle se borne souvent à nous assurer que ces actes ont eu lieu, alors il ne reste plus, pour ainsi dire, que leurs signes matériels, sur le témoignage affirmatif desquels la pensée se repose. Il ne s'agit plus que de savoir si les formes sont exactement observées, si les termes se suivent dans leur ordre, et la mémoire, chargée de les retracer ainsi, n'ayant presque plus qu'une fonction mécanique à remplir, le raisonnement devient alors bien facile. 
Dans la liaison régulière de nos idées et de nos jugements sur l'existence des choses ou des faits de tout ordre, il y a toujours une vérification et une double comparaison à faire, des signes aux idées et de celles-ci aux objets ou aux modèles donnés par le sens externe ou intérieur (réfléchi); bien voir ou bien entendre ce qui est au dedans ou au dehors et le peindre ou l'exprimer avec précision et fidélité, telle est la double tâche de l'observateur.

Mais lorsque nous sommes en présence des idées ou des termes qui tirent toute leur valeur de nos propres conventions et qui sont de véritables archétypes dans l'ordre abstrait, il n'y a plus de modèle à consulter, et il ne s'agit que d'être fidèles à ces mêmes conventions que nous avons établies. Or, celles-ci peuvent encore être telles, que les signes soient chargés de nous rappeler différentes espèces d'idées ou d'éléments mixtes qui s'y trouvent associés, ou seulement des opérations, des vues de l'esprit, qui peuvent être, pour ainsi dire, empreintes dans ces signes, de telle sorte que par le simple fait du rappel de ceux-ci, ou par la seule observation de certaines formules mécaniques, les opérations soient exécutées infailliblement ; c'est ce qui a lieu, comme on sait, dans les raisonnements avec les signes abstraits de la quantité, et surtout ceux de l'algèbre. Ces raisonnements ne sont en effet que des transformations ou des traductions de signes d'opérations (et non point d'idées), depuis la forme la plus composée qui énonce le problème, jusqu'au résultat final et simple qui le résout. Dans ce cas la liaison des jugements est annoncée ou préparée par celle des termes, plutôt qu'effectuée et considérée dans ses procédés techniques, l'analyse algébrique est peut-être moins une méthode de raisonnement, qu'un moyen mécanique de parvenir à tel résultat, sans avoir besoin de raisonner ou de former une suite de jugements, à l'aide d'une suite de comparaisons.

Parce que les mathématiciens sont heureusement parvenus à se passer de raisonnement, on a substitué la liaison des signes à celle des jugements, sauf à rétablir ensuite celle-ci, quand ils connaissent le résultat. De ce qu'ils emploient enfin avec succès des méthodes mécaniques certains philosophes ont trop légèrement cru, qu'il serait possible d'introduire, sans danger, des méthodes semblables dans les déductions de nos idées mixtes de tout ordre. Ils ont trop cru qu'à l'exemple des géomètres il n'y avait qu'à bien arrêter d'abord ses conventions avec sa propre pensée, bien faire sa langue, pour se laisser aller ensuite aux transformations qu'elle indique, être autorisé à prendre toutes les proportions sur les signes, et à se débarrasser du fardeau des idées pour aller plus vite.

Parce que tout le raisonnement en mathématique se réduit à l'identité, comme tous les jugements à celui qu'on peut appeler de compréhension (puisqu'il ne s'agit presque jamais, en effet, que de bien connaître tout ce qui est renfermé dans les idées faites, ou les termes institués par nous, et qu'on est 
sûr de posséder avec ces termes tous les éléments et les combinaisons d'éléments homogènes qui ont concouru à les former), il ne s'ensuit point que la liaison de nos jugements d'observation extérieure ou intérieure soit exclusivement soumise à la loi d'identité, ni que tout puisse y être ramené à une seule forme de perception de rapport d'idées renfermées les unes dans les autres ou dans une première, en qualité d'élément circonstanciel; c'est bien là d'une part tout réduire aux classifications de langage, et d'une autre part ramener les différentes espèces de rapports que nous pouvons concevoir entre divers systèmes d'idées, à celui qui ne convient pas même exclusivement à l'ordre de notions abstraites, auquel il est pourtant le plus applicable; dans le cas, par exemple, où il ne faut rien moins que toute l'activité du génie ou la force de la réflexion, pour combler l'intervalle qui sépare deux idées ou deux faits de la nature, peut-on les considérer comme renfermés ou compris l'un dans l'autre, de même que 2 l'est dans 4, etc. ?

Ici, je sens le besoin de déterminer, avec toute la précision possible, en quoi consistent ces deux lois de la liaison de nos jugements (celle d'identité et de compréhension), que de grands maîtres s'accordent à reconnaître comme générales et exclusives. Je chercherai à interpréter le sens et le fondement de ces lois, par des applications à des exemples simples, qui pourront jeter quelque jour sur le mécanisme du raisonnement et les opérations relativement involontaires qui y concourent. Je chercherai, dis-je, à éclaircir ces questions pour moi-même, déclarant ici, encore plus expressément que partout ailleurs, que je suppose des doutes, j'étudie, et ne prétends point faire autorité ; je vais donc d'abord reprendre d'un peu plus haut le fondement de cette idée d'identité et de compréhension.

\section{III. - Recherche sur le fondement des conceptions d'identité et de compréhension.}

\section{$\underline{\text { Retour à la table des matières }}$}

Nous avons le sentiment de l'identité du moi sous des modifications diverses. Ce sentiment, inséparable de la puissance d'action ou d'effort, s'étend par une liaison première et nécessaire, et du dedans au dehors, aux termes constants sur qui la même force se déploie. C'est ainsi que nous reconnaissons l'identité des substances étrangères. Ôtez l'action d'une force constante ou le mode relatif d'effort, nous ne concevons plus de fondement à la notion d'existence identique en nous, ou hors de nous. 
Nos modifications, en se répétant, ne sont point reconnues identiques par elles-mêmes, mais seulement dans les causes subsistantes, auxquelles nous les rapportons par l'habitude d'un jugement premier, et presque toujours il nous arrive de transporter à l'effet senti l'identité de la cause aperçue ou supposée.

Les images plus ou moins confuses, les vues spontanées et fugitives de notre esprit, peuvent toujours se rallier à quelques signes qui sont encore des termes ou des produits de l'action que notre volonté crée. Il ne serait pas trop hardi peut-être, d'affirmer que, hors de l'association à ces signes, les mêmes modes passagers pourraient se produire intérieurement, sans qu'il y eût aucun type fixe de leur identité.

Dans toutes les conceptions quelconques, qui ne représentent point directement des objets, tout le mobile de la réminiscence est bien évidemment dans le signe. Prenons, par exemple, les idées les plus simples de la quantité, qui n'ont aucun titre réel hors de leur terme déterminatif.

Que je donne un signe à l'unité, et que je le répète indéfiniment en cette sorte : $1+1+1$, etc., je ne conçois jamais qu'une seule idée numériquement individuelle, parce que je ne produis qu'un même signe, un même acte, mais que j'attache des termes différents à chaque collection progressivement croissante de l'unité, j'aurai autant d'idées distinctes que de termes ; en répétant chacun d'eux en différents temps, j'ai la conscience de l'identité de l'opération qu'il exprime, tandis que les idées de divers nombres demeurent parfaitement séparées dans mon souvenir.

Lorsque je dis $1+1$, ou que j'ajoute 1'unité à elle-même, j'ai deux idées identiques, ou plutôt une même idée reproduite deux fois dans mon esprit ; le signe dont je me sers pour indiquer cette répétition n'est point celui de l'idée même, il doit en être bien distingué. Lorsque je réunis les deux unités sous un seul terme 2, ce nouveau signe indique une autre opération et même une autre idée ; il n'y a pas en effet d'identité réelle entre la conception de deux objets isolés dans l'esprit, et celle des mêmes objets réunis et comme fondus en un seul tout pas plus qu'entre les signes 1,1 et 2 qui expriment ces deux points de vue; mais le terme 2 comme la formule $1+1$ ont pour fonction égale de représenter ou de rappeler à mon esprit deux choses qui, soit unies, soit séparées sont toujours deux dans la quantité ; c'est cette égalité de fonction, que j'exprime par la formule équationnelle $1+1=2$, qui n'est point du tout la même que l'équation vraiment identique $2=2$; cette dernière n'apprend rien, la première est une analyse ou une définition.

En continuant de former les collections de la même unité, je dis encore $1+$ $2=3$, ou $1+1+1=3$ et j'ai encore trois opérations et trois idées différentes, comme si je figurais des impressions de cette manière $1,1,1$ puis $1(1+1)$ qui marque la réunion de deux parties et la séparation de l'autre, puis enfin 
$(1+1+1)$ qui les réunit en une seule idée. Ces trois points de vue sont réellement autres, mais les trois expressions ont encore pour fonction égale de marquer une valeur triple constante, ou une même idée reproduite trois fois, quel que soit l'arrangement ou la forme que je donne à cette répétition considérée dans le signe ou dans l'objet. Je conclurai de là que l'identité n'existe point, du moins n'est jamais que partielle dans les vues de l'esprit, lors même qu'on se croit, le plus en droit d'affirmer qu'elle est absolue, et que l'égalité de fonction entre deux signes qui expriment une idée considérée sous deux rapports différents, ou deux idées considérées sous un même rapport, doit toujours être bien distinguée d'une identité réelle qui suppose à la fois le rappel d'un seul et même acte et d'une seule et même idée, que la permanence du signe fait reconnaître les mêmes dans leur répétition indéfinie.

En ne considérant que le nombre, on peut donc dire qu'il y a, non identité, mais égalité absolue de fonction entre deux signes ou formules, qui peuvent être substituées l'une à l'autre dans un calcul, le résultat demeurant le même ; ainsi je puis substituer $7+2$ à $5+4$ et remplacer ces deux formules par un seul signe 9, et il n'y aura rien de changé dans le nombre ou la quantité totale résultante, quoique les vues de l'esprit soient réellement distinctes et que les expressions ne puissent être dans plusieurs cas, prises indifféremment l'une pour l'autre.

Lorsque j'observe pour la première fois la propriété commune, qu'ont ces deux formes $7+2$ et $5+4$ de donner exactement une même valeur 9 , je fais une découverte dans mes idées, je perçois l'égalité et non l'identité de deux rapports, car l'identité aperçue ne peut jamais être une découverte, ce n'est qu'une reconnaissance. Dans toutes idées autres que celles de la quantité nombrable, on ne peut jamais à plus forte raison substituer un signe à un autre en conservant l'identité d'idée ou de rapport (nous ferons voir bientôt qu'il n'y a même pas lieu à égalité) et comme l'ont très bien vu de judicieux philosophes, il n'existe point de parfaits synonymes dans aucun système de nos idées mixtes.

Quand il ne s'agit que des idées de quantités homogènes dans leurs éléments, l'ordre de composition de ces derniers ne change rien à la nature du résultat total, considéré sous le rapport unique de son extension ; que l'unité ait été simplement ajoutée un certain nombre de fois, ou qu'on ait pris telle de ses collections déterminées quelconques, pour composer le même nombre, la quantité résultante sera exactement la même; mais dans nos idées mixtes de tout ordre il serait possible que les éléments fussent les mêmes en nombre et en qualité, et qu'il n'y eut aucune identité entre les composés qui s'en forment, si ces éléments variaient dans leur nature intensive, si leur ordre, leur proportion changeait, si quelques-uns d'entre eux avaient dans tel composé, auquel ils concourent, une primauté ou une prédominance qu'ils n'ont plus dans un autre produit mixte, dont le langage exprime pourtant l'idée constante. 
Le signe numérique ou permanent dans sa forme matérielle visible ou audible, rappelle bien une opération identique, et qu'il dépend de nous d'effectuer toujours de la même manière. Voilà pourquoi la réminiscence, inséparable du signe, tient lieu parfaitement ici de l'opération première et complète. Toute combinaison de signes, qui exprimera ou rappellera le même résultat, pourra être dite absolument égale en nombre et en valeur à une autre quelconque, dont la fonction sera de reproduire la même quantité ; ainsi l'expression ou idée $(7+2)$ n'est sûrement pas identique à celle de $(5+4)$, mais en tant qu'on les rapporte à la même collection 9 , il y a égalité parfaite de fonction, et c'est ce qu'on exprime en formant l'équation $7+2=5+4$, conclue de $7+2=9$ et de $5+4=9$.

Dans nos autres idées composées d'éléments divers, et que nous n'avons point faites ni pu faire nous-mêmes, le même signe, en se répétant, peut bien ne pas correspondre dans notre esprit à la même idée, et c'est le plus souvent une grande illusion de transporter au caractère intrinsèque de cette dernière, l'identité qui n'appartient qu'au terme propre du rappel; il ne s'agit point ici seulement de rappeler un signe et de répéter l'opération dont il exprime nettement le résultat, mais il faut savoir de plus, si l'élément sensible ou objectif de l'idée, la matière dont l'acte intellectuel est la forme, a conservé cette permanence qui lui est attribuée par confusion avec la partie formelle agrégée ou combinée. Or, nous avons la preuve que ce premier élément varie sans cesse par sa nature dans une multitude de cas, et presque toujours il est impossible de vérifier et d'assurer hors du signe une telle identité.

Lorsque deux expressions différentes sont supposées correspondre au même terme ou à la même idée complexe, c'est toujours en tant que cette idée appartient à deux idées différentes rapportées à une même classe, ou que les deux signes expriment, sous un fond commun, quelques nuances particulières ou quelques circonstances accessoires qui se trouvent en plus ou en moins, dans les deux composés. Dans le premier cas, nous formons une suite d'équations logiques entre chacune des deux idées composées et une troisième qui est censée renfermer leurs communs éléments, les embrasser également toutes deux dans leur extension, et être comme la mesure partiellement exacte de l'une et de l'autre. Ainsi nous disons que l'homme est un animal, que le lion est un animal, à peu près comme nous dirions $7+2=9,5+4=9$; mais dans ce dernier cas nous concluons l'égalité absolue des deux premiers membres de l'équation, parce que nous n'avons égard qu'au rapport du nombre des éléments identiques. Dans le second, cette égalité ne peut avoir lieu, parce que outre la somme des idées ou qualités partielles prises séparément dans chaque idée totale spécifique ou individuelle pour former le même genre animal, il est resté encore dans chacune de ces idées totales génératrices plusieurs éléments divers, qui ne sauraient entrer en équation. Le terme identique, appliqué également aux deux espèces subordonnées, n'embrasse que le nombre des éléments qui ont été extraits de toutes les deux pour le former, et laisse toute 
l'autre partie différentielle, absolument indéterminée et sans expression; il n'y a donc point là d'identité dans aucun sens. Je dis de plus qu'il n'y a point d'égalité de fonction dans le signe général, considéré sous le rapport de son extension commune aux deux idées spécifiques différentes qu'il embrasse par convention, en n'ayant égard qu'au nombre des éléments extraits de ces idées. Nul doute que le signe de l'idée générale ne convienne également à l'une ou à l'autre, lorsqu'on les considère sous le rapport de tel nombre de qualités abstraites ; mais comme ces qualités ou propriétés, quoique exprimées par les mêmes termes, peuvent différer singulièrement par leur intensité, et que les degrés ne sont point susceptibles de mesure, on voit bien que l'équation logique ne subsiste que dans la forme, et que l'identité du signe peut devenir très illusoire. L'idée d'animal étant formée de celles de divers phénomènes, par exemple, sentir, se mouvoir, se nourrir, croître, se propager, etc., convient également aux espèces de l'homme et du lion, quant aux nombres des éléments qui font partie de ces deux dernières collection partielles ; mais il n'y a point identité entre chacun de ces éléments ni par conséquent entre les deux collections partielles qu'exprime le mot animal, abstraction faite même des autres éléments différentiels non exprimés; soit par exemple, l'idée complexe exprimée par le terme homme, analysée de cette manière : $s+m+n+c+\ldots+$ $p$ (en ne prenant pour abréger que les lettres initiales des propriétés composantes, sentir, se mouvoir, etc.) ; l'idée spécifique de lion étant exprimée par la formule $s^{\prime}+m^{\prime}+n^{\prime}+c^{\prime}+\ldots+p^{\prime}$, si j'extrais de ces deux formules les éléments semblables et identiques par l'hypothèse pour en composer une seule idée, je ferai $s+m+n+c=$ A et $s^{\prime}+m^{\prime}+n^{\prime}$, etc. $=\mathrm{A}^{\prime}$ Alors suivant l'identité logique ou supposée par la similitude ou l'analogie des formes, j'étendrai à $A^{\prime}$ tout ce que j'affirme de $A$, et effaçant leur caractère en ne mettant pas plus de différence entre les idées A et A', que nous n'en mettons entre le même terme animal répété deux fois dans une proposition, je raisonnerai comme si $\mathrm{s}+\mathrm{m}+\mathrm{n}+\mathrm{c}$, etc. égalait s' $+\mathrm{m}$ ' $+\mathrm{n}$ ', etc., de même que $7+2$ égale $5+4$, parce que tous deux vont se résoudre dans la même idée d'un seul et même nombre, et ont un même rapport à l'unité ; je traiterai des qualités intensives comme des qualités extensives, je supposerai et j'affirmerai en moimême, par un résultat infaillible de l'habitude, l'identité des idées où je trouve établie celle des signes, et la liaison de ces derniers me tiendra lieu des actes de comparaison qui devaient seuls former le lien de mes jugements.

Bientôt peut-être, et lorsque mes idées surtout n'auront pas de modèle fixe en dehors, je subordonnerai la nature même des choses à mes classifications ou à mes conventions artificielles, car enfin si je n'ai besoin que de consulter la valeur des signes pour former l'équation $7+2=5+4$, pourquoi aurais-je besoin d'un autre secours, que de celui du langage ou de mes propres conventions, pour raisonner sur toute espèce d'idées, c'est-à-dire dans tous les cas pour réduire, au moyen de l'identité des jugements renfermés les uns dans les autres. 
Lorsque les signes n'expriment que des opérations ou des rapports, ils se confondent avec les choses signifiées, on peut alors attribuer sans inconvénient aux uns et aux autres les mêmes propriétés.

Lorsque plusieurs termes se contractent, pour ainsi dire, en un seul, comme +1 et 1 , dans 2 , où les signes 1 et 1 se trouvent compris ou inscrits dans 2 , comme si on les rangeait ainsi $1+1$, nous disons métaphoriquement qu'une idée est renfermée dans une autre ; c'est une véritable figure, calquée en grande partie sur les images de la vue. Nous disons aussi que les qualités secondes, qui sont réellement nos propres sensations, sont renfermées dans les objets, que les effets sont renfermés dans les causes; c'est ainsi que nous exprimons très improprement les divers rapports de liaison, de coexistence ou de dépendance de nos modifications et de nos idées entre elles et de leurs objets. Mais comme l'analogie n'est pas l'identité, toute liaison de dépendance ou de coexistence n'est pas compréhension; le rapport de la coexistence même suppose bien diversité et non pas identité.

Nous disons que deux impressions ou deux images sont liées entre elles, lorsque l'une a le pouvoir de réveiller l'autre, de telle manière que l'être sentant se trouve modifié par une seule, qui vient à se produire en lui, à peu près comme il le serait ou comme il l'a déjà été par leur ensemble. La disposition, que deux modes quelconques ont à s'associer ainsi, tient souvent à une certaine analogie naturelle des impressions, dont la cause est cachée dans notre intime organisation, quelquefois dans le consensus sympathique des parties impressionnables, etc. En vertu de cette analogie, l'individu qui passe d'une modification à celle qui suit, sait à peine que son état est changé, et si les modes sont de nature à être comparés, il affirmera en lui-même la ressemblance des effets et peut-être l'identité des impressions ou de leurs causes. La même liaison peut dépendre aussi des habitudes ou de la répétition fortuite des mêmes impressions successives ou simultanées; le résultat est à peu près le même.

De telles associations se forment en nous de très bonne heure, et précèdent même la connaissance. Il s'en établit aussi, pour ainsi dire, à notre insu; dans toutes les époques de la vie; ces associations existent alors dans la sensibilité ou l'imagination, et non point dans le jugement; pour qu'elles puissent être dites dans le jugement, il faut qu'elles soient remarquées, ou que l'individu ait conscience de deux manières d'être, qui se suivent ou s'accompagnent; alors elles sont, pour lui en liaison ou en dépendance l'une de l'autre ; on ne saurait dire qu'avant cette conscience double, le jugement soit renfermé dans les impressions, pas plus que nos sensations elles-mêmes ou bien les vues de notre 
esprit, les abstractions sensibles, ne sont renfermées dans les objets, avant que nous ayons éprouvé les unes, ou eu l'occasion de former les autres ${ }^{1}$.

La faculté d'aperception ne s'applique directement qu'aux actes de la volonté ou à leurs résultats; les associations des modes actifs sont donc véritablement dans notre conscience, ce sont des jugements; ces jugements se lient dans l'ordre successif, et se déduisent, pour ainsi dire, les uns des autres, suivant certaines formules naturelles ; mais des habitudes profondes changent cet ordre ; elles suppléent nos actes, en effacent le sentiment, ou si elles les laissent subsister, c'est en accélérant leurs séries, de manière que les extrêmes se touchent, les prémisses vont se fondre dans la conséquence et à la place d'actes ou de jugements multiples, liés entre eux par une suite d'actes de comparaison, il n'y a plus en apparence qu'un résultat sensible unique. C'est ainsi que tous les jugements qui accompagnent nos sensations, lorsque nous rapportons de différentes manières les qualités aux objets, les impressions à leurs causes, les images à leur modèle, etc., nous échappent absolument et que nous ne distinguons plus les signes naturels des choses signifiées, les prémisses des conséquences; nous sommes portés à croire que les idées découlent du dehors par le canal des sens, faites de toutes pièces; c'est alors qu'on peut dire par une métaphore assez juste, que nos jugements et leurs suites sont renfermés ou enveloppés dans la sensation.

Les signes institués viennent briser cette enveloppe, rétablir de nouveau l'ordre successif, développer la chaîne concentrée de nos premières déductions, ou plutôt en renouer les fils; les étiquettes attachées à deux modes ou deux idées, que nous pouvons éprouver ou remarquer séparément, les empêchent à jamais de se confondre ; ces signes mis à côté l'un de l'autre suffiraient pour indiquer l'existence séparée des modes qu'ils expriment, ou le fait simple de leur liaison dans le sens ou dans l'imagination; mais il y a de plus un terme indicateur de l'aperception ou du sentiment que nous avons de cette liaison et dès lors celle-ci se trouve arrêtée d'une manière fixe pour le jugement. L'analogie ou la convenance de deux idées, qui fait qu'elles s'accompagnent ou sont en rapport de dépendance aperçue l'une de l'autre, est exprimée dans une formule qui comprend nécessairement trois termes; les jugements se lient entre eux, et l'un conduit à l'autre par l'analogie ou la dépendance des idées, comme l'une de ces idées séparées a le pouvoir d'en réveiller une autre. L'individu aperçoit cette liaison des jugements, il l'opère même par l'exercice de son activité, et il l'exprime par de nouvelles formules, où les logiciens reconnaissent et analysent le matériel de raisonnement.

L'erreur des platoniciens qui réalisaient les idées en les plaçant hors de l'entendement même, dans une région mystérieuse, parait être bien loin de nous ; et cependant toutes les formes de notre langage consacrent cette réalisation absolue des produits intellectuels, comme s'ils persistaient hors de l'esprit qui les conçoit. 
Un sens peut être frappé de plusieurs impressions à la fois ; diverses qualités peuvent se rapporter au même objet; un même tableau imaginaire est souvent composé de plusieurs parties; dans l'institution des signes un seul devra donc se trouver d'abord attaché à une grande variété et multiplicité de modifications ou d'idées ; il ne renfermera point, il est vrai, ces modes qui n'ont pas encore été remarqués, mais lorsqu'ils le seront et dans une seconde institution réfléchie du signe, il aura acquis le pouvoir de réveiller toutes les idées ou circonstances collatérales, que l'attention aura séparément fixées. La liaison des idées partielles sous une seule idée, ou de plusieurs termes élémentaires sous un seul terme complexe, lorsqu'elle a été formée par l'attention successive donnée à ces éléments, peut toujours déterminer une suite de jugements ou de propositions liées entre elles; c'est ainsi que le terme s'analyse ou se recompose, en faisant seulement un retour sur la valeur des signes, s'il s'agit d'une idée abstraite complexe de notre création, et en comparant de plus l'idée à un mode qui est ou existe donné par les sens ou la réflexion, s'il s'agit d'analyser un fait extérieur ou intérieur.

Dans cette analyse ou cette série régulière des déductions, nous pouvons souvent substituer, à mesure que nous avançons, un terme sommatoire partiel à tel nombre d'éléments qui lui équivalent, en reconnaissant l'égalité de valeur, et c'est ainsi que nous arrivons par une suite de substitutions, depuis le premier élément jusqu'au composé total par la synthèse, ou de l'idée totale jusqu'à l'élément générateur par l'analyse. Ainsi pour donner un exemple simple : partant de la décomposition de l'idée ou du terme 8 en $5+1+1+1$ j'observe que $1+1+1=3$ et substituant cette valeur, je conclus que $8=5+3$; c'est un raisonnement que l'on peut appeler synthétique ; j'aurais substitué au contraire $1+1+1$ à 3 si j'eusse été en rétrogradant depuis le terme le plus élevé par une suite de jugements qu'on pourrait appeler analytiques; nous avons vu ce qui empêchait de faire de telles substitutions rigoureuses, lorsqu'il s'agit d'idées autres que celles de la quantité.

Nous pouvons observer que toutes les transformations de l'analyse algébrique sont réductibles à de véritables syllogismes en formes, et qu'on n'y a peut-être pas encore assez vu tout le mécanisme du raisonnement; il faut en excepter les cas où l'on emploie des formules implicites ${ }^{1}$ qui ne peuvent absolument se traduire ; alors il n'y a point de raisonnement d'aucune espèce.

1 Un géomètre philosophe nomme formules implicites ces expressions telles que $\sqrt{-1}$, qui n'ayant en elles-mêmes aucune valeur déterminable sont susceptibles néanmoins, par des transformations ou des combinaisons avec d'autres expressions de la même espèce, de donner des résultats réels. C'est l'emploi de telles formules implicites(ou de tels signes vides d'idées) qui fait la caractère de l'analyse et la distingue de la synthèse ; celle-ci ne peut jamais perdre de vue son objet, il faut que cet objet s'offre à l'esprit clair et net, ainsi que tous les rapprochements et combinaisons qu'on en fait. La synthèse ne peut donc jamais employer de formules implicites, raisonner sur des quantités absurdes, sur des opérations non exécutables; les signes ne peuvent jamais être pour elle que de simples abréviations. 
La transformation faite du terme encore composé, dans sa valeur analytique, lorsqu'il s'agit de la résolution d'une idée complexe, ou de plusieurs termes élémentaires dans leur valeur synthétique, lorsqu'il s'agit de recomposer, se fonde d'abord sur la liaison des signes, qui donne lieu au rappel réciproque de l'un par l'autre.

\section{IV. - Fonctions diverses de la mémoire dans la liaison des jugements ou des signes.}

$\underline{\text { Retour à la table des matières }}$

C'est ainsi que la mémoire peut exercer différentes fonctions :

1. On substituera un terme à un autre, uniquement parce qu'ils se trouvent associés par l'habitude, sans qu'il y ait jamais eu conscience de l'égalité réelle de leur valeur ou du fondement de cette égalité. Dans ce cas, la mémoire est mécanique ${ }^{1}$, et il n'y a point de liaison de jugements, quoiqu'il puisse y en avoir un régulier de signes.

2. On peut se souvenir d'avoir nettement perçu le rapport d'égalité des termes substitués, ou de l'avoir arrêté soi-même dans les conventions du langage, mais sans le vérifier de nouveau; alors il y a rappel du signe et réminiscence simple de l'opération première.

3. Enfin il peut y avoir un retour complet sur la valeur des signes et les conventions qui les instituèrent; alors la mémoire est réfléchie ou n'est que la réflexion même unie à la réminiscence; alors aussi il y aura une véritable liaison de jugements et le raisonnement total s'établit sur une suite de comparaisons méthodiques entre les termes ou les idées (analysées jusque dans leurs fondements), ou entre ces dernières et leurs modèles, lorsqu'il s'agit de raisonnement sur les faits ${ }^{2}$.

Condillac n'a point envisagé l'analyse sous ce rapport, quand il la recommande comme la méthode exclusive, et s'il eût été jusque-là dans la langue du calcul, il n'eût pas cru que tous nos raisonnements étaient réductibles à des formes mécaniques.

Voyez le chapitre précédent.

2 Cet exercice de la mémoire réfléchie ne s'applique qu'à chaque terme partiel de la déduction, individuellement considéré ; car dans la série des jugements liés, dont se compose une analyse ou un raisonnement développé, il ne peut y avoir que réminiscence simple des termes ou actes qui précèdent celui que l'on considère, ou que saisit actuellement la vue intérieure. Jamais deux actes ne sont à la fois effectués et aperçus, mais chaque produit successif persiste, en résultat, dans l'organe intellectuel et celui qui vient 
4. Les transformations successives, par lesquelles s'effectue la liaison des jugements, ne sont point déterminées au hasard, il y a toujours un but présent ou éloigné que l'intelligence ne perd point de vue, vers lequel l'attention est fixée et c'est cette idée permanente d'un certain but ou terme final à atteindre, qui règle le choix des moyens, l'emploi des signes ou des formes de déduction les plus propres à l'atteindre avec certitude et célérité. C'est ce choix, cette comparaison continuelle des moyens au but, qui caractérise surtout la faculté de raisonnement; là est le complément ou le plus haut degré d'exercice de l'activité intellectuelle ; la suite des jugements liés est le résultat mais non la puissance.

La mémoire se charge bien de fournir avec chaque terme sa valeur analytique, mais le choix des éléments, la disposition ou l'ordre à donner à leurs collections partielles, pour que la série des jugements converge d'une manière plus directe vers la fin, supposent toujours un travail réfléchi qui se fonde bien sur la faculté du rappel, et que les habitudes agrègent et facilitent, mais qui ne peut non plus lui être exclusivement attribué. Quelquefois pourtant, et lorsqu'il s'agit surtout de déductions toutes nouvelles, le choix des moyens intermédiaires ou de l'espèce de transformation à adopter parmi plusieurs qui seraient également possibles, se fonde sur des comparaisons si rapides, sur une sorte de tact si prompt, sur des analogies si fines, que la réflexion même semble s'identifier avec l'imagination et prendre comme elle un caractère de spontanéité. C'est là qu'il faut reconnaître encore l'effet d'une sorte d'instinct ou de pressentiment de la vérité, qui emporte le génie hors de la ligne de méthodes, lui ouvre de nouvelles routes, et le fait arriver sans qu'il puisse se rendre compte de sa première impulsion. C'est ainsi peut-être que les logarithmes furent déduits pour la première fois de la progression des nombres, et le calcul différentiel de la méthode des tangentes. Ainsi, en parcourant les ouvrages des grands mathématiciens, on aperçoit une élégance, une adresse particulière dans l'ordre des déductions, ou le choix des transformations qui vont bien au-delà d'une connaissance profonde et d'une longue pratique des règles du calcul ${ }^{1}$. Ici, le génie n'est pas toujours asservi à la loi de continuité, soit qu'il combine des vérités abstraites, soit qu'il rapproche les faits de la nature extérieure, soit qu'il exécute des comparaisons imaginaires, il sait se créer à lui-même des archétypes; toujours le génie a ses analogies et une

d'être formé prépare l'exécution de celui qui va l'être, en s'associant avec lui. La même loi préside à nos perceptions directes et simples en apparence, comme à nos déductions artificielles les plus composées. Persistance des produits de l'activité dans le centre propre où ils se réfléchissent, qui fait les têtes fortes, capables de retenir et de combiner à la fois un grand nombre d'idées, c'est là peut-être ce qui forme la plus grande différence d'homme à homme. De bonnes habitudes premières fortifient cette heureuse disposition; on ne peut guère douter non plus que l'organisation naturelle du cerveau n'y entre pour beaucoup, mais il est essentiel d'observer que cette même disposition qui fait les penseurs profonds est presque toujours opposée aux habitudes de l'imagination ou d'une mémoire purement mécanique trop cultivée dans l'enfance.

Voyez en particulier l'algèbre de Newton et celle d'Euler. 
manière de les sentir qui lui est propre ; il n'imite pas, il crée ; il ne marche pas, il vole ; mais en rentrant dans l'ordre ordinaire et l'exercice réflectible de nos facultés, il est facile de voir que les traductions ou les substitutions successives de termes équivalents ou supposés tels, qui développent le raisonnement et en constituent la forme, n'en sont pas réellement le fond, ni la partie active et pour ainsi dire potentielle; il y a une traduction, pour ainsi dire spontanée, ou qui se règle sur les habitudes de la mémoire mécanique et il y en a une autre de choix et de réflexion. Un terme complexe peut être traduit de plusieurs manières ; quelle est celle qui est la plus appropriée au but intellectuel, et comment la reconnaître, sinon en comparant les termes donnés par une première traduction, ou les moyens intermédiaires entre eux, chacun à chacun, et avec le but général où l'on tend?

5. Si tous les termes élémentaires, idées ou signes, qui peuvent entrer dans une idée complexe donnée à analyser ou recomposer, étaient connus à l'avance ; si les diverses collections qui peuvent servir à former chacun de ces termes avaient été notées séparément et exprimées par autant de signes distinctifs analogues, s'il n'y avait à observer, dans les idées, que l'ordre de composition de leurs éléments, et que tous leurs caractères et leurs propriétés fussent exclusivement fixés par cet ordre invariable établi dans les signes, on pourrait dresser des tables et formules générales et symétriques, qui représenteraient aux sens toutes les manières différentes, dont une même idée peut se composer ou se résoudre. Ces formules s'approprieraient aisément à la mémoire ; il suffirait d'avoir exécuté une fois en détail et sur des exemples particuliers les compositions ou résolutions qu'elles indiquent, pour les appliquer avec assurance sans aucun retour sur leur premier fondement; bien plus, quand même ce fondement n'aurait jamais été aperçu, la pratique constante d'une méthode qui ne trompe point serait encore un titre de créance assez légitime. La mémoire des signes et des formes pourrait donc être isolée de la réflexion, et même de toute réminiscence des opérations exprimées en résultat, et cependant le but n'en serait pas moins atteint imperturbablement.

C'est alors que le raisonnement se trouverait réduit à une simple traduction du langage, et que la liaison des jugements pourrait n'être que celle des signes. Ce qui se passe dans les calculs de la routine, et dans la résolution des problèmes posés et énoncés dans la langue algébrique, où l'on n'a besoin de penser qu'en commençant l'opération et en arrangeant les données, les méthodes achevant tout le reste, ces exemples, dis-je, nous donnent l'idée de ce qu'est le raisonnement opéré par des traductions ou de simples transformations de signes.

Répétons-le dans un nouveau point de vue : les caractères spécifiques de nos idées mixtes ne dépendent pas seulement du nombre et de l'ordre de composition de leurs éléments, mais, avant tout, de la nature propre et du degré intensif de ces éléments divers. La plupart de ces idées se forment comme 
d'elles-mêmes, par un certain concours de circonstances, où chaque individu se trouve placé, etc. Il n'est donc pas question de connaître seulement ce qui se trouve renfermé, par suite de nos conventions, dans ces idées, et c'est presque toujours hors de leur sein, qu'il faut aller chercher les termes de rapport qui nous éclairent sur les détails de leur composition intrinsèque, lors même qu'il s'agit de descendre, par une réflexion concentrée, dans la constitution propre de l'une de ces idées ; une multitude d'éléments échappent, comme n'étant point naturellement dans la sphère de la mémoire ou n'y tenant par aucun signe déterminé qui leur assigne une place séparée. Les analyses qui se fondent sur de telles idées sont donc toujours très incomplètes, elles ne suivent presque jamais un ordre régulier, varient souvent dans le même esprit quant aux moyens, quant aux résultats ; c'est là aussi le domaine de l'imagination, c'est là que les liaisons fortuites et irrégulières des images commandent ou remplacent les liaisons réfléchies et fixes des jugements.

Quant aux idées, que nous avons le plus contribué à former nous-mêmes, telles que les idées générales ou abstraites des genres, des espèces, etc., leurs termes expriment les résumés d'une multitude d'expériences particulières ou d'observations que nous n'avons jamais eu l'occasion de faire, quoique nous en ayons les signes. Les déductions ou les comparaisons de ces termes ne pourront être alors ni très fécondes ni très régulières; mais admettons une institution exacte de ces idées complexes générales : elles n'ont pu d'abord se former qu'en prenant sur certains modèles des exemplaires particuliers, tel nombre de qualités ou de propriétés sensibles, qui, réunies sous un seul terme, auront constitué dans ce dernier le type de tous les individus, où les mêmes qualités se trouvent comprises. Or, ces qualités élémentaires peuvent bien être assignées en nombre mais non en intensité ${ }^{1}$, et quoique le terme général s'applique à toutes les espèces et tous les individus du genre ou de la classe, il est bien évident qu'il ne saurait les représenter tous également, sous le rapport même de la collection des propriétés qu'il exprime, ; qu'il conviendra plus particulièrement à ceux qui ont servi d'exemplaire dans la formation de l'idée générale et ne pourra s'appliquer aux autres que par supposition. C'est là ce qui induira souvent en erreur dans les comparaisons et déductions des idées de cet ordre où l'on affirme presque toujours l'identité de propriétés intrinsèques des diverses idées ou objets auxquels on peut appliquer le même terme générique conventionnellement institué : et il est en effet bien plus commode d'affirmer ou de supposer cette identité à la vue des signes, que de la vérifier dans les choses. Ici l'on peut reconnaître tout le danger de l'importance presque exclusive attachée aux classifications du langage, et du système qui fonde tout le raisonnement sur ses transformations.

C'est là ce qui trompe les mathématiciens eux-mêmes lorsqu'ils veulent introduire le calcul dans un système d'idées mixtes, dont les éléments quoique nombrables ont d'autres propriétés intensives qui échappent absolument à la langue précise du calcul. Cette illusion paraît bien surtout dans l'application; que de grands géomètres ont voulu faire de l'arithmétique et de l'algèbre à l'estimation des probabilités ! 
En effet, si l'on en excepte les idées vraiment archétypes dans l'ordre scientifique, comme dans celui de l'imagination, et celles de la quantité, dont l'homme peut être considéré comme l'auteur, toutes les autres ont un modèle ou un fondement, soit dans la nature extérieure, soit dans notre propre nature. C'est à ce modèle qu'il s'agit d'abord de conformer nos idées et puis les formules d'un langage qui doit les exprimer individuellement ou collectivement, suivant la distinction ou l'analyse des choses représentées, en partant des conventions ou des classifications mêmes de nos idées et de nos termes, pour fonder le raisonnement et un certain ordre régulier de déductions. Nous ne devons donc pas oublier qu'il y a avant tout des faits exemplaires individuels, différents ou analogues entre eux, et qui n'en restent pas moins tels, soit que nous les exprimions par des noms communs, ou par des noms propres, soit que nous échangions ou transformions leurs titres suivant nos hypothèses et nos méthodes, en renversant cet ordre et admettant pour toute règle nos propres définitions; à l'exemple des mathématiciens, nous pouvons opérer sur nos termes à peu près comme ils opèrent avec leurs signes ${ }^{1}$. Mais les suppositions du mathématicien ne le conduisent qu'à des résultats ou des vérités du même ordre hypothétique; la méthode qu'il emploie est telle d'ailleurs qu'elle redresse, dans la conclusion du raisonnement, les vices de l'énoncé, ou décèle les fausses égalités supposées, les erreurs commises dans le cours de l'opération; aussi peut-on s'endormir tranquillement sous la direction d'un tel guide. Nous ne sommes pas aussi heureux dans nos déductions logiques; admettant d'avance l'identité des idées ou des termes compris dans une même classe ou exprimés par les mêmes signes convenus, nous avançons toujours en prenant pour vrais (et absolument vrais) les résultats déduits de l'hypothèse fondamentale par une suite de transformations régulières, sans que rien puisse servir de preuve ou de correctif à l'hypothèse elle-même ; ainsi nous adoptons quelquefois, en principe, la conséquence nécessaire d'une fausse supposition première, ou nous affirmons l'identité des idées les plus diverses parce qu'en vertu des classifications ${ }^{2}$ d'un langage conventionnel nous sommes déterminés à leur appliquer les mêmes titres nominaux, tandis qu'au contraire

À la différence près des analogies sensibles, de la précision et de la clarté, que la nature de leur sujet a permis d'établir dans le matériel même de leur langue.

2 Lorsque plusieurs opérations précipitées ont concouru à former le titre d'un signe, il arrive ensuite que le signe lui-même sert de titre de créance à la régularité des opérations. Les exemples du calcul nous prouvent qu'après avoir déduit ainsi dans le principe la justesse d'une méthode générale de celle des opérations particulières qui s'y rapportent, nous finissons ensuite par appuyer au contraire toute notre confiance en la rectitude de ces opérations sur l'application de la méthode générale. C'est ce qui arrive toujours dans la répétition des mêmes procédés scientifiques; on forme des genres et des classes d'après les analogies plus ou moins exactes observées entre les choses ou les faits; puis on juge de l'analogie ou de l'identité même des choses particulières par l'application habituelle qu'on leur fait du même terme classifique et l'on se dispense désormais de tout examen ou comparaison de faits. Ce danger est bien grand surtout dans les idées qui n'ont point de modèle hors de nous. C'est là que la mémoire devrait toujours exercer une fonction réfléchie. 
la nature de la conclusion devrait nous rendre le principe ou le point de départ suspect dans le premier cas, et que la diversité réelle des idées renfermées sous un nom commun devrait nous faire scinder la classe dans le second cas, si nous avions une confiance moins aveugle dans nos méthodes, moins d'attachement peut-être pour des résultats éloignés, auxquels nous ne sommes parvenus que par de grands efforts de méditation, si nous avions posé une ligne de démarcation plus fixe entre le domaine de la vérité hypothétique et celui de la vérité absolue, enfin et surtout s'il existait un criterium auquel nous puissions rapporter avec assurance les idées qui, n'ayant point de modèle au dehors, ont pourtant dans l'observation de nous-mêmes une règle que l'on ne peut mettre à l'écart et qu'il dépendait peut-être de nous de rendre moins incertaine.

\section{V. - De l'évidence métaphysique. Obstacles qui nous empêchent d'en avoir une parfaite dans la science de nos facultés. Conclusion.}

$\underline{\text { Retour à la table des matières }}$

Ce critère, cette lumière de l'évidence que nous cherchons, ne peut nous venir entièrement du dehors, nous ne le trouverons point uniquement dans les rapports de nos signes à nos idées, ni dans une identité conventionnelle et bien souvent illusoire. La source de l'évidence doit être plus réelle et plus près de nous; il y en a une métaphysique comme il y en a une mathématique, toutes deux également fondées sur le fait primitif d'existence.

Notion objective du terme de l'effort et toutes les idées qui dérivent de la même origine sans aucun autre alliage : évidence mathématique.

Conception réflective du sujet moi de l'effort et tous les actes de nature identique à celui qui fonda le premier sentiment individuel d'existence : évidence métaphysique, inséparable de la première.

Le mathématicien placé à la source conçoit et se représente; il a des signes permanents inséparables des idées comme de l'objet qui sert en même temps de modèle et d'appui aux unes et aux autres. Ici seulement l'évidence réelle et logique se confondent ou sont indivisiblement unies.

Le métaphysicien placé à la même source conçoit et réfléchit il s'arrête aux limites du monde extérieur; le terme objectif de l'effort n'est pas pour lui la base fixe sur laquelle reposent ses idées, mais un point d'appui d'où part la 
pensée pour se réfléchir sur elle-même; ses signes sont les actes mêmes qui lui révèlent sa puissance constitutive, ses vouloirs, son existence; mais de tels signes sont transitoires dans l'ordre successif, et n'ont point dans leur nature cet attribut essentiel de permanence qui n'appartient qu'à l'ordre des coexistants.

Les conceptions du métaphysicien échappant aux signes extérieurs, l'évidence ne peut y être qu'immédiate pour le sujet même et incommunicable par des moyens artificiels. Au contraire l'évidence mathématique empruntée du dehors y revient et n'existe qu'en se communiquant par des signes clairs et durables.

Mais en avançant et s'éloignant de la source, la science mathématique pure peut admettre plusieurs éléments hétérogènes; en s'associant à la science des qualités physiques passagères ou variables de l'objet extérieur, elle perd une partie de la certitude qu'elle communique; à mesure que son objet se complique, ses représentations deviennent plus incertaines et ses signes souvent trompeurs par le caractère même de simplicité et de fixité absolue qui fait leur essence.

Le véritable objet métaphysique s'enveloppe aussi en se combinant ; tous les caractères de l'évidence intérieure s'effacent à mesure qu'il admet une multitude d'éléments hétérogènes qui, émanés d'une autre source, l'altèrent par leur mélange ; ainsi il peut perdre toujours pour s'éclairer lui-même la portion de lumière qu'il leur communique.

Alors les sages, fidèles au précepte de l'oracle ${ }^{1}$, ne trouvent plus qu'obscurité en eux-mêmes; tout conduit à aller au dehors rallumer un flambeau éteint; frappés de l'éclat d'une lumière empruntée, c'est à elle que leurs yeux s'attachent, c'est elle seule qu'ils reconnaissent comme propre et naturelle ; c'est dans les objets sensibles de leur conception, en s'élevant jusqu'aux cieux ou descendant dans les abîmes, qu'ils contemplent la pensée : c'est ensuite dans les signes ou les résultats de leurs propres conventions, qu'ils saisissent ses formes extérieures. Embrassant alors une nouvelle sorte d'évidence logique, ils s'y tiennent comme à la seule qu'il soit donné à l'homme de connaître, et qu'il lui soit permis de rechercher. L'évidence métaphysique ne peut plus subsister pour nous dans sa pureté. La raison n'en est pas seulement dans le défaut des signes, qui tient lui-même au caractère des faits, souvent incommunicables par le langage, lors même qu'ils sont immédiatement déterminés pour la réflexion mais c'est surtout que l'observation de ces faits plus incomplète, sujette à des difficultés extrêmes, trouve des bornes naturelles dans l'hétérogénéité même du sujet à qui elle tend à s'appliquer.

\footnotetext{
Nosce te ipsum.
} 
En faisant abstraction de cette vue même toute extérieure qui nous emporte si loin de nous, et nous cache ces formes intimes qu'elle confond avec l'objet, ou dont elle l'habille, nous supposant réduits à ce qui peut faire le sujet et la matière d'une observation intérieure, à la réflexion de nos actes et au sentiment immédiat de nos propres impressions ; nous trouvons là, encore, deux sortes d'éléments en opposition et presque toujours en lutte ; et pourtant c'est de leur combinaison que résulte l'homme tout entier; ce n'est que dans l'exercice simultané de deux forces et dans l'application de l'une à l'autre et à elle-même, que l'être intelligent et sentant peut s'étudier et se connaître dans l'humanité complète (duplex in humanitate).

L'aperception ou le principe de la connaissance n'est point dans les affections d'une vie toute intérieure, et nous avons vu pourquoi ou comment, quand ces affections dominent, elles absorbent tout ce qui n'est pas elles. Cependant, l'homme ne peut se connaître sous le rapport moral en particulier ${ }^{1}$, sans tenir registre, pour ainsi dire, de ses affections et de leurs produits immédiats, ce qui suppose la possibilité de leur appliquer une sorte de tact intérieur, dont les hommes, distraits par tant d'impressions vides du dehors, sont peu disposés à faire usage ; mais de plus, cette sorte de tact affectif se trouve liée à une certaine disposition du tempérament organique, la même qui avivant les impressions ou en multipliant les causes, les rend aussi souvent tumultueuses et confuses dans le sujet et y annule toute capacité d'observation ; il suffit encore de vouloir appliquer à ces produits d'une sensibilité spontanée, bien indépendante dans son principe, un autre sens plus réfléchi qui ne leur est point directement approprié, pour qu'elles fuient et se dénaturent : c'est Eurydice dont le souffle de vie s'évanouit par un simple regard.

Ainsi donc, l'analyse de ce premier ordre de facultés affectives considérées dans leur rapport avec la pensée sur qui elles exercent un ascendant si puissant, si continuel et si inaperçu, trouve des bornes nécessaires et des obstacles insurmontables dans la nature même de l'organisation, puisqu'elle exigerait une réunion de circonstances presque incompatibles : assez de mobilité dans les impressions pour que l'individu puisse les comparer ou se comparer à luimême (sous ce rapport) dans divers états ; assez de force et de persistance pour pouvoir les distinguer, s'en rendre compte, et puis une sensation telle que les affaires ou les choses du monde extérieur eussent peu de prise sur nous ${ }^{2}$, et puis encore les signes qui manquent, les termes et les classifications du langage, inapplicables à tels objets, etc. C'est ici que la science physiologique,

La morale philosophique peut être considérée comme la science de nos facultés affectives et intellectuelles prises dans leur influence nécessaire et réciproque. Elle se fonde surtout sur les deux sortes d'observations intérieures dont nous parlons, et qui sont si sujettes à s'obscurcir ou à s'effacer l'une par l'autre. La théorie des sentiments moraux serait à celle des facultés intellectuelles pures (si nous pouvions considérer ainsi ces dernières), ce qu'est la science des mathématiques pures à une étude physico-mathématique.

Quid tyridatem terreal unice securus. 
qui déduit l'ensemble des signes physiques des faits de cet ordre, peut fournir quelque lumière à l'observateur moral, pourvu que celui-ci ne confonde pas les signes avec les choses, comme on s'y trouve naturellement porté dès qu'on se place dans le point de vue objectif.

La réflexion (ou l'observation intérieure considérée dans son champ propre) ne peut s'appliquer directement qu'aux actes ou aux produits de la volonté qui deviennent, par là-même, ceux de l'intelligence. C'est là en effet que le moi est constitué directement observateur de ce qu'il est et de ce qu'il fait. Mais d'abord le sujet n'est point ainsi exclusivement constitué in abstracto, dans son aperception interne immédiate ; il y a des conditions instrumentales, pour ainsi dire, auxquelles il doit se rattacher dans le fait primitif de conscience.

Or, cette connaissance tient encore à une sorte de sentiment immédiat et exige des considérations d'un autre ordre ${ }^{1}$. Mais quand même nous devrions nous réduire à ces sortes de formes ou de produits intellectuels qui sont du domaine exclusif de la réflexion, combien de difficultés et d'obstacles encore à cette étude ! Nous avons assez vu avec quelle promptitude et quelle nécessité l'aperception intérieure se transforme ou s'absorbe dans les sensations et les intuitions spontanées ou les résultats de nos actes mêmes. Les signes, dans leur institution secondaire sont à peine une barrière suffisante pour retenir ou préserver l'aperception de cet enchaînement de l'habitude. Ajoutez les obstacles naissant d'un langage qui ne peut exprimer un seul point de vue pris en nousmêmes, sans une métaphore qui nous rappelle au dehors; ajoutez encore l'influence essentielle des affections immédiates du tempérament, dont l'exercice de la réflexion ne peut jamais entièrement s'isoler, et qui rend chaque observateur si différent de tout autre, si différent de lui-même en divers temps, que les faits évidents pour celui-ci paraissent souvent des illusions ou des chimères à celui-là, sans qu'ils aient presque aucun moyen de s'entendre ; et que la vérité de sentiment intérieur, qui nous frappe vivement à cette heure, s'obscurcira peut-être dans l'heure suivante.

Convenons-en donc : quoiqu'il existe une source réelle et pure de l'évidence métaphysique, il résulte même de notre nature mixte que nous ne pourrions avoir maintenant un criterium bien assuré, une mesure invariable ni des signes proprement expressifs d'une telle évidence ; mais les obstacles à la science (et ceci est bien remarquable) ces obstacles, dis-je, font partie de la science même. Je dois donc me croire bien loin d'avoir établi quelque science positive, et je me féliciterais bien, si j'avais fait une petite partie de son introduction.

Je trouve en moi-même et j'établis dans l'homme, trois sortes de facultés non séparées, mais aussi réellement distinctes entre elles, que le sont les organes particuliers des sens, sur qui elles se fondent : facultés :

Nouveau recours nécessaire à la physiologie. 
1. Affectives, qui constituent comme la base et la matière première de son être.

2. Représentatives, qui l'informent de ce qui est au dehors et fixent les limites.

3. Aperceptives ou réfléchies, par lesquelles il prend connaissance et de ses affections et de ses idées, et de lui-même comme sujet pensant et sentant.

Si l'on voulait encore réduire sous un seul terme tel que sensation, tout ce que l'homme sent et perçoit au dedans comme au dehors, du moins faudrait-il convenir, que ce par quoi il est lui persistant et le même quand tout varie, ce par quoi il est capable d'affirmer en lui-même certaines propriétés ou attributs des existences étrangères, comme de la sienne propre, de reconnaître enfin ce principe même que tout se réduit à sentir, etc., n'est pas la sensation toute seule ; il faut un mot propre individuel et non commun, pour exprimer la puissance une et identique de cet acte affirmatif, de tous rapports d'inhérence des modifications au moi, d'impressions sensibles aux organes, de qualités perceptibles aux objets; ces deux derniers rapports ne pourront non plus être confondus sous la même expression pas plus qu'ils ne le sont dans le sens intérieur.

Ce n'est assurément rien dire de nouveau que de distinguer ces trois facultés, ou si l'on veut ces trois propriétés de notre être, par lesquelles nous sommes capables d'éprouver simplement le plaisir ou la douleur, de nous représenter quelque chose hors de nous, et d'apercevoir plus ou moins confusément nos actes ou leurs résultats, en conservant le sentiment individuel de notre existence et demeurant le même moi. Je crois bien que tout le monde sent cela à peu près de la même manière, quoique chacun puisse l'exprimer diversement à la sienne ; mais ce qui peut être, je crois, considéré comme assez nouveau, c'est d'avoir entrepris de rapporter chacune de ces facultés (et la dernière surtout) à une source ou à une condition particulière prise dans l'organisation elle-même, d'avoir cherché à les reconnaître individuellement dans divers produits où elles se combinent intimement, quoique d'une manière inégale, d'avoir fait en quelque sorte la part de chacune dans les composés de l'ordre sensible comme de l'ordre intellectuel, d'avoir montré enfin le parallélisme de ces deux ordres qui ont toujours été, si je ne me trompe, ou trop séparés, ou trop confondus. C'est là ce que j'appelle une décomposition sinon de la faculté de penser une et individuelle, du moins des facultés de l'homme.

Là est aussi pour moi et dans mon sens intérieur une évidence métaphysique, non celle qui se conserverait toujours également pure dans les actes de l'intelligence ainsi reconnus, énumérés avec une exactitude parfaite et rattachés à des signes clairs et déterminés, qui emportent dans l'esprit de tous 
un sens univoque (je ne prétends pas m'être dirigé aussi heureusement d'après une telle évidence); mais celle qui consiste à rapporter, d'après des signes certains pris dans la nature des choses, chaque ordre de produits à la source où il prend naissance, celle qui applique le sentiment immédiat aux affections de la sensibilité intérieure, tel sens externe à la représentation, tel autre à la connaissance objective ou la perception, tel mode enfin d'exercice de tous ceux qui obéissent, d'après certaines conditions, à la même volonté, à la réflexion, simple et redoublée des actes et de leurs résultats.

Du concours de ces trois ordres ${ }^{1}$ de faits distincts et hétérogènes dans leur source, je vois ressortir le phénomène mixte de l'humanité ; mais je trouve aussi dans ce concours nécessaire la cause réelle qui nous empêche de reconnaître ou d'obtenir les véritables éléments dans chaque ordre séparé, et de là suit cette dernière conséquence : si la question sur les facultés élémentaires n'est pas résolue aussi exactement qu'il eût été possible par l'application de la méthode que j'y ai employée, cette méthode même prouve qu'il est impossible de la résoudre complètement dans son étendue, et de manière qu'il n'y ait plus lieu à y revenir ; cette démonstration, tirée de la nature du sujet, me semblerait équivaloir à une solution réelle.

C'est avec une sorte de confiance que j'offre à des philosophes, qui s'intéressent aux progrès de la science de l'homme, la méthode dont je ne suis servi pour éclaircir une question fondamentale de cette science. Je crois fermement que c'est la seule appropriée à des questions de ce genre ; je crois fermement que pour connaître l'homme il ne suffit pas d'observer le jeu et les fonctions de ses organes ou leurs résultats immédiats, pas plus que de se concentrer dans l'enceinte de sa propre pensée, en faisant abstraction de ces sentiments et de ce jeu conditionnel nécessaire, car ainsi on est conduit à bien des illusions; ou l'on prend les signes pour les choses mêmes, ou l'on établit la science avant l'existence même, en la fondant sur des principes purs et synthétiques a priori.

Il ne faut pas voir tout exclusivement en dehors ni tout en dedans, mais à la fois en dehors, dans l'organisation intime, et en dedans de la pensée, et cela justement parce qu'il y a trois sortes de facultés à connaître et qu'il faut une méthode qui puisse les embrasser toutes.

Je demande au surplus (et j'aurais peut-être le droit d'exiger) qu'on ne juge pas cette méthode que je recommande, par l'application sans doute très

On pourrait dire qu'il s'agissait uniquement dans la question proposée des facultés intellectuelles et non de l'ordre des facultés affectives et morales. Je crois avoir bien fait voir l'impossibilité de séparer les trois ordres, lorsqu'on veut commencer par le commencement, marcher droit et connaitre le tronc avant de s'attacher à quelques branches isolées. L'impossibilité démontrée de scinder ainsi des questions fondamentales, telle que la proposée, est même un des principaux résultats de ce travail. 
imparfaite que l'on en trouve dans ce long mémoire. Tout m'a manqué à la fois pour exécuter un plan conçu sans avoir assez consulté mes forces, et sans prévoir des circonstances bien funestes qui en ont traversé l'exécution.

Tel qu'il est, je le livre à la méditation des sages qui sauront bien séparer le bon suc et rejeter le caput mortuum.

\section{Résumé général}

\section{Projet d'une analyse de décomposition des facultés humaines, d'une division des modes de la sensibilité et des opérations élémentaires de l'intelligence.}

\section{$\underline{\text { Retour à la table des matières }}$}

La notion, exprimée dans le terme faculté, s'individualise comme idée simple d'une cause ou force productive, moi, des mouvements et actes qui s'y réfléchissent; ou se généralise comme idée abstraite d'une capacité ou possibilité de recevoir diverses impressions dans les différentes parties sensibles du corps.

On peut énumérer les actes réflectibles de la même puissance individuelle, ou classer les modes passifs, suivant certaines analogies relatives au point de vue particulier sous lequel on les considère, comme d'affecter un siège organique déterminé, d'être le résultat du jeu et des fonctions de ce siège, de la rapporter à telle cause extérieure capable de les produire, etc. De là trois méthodes d'analyse :

1. Méthode réfléchie ou métaphysique qui a pour objet l'énumération des actes de l'intelligence et peut se proposer aussi une décomposition réelle des produits mixtes, où certains modes potentiels réflectibles (sous le nom de formes), se trouvent unis à tels autres modes d'affectibilité (sous le nom de matière).

2. Méthode analogique qui, sans avoir égard au composé, se propose de distinguer les caractères d'une même sensation, et en forme les titres de divers genres ou espèces de modes passifs, sous le nom de faculté. 
3. Méthode physiologique qui, considérant les facultés humaines comme fonctions des organes ou résultats de ces fonctions, se propose de classer ou d'analyser les unes comme les autres dans le rapport exclusif aux instruments divers et au jeu des organes, d'où résultent les différentes espèces de sensations.

La première méthode s'attache principalement à ce qui forme la constitution intérieure de l'être pensant : les deux autres tracent plutôt en dehors les limites de l'être qui sent et se représente. En se plaçant tour à tour dans ces trois points de vue, pour embrasser l'ensemble des phénomènes de la sensibilité et de l'intelligence, on doit reconnaître deux ordres de facultés, l'un actif, l'autre passif.

\section{Ordre passif (simple)}

Retour à la table des matières

Facultés passives: capacité affective générale. Résumé de tous les modes de ce genre dont l'organisation vivante est susceptible dans son ensemble et chacune de ses parties. Première: dans l'ordre de l'existence, quoiqu'elle ne le soit pas dans l'ordre de dérivation des facultés qui constituent l'être intelligent ou pensant.

La faculté générale réceptive de toutes les impressions quelconques immédiates peut s'exprimer par le terme affectibilité, propre à la combinaison organisée vivante. Ainsi considérée dans son état primitif et pur, elle ne peut être prise comme origine de dérivation d'aucune des facultés intellectuelles. Éléments sensibles, doués par leur nature de quelques impressions obscures, qui s'avivent réciproquement dans leur réunion ou leur coordination en système. Monades de Leibnitz, perceptions obscures. Molécules organiques de Buffon, sensation matérielle, espèce de sentiment absolu et fondamental inséparable de la vie organique. Fonctions de cette vie, affectible immédiatement, dans les résultats généraux et particuliers.

a) Modes généraux du sentiment absolu. Déterminations instinctives premières. Instinct de nutrition et de conservation. Affinités organiques ou animales. Affections du tempérament propres à l'espèce ou à l'individu. Sentiment radical de force ou de faiblesse ; courage ou timidité ; bien ou mal, aise, inquiétude, besoin, etc., affections incessamment variables en degrés par 
toutes les causes externes ou internes, dans l'état de santé, dans la maladie, dans les âges, les saisons, les climats, etc.

b) Résultats de fonctions particulières aux organes impressionnables au dedans ou au dehors. Modes divers du plaisir ou de la douleur, subdivisibles en autant d'espèces qu'il y a de parties de fibres ou d'éléments affectibles, doués chacun de leur impressionnabilité propre et spécifique. Point de fondement à une classification régulière ; impressions immédiates toutes semblables à celles des organes internes, toujours générales : ne se circonscrivent point d'ellesmêmes dans un siège ; se confondent dans leur ton naturel le plus bas, comme dans ce degré élevé qui les constitue animales.

c) Tact passif de toutes les parties extérieures du corps. Odeurs et saveurs liées par une sympathie étroite et naturelle à l'instinct de nutrition et de conservation. Impressions immédiates de fluides lumineux et sonores sur les organes de la vue et de l'ouïe. Affections générales produites par les soins dans le système sensitif, sans aucune audition. Pouvoir, qu'a le physique, ou le matériel même, de tels sons, pour exciter directement certaines passions, quelquefois guérir certaines maladies, etc.

d) Impressions bien particulières (et non affectibles) du fluide lumineux sur la rétine. Propriété vibratoire du sens externe ou interne qui les reçoit. Faculté naturelle de représentation inhérente à ce sens, indépendamment de tout acte de perception ou de connaissance (par exemple instinct des animaux naissants qui vont juste atteindre l'objet approprié aux besoins de nutrition). Production ou conception spontanée des images dans une des divisions de l'organe cérébral, soit directement, soit en vertu de l'irradiation sympathique de certains organes internes. Fantômes légers ou persistants dans les songes, le délire, l'aliénation mentale, divers états vaporeux, etc. Imagination passive ou résultat immédiat de l'impressionnabilité cérébrale propre. Images qui coïncident avec les besoins de l'animal et les modes variables de son affectibilité intérieure, etc., toutes facultés passives qui se distinguent ou se caractérisent par des signes pris dans le physique même (ou ensuite dans une autre sorte d'observation liée au sentiment immédiat). Ces facultés peuvent toujours être considérées en dehors de l'intelligence; elle n'y est point originairement constituée puisque la perception et la pensée peuvent s'en 
trouver complètement exclues ${ }^{1}$. Objet physiologique qui ne forme pas toute la science de l'homme moral et intellectuel, mais dont celle-ci ne peut se passer ${ }^{2}$.

1 Remarquez aussi que les facultés qui se rapportent à un tel état sensitif, bien loin d'être les premières qui se présentent dans une analyse réfléchie de nos facultés, sont au contraire tout à fait écartées et méconnues, lorsqu'on prend exclusivement son point de départ dans une méthode de réflexion, et qu'on veut se diriger d'après elle. Voyez Descartes, Leibnitz et leur école ; ils intellectualisent les affections directement simples de la sensibilité, ou n'en parlent pas.

2 Voyez dans tout ceci le chapitre I de la seconde partie du Mémoire et surtout les belles analyses contenues dans le livre des Rapports du physique et du moral de l'homme. 


\section{Ordre actif (simple)}

$\underline{\text { Retour à la table des matières }}$

Faculté ou puissance individuelle unique de mouvement et d'action. Mode actif fondamental élémentaire par rapport à tous ceux de cet ordre.
La faculté ou force une, qui produit avec conscience ou sentiment d'elle-même tous les actes ou modes intellectuels proprement dits, est cette même puissance qui, sous le titre individuel (propre et non commun), est capable de créer l'effort, mode relatif fondamental.

a) Origine réelle (ou dérivative) de tous les modes du même ordre les plus éloignés de la source commune. Volonté bien inséparable de l'entendement qui n'est constitué qu'en elle ou par les résultats premiers de son exercice.

b) Condition première du déploiement de l'effort, centre direct de motilité volontaire (point de vue physiologique). Homogénéité et constance des instruments organiques, sur qui et par qui se déploie la force motrice identique, comparées aux variations que l'on peut observer dans les organes sensitifs, doués chacun d'une impressionnabilité spécifique différente. Effort variable seulement par le degré, condition nécessaire d'un sentiment fondamental aperçu. Aperception de moi dans l'exercice de la motilité volontaire. Identité ou réminiscence personnelle. Fondement de la mémoire dans la continuité ou la répétition libre des mêmes actes. Forme du temps. Sentiment et idée primitive et simple de causalité. Modèle premier de toutes les idées de force ou de puissance, transportées par la suite dans la nature extérieure. Source commune de toutes les idées simples réfléchies d'unité, d'identité, etc. Objet métaphysique. Énumération et distinction des actes, modes ou opérations individuelles exprimés par différents signes. Méthode appropriée à cet objet (point de classification idéologique). (Voyez chapitre II, $1^{\text {re }}$ section.) 


\section{Ordre passif (composé)}

Première classe de composés sensibles par association simple ou par agrégation ${ }^{1}$ des deux forces élémentaires, l'affectibilité prédominant ou fournissant toute la mati ère de la sensation.

$\underline{\text { Retour à la table des matières }}$

Sensibilité, faculté de sentir ou d'éprouver des modifications quelconques, le mot n'étant pas identifié avec ces modifications, puisque sa puissance constitutive ne contribue pas à les produire.

A. Premières sensations composées.
Le mode relatif fondamental d'effort s'associe ou s'agrège simplement avec diverses impressions affectives qui passent, se succèdent ou persistent, sans dépendre en aucune manière de la puissance moi qui les perçoit, et peut être dite les sentir, comme produits d'une autre force, mais ne peut les devenir ou s'identifier avec elles, sans cesser de s'apercevoir, d'exister pour elle-même. Cette puissance transporte, pour ainsi dire, dans l'état affectif, les formes invariables qui lui sont propres, constitue dans l'existence absolue une relation première, une succession, une durée mesurée, un temps, toutes formes qui ne sont point inhérentes à l'affectibilité simple et peuvent en être séparées. Caractère fondamental qui distingue ce premier ordre de composés (ou la sensation), de l'affection pure organique, premier point de départ d'une analyse de décomposition. Rencontre et concours de la physiologie et de la métaphysique qui considèrent chacune leur élément dans le même composé. Séparation des deux points de vue, à partir de ce terme, soit pour s'élever dans l'ordre intellectuel, soit pour descendre dans l'ordre des phénomènes purement organiques.

\footnotetext{
Les métaux ne se combinent point directement avec l'oxygène pour se transformer en acides, mais doivent d'abord passer par un premier état d'oxydation, avant de parvenir à cette combinaison intime qui en fait de véritables acides métalliques. Ainsi (et autant qu'il est permis de comparer des modes intérieurs à des choses qui se représentent), l'affection pure ne se transforme point d'elle-même dans les formes individuelles et actives d'attention, de comparaison, de mémoire, mais seulement après avoir passé à l'état de sensation, où elle est déjà unie à une première forme simple de personnalité. Cette image, infidèle sans doute, se rapproche assez pourtant d'un point de vue intérieur, que je voudrais pouvoir exprimer, comme je le conçois.
} 
B. Sensations générales qui ne sont rapportées à aucun siège déterminé.
Sensations proprement dites de malaise, d'inquiétude, de besoin, etc. Passions simples et non encore composées par l'imagination. Ensemble de tous ces modes généraux du plaisir ou de la douleur que l'on a compris sous le terme générique volonté. Résultat immédiat de l'affectibilité intérieure unie à un sentiment de personnalité plus ou moins confus, et qui peut souvent y être absorbé.

Seconde classe de composés sensibles.

A. Sensations doublement composées.

L'exercice du toucher donne un objet ou une cause extérieure à diverses modifications de la sensibilité. Le jugement d'existence étrangère, directement fondé sur cet exercice, s'associe avec les premières sensations déjà composées et les surcompose. Distinction essentielle à établir entre les modes perçus et non affectifs qui se rapportent à l'objet comme qualités, et les modes sentis qui se réfèrent simplement à la cause étrangère. (Voyez chapitre V.) Le jugement d'extériorité collélatéralement uni avec les affections simples, n'y est pas plus essentiellement renfermé que la personnalité même. Ces affections n'ont qu'.... pour absorber tout jugement de cette espèce ; et quand on pourrait dire, dans un autre point de vue, que le rapport est éminemment senti, c'est alors même qu'il n'y a plus de jugement. De même, quand la sensation dite animale est au plus haut degré, l'attention est nulle comme puissance. C'est quand l'ébranlement organique persiste avec le plus de force, qu'il n'y a pas de souvenir. C'est quand le besoin est extrême, qu'il n'y a pas de vouloir, etc.

Troisième classe de composés sensibles.

B. Désirs ou besoins et passions simples, composés avec le jugement.
Dans la connaissance objective, le besoin étant accompagné du jugement d'existence ou de la représentation de l'objet propre à faire cesser le malaise, ce besoin simple, dis-je, devient la matière d'un désir proprement dit, composé de troisième classe: sensation, jugement, souvenir o u imagination intellectuelle jointe 


C. Sentiments et pas-
sions mixtes ou
composées d'actes
intellectuels.

Quoique nos affections de toute espèce soient hors des limites de la volonté, il est pourtant des sentiments qui naissent à la suite des actes intellectuels, et s'allient avec la réflexion et un exercice régulier de la pensée dans l'emploi des signes institués. Ces sentiments ou passions mixtes doivent former une classe à part ; il en sera parlé ciaprès ${ }^{(1)}$.

(1) Nos passions ne peuvent pas être directement excitées ni ôtées par l'action de la volonté ; mais elles peuvent l'être indirectement par la représentation des choses qui ont coutume d'être jointes avec les passions que nous voulons avoir, et qui sont contraires à celles que nous voulons rejeter. Ainsi pour exciter en soi la passion hardiesse et ôter la peur, il ne suffit pas d'en avoir la volonté mais il faut s'appliquer à ne considérer les raisons (DESCARTE, Traité des passions de l'âme). La volonté produisant donc ainsi immédiatement les idées et immédiatement les passions associées à ces idées, il peut y avoir perception ou aperception dans ces émotions de l'âme qui sont purement affectives, quand elles sont spontanées. 


\section{ORDRE ACTIF (composé)}

Première classe de composés perceptifs, par combinaison intime des deux premiers éléments, la volonté concourant avec une force extérieure à former la perception.

$\underline{\text { Retour à la table des matières }}$

A. Perceptibilité modale ou objective, faculté de percevoir des impressions, non essentiellement affectives, telles que les couleurs et les sons. Modes perçus par suite d'une action de la volonté plus ou moins expresse, toujours nécessaire pour les produire. Odoration, saporation, mais surtout vision et auscultation active.

\section{B. Faculté d'attention.} Attentivité.
Dans l'exercice de cette faculté (propre à la vue et à l'ouïe), le sens n'est pas absolument réduit à atteindre passivement les impressions de l'objet; activé par la volonté, il va au-devant d'eux, s'y applique et en retire des informations plus exactes et plus détaillées : la force motrice peut y prendre l'initiative, sans conserver la prédominance sur la force extérieure. L'exercice de cette faculté se fonde essentiellement sur le déploiement de l'effort : il emporte donc avec lui les formes de personnalité et de jugement qui ne peuvent y être absorbées, comme dans la sensation, dont le mobile est tout en dehors du vouloir. Acte perçu seulement en résultat. Point d'aperception relative à cet acte même. Jugement composé où tout se rapporte à l'objet. Idée complète mais indécomposable dans ses éléments qui ne peuvent être conçus l'un hors de l'autre (le mode, hors du sentiment personnel) quoiqu'ils puissent être distingués dans leur prédominance alternative.

J'appelle ainsi (attention) la détermination plus expresse du vouloir qui active le résultat perceptif et lui donne un caractère de distinction, de vivacité prédominante, qui affaiblit et quelquefois annule pour la conscience toute impression simultanée.

Il paraîtrait que l'attention n'est qu'un degré particulier de la perception, et qu'il est inutile de les distinguer comme deux facultés propres (et sui generis). Cette objection est fondée, lorsque l'on confond la sensation avec la perception, mais en tant qu'il y a dans cette dernière deux éléments combinés et deux forces concourant à la produire, en tant........ représenté pas plus dans le souvenir que dans le sens. Ce n'est donc point son être propre que l'individu reconnaît dans l'image reproduite ; mais 
E. Imagination intellectuelle. Image accompagnée de réminiscence sans qu'il y ait possibilité de rappel direct. l'identité personnelle inhérente à l'aperception du vouloir ou de l'acte répété, se transforme toute dans l'identité objective ou modale. Dès que cette aperception est entièrement confondue dans le résultat sensible du même acte, l'antécédent disparaît ainsi dans le conséquent; le représentant se cache, et le représenté seul se montre; mais quoique l'activité ne subsiste qu'en résultat ou dans l'image c'est toujours elle qui fonda cette sorte de réminiscence étrangère et comme impersonnelle; et l'image n'est pas plus le souvenir, que l'affection n'est la sensation, ou que celle-ci n'est la perception.

Il importe de ne pas confondre ce mode de production des images (auxquelles l'exercice de l'attention a imprimé le cachet de la réminiscence), avec l'imagination purement passive qui exclut toute forme de personnalité, d'intelligence, et n'est que l'exercice même de l'affectibilité cérébrale. Il y a là un progrès bien notable de cette dernière espèce d'imagination à celle qui est accompagnée de réminiscence, comme celle-ci à la mémoire. (Voyez chapitre II.)

Seconde classe de composés aperceptifs. La volonté prenant l'initiative et conservant la prédominance sur la force extérieure.

A. Aperception objective. Faculté d'apercevoir simultanément dans le même mode (effort) l'action que la volonté détermine et son résultat permanent au-dehors.

Toucher actif. Attention et réflexion dans l'exercice de ce sens spécial.

B. Jugement simple. Origine d'une double observation.

C. Jugement composé. Comparaison des idées.

D. Mémoire des formes
L'aperception est ici dans l'acte même déterminé hors du contact, la perception objective dans le résultat qui se rapporte au dehors où la volonté va le chercher. Rencontre directe de deux forces vivantes ou d'une force vivante moi, et d'une force d'inertie morte, qui attend l'action sans la prévenir. Idée complète. Source d'une double évidence où le métaphysicien et le géomètre puisent également les idées simples, réfléchies pour l'un, et abstraites réfléchies pour l'autre; d'unité, de force séparée de l'étendue, de substance identique permanente, etc.

Fondement d'une synthèse réfléchie dans la conception du rapport simple de la force agissante, à la résistance. Fondement d'une analyse imaginative dans la conception du rapport composé pris pour origine. Ici, juger c'est sentir; là le jugement est hors de la sensation. L'analyse complète des fonctions du toucher fixe le vrai point de départ de l'idéologie. (Voyez chapitre V.) 
de cette faculté développée ensuite dans un autre ordre d'association.
Passage de l'imagination intellectuelle à la mémoire. Rappel complet des idées de forme. Réminiscence personnelle et objective assurée dans le déploiement constant de la même activité, etc.

Troisième classe de composés aperceptifs.

La volonté donnant et fournissant toute la matière du produit aperçu.

A. Aperception réflective. Faculté d'apercevoir ... volontaire dans résultat ...... sons qui la reçoit du dedans en dehors, exercice unique de l'oüe.

Type et fondement de la réflexion proprement dite.

B. Origine et fondement de l'institution des signes.

C. Mémoire et rappel complet des sons oraux. Double réminiscence.
La détermination vocale est aperçue à la fois et instantanément en elle-même et dans le résultat ou l'impression auditive qui acquiert ainsi, à l'exclusion de toute idée sensible, cette propriété de redoublement intérieur caractéristique de la réflexion. C'est là qu'est la source et le mobile de cette faculté, séparée par Locke de la sensation, et identifiée ensuite par Condillac avec toute sensation en général.

Le mouvement vocal ou oral et la perception auditive interne se trouvent compris indivisiblement dans un vouloir unique et constituent une seule action ou idée complète. loi encore le simple s'enveloppe et le composé seul se réfléchit. C'est ce composé qui devient signe et qui réunit bien toutes les conditions d'un véritable signe intellectuel.

Les mouvements vocaux sont aux impressions directes de l'ouïe ce que les actes propres du toucher sont aux simples sensations de tact, ou encore aux représentations passives des couleurs. Tout ce qui se trouve immédiatement lié à ces deux sortes de mouvements dans l'exercice respectif de l'ouïe et du toucher, est par là même dans la mémoire, ou docile au rappel ; le reste en est séparé ou ne subsiste que dans l'imagination spontanée. Ainsi comme la mémoire des formes et des figures peut être séparée de la représentation des nuances de couleur, et l'est toujours du souvenir des sensations de tact (telles que le chaud, le froid, etc., qui ne se reproduisent point), ainsi la mémoire des sons articulés subsiste nettement, tandis que les qualités du son qui s'adressent seulement à l'ouïe, échappent au rappel. Il n'y a d'idée et de véritable mémoire que des 
mouvements ou actes, et par suite, des choses imitables par ces mouvements, que la volonté répète. Or, nulle sensation ou chose simplement sentie n'est imitable, et les deux seuls sens imitateurs sont la main et la voix ; ce sont eux seuls (ou ...)

$\underline{\text { Retour à la table des matières }}$ 